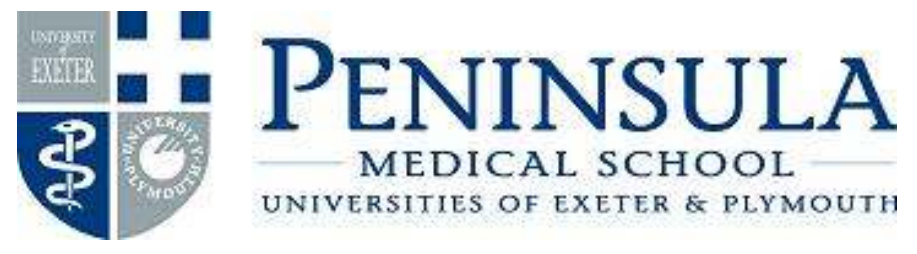

\title{
Complementary Medicine
}

\author{
The Evidence So Far
}

\section{A documentation of our clinically relevant research 1993 - 2010}

(Last updated: January 2011)

Complementary Medicine

Peninsula Medical School

Universities of Exeter \& Plymouth

25 Victoria Park Road

Exeter EX2 4NT

Websites: http://sites.pcmd.ac.uk/compmed/

http://www.interscience.wiley.com/journal/fact

E-mail: Edzard.Ernst@pms.ac.uk

Tel: +44 (0) 1392424989

Fax: +44 (0) 1392427562 


\section{Contents}

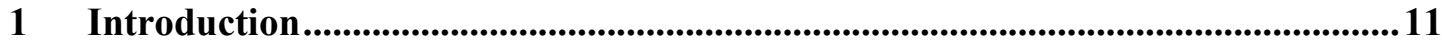

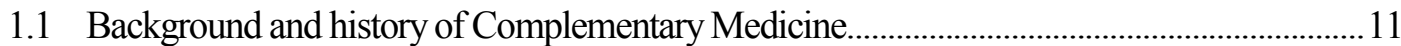

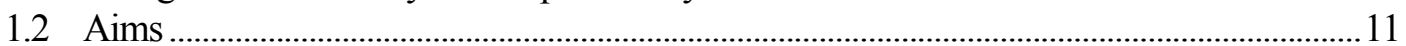

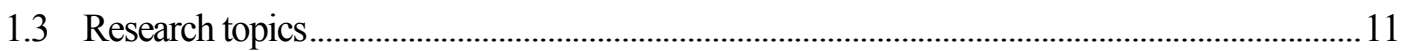

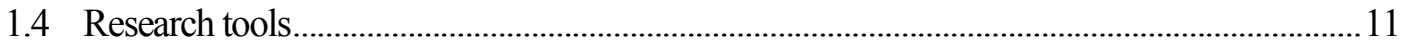

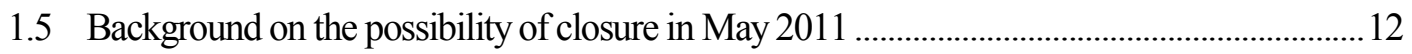

2 The use of complementary medicine (CM) ...............................................................13

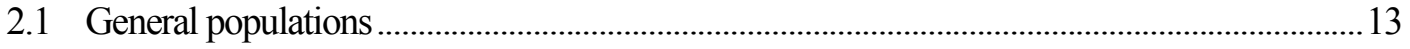

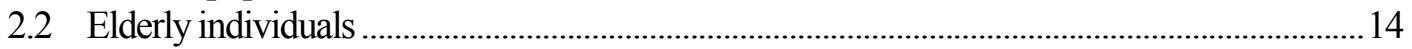

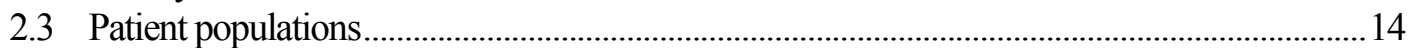

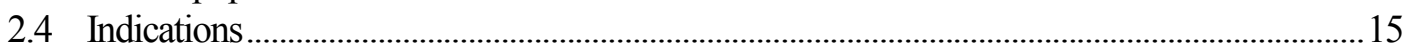

$3 \quad$ Research funding .................................................................................................................16

4 Effectiveness of complementary therapies in specific conditions ............................17

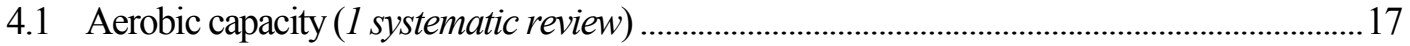

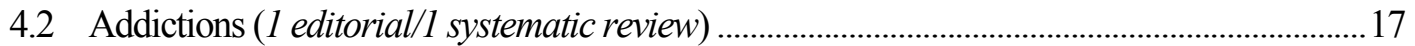

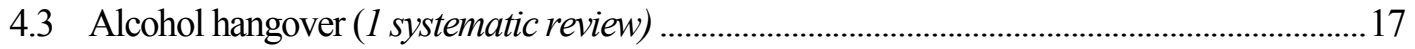

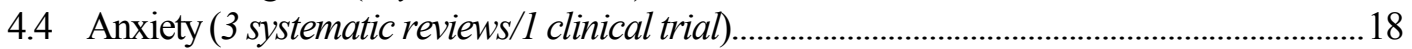

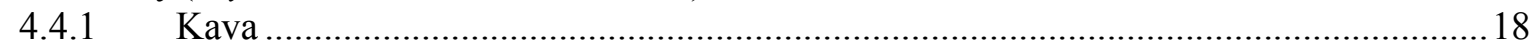

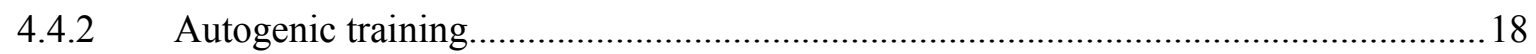

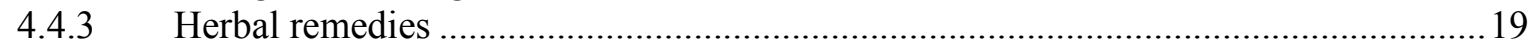

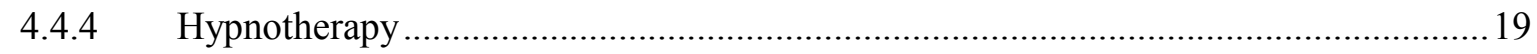

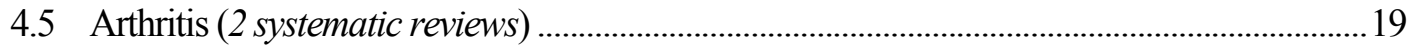

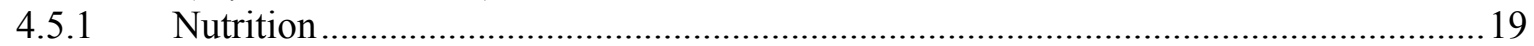

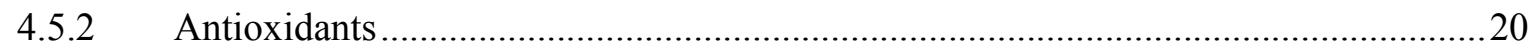

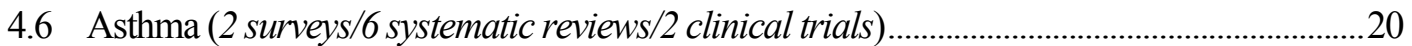

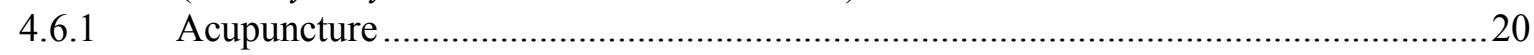

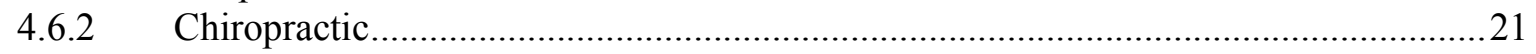

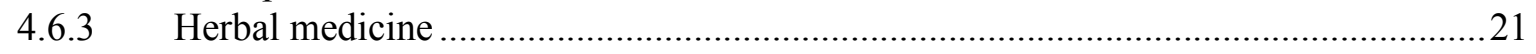

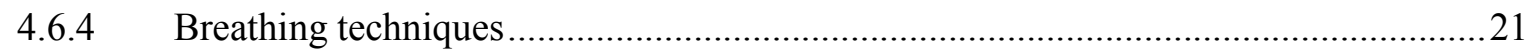

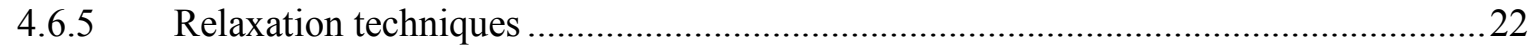

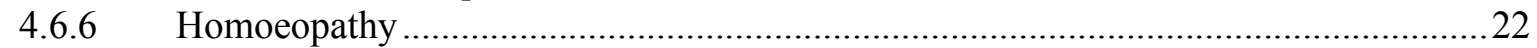

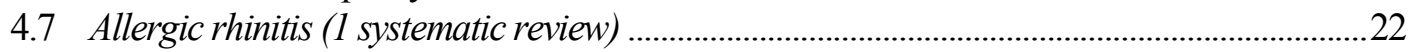

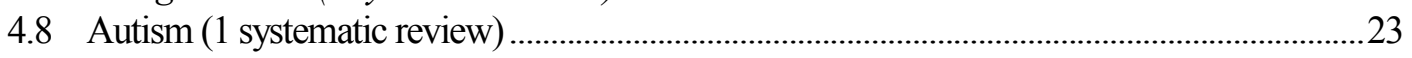

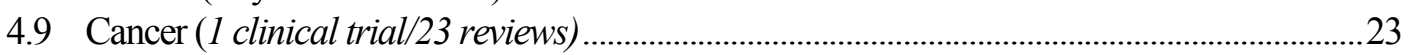

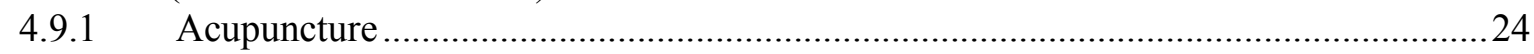

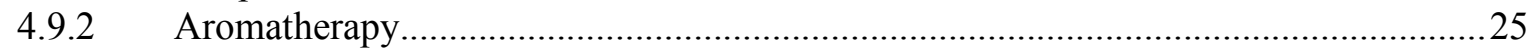

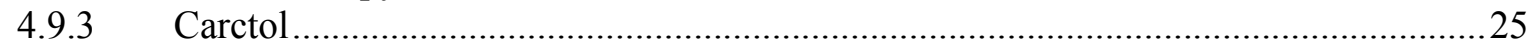

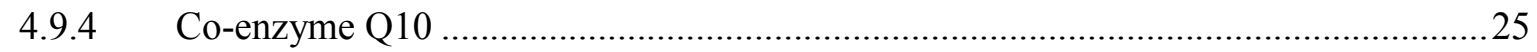

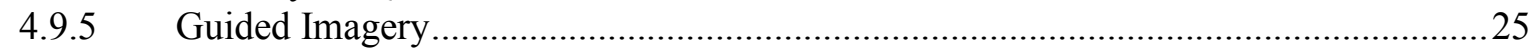

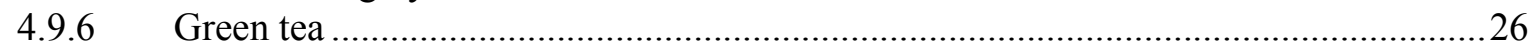

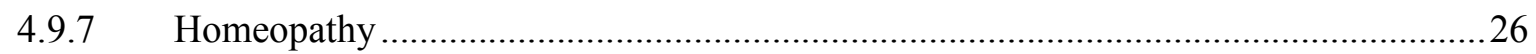

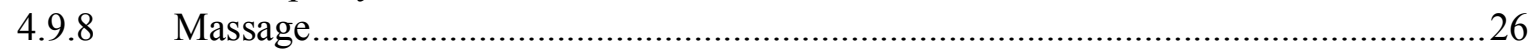

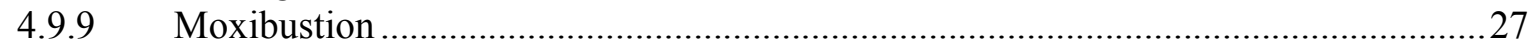

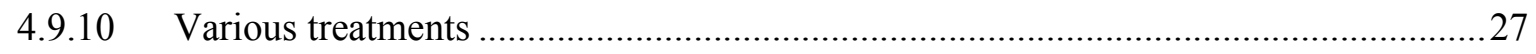

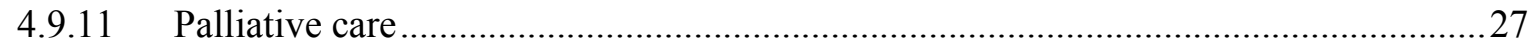

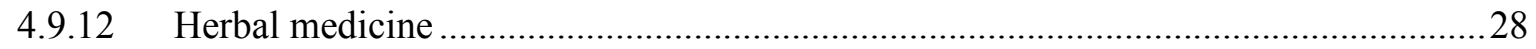

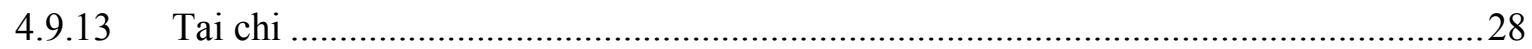

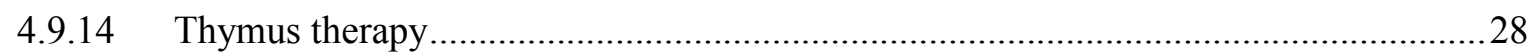




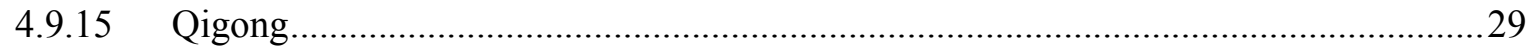

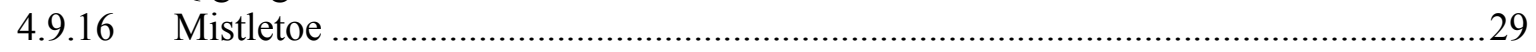

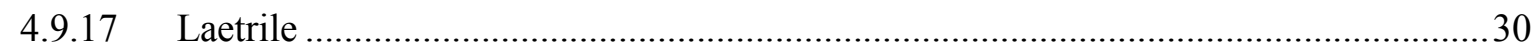

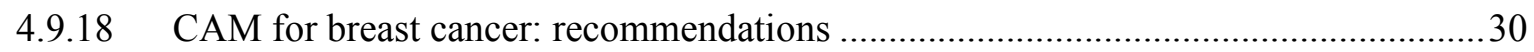

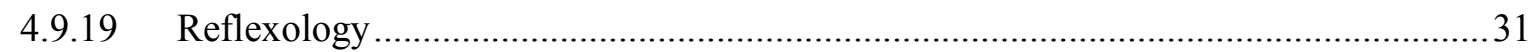

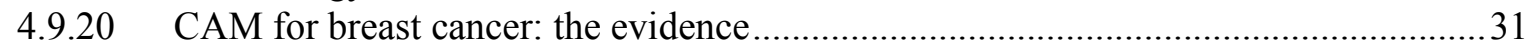

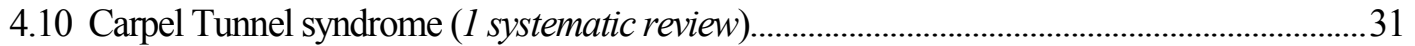

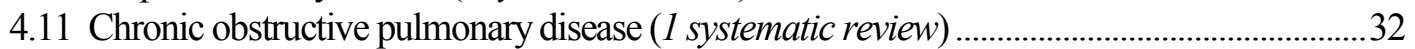

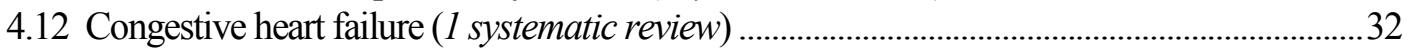

4.13 Cognitive function of healthy volunteers (1 clinical trial/3 systematic reviews) .......................33

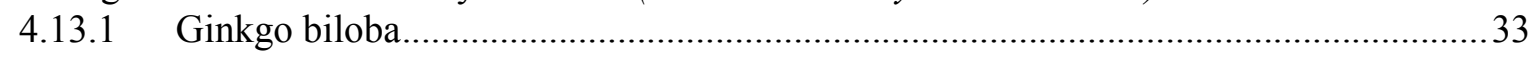

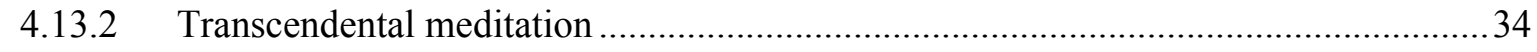

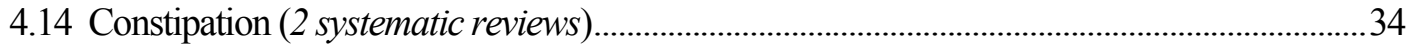

4.15 Coronary heart disease etc (2 clinical trials $/ 5$ systematic reviews)............................................... 34

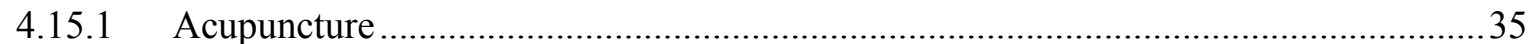

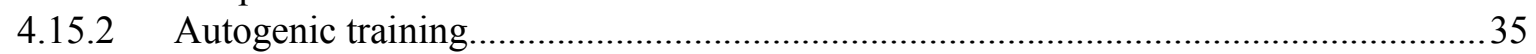

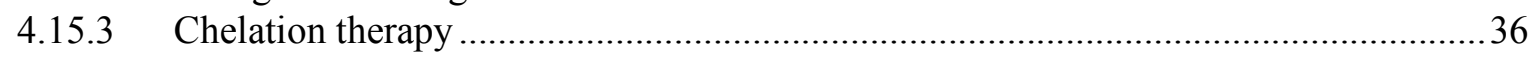

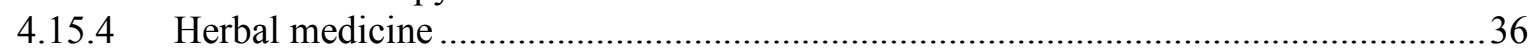

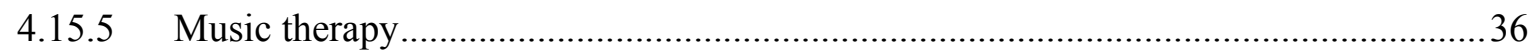

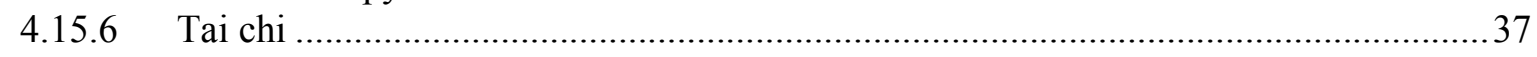

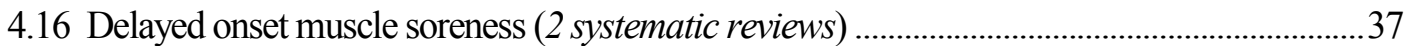

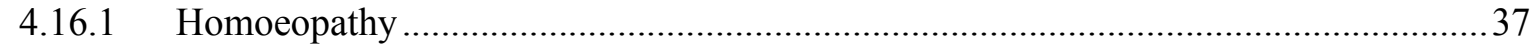

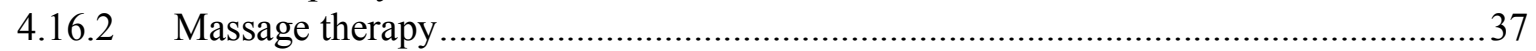

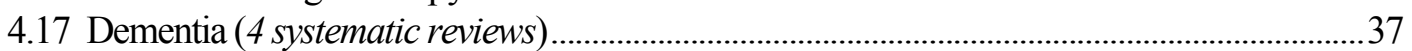

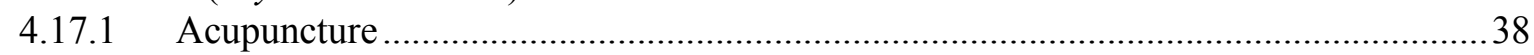

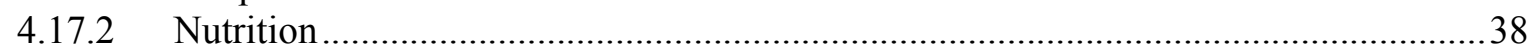

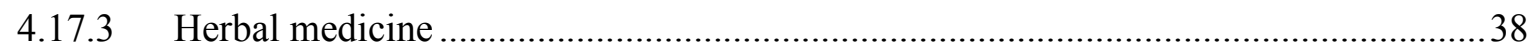

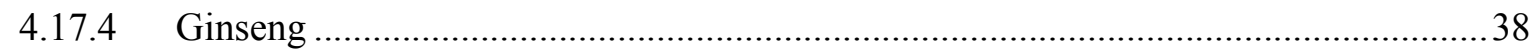

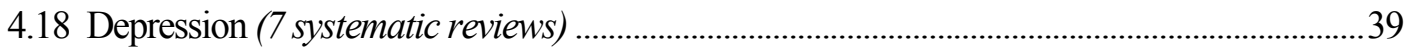

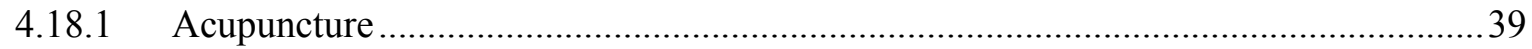

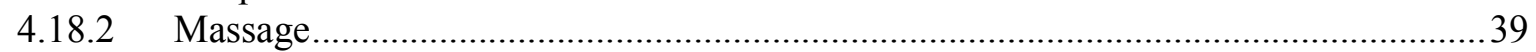

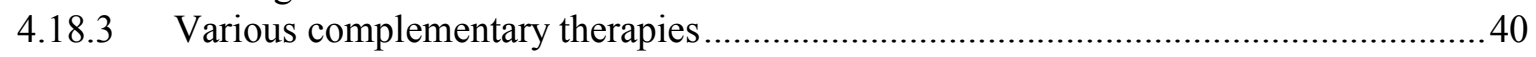

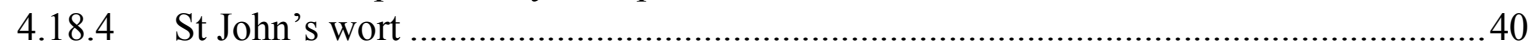

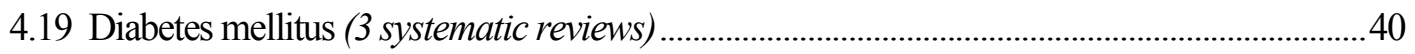

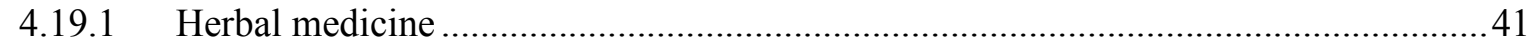

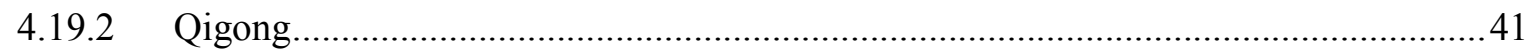

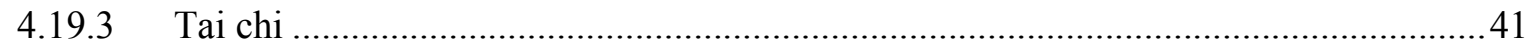

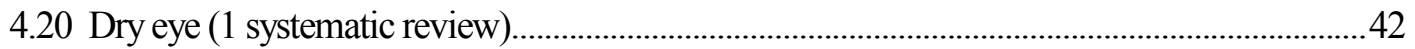

4.21 Eczema (2 systematic reviews, 2 clinical trials) ......................................................................... 42

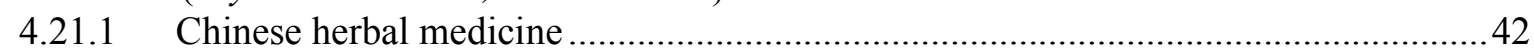

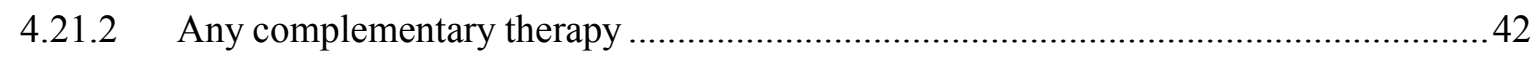

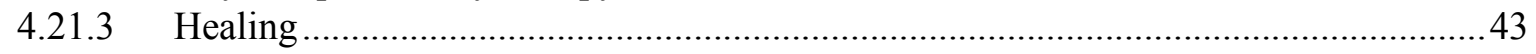

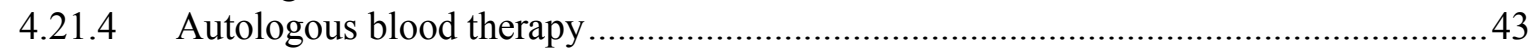

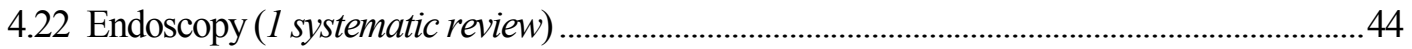

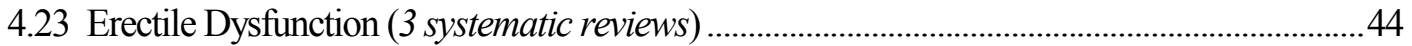

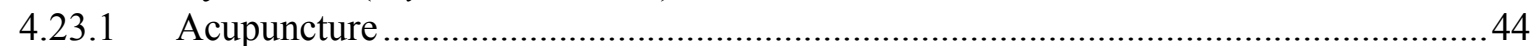

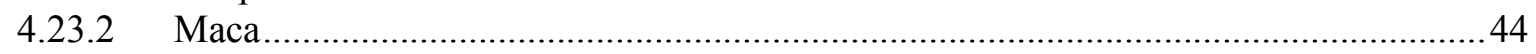

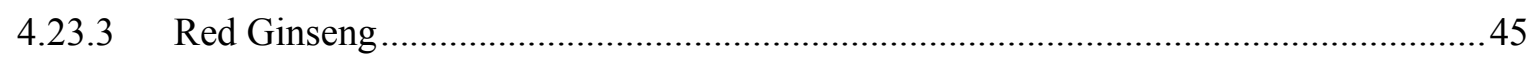

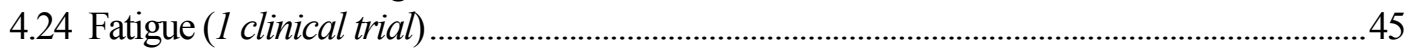

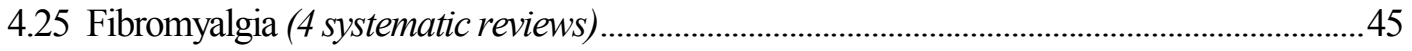

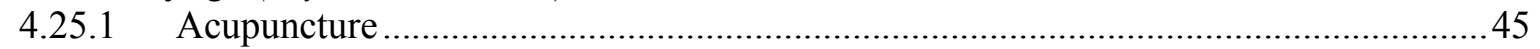




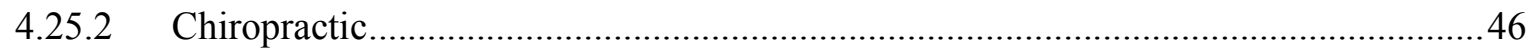

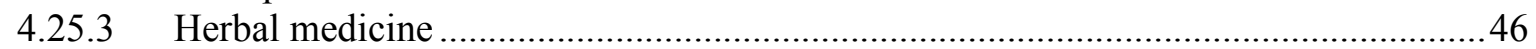

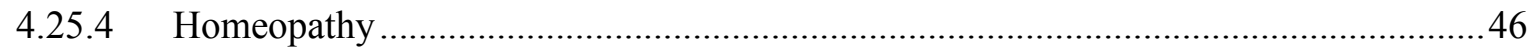

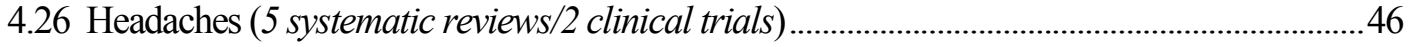

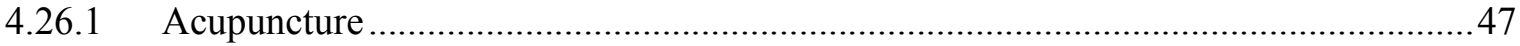

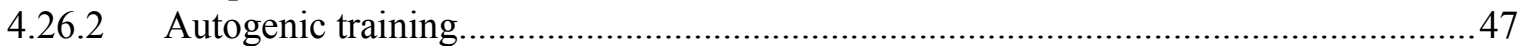

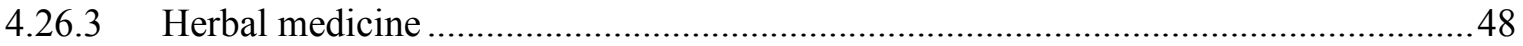

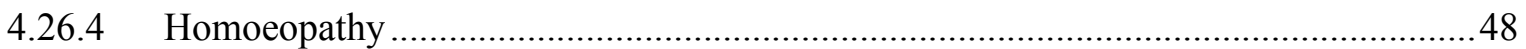

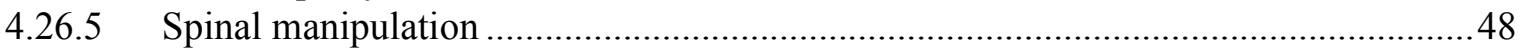

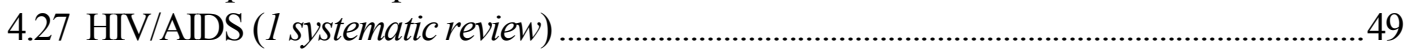

4.28 Hypercholesterolaemia (3 systematic reviews) ............................................................................ 49

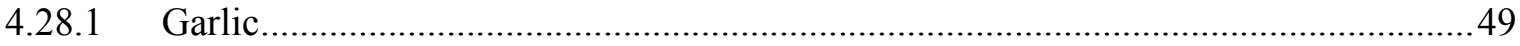

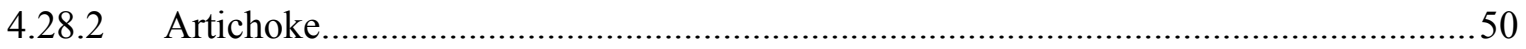

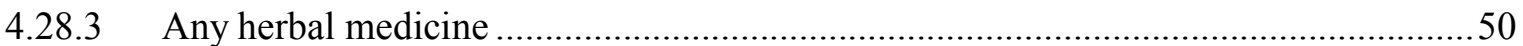

4.29 Hypertension $(9$ systematic reviews)

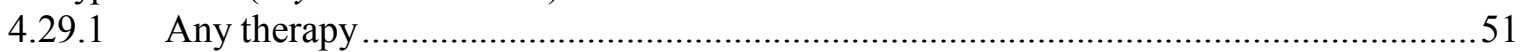

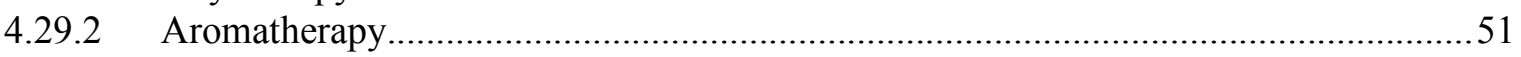

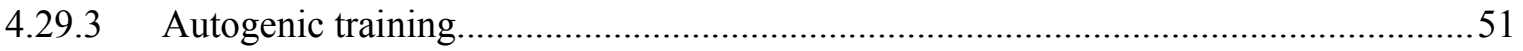

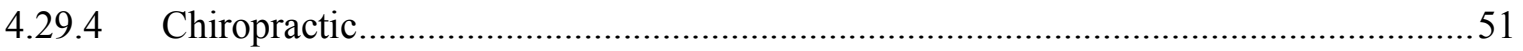

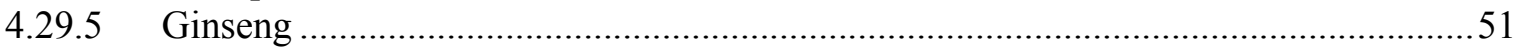

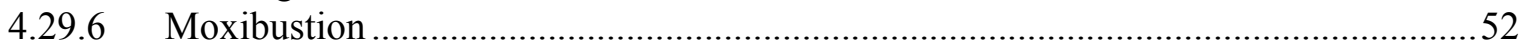

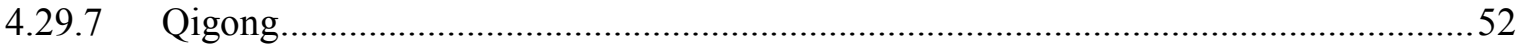

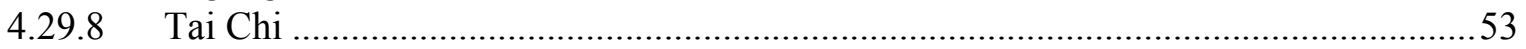

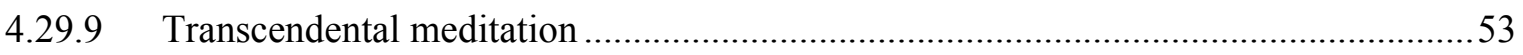

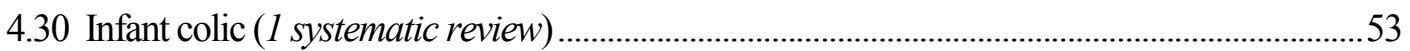

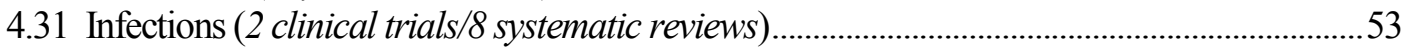

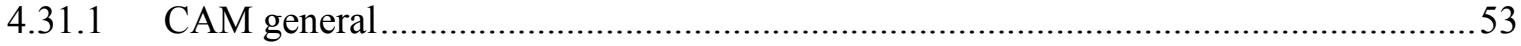

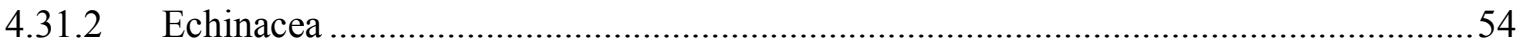

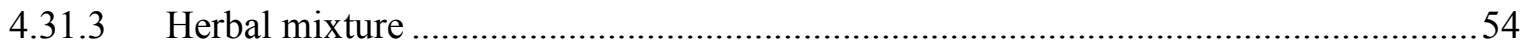

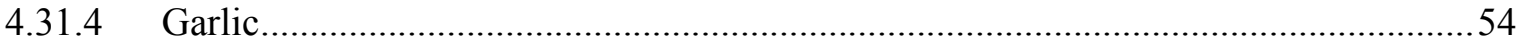

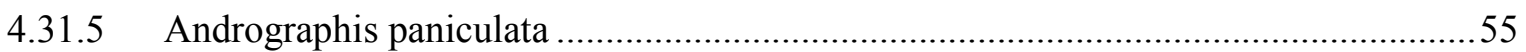

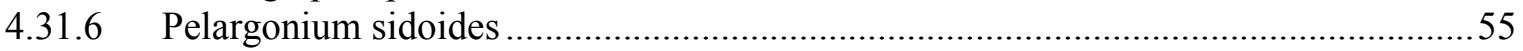

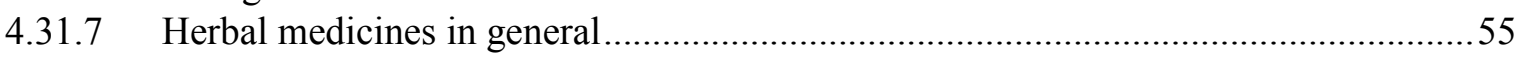

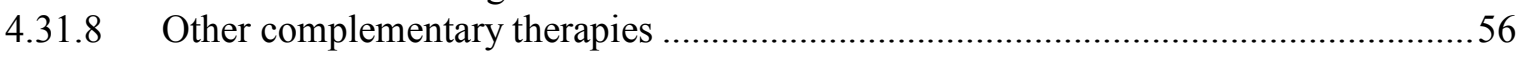

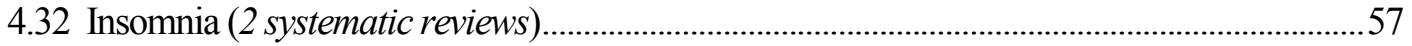

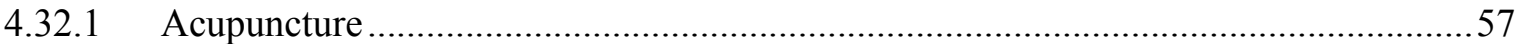

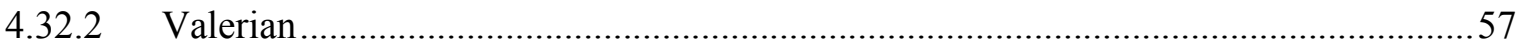

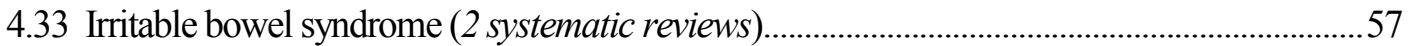

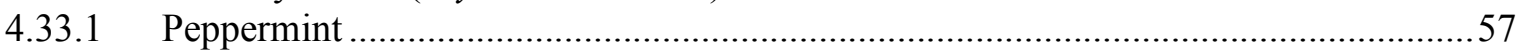

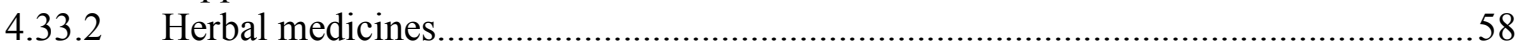

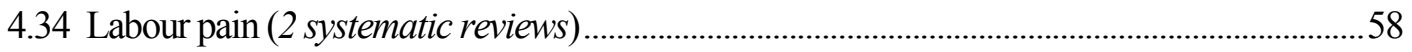

4.35 Low back pain (1 survey/6 systematic reviews/1 editorial)........................................................59

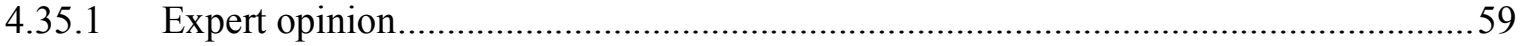

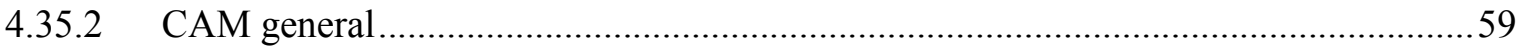

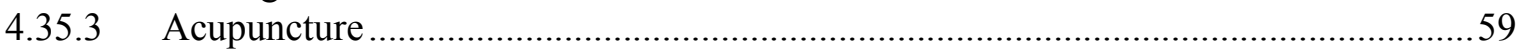

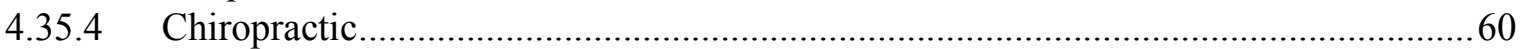

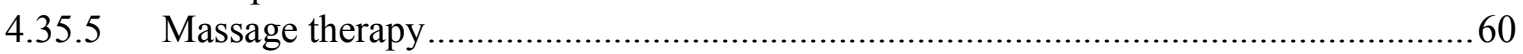

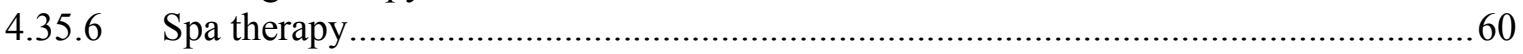

4.36 Menopausal symptoms ( 7 systematic reviews/1 clinical trial) .............................................61

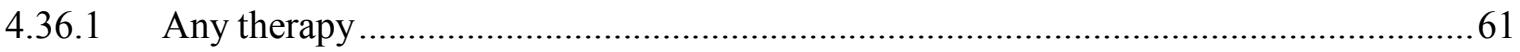

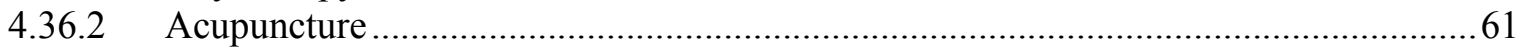


4.36.3 Herbal treatments often recommended for menopausal symptoms ..........................61

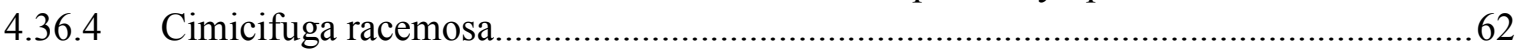

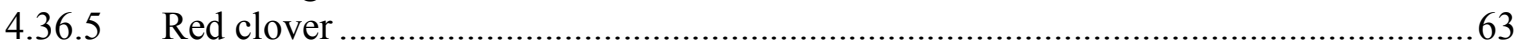

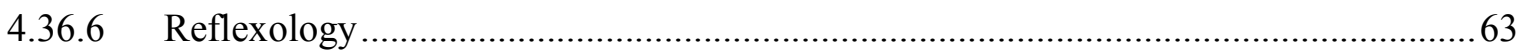

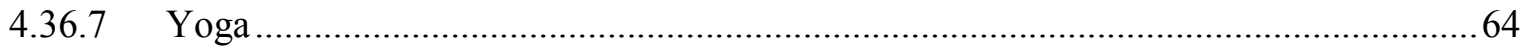

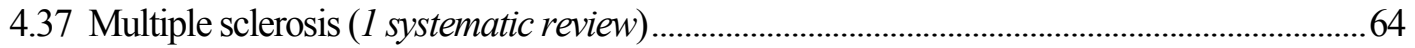

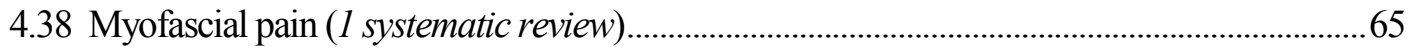

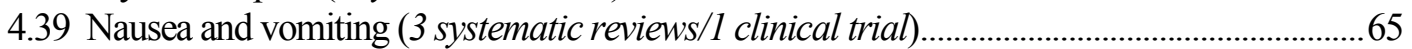

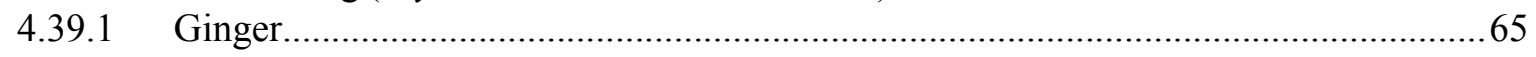

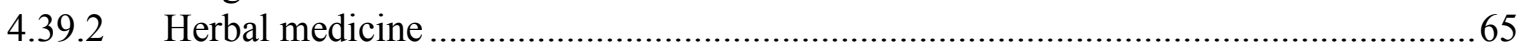

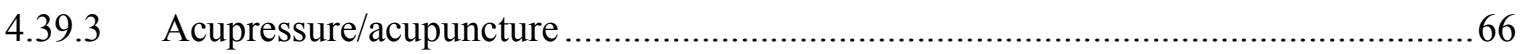

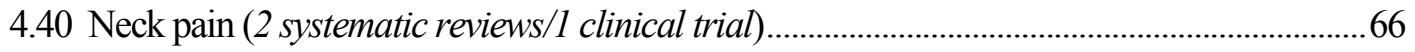

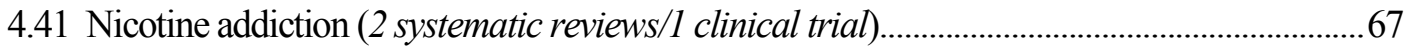

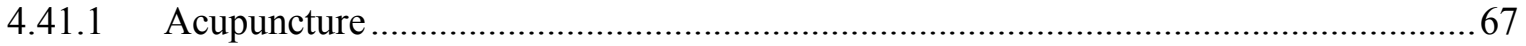

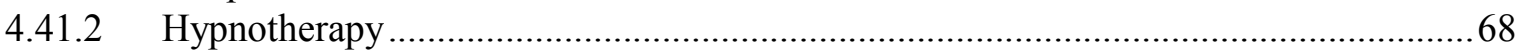

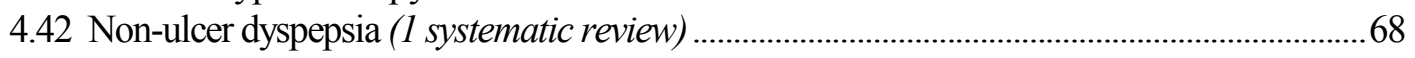

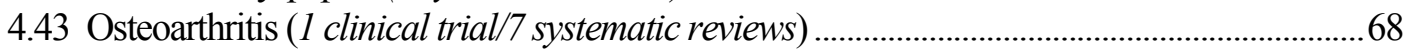

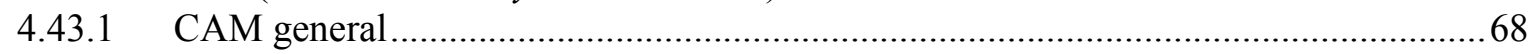

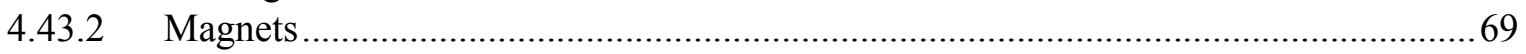

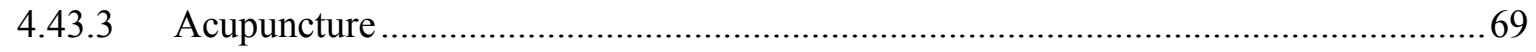

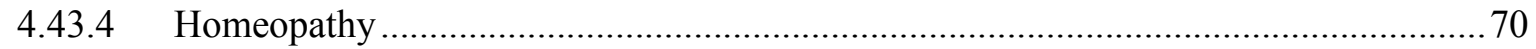

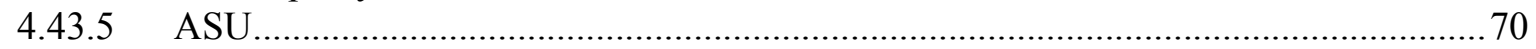

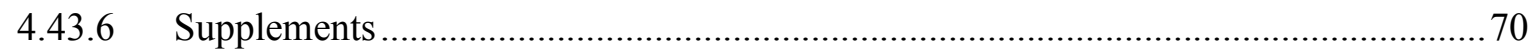

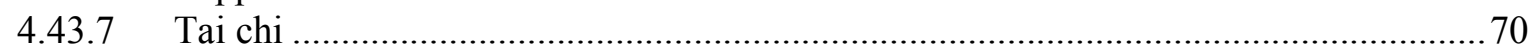

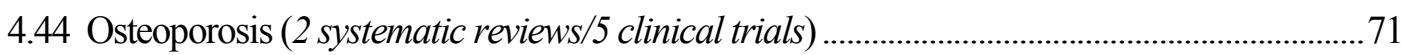

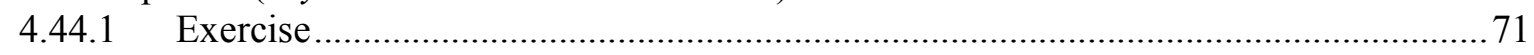

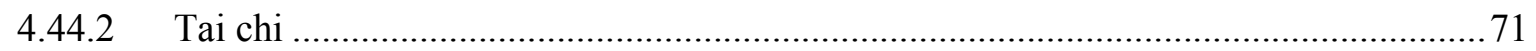

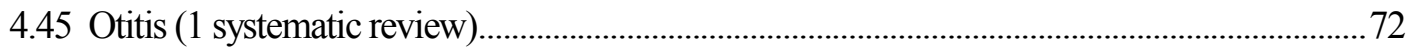

4.46 Other pain syndromes (13 systematic reviews/2 clinical trials/ 1 case report)......................... 72

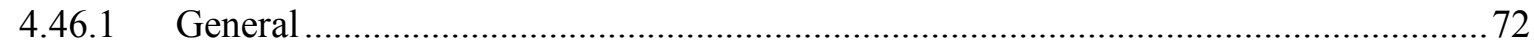

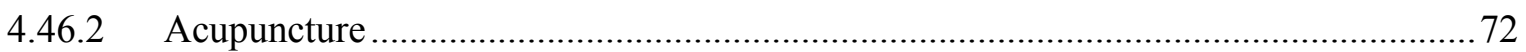

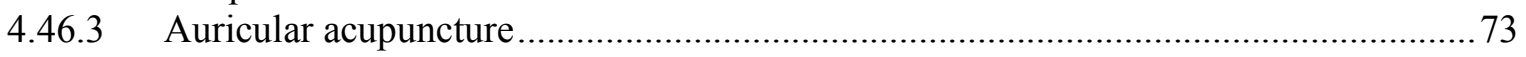

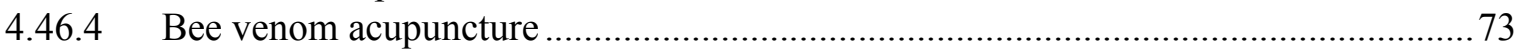

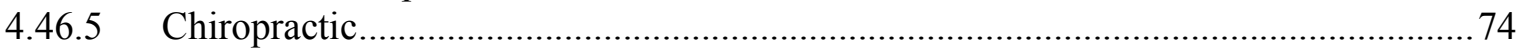

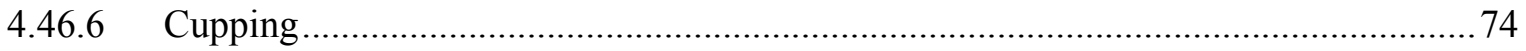

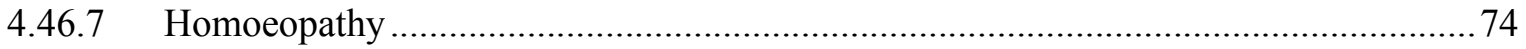

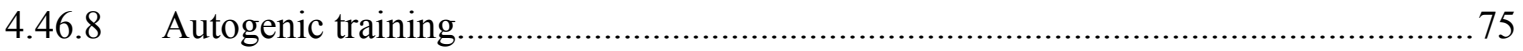

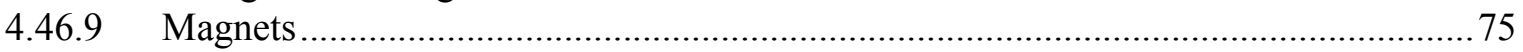

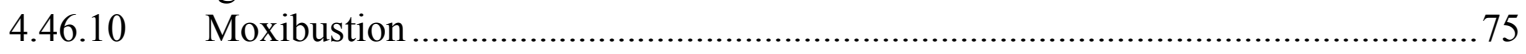

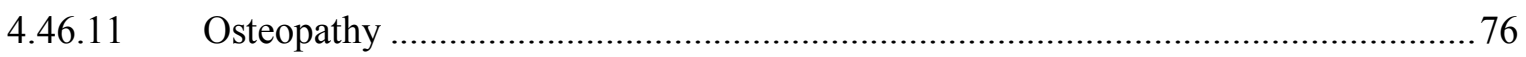

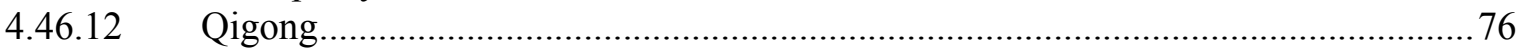

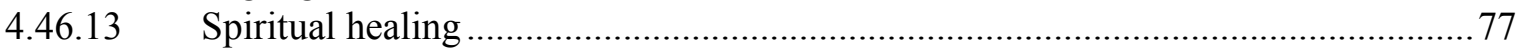

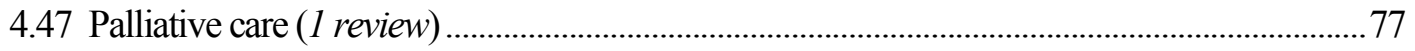

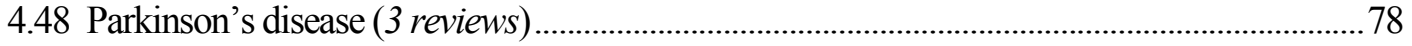

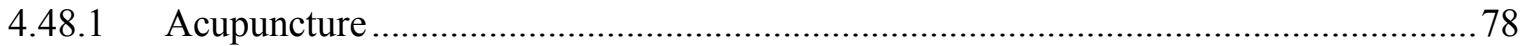

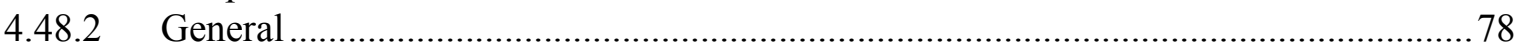

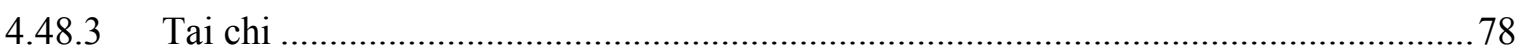

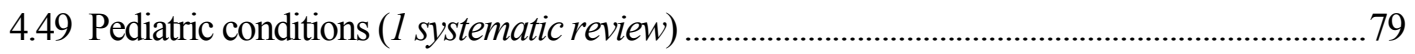

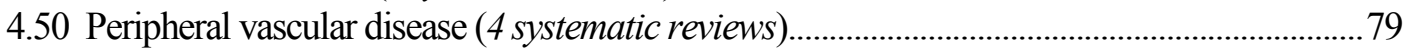

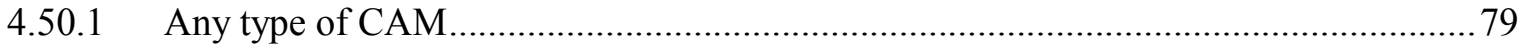

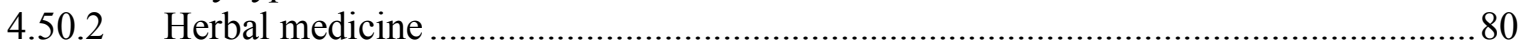




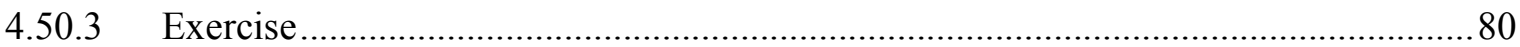

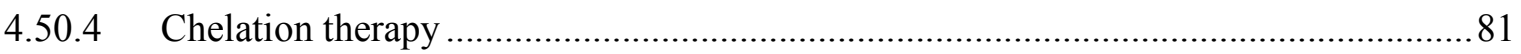

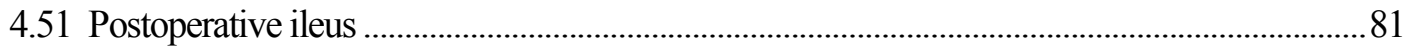

4.52 Premenstrual syndrome (1 systematic review/ 1 clinical trial) ..................................................... 81

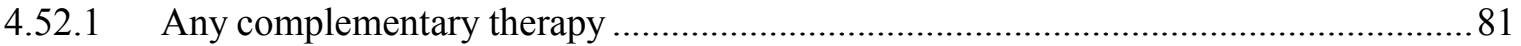

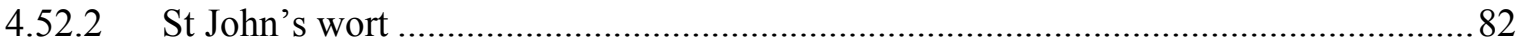

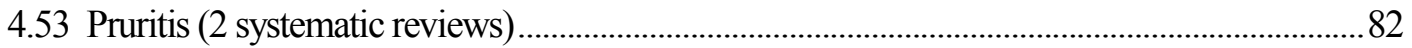

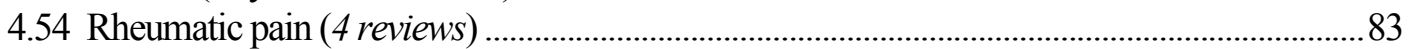

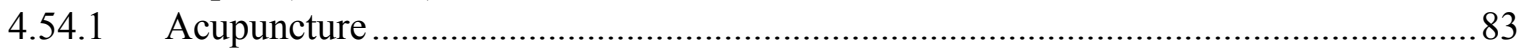

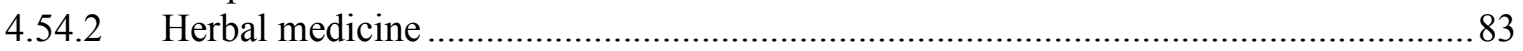

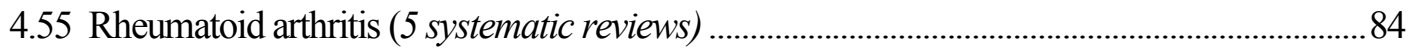

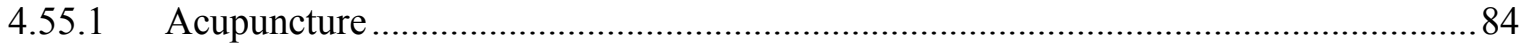

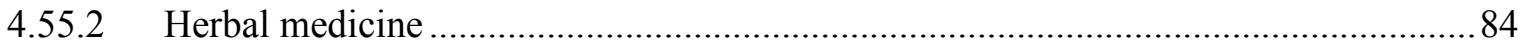

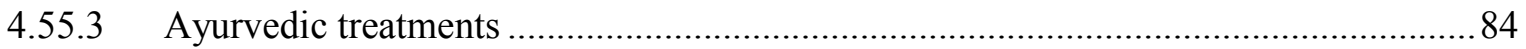

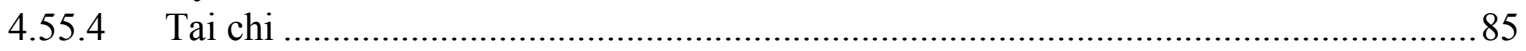

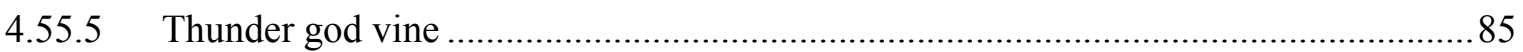

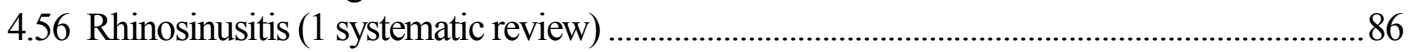

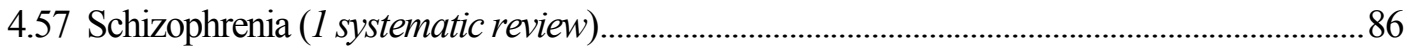

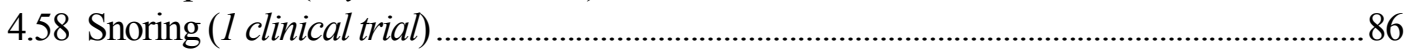

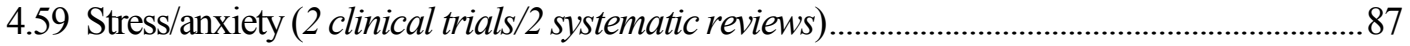

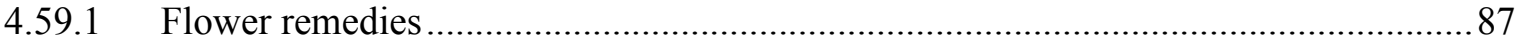

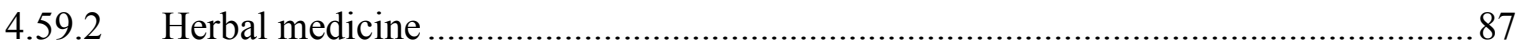

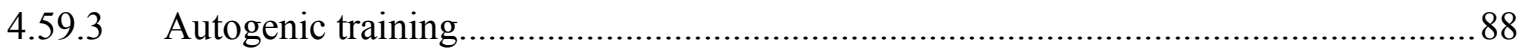

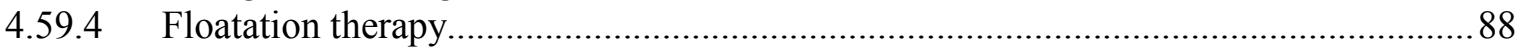

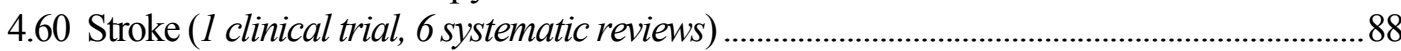

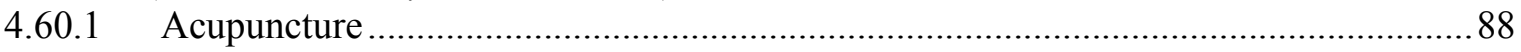

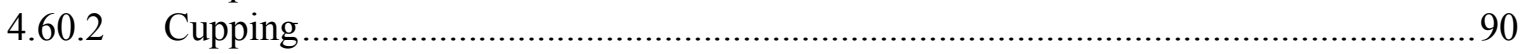

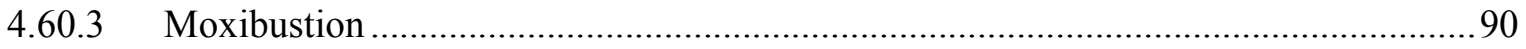

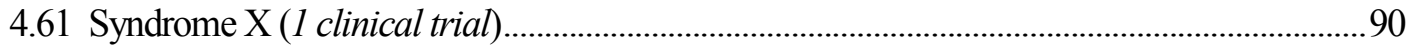

4.62 Temporomandibular joint dysfunction (1 systematic review)................................................91

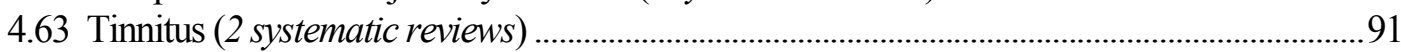

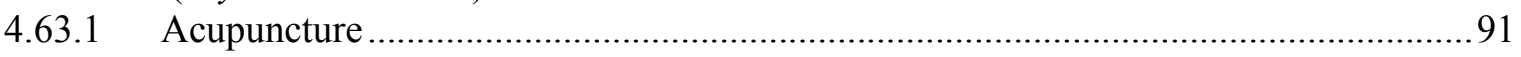

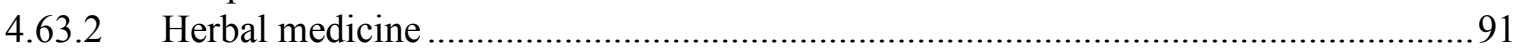

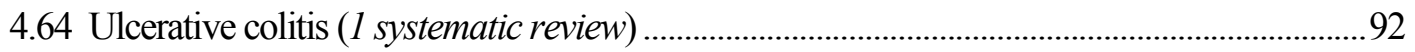

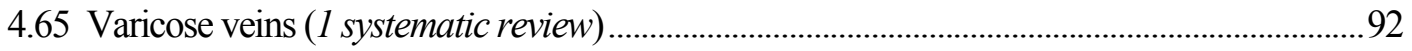

4.66 Weight reduction (2 clinical trials/7 systematic reviews)......................................................92

\begin{tabular}{|c|c|}
\hline 4.66 .1 & upuncture.. \\
\hline 4.66 .2 & Any type of CAM \\
\hline 4.66 .3 & Food supplements ...... \\
\hline 6.4 & Chitosan . \\
\hline 6 & Chromium \\
\hline & Garcinia.. \\
\hline 6.7 & Juar gum... \\
\hline
\end{tabular}

5 Effectiveness/effects/validity of CM modalities (unrelated to specific conditions) 95

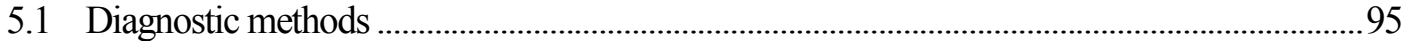

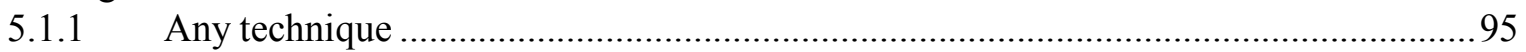

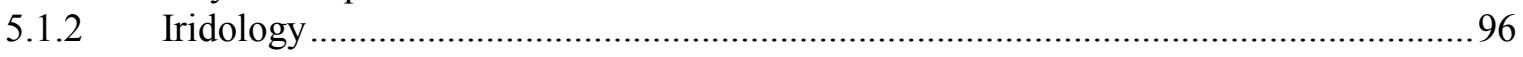

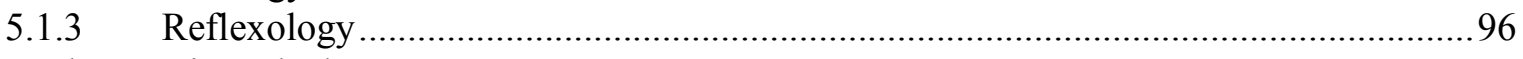

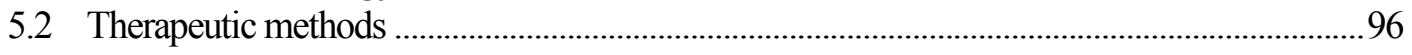

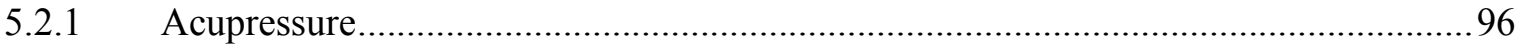

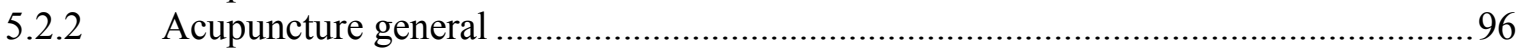




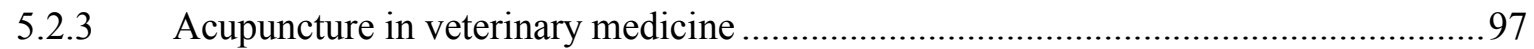

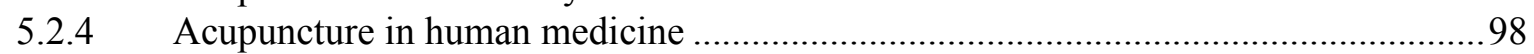

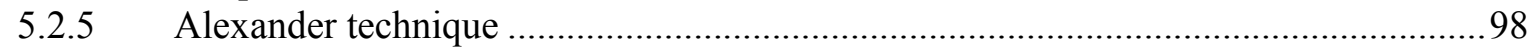

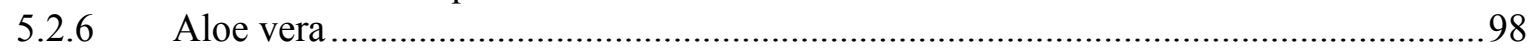

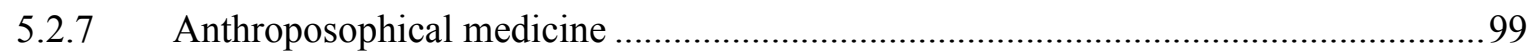

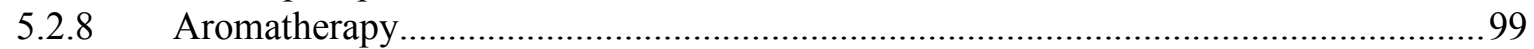

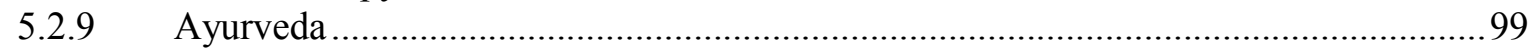

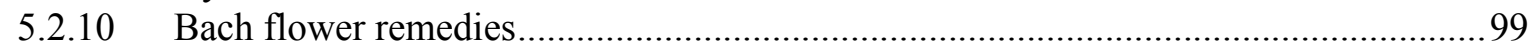

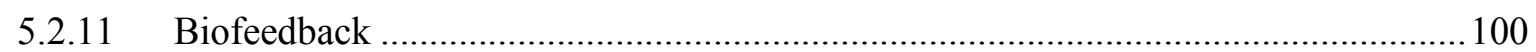

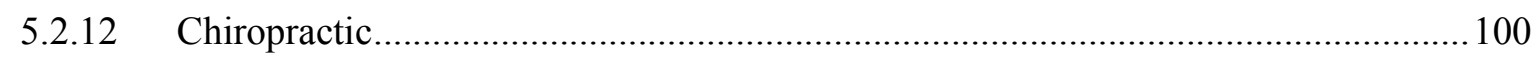

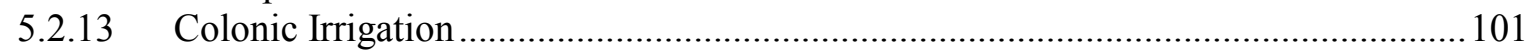

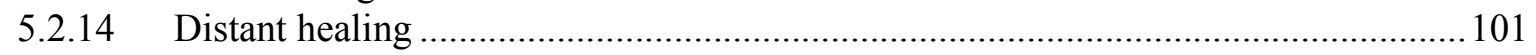

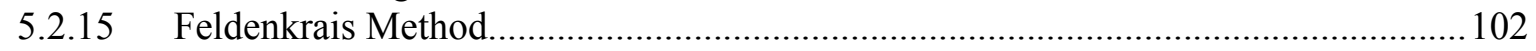

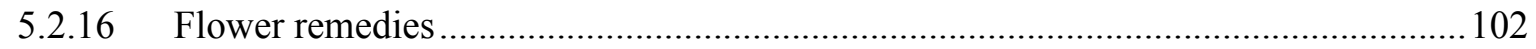

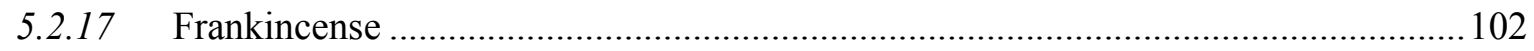

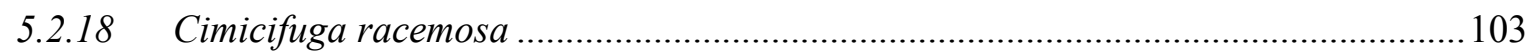

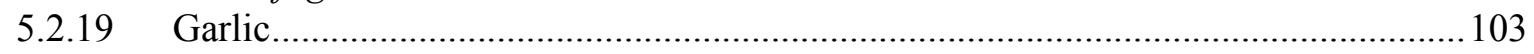

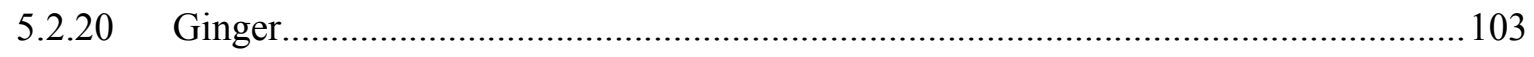

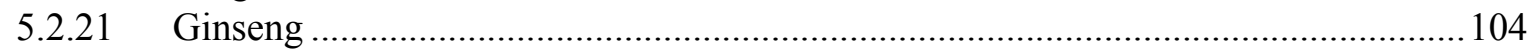

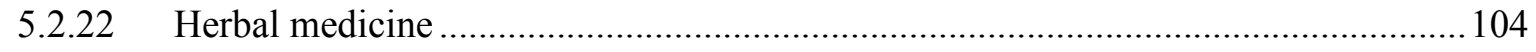

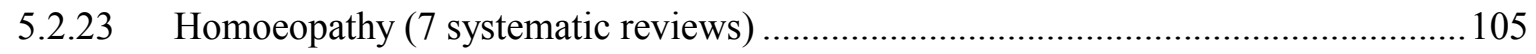

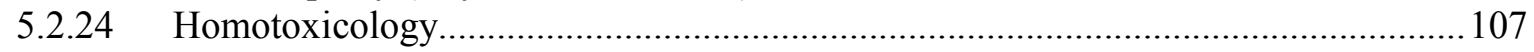

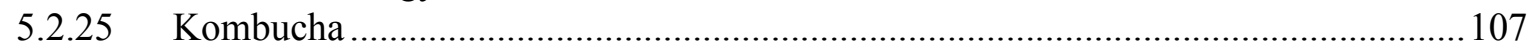

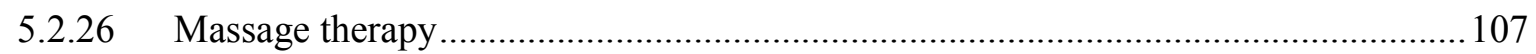

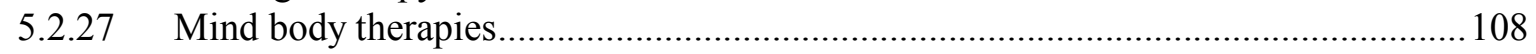

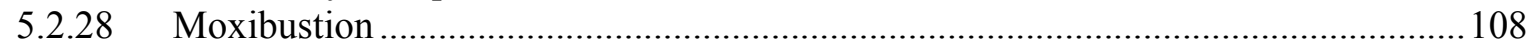

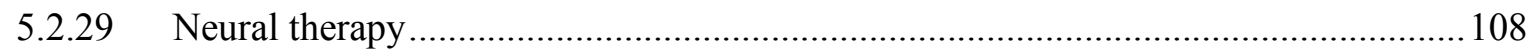

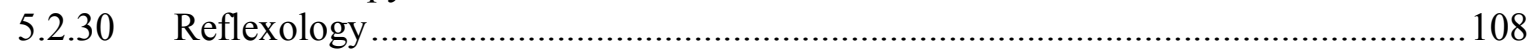

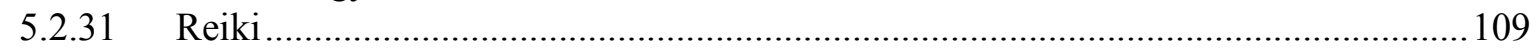

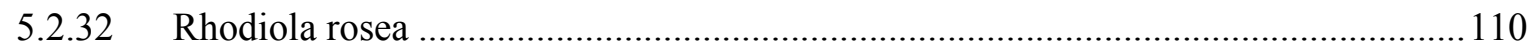

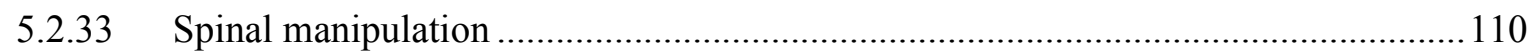

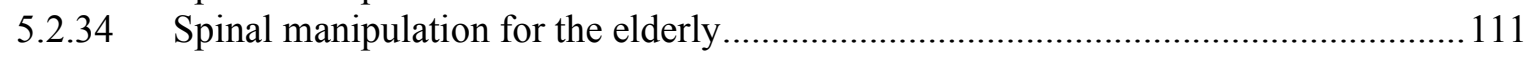

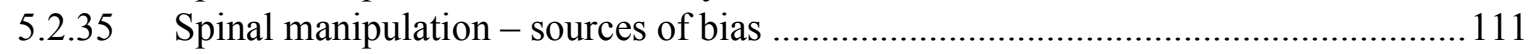

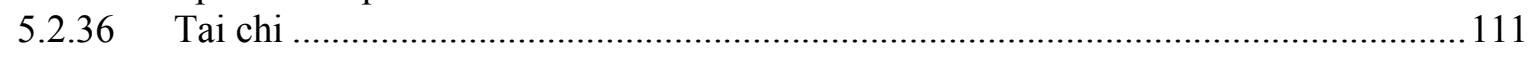

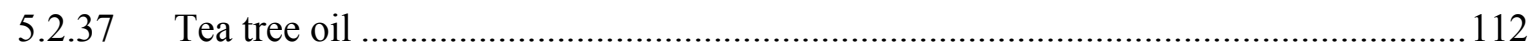

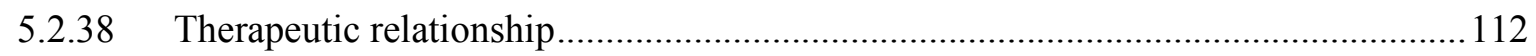

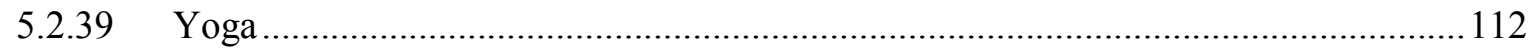

6 Safety of complementary therapies .................................................................................. 113

6.1 Quality of case reports (1 systematic review) .........................................................................113

6.2 Toxicity of herbal medicinal products (HMPs) in general (4 systematic reviews) ................113

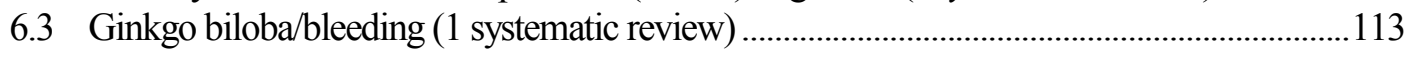

6.4 Hepatotoxicity of herbal medicinal products (1 systematic review).........................................114

6.5 Herbal supplements for body weight reduction (1 systematic review) ......................................114

6.6 Cardiovascular adverse effects of HMPs (1 systematic review) ............................................114

6.7 Psychiatric \& neurological adverse effects of herbal medicines (1 systematic review) .........115

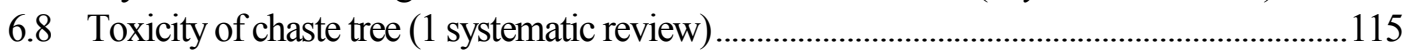

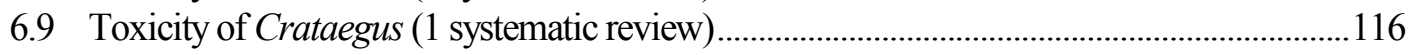

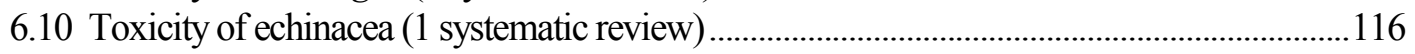

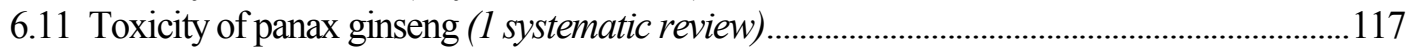

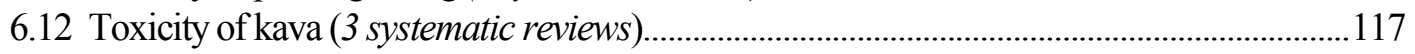

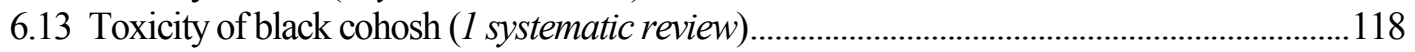


6.14 Toxicity of saw palmetto (1 systematic review)

6.15 Adverse effect of HMPs in pregnancy (1 systematic review) ................................................119

6.16 Adverse effects of unconventional therapies in children (1 systematic review)......................119

6.17 Adverse effects of HMPs in dermatology (1 systematic review)...........................................120

6.18 HMPs' influence on blood coagulation (1 systematic review/1 survey)..................................120

6.19 Herb-drug interactions (8 systematic reviews/1 editorial/2 surveys).......................................120

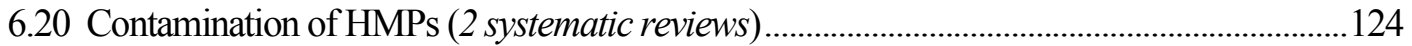

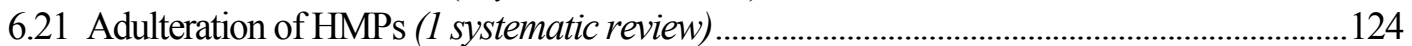

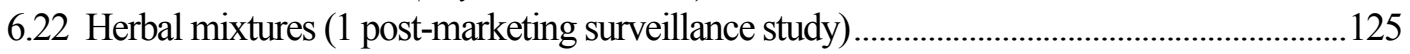

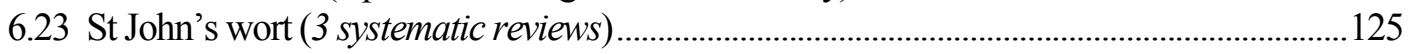

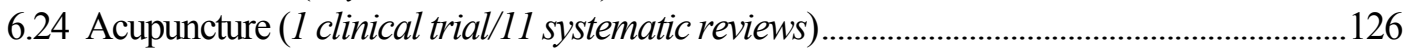

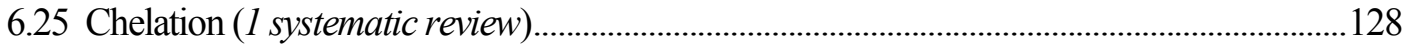

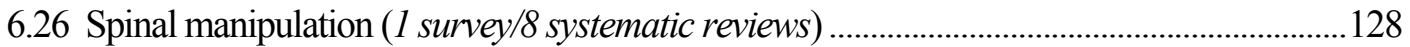

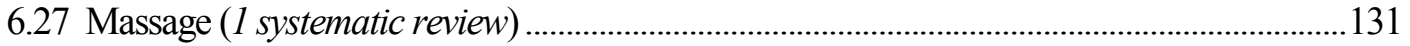

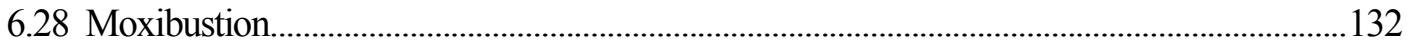

6.29 Safety of therapists (6 surveys/6 systematic reviews/1 editorial) ............................................132

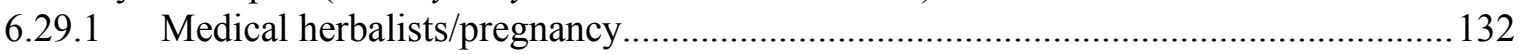

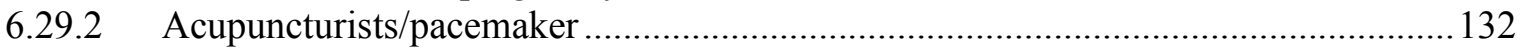

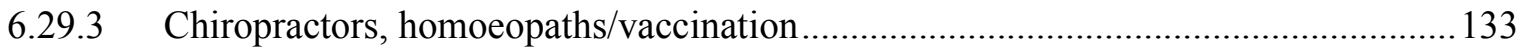

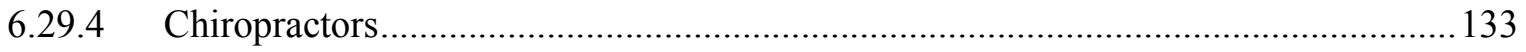

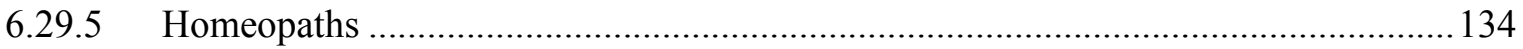

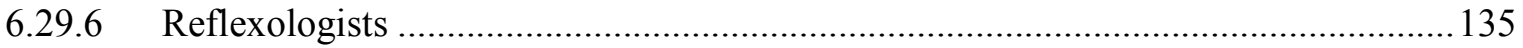

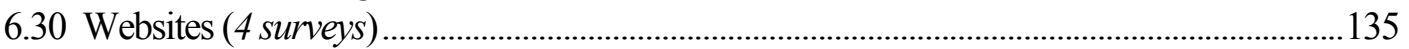

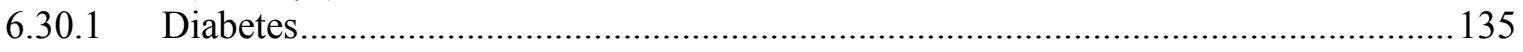

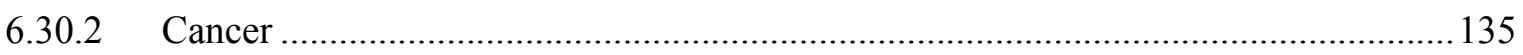

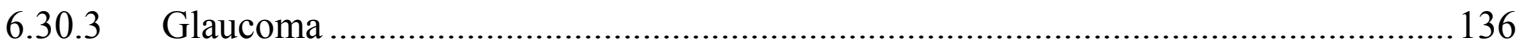

7 Costs of complementary therapies ( 5 systematic reviews) ........................................ 136

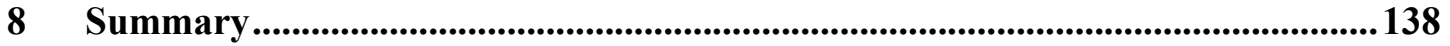

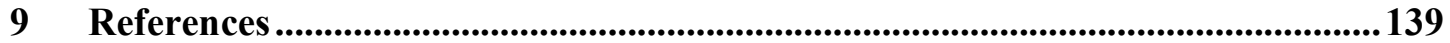




\section{Introduction}

This document provides a brief summary of the most important clinical research findings from Complementary Medicine at the Peninsula Medical School, Universities of Exeter \& Plymouth. Nonclinical research (e.g. development of a placebo acupuncture needle or work on publication bias) has been excluded. A full list of publications is available on request.

We have been updating this report for many years. This version, however, might well be the final one: We have been told that the Exeter University might close us down in May 2011. Even though we have, for over a year, tried everything imaginable to clarify this crucial issue, we have so far (Feb 2011) not received an official notification from our peers (see below).

\subsection{Background and history of Complementary Medicine}

The Laing Chair in Complementary Medicine was established in 1993. Its financial basis is provided by two major endowments from the Maurice Laing Foundation. The Clinical Research Unit was founded in 1994 and the Department of Complementary Medicine in 1996. In 2002 we joined the Peninsula Medical School, Universities of Exeter \& Plymouth, as 'Complementary Medicine'. Today our unit employs a multidisciplinary team of researchers and administrative staff with wide-ranging areas of expertise and professional backgrounds.

\section{$1.2 \quad$ Aims}

- To conduct rigorous, inter-disciplinary and international collaborative research into the efficacy, safety and costs of complementary medicine (CM).

- To be neither promotional nor derogatory but to struggle for objectivity.

- To promote analytical thinking in this area.

- The overreaching aim of all of our research is to maximise the benefit and minimise the risk of $\mathrm{CM}$ for the patient.

Our commitment is primarily to patients and good science.

\subsection{Research topics}

The unit has a dual strategy. Firstly we focus on the most prevalent complementary therapies in the UK:

- acupuncture

- herbalism (phytotherapy)

- homoeopathy

- manipulative therapies (osteopathy and chiropractic).

Secondly we try to cover CM as broadly as possible. Depending on funding, we might therefore conduct research into areas not listed above.

\section{$1.4 \quad$ Research tools}

Our main research tools are systematic reviews, meta-analyses, clinical trials, epidemiological techniques and laboratory investigations. 


\subsection{Background on the possibility of closure in May 2011}

In 1993, the University of Exeter accepted a donation from Sir Maurice Laing and signed a legal contract to contribute the same amount towards the research of the unit. The precise wording used was "the University undertakes to use its best endeavours, beginning immediately, to raise a further capital sum of $£ 1.5$ million". In the same year, Professor Ernst was appointed as Laing Chair of Complementary Medicine. He accepted the post with the understanding that the university would provide $£ 3.0$ million to run the unit initially for 10 years.

Initially, some fund-raising activities were initiated (which Sir Maurice funded with an extra $£ 10,000)$, but they were not very successful. Soon it became clear that the donation would not last. Therefore Professor Ernst approached Sir Maurice for more money. In 2003, Sir Maurice wrote a further cheque for $£ 0.5$ million leaving no doubt about his dissatisfaction with Exeter University's fund-raising efforts and insisting that forthwith "strenuous efforts...be made to secure core funding of the chair...". Since August 2004, the Peninsula Medical School (PMS) has started contributing $£ 50,000$ towards running my unit. This amount is roughly what we have to pay in rent for our premises (not covered by my University or the PMS!) and is nowhere near the promised sum.

After the Prince Charles' Private Secretary filed a complaint with the Vice Chancellor, in September 2005, alleging a breach of confidentiality by Professor Ernst, he was investigated by his administration. At the end of this 13-months long inquisition, the Vice Chancellor wrote that "it would not be appropriate to issue... a formal disciplinary warning...".

At that stage, Professor Ernst felt that he had become 'persona non grata' at the university. No more serious attempts to raise funds for the unit were made. When Professor Ernst wrote to the Vice Chancellor about the ethical obligation to fulfil the promise of the contract (see above), he received no reply. 


\section{The use of complementary medicine (CM)}

\subsection{General populations}

Several of our research projects have indicated that the prevalence of use of CM is high. A recent survey based on a large, representative sample of the British population showed that one in five individuals of the general British population use at least one type of CM within a year. ${ }^{1}$

A survey of general practitioners in South West England ${ }^{2}$ showed that $68 \%$ of the 461 respondents had been involved in CM in some way during the previous week. $16 \%$ practised some form of CM themselves, $25 \%$ had referred at least one patient to a $\mathrm{CM}$ provider and $55 \%$ had endorsed or recommended CM.

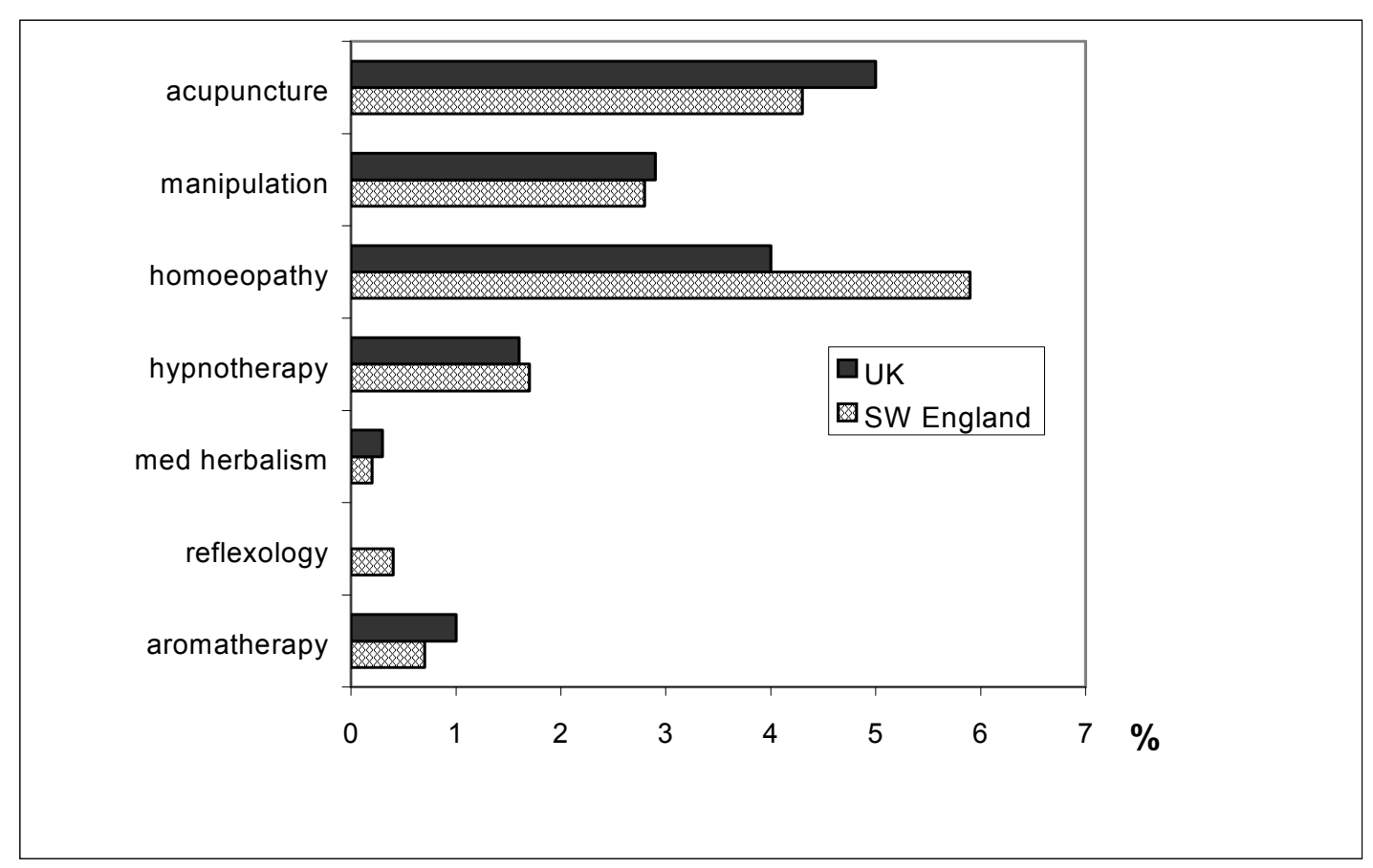

Percentages of GPs in Devon and Cornwall who practise complementary therapy compared with the UK average ('manipulation' included all manipulative techniques).

A similar study of complementary practitioners ${ }^{3}$ suggested that CM providers have a strongly positive attitude towards working within the NHS. Their minimum acceptable fee varied from $£ 20$ $£ 60$ per hour. It was extrapolated that the nationwide integration of CM into the NHS would involve considerable costs.

In many countries, recent data on the use of complementary and alternative medicine (CAM) are available. ${ }^{4}$ However, in England, there is a paucity of such data. We sought to determine the prevalence and predictors of CAM use in England. Data were obtained from the Health Survey for England 2005, a national household survey that included questions on CAM use. We used binary logistic regression modelling to explore whether demographic, health and lifestyle factors predict CAM use. Data were available for 7630 respondents (household response rate $71 \%$ ). Lifetime and 12-month prevalence of CAM use were $44.0 \%$ and $26.3 \%$ respectively; $12.1 \%$ had consulted a practitioner in the preceding 12 months. Massage, aromatherapy and acupuncture were the most commonly used therapies. Twenty-nine percent of respondents taking prescription drugs had used CAM in the last 12 months. Women (OR $0.491,95 \%$ CI: $0.419,0.577$ ), university educated 
respondents (OR 1.296, 95\% CI: 1.088, 1.544), those suffering from anxiety or depression (OR $1.341,95 \%$ CI: 1.074, 1.674), people with poorer mental health (on GHQ: OR 1.062, 95\% CI 1.026, $1.100)$ and lower levels of perceived social support (1.047, 95\% CI: 1.008, 1.088), people consuming $\$ 5$ portions of fruit and vegetables a day (OR $1.327,95 \%$ CI: $1.124,1.567)$ were significantly more likely to use CAM. In conclusion, complementary and alternative medicine use in England remains substantial, even amongst those taking prescription drugs. These data serve as a valuable reminder to medical practitioners to ask patients about CAM use and should be routinely collected to facilitate prioritisation of the research agenda in CAM.

\subsection{Elderly individuals}

The use of herbal and nutritional supplements by older British people is increasing and despite the risks of adverse effects and negative herb-drug interactions, doctors are often not informed of their use. The number of herbal and nutritional supplements used most frequently by older people, and which particular herbal extracts are used, are unknown. This study ${ }^{5}$ aimed to identify how many herbal and nutritional supplements and which herbal extracts are most frequently used by British people $>50$ years of age, and to identify which herb-drug interactions represent the greatest risk to this age group. A self-completed survey was promoted through websites and a popular magazine aimed at people $>50$ years of age. Respondents $(n=271)$ used a mean of 2.26 prescription drugs and 5.91 herbal and nutritional supplements, including 2.66 herbal extracts. The use of herbal medicines by older British people frequently involves the use of several herbs, often involves concomitant use with several nutritional supplements, is poorly reported to doctors and places older people at risk of negative herb-drug interactions. The most frequently used herbs and associated risks of negative interactions with prescription drugs are identified. Older people should be encouraged to report the use of herbs and nutritional supplements to their doctors, and doctors should be provided with comprehensive and up-to-date information about potential herb-drug interactions.

\subsection{Patient populations}

Specific projects were aimed at assessing CM use in defined patient populations. In general they demonstrated a high degree of acceptance of CM by the following groups:

- Adult patients with asthma (survey in conjunction with National Asthma Campaign). ${ }^{6} 4741$ asthma sufferers participated in this survey. The results show that $59 \%$ of them had tried some form of CM. The most popular treatments were breathing techniques, homoeopathy and herbalism.

- Children with asthma (survey in conjunction with National Asthma Campaign). ${ }^{7} 1471$ caretakers of children with asthma participated in this survey. 33\% of them stated that they had tried CM for their child. The most popular treatments were homoeopathy, breathing techniques and herbalism.

- People suffering from arthritis. ${ }^{8} 1020$ arthritis sufferers participated in this survey. One third of them had received at least one form of CM from a complementary practitioner.

- Dermatological patients. ${ }^{9}$ A systematic review of all surveys was conducted. Seven surveys were included. Lifetime prevalence of CM usage by dermatological patients ranged from $35 \%$ to $69 \%$. The most popular treatments were homoeopathy, herbalism and food supplements.

- Patients infected with $\underline{\mathrm{HIV}} .{ }^{10}$ A systematic review of all surveys in this area included 12 studies. The prevalence of CM use ranged between $27 \%$ and $100 \%$. Vitamins, massage, acupuncture and imagery were among the most popular. 
- Paediatric patients. ${ }^{11}$ A systematic review of all surveys in this area included 10 studies. The prevalence of CM use ranged between 9\% (1997) and 70\% (1990). Homoeopathy and herbalism were the most popular treatments.

- Cancer patients. ${ }^{12}$ A systematic review of all surveys in this area included 26 studies from 13 countries. The prevalence of $\mathrm{CM}$ use ranged from $7 \%$ to $64 \%$ (average $=31 \%$ ). The type of CM treatment varied over time and by nation.

- Rheumatological patients. ${ }^{13}$ A systematic review of all surveys in this area included 14 studies. The prevalence of CM use ranged from $30 \%$ to nearly $100 \%$. Dietary approaches, herbalism and acupuncture were amongst the most popular treatments.

\subsection{Indications}

Our survey data ${ }^{4}$ also showed that some indications attract more CAM usage than others. ${ }^{14}$ The data are summarised in the table below

Prevalence figures according to medical conditions

\begin{tabular}{llll}
\hline Condition & $\begin{array}{l}\text { No. of respondents } \\
\text { with condition }\end{array}$ & $\begin{array}{l}\text { Lifetime prevalence } \\
\text { of CAM use (\%) }\end{array}$ & $\begin{array}{l}\text { 12-month prevalence } \\
\text { of CAM use (\%) }\end{array}$ \\
\hline Anxiety/depression & 1291 & $700(54.2)$ & $452(35.0)$ \\
Pain & 2324 & $1194(51.4)$ & $690(29.7)$ \\
Obesity & 1521 & $716(47.1)$ & $437(28.7)$ \\
Mobility problems & 1263 & $580(45.9)$ & $304(24.1)$ \\
Cancer & 135 & $55(40.7)$ & $24(17.8)$ \\
Asthma & 160 & $61(38.1)$ & $28(17.5)$ \\
Arthritis (including osteoarthritis and & 665 & $246(37.6)$ & $113(17.3)$ \\
rheumatoid arthritis) & 242 & $87(36.0)$ & $38(15.7)$ \\
Angina & 174 & $59(33.9)$ & $27(15.5)$ \\
Arrhythmia & 836 & $262(31.3)$ & $111(13.3)$ \\
Hypertension & 181 & $60(33.1)$ & $22(12.2)$ \\
Myocardial infarction & 121 & $40(33.1)$ & $18(14.9)$ \\
Stroke & 1041 & $335(32.2)$ & $143(13.7)$ \\
Cardiovascular disease* & 120 & $37(30.8)$ & $11(9.2)$ \\
Chronic lung disease & 175 & $42(24.0)$ & $19(10.9)$ \\
Diabetes & & &
\end{tabular}

*Cardiovascular disease to include angina, arrhythmia, hypertension, myocardial infarction, stroke. 


\section{Research funding}

One of the major problems for CM research is research funding. In the UK, the situation is particularly discouraging. A custom-made questionnaire was sent to all UK medical charities in 1999 and again in 2002. ${ }^{15}$ Its primary aim was to assess the commitment of these institutions towards funding research in CM. Sixty-two (1999) and 60 (2002) answers were received corresponding to response rates of $62 \%$ and $55 \%$. The total CM research funds have increased from $£ 70,000$ in 1999 to $£ 412,755$ in 2002 . In terms of total research budgets, this amounts to $0.05 \%$ and $0.31 \%$ respectively. The number of CM research projects has increased but so has the number of charities which do not fund $\mathrm{CM}$ research. We conclude from these data that $\mathrm{CM}$ research funding by UK medical charities has increased. In relative terms it does, however, remain low and out of proportion to the prevalence of CM use in the UK. 


\section{Effectiveness of complementary therapies in specific conditions}

The aim of this article ${ }^{16}$ is to provide a preliminary estimate of how much CAM is evidence-based. For this purpose, I calculated the percentage of 685 treatment/condition pairings evaluated in the 'Desktop Guide to Complementary and Alternative Medicine' which ere supported by sound data. The resulting figure was $7.4 \%$. For a range of reasons, it might be a gross over-estimate. Further investigations into this subject are required to arrive at more representative figures.

\subsection{Aerobic capacity (1 systematic review)}

Tai chi has been claimed to generate beneficial effects with respect to a wide range of diseases. The purpose of this systematic review ${ }^{17}$ was to evaluate evidence from randomized clinical trials testing the effectiveness of tai chi for increasing aerobic capacity. Systematic searches were conducted on fourteen electronic databases without restrictions on population characteristics or the language of publication. The outcome measures considered for inclusion were changes in maximal oxygen consumption as a test for aerobic capacity. Five randomized clinical trials (RCTs) met all inclusion criteria. Three RCTs compared the effects of tai chi with no treatment. The meta-analysis failed to show an effect of tai chi on aerobic capacity compared with sedentary controls $[n=151$, weight mean difference, $\mathrm{ml} / \mathrm{kg} / \mathrm{min}, 0.50,95 \%$ confidence intervals -1.14 to $2.15, \mathrm{P}=0.55$ ]. Two RCTs compared tai chi with conventional physical exercise including brisk, low intensity and moderate intensity walking, and aerobic exercise. The results show that tai chi was not statistically significantly superior to physical exercise. In conclusion, the existing evidence doe not suggest that regular tai chi is an effective way of increasing aerobic capacity.

\subsection{Addictions (1 editorial/1 systematic review)}

An editorial was dedicated to the evidence for or against CM for illicit drug dependence, alcohol dependence and nicotine dependence. ${ }^{18}$ It concluded that to date no form of CM has been identified that could treat any of these conditions effectively.

A systematic review of systematic ${ }^{19}$ reviews included 11 such articles testing the effectiveness of acupuncture for addictions. Most reviews were of poor quality primary studies. The most reliable evidence does not support the notion that acupuncture is an effective therapy for addictions.

\subsection{Alcohol hangover (1 systematic review)}

The objective of this systematic review ${ }^{20}$ was to assess the clinical evidence on the effectiveness of any medical intervention for preventing or treating alcohol hangover. All randomised controlled trials of any medical intervention for preventing or treating alcohol hangover were included. Trials were considered if they were placebo controlled or controlled against a comparator intervention. Titles and abstracts of identified articles were read and hard copies were obtained. The selection of studies, data extraction, and validation were done independently by two reviewers. The Jadad score was used to evaluate methodological quality. Fifteen potentially relevant trials were identified. Seven publications failed to meet all inclusion criteria. Eight randomised controlled trials assessing eight different interventions were reviewed. The agents tested were propranolol, tropisetron, tolfenamic acid, fructose or glucose, and the dietary supplements Borago officinalis (borage), Cynara scolymus (artichoke), Opuntia ficus-indica (prickly pear), and a yeast based preparation. All studies were double blind. Significant intergroup differences for overall symptom scores and individual symptoms were reported only for tolfenamic acid, $\gamma$ linolenic acid from $B$ officinalis, and a yeast based 
preparation. We concluded that no compelling evidence exists to suggest that any conventional or complementary intervention is effective for preventing or treating alcohol hangover. The most effective way to avoid the symptoms of alcohol induced hangover is to practise abstinence or moderation.

\subsection{Anxiety (3 systematic reviews/1 $\underline{\text { clinical trial) }}$}

\subsubsection{Kava}

The objective of this meta-analysis was to assess the effectiveness and safety as reported in rigorous clinical trials of kava extract compared with placebo for treating anxiety ${ }^{21}$. All publications describing (or which might describe) randomised, double-blind, placebo-controlled trials of kava extract for anxiety were sought through electronic searches on MEDLINE, EMBASE, AMED, CISCOM, Central/CCTR and CCDANCTR. Additionally, manufacturers of kava preparations and experts on the subject were contacted and asked to contribute published and unpublished material. Databases were searched from their respective inception to August 2002. No restrictions regarding the language of publication were imposed. Eleven trials with a total of 645 participants met the inclusion criteria. The meta-analysis of six studies using the total score on the Hamilton Anxiety scale as a common outcome measure suggests a significant reduction in patients receiving kava extract compared with patients receiving placebo (weighted mean difference: $5.0,95 \%$ confidence interval: 1.1 to $8.8 ; \mathrm{p}=0.01 ; \mathrm{n}=345$ ). Analysing a more homogeneous sample reduced the size of this effect (weighted mean difference: $3.4,95 \%$ confidence interval: 0.5 to $6.4 ; \mathrm{p}=0.02 ; \mathrm{n}=305$ ). Adverse events as reported in the reviewed trials were mild, transient and infrequent. We concluded that, compared with placebo, kava extract appears to be an effective symptomatic treatment option for anxiety. The data available from the reviewed studies suggest that kava is relatively safe for shortterm treatment, although more information is required. Further rigorous investigations, particularly into the long-term safety profile of kava are warranted. (This article is also available as a Cochrane Review).

\subsubsection{Autogenic training}

This paper $^{22}$ reports a study to determine the effectiveness of autogenic training in reducing anxiety in nursing students. Nursing is stressful, and nursing students also have the additional pressures and uncertainties shared with all academic students. Autogenic training is a relaxation technique consisting of six mental exercises and is aimed at relieving tension, anger and stress. Meta-analysis has found large effect sizes for autogenic trainings intervention comparisons, medium effect sizes against control groups, and no effects when compared with other psychological therapies. A controlled trial with 50 nursing students found that the number of certified days off sick was reduced by autogenic training compared with no treatment, and a second trial with only 18 students reported greater improvement in Trait Anxiety, but not State Anxiety, compared with untreated controls. A randomized controlled trial with three parallel arms was completed in 1998 with 93 nursing students aged 19-49 years. The setting was a university college in the United Kingdom. The treatment group received eight weekly sessions of autogenic training, the attention control group received eight weekly sessions of laughter therapy, and the time control group received no intervention. The outcome measures were the State-Trait Anxiety Inventory, the Maslach Burnout Inventory, blood pressure and pulse rate completed at baseline, 2 months (end of treatment), and 5, 8, and 11 months from randomization. There was a statistically significantly greater reduction of State $(\mathrm{P}<0.001)$ and Trait $(\mathrm{P}<0.001)$ Anxiety in the autogenic training group than in both other groups immediately after treatment. There were no differences between the groups for the Maslach Burnout Inventory. The autogenic training group also showed statistically significantly greater reduction immediately after 
treatment in systolic $(\mathrm{P}<0.01)$ and diastolic $(\mathrm{P}<0.05)$ blood pressure, and pulse rate $(\mathrm{P}<0.002)$, than the other two groups.

\subsubsection{Herbal remedies}

Anxiety is a prominent indication for herbal medicine. This systematic review ${ }^{23}$ was therefore aimed at summarising the evidence for or against the anxiolytic efficacy of such treatments. Six databases were searched for all randomised clinical trials testing herbal monopreparations in the alleviation of anxiety. Seven such studies and one systematic review were located. Eight different herbals were studied. The herbal medicines, which, according to these data are associated with anxiolytic activity in humans, are Piper methysticum and Bacopa monniera. Only for kava were independent replications available. It was concluded that there is a lack of rigorous studies in this area and that only kava has been shown beyond reasonable doubt to have anxiolytic effects in humans.

\subsubsection{Hypnotherapy}

This systematic review ${ }^{24}$ evaluates the effectiveness evidence from randomised clinical trials (RCTs) for both standalone and adjunctive hypnosis for the treatment of anxiety. Six electronic databases were searched from inception until February 2007. Reference lists of retrieved articles were handsearched. There were no language restrictions. Two reviewers independently identified 14 RCTs of hypnosis for the treatment of anxiety, assessed trial quality, and extracted data. Where possible, between-group analyses were extracted or conducted for this review using software from the Cochrane Collaboration (RevMan 4.2). Methodological heterogeneity of trials precluded metaanalysis. Data were synthesised in a narrative summary. The contribution of many trials was limited by a lack of between-group analyses or because relevant statistical information was omitted. Between-group analyses presented in trial reports, and our own analyses, generally demonstrated no significant differences between hypnosis and control conditions (waiting list controls, contact controls, or other non-standard treatments). It is possible that non-significant findings were a result of small sample sizes. We did find some limited yet consistent evidence that hypnosis may be of benefit in alleviating test anxiety and performance-related social anxiety. The current RCT evidence is insufficient to support the use of hypnosis for treating anxiety. Given the lack of high quality trials in this area, the preliminary evidence of effectiveness for some anxiety types, and the regained popularity of hypnosis, further RCTs are warranted.

\subsection{Arthritis (2 systematic reviews)}

\subsubsection{Nutrition}

Nutritional supplements, such as Seatone, which contain freeze-dried tissue from the New Zealand green-lipped mussel Perna canaliculus, are sold in many countries to relieve arthritic symptoms and to aid in the regeneration of osteo- and rheumatoid arthritic and injured joints. Searches for all published controlled trials on the clinical effectiveness of green-lipped mussel, as a nutritional supplement with potential health benefits for arthritis, were carried out from four independent databases $^{25}$. No language restrictions were imposed, and the review was undertaken from extracted data and was assessed critically according to predefined criteria by the authors. Reports of clinical studies, using freeze dried mussel powder, show mixed outcome measures and are not conclusive, with only two of five randomized controlled trials attesting benefits for rheumatoid and osteoarthritis patients. Similarly, animal studies have likewise yielded mixed findings. In both these cases possibly due to the lack of stabilization of the omega-3 polyunsaturated fatty acids, now known to be the basis of anti-inflammatory activity. There is thus little consistent and compelling evidence, to date, in the 
therapeutic use of freeze-dried green-lipped mussel powder products for rheumatoid or osteoarthritis treatment, particularly in comparison to other cheaper alternative nutriceutical supplements of proven efficacy. However, further investigations are necessary to determine whether green-lipped mussel supplements, such as Seatone, are therapeutic options in the management of arthritis.

\subsubsection{Antioxidants}

Our objective was to systematically review ${ }^{26}$ the evidence from randomized clinical trials (RCTs) for the effectiveness of the antioxidant vitamins A, C, E or selenium or their combination in the treatment of arthritis. A systematic search of computerized databases from inception to September 2006 for relevant RCTs was conducted. Pre-defined inclusion/exclusion criteria were applied and independent data extraction was performed by two authors. Methodological quality was assessed using the Jadad scale. The searches identified 20 unique RCTs meeting the inclusion criteria: 11 in inflammatory arthritis and 9 in osteoarthritis (OA). The studies included are generally of poor quality. They fall into three main clusters: selenium for rheumatoid arthritis $\left(\mathrm{n}^{1 / 45)}\right.$; vitamin $\mathrm{E}$ for inflammatory arthritis $\left(\mathrm{n}^{1 / 45}\right)$ and vitamin E for OA ( $\mathrm{n}^{1 / 47)}$. One RCT suggests superiority of vitamin E over placebo and three RCTs suggest equivalence between vitamin $\mathrm{E}$ and diclofenac in the treatment of inflammatory arthritis. In OA, four RCTs compared vitamin E with placebo. Two shorter-term studies were positive and two longer-term studies were negative. Two further RCTs suggest equivalence between vitamin $\mathrm{E}$ and diclofenac in the treatment of $\mathrm{OA}$. Findings for selenium, vitamin $\mathrm{A}$ and a combination product in inflammatory arthritis and for vitamin $\mathrm{A}$, and a combination product in OA were negative. An isolated positive result for vitamin $\mathrm{C}$ in $\mathrm{OA}$ is of doubtful clinical significance. Conclusions. Clinical trials testing the efficacy of vitamin $\mathrm{E}$ in the treatment of $\mathrm{OA}$ and inflammatory arthritis have been methodologically weak and have produced contradictory findings. There is presently no convincing evidence that selenium, vitamin $\mathrm{A}$, vitamin $\mathrm{C}$ or the combination product selenium ACE is effective in the treatment of any type of arthritis.

\subsection{Asthma (2 surveys/6 systematic reviews/2 clinical trials)}

\subsubsection{Acupuncture}

Contradictory results from randomised controlled trials of acupuncture in asthma suggest both a beneficial and detrimental effect. The authors conducted a formal systematic review and metaanalysis of all randomised clinical trials in the published literature that have compared acupuncture at real and placebo points in asthma patients. ${ }^{27}$ The authors searched for trials published in the period 1970-2000. Trials had to measure at least one of the following objective outcomes: peak expiratory flow rate, forced expiratory volume in one second ( $\left.\mathrm{FEV}_{1}\right)$ and forced vital capacity. Estimates of the standardised mean difference, between acupuncture and placebo were computed for each trial and combined to estimate the overall effect. Heterogeneity was investigated in terms of the characteristics of the individual studies. Twelve trials met the inclusion criteria but data from one could not be obtained. Individual patient data were available in only three. Standardised differences between means ranging from 0.071 to 0.133 , in favour of acupuncture, were obtained. The overall effect was not conventionally significant and it corresponds to an approximate difference in FEV 1 means of 1.7. After exploring heterogeneity, it was found that studies where bronchoconstriction was induced during the experiment showed a conventionally significant effect. This meta-analysis did not find evidence of an effect of acupuncture in reducing asthma. However, the meta-analysis was limited by shortcomings of the individual trials, in terms of sample size, missing information, adjustment of baseline characteristics and a possible bias against acupuncture introduced by the use of placebo points that may not be completely inactive. There was a suggestion of preferential publication of 
trials in favour of acupuncture. There is an obvious need to conduct a full-scale randomised clinical trial addressing these limitations and the prognostic value of the aetiology of the disease.

\subsubsection{Chiropractic}

Some clinicians believe that spinal manipulation is an effective treatment for asthma. The aim of this systematic review ${ }^{28}$ was to critically evaluate the evidence for or against this claim. Four electronic databases were searched without language restrictions from their inceptions to September 2008. Bibliographies and departmental files were hand-searched. The methodological quality of all included studies was assessed with the Jadad score. Only randomised clinical trials of spinal manipulation as a treatment of asthma were included. Three studies met these criteria. All of them were of excellent methodological quality (Jadad score 5) and all used sham-manipulation as the control intervention. None of the studies showed that real manipulation was more effective than sham-manipulation in improving lung function or subjective symptoms. It is concluded that, according to the evidence of the most rigorous studies available to date, spinal manipulation is not an effective treatment for asthma.

\subsubsection{Herbal medicine}

Two surveys ${ }^{6 ; 7}$ had demonstrated the high prevalence of CM usage by UK asthma sufferers. This systematic review was aimed at defining the efficacy of herbal medicines for this condition. ${ }^{29}$ Four computerised literature searches were performed on MEDLINE, Pubmed, Cochrane Library, and EMBASE. Only randomised clinical trials were included. There were no restrictions on the language of publication. The data were extracted in a standardised, pre-defined manner and assessed critically. Seventeen randomised clinical trials were found, six of which concerned the use of traditional Chinese herbal medicine and eight described traditional Indian medicine, of which five investigated Tylophora indica. Three other randomised trials tested a Japanese Kampo medicine, marihuana, and dried ivy leaf extract. Nine of the 17 trials reported a clinically relevant improvement in lung function and/or symptom scores. It was concluded that no definitive evidence for any of the herbal preparations emerged. Considering the popularity of herbal medicine with asthma patients, there is urgent need for stringently designed clinically relevant randomised clinical trials for herbal preparations in the treatment of asthma.

\subsubsection{Breathing techniques}

According to our survey data, ${ }^{6 ; 7}$ breathing techniques are used by an even larger proportion of asthma sufferers. This systematic review was aimed at determining whether or not these interventions are effective. ${ }^{30}$ Four computerised literature searches identified six randomised clinical trials. The results of these studies are not uniform. Collectively the data imply that physiotherapeutic breathing techniques may have some potential in benefiting patients with asthma. The safety issue has so far not been satisfactorily addressed. It was concluded that more studies are required in order to better define the effectiveness of breathing techniques for asthma.

The effects of the Buteyko breathing technique, a device which mimics pranayama (a yoga breathing technique), and a dummy pranayama device on bronchial responsiveness and symptoms were compared over 6 months in a parallel group study. ${ }^{31}$ Ninety patients with asthma taking an inhaled corticosteroid were randomised after a 2 week run in period to Eucapnic Buteyko breathing, use of a Pink City Lung Exerciser (PCLE) to mimic pranayama, or a PCLE placebo device. Subjects practised the techniques at home twice daily for 6 months followed by an optional steroid reduction phase. Primary outcome measures were symptom scores and change in the dose of methacholine provoking a $20 \%$ fall in $\mathrm{FEV}_{1}\left(\mathrm{PD}_{20}\right)$ during the first 6 months. Sixty-nine patients $(78 \%)$ completed the study. 
There was no significant difference in $\mathrm{PD}_{20}$ between the three groups at 3 or 6 months. Symptoms remained relatively stable in the PCLE and placebo groups but were reduced in the Buteyko group. Median change in symptom scores at 6 months was 0 (interquartile range -1 to 1 ) in the placebo group, -1 ( -2 to 0.75 ) in the PCLE group, and -3 ( -4 to 0$)$ in the Buteyko group ( $\mathrm{p}=0.003$ for difference between groups). Bronchodilator use was reduced in the Buteyko group by two puffs/day at 6 months; there was no change in the other two groups $(\mathrm{p}=0.005)$. No difference was seen between the groups in $\mathrm{FEV}_{1}$, exacerbations, or ability to reduce inhaled corticosteroids. It was concluded that the Buteyko breathing technique can improve symptoms and reduce bronchodilator use but does not appear to change bronchial responsiveness or lung function in patients with asthma. No benefit was shown for the Pink City Lung Exerciser.

\subsubsection{Relaxation techniques}

The aim of this systematic review was to determine whether there is any evidence for or against the clinical efficacy of relaxation therapies. ${ }^{32}$ Four independent literature searches were performed on MEDLINE, Cochrane Library, CISCOM and EMBASE. Only randomised clinical trials (RCTs) were included. There were no restrictions on the language of publication. The data from trials that statistically compared the treatment group with that of the control were extracted in a standardised pre-defined manner and critically assessed by two independent reviewers. Fifteen trials were identified, of which nine appropriately compared the treatment group with the control group. Five RCTs tested progressive muscle relaxation or mental and muscular relaxation, two of which showed significant effects of therapy. One RCT investigating hypnotherapy, one of autogenic training, and two of biofeedback techniques revealed no therapeutic effects. Overall, the methodological quality of the studies was poor. We concluded that there is a lack of efficacy for the safety of relaxation therapies in the management of asthma. This deficiency is due to the poor methodology of the studies as well as the inherent problems of conducting such trials. There is some evidence that muscular relaxation improves lung function of patients with asthma but no evidence for any other relaxation technique.

\subsubsection{Homoeopathy}

Homoeopathy is frequently used to treat asthma in children. In the common classical form of homoeopathy, prescriptions are individualised for each patient. There has been no rigorous investigation into this form of treatment for asthma. In a randomised, double-blind, placebocontrolled trial the effects of individualised homoeopathic remedies were compared with placebo medication in 96 children with mild to moderate asthma as an adjunct to conventional treatment. ${ }^{33}$ The main outcome measure was the active quality of living subscale of the Childhood Asthma Questionnaire administered at baseline and follow up at 12 months. Other outcome measures included other subscales of the same questionnaire, peak flow rates, use of medication, symptom scores, days off school, asthma events, global assessment of change, and adverse reactions. There were no clinically relevant or statistically significant changes in the active quality of life score. Other subscales, notably those measuring severity, indicated relative improvements but the sizes of the effects were small. There were no differences between the groups for other measures. This study provides no evidence that adjunctive homoeopathic remedies, as prescribed by experienced homoeopathic practitioners, are superior to placebo in improving the quality of life of children with mild to moderate asthma in addition to conventional treatment in primary care.

\subsection{Allergic rhinitis (1 systematic review)}

The objective of this article ${ }^{34}$ was to systematically evaluate the effectiveness of acupuncture for treating or preventing allergic rhinitis (AR). We retrieved data from 17 electronic databases, 
nonelectronic searches of conference proceedings, our own files of articles, and bibliographies of located articles. All randomized clinical trials (RCTs) of acupuncture for AR were considered for inclusion if they included placebo controls or were controlled against a comparator intervention. One hundred fifteen possibly relevant studies were identified and 12 RCTs met our inclusion criteria. The methodologic quality of the individual trials was variable. Our review includes 7 trials of high quality that met standards of methodologic rigor. All RCTs tested the effectiveness of acupuncture on AR symptoms and none on its curative value. Three RCTs failed to show superiority of acupuncture for treating or preventing symptoms for seasonal AR compared with placebo acupuncture. For perennial AR, 1 study reported favorable effects of acupuncture on a rhinitis symptoms score and 1 found positive results for a nasal symptoms score compared with placebo acupuncture ( $\mathrm{n}_{-} 152$; standard mean difference, $0.45 ; 95 \%$ confidence interval, $0.13-0.78 ; \mathrm{P}_{-} .006$; heterogeneity: $2{ }_{-}{ }_{0} .45, \mathrm{P}$ $\left..50, \mathrm{I} 2 \_0 \%\right)$. Two RCTs compared acupuncture with oral pharmacologic medications. Their results were in favor of acupuncture. In conclusion, the evidence for the effectiveness of acupuncture for the symptomatic treatment or prevention of AR is mixed. The results for seasonal AR failed to show specific effects of acupuncture. For perennial AR, results provide suggestive evidence of the effectiveness of acupuncture.

\subsection{Autism (1 systematic review)}

We aimed to assess the effectiveness of massage as a treatment option for autism. ${ }^{35} \mathrm{We}$ searched the following electronic databases using the time of their inception through March 2010: MEDLINE, AMED, CINAHL, EMBASE, PsycINFO, Health Technology Assessment, Cochrane Central Register of Controlled Trials, Cochrane Database of Systematic Reviews, Database of Abstracts of Reviews of Effects, Psychology and Behavioral Sciences Collection, 6 Korean medical databases (KSI, DBpia, KISTEP, RISS, KoreaMed, and National Digital Library), China Academic Journal (through China National Knowledge Infrastructure), and 3 Japanese medical databases (Journal@rchive, Science Links Japan, and Japan Science \& Technology link). The search phrase used was "(massage OR touch OR acupressure) AND (autistic OR autism OR Asperger's syndrome OR pervasive developmental disorder)." The references in all located articles were also searched. No language restrictions were imposed. Prospective controlled clinical studies of any type of massage therapy for autistic patients were included. Trials in which massage was part of a complex intervention were also included. Case studies, case series, qualitative studies, uncontrolled trials, studies that failed to provide detailed results, and trials that compared one type of massage with another were excluded. All articles were read by 2 independent reviewers (M.S.L. and J-I.K.), who extracted data from the articles according to predefined criteria. Risk of bias was assessed using the Cochrane classification. Of 132 articles, only 6 studies met our inclusion criteria. One randomized clinical trial found that massage plus conventional language therapy was superior to conventional language therapy alone for symptom severity $(\mathrm{P}<.05)$ and communication attitude $(\mathrm{P}<.01)$. Two randomized clinical trials reported a significant benefit of massage for sensory profile $(\mathrm{P}<.01)$, adaptive behaviour $(\mathrm{P}<.05)$, and language and social abilities $(\mathrm{P}<.01)$ as compared with a special education program. The fourth randomized clinical trial showed beneficial effects of massage for social communication $(\mathrm{P}<.05)$. Two nonrandomized controlled clinical trials suggested that massage therapy is effective. However, all of the included trials have high risk of bias. The main limitations of the included studies were small sample sizes, predefined primary outcome measures, inadequate control for nonspecific effects, and a lack of power calculations or adequate follow-up. In conclusions, limited evidence exists for

the effectiveness of massage as a symptomatic treatment of autism. Because the risk of bias was high, firm conclusions cannot be drawn. Future, more rigorous randomized clinical trials seem to be warranted. 


\subsection{Cancer (1 clinical trial/23 reviews)}

\subsection{1 $\underline{\text { Acupuncture }}$}

This systematic review ${ }^{36}$ summarises the existing evidence on acupuncture for cancer-related pain. Literature searches were conducted in seven databases. All clinical studies of acupuncture, electroacupuncture and ear acupuncture in cancer patients with the main outcome measure of pain were included. Data were extracted according to pre-defined criteria by two independent reviewers and methodological quality was assessed using the Jadad scale. Of the seven studies included, one high quality randomised clinical trial of ear acupuncture showed statistically significant pain relief in comparison with placebo ear acupuncture. All the other studies were either non-blinded $(n=2)$ or uncontrolled clinical trials $(n=4)$. Most investigations suffered from methodological flaws such as inadequate study design, poor reporting of results, small sample size and overestimation of the results. We concluded that the notion that acupuncture may be an effective analgesic adjunctive method for cancer patients is not supported by the data currently available from the majority of rigorous clinical trials. Because of its widespread acceptance, appropriately powered RCTs are needed.

The objective of this review ${ }^{37}$ was to assess the effectiveness of acupuncture as a treatment option for hot flashes in patients with breast cancer. We searched the literature using 14 databases from their inceptions to August 2008, without language restrictions. We included randomised clinical trials (RCTs) comparing real with sham acupuncture or another active treatment or no treatment. Their methodological quality was assessed using the modified Jadad score. Three RCTs compared the effects of manual acupuncture with sham acupuncture. One RCT showed favourable effects of acupuncture in reducing hot flash frequency, while other two RCTs failed to do so. The meta-analysis show significant effects of acupuncture compared with sham acupuncture $(\mathrm{n}=189$, weight mean difference, $3.09,95 \%$ confidence intervals -0.04 to $6.23, \mathrm{P}=0.05$ ) but marked heterogeneity was observed in this model ( $\mathrm{v} 2=8.32, \mathrm{P}=0.02, \mathrm{I} 2=76 \%)$. One RCT compared the effects of electroacupuncture (EA) with hormone replacement therapy. Hormone therapy was more effective than EA. Another RCT compared acupuncture with venlafaxine and reported no significant intergroup difference. A further RCT compared acupuncture with applied relaxation and failed to show a significant intergroup difference. In conclusion, the evidence is not convincing to suggest acupuncture is an effective treatment of hot flash in patients with breast cancer. Further research is required to investigate whether there are specific effects of acupuncture for treating hot flash in patients with breast cancer.

The goal of the study ${ }^{38}$ was to assess the effects of acupuncture as a treatment for hot flushes in prostate cancer (PC) patients. The literature was searched using 14 databases with dates ranging from their inceptions to December 2008 and without language restrictions. All clinical studies of any type of acupuncture in PC patients were included. Their main outcome measures had to be vasomotor symptoms. Their methodological quality was assessed using the modified Jadad score. Six studies met all the inclusion criteria. One randomised clinical trial compared the effects of manual acupuncture with acupuncture plus electro-acupuncture. The other five studies were uncontrolled observational studies and therefore had limitations. In conclusion, the evidence is not convincing to suggest acupuncture is an effective treatment for hot flush in patients with PC. Further research is required to investigate whether acupuncture has hot-flush-specific effects.

A systematic review of systematic ${ }^{39}$ reviews found 7 such articles. Most were of good quality but had to rely on poor quality primary studies. Our conclusion was that only for nausea/vomiting is the effectiveness of acupuncture well established. 


\subsubsection{Aromatherapy}

A randomised controlled pilot study was carried out to examine the effects of adjunctive aromatherapy massage on mood, quality of life and physical symptoms in patients with cancer attending a specialist unit. ${ }^{40}$ Participants were randomised to conventional day care alone or day care plus weekly aromatherapy massage using a standardised blend o foils for four weeks. At baseline and at weekly intervals, patients rated their mood, quality of life and the intensity and bother of two symptoms most important to them. Forty-six patients were recruited to the study. Due to a large number of withdrawals, only 11 of 23 (48\%) patients in the aromatherapy group and 18 of 23 (78\%) in the control group completed all four weeks. Mood, physical symptoms and quality of life improved in both groups. There was no statistically significant difference between groups in any of the outcome measures. Despite a lack of measurable benefit, all patients were satisfied with the aromatherapy and wished to continue. Whilst this pilot study has shown that a randomised controlled trial of complementary therapies is feasible, it has also identified several areas that require further consideration when designing future studies, e.g. the recruitment and retention of appropriate numbers of patients and the outcome measures used.

\subsection{3 $\underline{\text { Carctol }}$}

Carctol is a herbal remedy that recently was widely reported to offer a cure for a range of cancers. This article ${ }^{41}$ is aimed at evaluating the evidence that supports this claim. In addition to numerous websites, we conducted systematic searches in six electronic databases. Any factual information relating to Carctol was considered. The websites provided the composition of the herbal mixture and numerous claims as to its effectiveness. However, not a single scientific study of any style was found. The claim that Carctol is of any benefit to cancer patients is not supported by scientific evidence.

\subsubsection{Co-enzyme Q10}

The aim of this systematic review ${ }^{42}$ was to summarize and evaluate the evidence available for oral supplementation with coenzyme Q10 (CoQ10) to improve the tolerability of cancer treatments. Searches for all published and unpublished controlled trials were carried out on seven databases. Manufacturers of CoQ10 were identified and contacted. Controlled clinical trials of monopreparations of CoQ10 administered orally to cancer patients were included. No language restrictions were imposed. Data were extracted independently by two authors according to predefined criteria. Six studies were included in the review, including three randomized clinical trials and three nonrandomized clinical trials. Patients in five of six studies received anthracyclines. The results suggested that CoQ10 provides some protection against cardiotoxicity or liver toxicity during cancer treatment. However, because of inadequate reporting and analysis, as well as questionable validity of outcome measures, the results are not conclusive. Suggestions that CoQ10 might reduce the toxicity of cancer treatments have not been tested by rigorous trials. Further investigations are necessary to determine whether CoQ10 can improve the tolerability of cancer treatments.

\subsubsection{Guided Imagery}

The aim of this systematic review ${ }^{43}$ is to summarise and critically evaluate the evidence available from controlled clinical trials regarding the use of guided imagery as a sole adjuvant therapy for cancer patients. Electronic searches for controlled clinical trials were carried out in eight databases and two clinical trial registers. Trials that featured guided imagery as a sole adjuvant therapy were included. No language restrictions were imposed. Data were extracted and validated independently by two researchers. Six randomised clinical trials were included. Detailed results were available for 
four studies only. Poor reporting and heterogeneous populations, interventions and outcome measures across trials precluded statistical pooling of results. The methodological quality was on average low. Three studies reported significant differences in measures of anxiety, comfort or emotional response to chemotherapy for patients who received guided imagery over the control groups. Two studies showed no differences between guided imagery and other interventions in any of the outcome measures. We concluded that guided imagery, as a sole adjuvant cancer therapy may be psycho-supportive and increase comfort. There is no compelling evidence to suggest positive effects on physical symptoms such as nausea and vomiting. The data seem sufficiently encouraging for the use of guided imagery as an adjuvant cancer therapy to merit further research.

\subsubsection{Green tea}

Tea is one of the most commonly consumed beverages worldwide. Teas from the plant Camellia sinensis can be grouped into green, black and oolong tea. Cross-culturally tea drinking habits vary. Camellia sinensis contains the active ingredient polyphenol, which has a subgroup known as catechins. Catechins are powerful antioxidants. It has been suggested that green tea polyphenol may inhibit cell proliferation and observational studies have suggested that green tea may have cancerpreventative effects. The objectives of this Cochrane review ${ }^{44}$ was to critically assess any associations between green tea consumption and the risk of cancer incidence and mortality. We searched eligible studies up to January 2009 in the Cochrane Central Register of Controlled Trials (CENTRAL), MEDLINE, EMBASE, Amed, CancerLit, Psych INFO and Phytobase and reference lists of previous reviews and included studies. We included all prospective, controlled interventional studies and observational studies, which either assessed the associations between green tea consumption and risk of cancer incidence or that reported on cancer mortality. At least two review authors independently applied the study criteria, extracted data and assessed methodological quality of studies. Due to the nature of included studies, which were mainly epidemiological, results were summarised descriptively according to cancer diagnosis. Fifty-one studies with more than 1.6 million participants were included. Twenty-seven of them were case-control studies, 23 cohort studies and one randomised controlled trial (RCT).

\subsubsection{Homeopathy}

Many cancer patients use homeopathic approaches to increase their body's ability to fight cancer, improve their physical and emotional well-being, and alleviate their pain resulting from the disease or conventional treatments. Homeopathy is highly controversial as there is no plausible mode of action for these highly diluted remedies. The aim of this systematic review ${ }^{45}$ is to summarize and critically evaluate the efficacy of homeopathic remedies used as a sole or additional therapy in cancer care. We have searched the literature using the databases: Amed (from 1985); CINHAL (from 1982); EMBASE (from 1974); Medline (from 1951); and CAMbase (from 1998). Randomised and nonrandomised controlled clinical trials including patients with cancer or past experience of cancer receiving single or combined homeopathic interventions were included. The methodological quality of the trials was assessed by Jadad score. Six studies met our inclusion criteria (five were randomised clinical trials and one was a non-randomised study); but the methodological quality was variable including some high standard studies. Our analysis of published literature on homeopathy found insufficient evidence to support clinical efficacy of homeopathic therapy in cancer care.

\subsubsection{Massage}

Massage is a popular adjunct to cancer palliation. This systematic review ${ }^{46}$ is aimed at critically evaluating all available randomised clinical trials of massage in cancer palliation. Six databases were searched to identify all trials of classical massage for cancer patients. Studies of other types of 
massage, e.g. reflexology, aromatherapy, were excluded. Fourteen trials met all inclusion criteria. Collectively, they suggest that massage can alleviate a wide range of symptoms: pain, nausea, anxiety, depression, anger, stress and fatigue. However, the methodological quality of the included studies was poor, a fact that prevents definitive conclusions. In conclusion, the evidence is, therefore, encouraging but not compelling. The subject seems to warrant further investigations which avoid the limitations of previous studies.

\subsubsection{Moxibustion}

Moxibustion is a traditional Chinese method that uses the heat generated by burning herbal preparations containing Artemisia vulgaris to stimulate acupuncture points. Considering moxibustion is closely related to acupuncture, it seems pertinent to evaluate the effectiveness of moxibustion as a treatment of symptoms of cancer. The objective of this review ${ }^{47}$ was to systematically assess the effectiveness of moxibustion for supportive cancer care. We searched the literature using 11 databases from their inceptions to February 2010, without language restrictions. We included randomised clinical trials (RCTs) in which moxibustion was employed as an adjuvant treatment for conventional medicine in patients with any type of cancer. The selection of studies, data extraction, and validations were performed independently by two reviewers. Five RCTs compared the effects of moxibustion with conventional therapy. Four RCTs failed to show favourable effects of moxibustion for response rate compared with chemotherapy $(\mathrm{n}=229, \mathrm{RR}, 1.04,95 \% \mathrm{CI} 0.94$ to $1.15, \mathrm{P}=0.43)$.

Two RCTs assessed the occurrence of side effects of chemotherapy and showed favourable effects of moxibustion. A meta-analysis showed significant less frequency of nausea and vomiting from chemotherapy for moxibustion group $(\mathrm{n}=80, \mathrm{RR}, 0.38,95 \%$ CIs 0.22 to $0.65, \mathrm{P}=0.0005$, heterogeneity: $\chi 2=0.18, \mathrm{P}=0.67, \mathrm{I} 2=0 \%$ ). In conclusion, the evidence is limited to suggest moxibustion is an effective supportive cancer care in nausea and vomiting. However, all studies have a high risk of bias so effectively there is not enough evidence to draw any conclusion. Further research is required to investigate whether there are specific benefits of moxibustion for supportive cancer care.

\subsubsection{Various treatments}

Unconventional cancer treatments are frequently used by cancer patients. Therefore, oncologists need to know about them. Several reviews were aimed at providing overviews of current knowledge on the most prevalent complementary or alternative cancer therapies. ${ }^{48-50}$ A distinction was made between alleged cures, preventive measures and adjunctive techniques. Shark cartilage, mistletoe, thymus therapy, essiac, hydrazine sulphate, 714-X, dietary regimens, green tea and Panax ginseng were all covered specifically. None of these treatments offer reasonable hope for a cure. Some strategies are promising in terms of cancer prevention. The true potential of unconventional therapies might lie in adjunctive and palliative care. It was concluded that good evidence in this area is scarce. Vis-à-vis the high prevalence of unconventional cancer treatments, rigorous investigations are mandatory, not least for increasing the safety of future patients.

\subsubsection{Palliative care}

Several complementary therapies are particularly relevant to palliative cancer care. Exemplary studies and, where available, systematic reviews are discussed in this overview. ${ }^{51}$ Promising results exist for some treatments, e.g. acupuncture, enzyme therapy, homoeopathy, hypnotherapy, and relaxation techniques. Unfortunately, the evidence is not compelling for any of these therapies. These results point to some potential for complementary medicine in palliative care. They also demonstrate an urgent need for more rigorous research into the value (or otherwise) of such treatments in palliative and supportive cancer care. 


\subsubsection{Herbal medicine}

The aim of this paper was to review the evidence for or against an association between regular consumption of Allium vegetables and cancer. ${ }^{52}$ A MEDLINE search was conducted to identify all publications on the topic. Twenty epidemiological studies were found. The majority investigates the association of onion consumption and cancer, while eight studies assess garlic consumption. With only one exception, these studies suggest that Allium vegetables convey a protective effect, in particular from cancers of the gastrointestinal tract. These results could be due to an artefact but are more likely genuine, as they are based on plausible biological mechanisms. Anti-bacterial or antimutagenic effects of Allium vegetables are prime candidates to explain this relationship. In conclusion, the hypothesis that regular consumption of Allium vegetables reduces the risk of cancer is compelling and would seem to deserve testing in intervention trials.

\subsubsection{Tai chi}

Tai chi is a form of complementary and alternative medicine with similarities to aerobic exercises, which has been recommended for relieving cancer-related symptoms. The aim of this systematic review $^{53}$ is to summarize and critically evaluate the evidence available from controlled clinical trials of tai chi as a supportive therapy for cancer patients. We have searched the literature using 19 databases from their respective inceptions through October 2006, without language restrictions. Methodological quality was assessed using Jadad score. The searches identified 27 potentially relevant studies. Three randomised clinical trials (RCTs) and one non-randomised controlled trial (CCT) met our inclusion criteria. All of these trials assessed patients with breast cancer. Two RCTs reported significant differences in psychological and physiological symptoms compared to psychosocial support control. Most trials suffered from methodological flaws such as small sample size, inadequate study design and poor reporting. The evidence is not convincing enough to suggest that tai chi is an effective supportive treatment for cancer. Further research should attempt to answer the many open questions related to the usefulness of tai chi for supportive cancer care.

The objective of this review ${ }^{54}$ was to assess the effectiveness of tai chi for supportive breast cancer care. Eleven databases were searched from inception through December 2009. Controlled trials testing tai chi in patients with breast cancer that assessed clinical outcome measures were considered. The selection of studies, data extraction, and validations were performed independently by two reviewers. Risk of bias was assessed using Cochrane criteria. Three randomized clinical trials (RCTs) and four non-randomized controlled clinical trials (CCTs) met our inclusion criteria. The three RCTs tested the effects of tai chi on breast cancer care compared with walking exercise, psychological support therapy, or spiritual growth or standard health care and showed no significant differences between tai chi and these control procedures in quality of life and psychological and physical outcome measures. The meta-analysis also failed to demonstrate significant effects of tai chi compared with control interventions $(\mathrm{n}=38, \mathrm{SMD}, 0.45,95 \% \mathrm{CI}-0.25$ to $1.14, \mathrm{P}=0.21$; heterogeneity: $\mathrm{v} 2=0.23, \mathrm{P}=0.63 ; \mathrm{I} 2=0 \%$ ). All of the four CCTs showed favorable effects of tai chi. Three trials suggested effectiveness in psychological and physical outcome measures, whereas one study was too poorly reported to be evaluated in detail. All of the CCTs had a high risk of bias. Collectively, the existing trial evidence does not show convincingly that tai chi is effective for supportive breast cancer care. Future studies should be of high methodological quality, with a particular emphasis on including an adequate control intervention. 


\subsubsection{Thymus therapy}

One mechanism through which some complementary therapies are claimed to decrease malignant tumour load is the stimulation of the immune system. It is hoped that this will reverse immunological deficits and, therefore, delay the progression of the disease. The thymus is thought to be a key organ for the development of cell-mediated immunological defence. Thymic hormones are involved in the maturation of $\mathrm{T}$ lymphocytes. Extracts prepared from this organ are, therefore, promoted for the (adjuvant) treatment of various forms of cancer. A systematic review ${ }^{55}$ evaluated the evidence for and against clinical effectiveness of thymus therapy for cancer. A computerised MEDLINE search (19661996) was conducted (MESH headings: cancer, alternative medicine, thymus extract, cell therapy). In addition, books and non-MEDLINE-listed articles on the subject were searched for references. Several experts in the field were also consulted. All papers thus retrieved were scanned for further relevant references in their bibliographies. Only randomised clinical trials were included in this review. Thirteen such studies were identified. Even though the majority of these trials suggested that thymus therapy is effective, the collective evidence is not compelling. It was concluded that the subject deserves more rigorous research.

\subsubsection{Qigong}

Qigong is a mind-body integrative exercise or intervention from traditional Chinese medicine used to prevent and cure ailments, to improve health and energy levels through regular practice. The aim of this systematic review ${ }^{56}$ is to summarize and critically evaluate the effectiveness of qigong used as a stand-alone or additional therapy in cancer care. We have searched the literature using the following databases from their respective inceptions through November 2006: MEDLINE, AMED, British Nursing Index, CINAHL, EMBASE, PsycInfo, The Cochrane Library 2006, Issue 4, four Korean Medical Databases, Qigong and Energy Medicine Database from Qigong Institute and four Chinese Databases. Randomised and non-randomised clinical trials including patients with cancer or past experience of cancer receiving single or combined qigong interventions were included. All clinical endpoints were considered. The methodological quality of the trials was assessed using the Jadad score. Nine studies met our inclusion criteria (four were randomised trials and five were nonrandomised studies). Eight of these trials tested internal qigong and one trial did not reported details. The methodological quality of these studies varies greatly and was generally poor. All trials related to palliative/supportive cancer care and none to qigong as a curative treatment. Two trials suggested effectiveness in prolonging life of cancer patients and one failed to do so. We conclude that the effectiveness of qigong in cancer care is not yet supported by the evidence from rigorous clinical trials.

\subsubsection{Mistletoe}

An Editorial addressed the question whether mistletoe therapy (which is immensely popular in Continental Europe) benefits cancer patients. ${ }^{57}$ It is easy to find euphemistically positive answers to this question in the recent literature: "some 50 clinical studies report extended survival times, improved quality of life or, in some studies, tumour regression with mistletoe therapy". Such optimistic statements are often based on an analysis which included studies with severe (often fatal) flaws and which is, therefore, far from convincing. Two serious attempts to evaluate mistletoe therapy come to considerably less enthusiastic verdicts. Kleijnen and Knipschild published a systematic review of all 11 controlled clinical trials available in 1994. They found that the average quality of these studies was poor. The majority of these data suggested some benefit, but the only rigorous study turned out to be negative. The authors concluded: "we can not recommend the use of mistletoe extracts in the treatment of cancer patients with an exception for patients involved in clinical trials". More recently, the "Canadian Breast Cancer Research Initiative" assessed the totality 
of the pre-clinical and clinical evidence. The conclusion was similarly reserved: "the evidence of the clinical benefit from human studies remains weak and inconclusive". Now several new trials are emerging. A placebo-controlled trial of Lektinol ${ }^{\circledR}$ (a pure lectin preparation) with 272 patients suffering from breast cancer seems (according to an aggressive advertising campaign by the manufacturer) to show an improvement of quality of life. A further study with melanoma patients seems to have been negative. To judge either of these trials is premature: and we must obviously wait until the full publication of these results become available.

A systematic review was conducted of all randomised clinical trials of mistletoe as a treatment for cancer. ${ }^{58}$ Eight databases were searched to identify all studies which met our inclusion/exclusion criteria. Data were independently validated and extracted by two authors and checked by the third according to predefined criteria. Statistical pooling was not possible because of the heterogeneity of the primary studies. Therefore a narrative systematic review was conducted. Ten trials could be included. Most of the studies had considerable weaknesses in terms of study design, reporting or both. Some of the weaker studies implied benefits of mistletoe extracts, particularly in terms of quality of life. None of the methodologically stronger trials showed efficacy in terms of quality of life, survival or other outcome measures. Rigorous trials of mistletoe extracts fail to demonstrate efficacy of this therapy.

\subsubsection{7 $\underline{\text { Laetrile }}$}

Laetrile is an unconventional therapy which has been used illegally for decades by cancer patients who together with some alternative therapists claim its effectiveness as an anti-cancer treatment. It has been often referred to as amygdalin, although the two are not the same. The aim of this review ${ }^{59}$ was to assess the alleged anti-cancer effect and the possible harms of Laetrile as a sole or adjunctive therapy in cancer treatment. We searched The Cochrane Central Register of Controlled Trials (CENTRAL); MEDLINE (from 1951); EMBASE (from 1980); Allied and Complementary Medicine (AMED), Scirus, CancerLit, CINAHL (all from 1982); CAMbase (from 1998); the MetaRegister; the National Research Register and our own files. No language restrictions were imposed. Randomized clinical trials (RCTs) and non randomized controlled clinical trials (non-RCTs). Two reviewers independently assessed trials for inclusion in the review, assessed study quality and extracted data. No RCTs or non-RCTs were found, so no abstraction of outcome data could be performed in this systematic review. The claim that Laetrile has beneficial effects for cancer patients is not supported by data from controlled clinical trials. This systematic review has clearly identified the need for randomised or controlled clinical trials assessing the effectiveness of Laetrile or amygdalin for cancer treatment.

The aim of this review ${ }^{60}$ is to summarize all types of clinical data related to the effectiveness or safety of laetrile interventions as a treatment of any type of cancer. All types of clinical studies containing original clinical data of laetrile interventions were included. We searched the Cochrane Central Register of Controlled Trials (CENTRAL), MEDLINE (from 1951), EMBASE (from 1980), Allied and Complementary Medicine (AMED), Scirus, CancerLit, Cumulative Index to Nursing and Allied Health (CINAHL; all from 1982), CAMbase (from 1998), the MetaRegister, the National Research Register, and our own files. For reports on the safety of laetrile, we also searched the Uppsala database. No language restrictions were imposed. Thirty six reports met our inclusion criteria. No controlled clinical trials were found. Three articles were nonconsecutive case series, 2 were consecutive case series, 6 were best case series, and 25 were case reports. None of these publications proved the effectiveness of laetrile. Therefore, the claim that laetrile has beneficial effects for cancer patients is not supported by sound clinical data. 


\subsubsection{CAM for breast cancer: recommendations}

Patients diagnosed with breast cancer have many needs that for a start include the expectation of cure. Where cure is unlikely there is always a place for hope and spiritual support. Furthermore whether dealing with the early stages or with the advanced disease patients require symptomatic control that encompasses pain relief, control of nausea and vomiting and psychological distress. To achieve all of these goals there is a need that goes beyond the role of scientific medicine. This position paper ${ }^{61}$ describes the guidelines for the use of complimentary and alternative medicine (CAM) developed by a workshop on behalf of the European Society of Mastology (EUSOMA).

\subsubsection{9 $\underline{\text { Reflexology }}$}

The objective of this review ${ }^{62}$ was to assess the effectiveness of reflexology as a symptomatic treatment for breast cancer. In all, 12 databases were searched from the time of their inception through July 2010. Prospective, controlled clinical trials of reflexology in patients with breast cancer that included an assessment of clinical outcome measures were reviewed. Study selection, data extraction, and validations were performed independently by 2 reviewers. One randomized clinical trial (RCT) and three nonrandomized controlled clinical trials (CCTs) met our inclusion criteria. One large RCT showed significant differences in quality of life and mood when reflexology was compared with self-initiated support. Three CCTs tested reflexology compared with no treatment or simple rest. All of them suggested favorable effects of reflexology on pain, nausea, and vomiting. However, they had a high risk of bias. Collectively, the existing evidence does not convincingly show that reflexology is effective for breast cancer care. Future studies seem warranted; they should be of high methodological quality, and include adequate control interventions.

\subsubsection{CAM for breast cancer: the evidence}

The objectives of this study ${ }^{63}$ was to evaluate and critically analyze all randomized clinical trials (RCTs) of "alternative cancer cures" (ACCs) for breast cancer. The electronic databases Cochrane Central Register of Controlled Trials, MEDLINE, EMBASE, Allied and Complementary Medicine, Scirus, BIOSIS, CancerLit and CINAHL and for ongoing trials the MetaRegister at http://www.controlled-trials.com/ and the National Research Register at http://www.updatesoftware.com/national/ were searched from their inception. Bibliographies of located studies were scanned. Unpublished or ongoing trials were identified through correspondence with experts in the field. Our own files were hand searched for further RCTs. Review methods included a systematic review of RCTs involving breast cancer patients treated with ACCs, survival, parameters indicative of tumor burden, disease progression, cancer recurrence, and cancer cure. Results were tabulated and summarized. Thirteen RCTs met the inclusion criteria. In most cases their methodological quality is low, with only two RCTs scoring "4" and four RCTs scoring "3" out of 5 possible points for methodological quality. The treatments tested included various methods of psychosocial support such as group support therapy, cognitive behavioral therapy cognitive existential group therapy, a combination of muscle relaxation training and guided imagery, the Chinese herbal remedy Shi Quan $\mathrm{Da} \mathrm{Bu}$ Tang, thymus extract, transfer factor, melatonin, and factor AF2. Encouraging but not fully convincing results emerged for melatonin.

\subsection{Carpel Tunnel syndrome (1 systematic review)}

Acupuncture is a widely used symptomatic treatment for carpal tunnel syndrome (CTS). The objective of this systematic review ${ }^{64}$ was to evaluate the evidence of the effectiveness of acupuncture and acupuncture-like treatments for CTS. Systematic searches were conducted on 11 electronic databases without language restrictions. All randomized controlled trials (RCTs) of acupuncture as a 
treatment of CTS were included. Methodological quality was assessed using the Cochrane risk of bias tool. Six RCTs met our inclusion criteria. Their methodological quality was generally low. Two RCTs compared the effectiveness of acupuncture with a sham control. The others used active controls. A meta-analysis of acupuncture versus steroid block therapy favored acupuncture ( 2 studies, $\mathrm{n}=144$; risk ratio, $1.28 ; 95 \% \mathrm{CI}, 1.08$ to $1.52 ; \mathrm{P}=.005$; heterogeneity, $\mathrm{I}(2)=10 \%)$ in terms of responder rate. Our systematic review and meta-analysis demonstrate that the evidence for acupuncture as a symptomatic therapy of CTS is encouraging but not convincing. The total number of included RCTs and their methodological quality were low. Further rigorous studies are required to establish whether acupuncture has therapeutic value for this indication. PERSPECTIVE: This systematic review of RCTs focused on clinical trials testing the effectiveness of acupuncture for CTS. The existing evidence is not convincing enough to suggest that acupuncture is an effective therapy for CTS. Further RCTs should overcome the limitation of previous studies.

\subsection{Chronic obstructive pulmonary disease (1 systematic review)}

The aim of the current study ${ }^{65}$ was to systematically assess the effectiveness of herbal medicines in treating chronic obstructive pulmonary disease (COPD). Randomised clinical trials (RCTs) testing herbal medicines against any type of control intervention in patients with COPD and assessing clinically relevant outcomes were included. The selection of studies, data extraction and validation were performed independently by at least two reviewers. Methodological quality was evaluated using the Jadad score. Effect sizes and their $95 \%$ confidence intervals were calculated. Fourteen eligible RCTs, testing 14 different herbal medicines, were located. Herbal medicines were compared against placebo or no treatment in six trials. Significant intergroup differences for one or more outcome were reported for several herbal medicines including Panax ginseng and Salvia miltiorrhiza. In seven RCTs, which compared herbal medicines with other herbal medicines, the results were mixed. A single trial compared a herbal medicine (Hedera helix leaf extract) with a conventional treatment (ambroxol tablet) and reported no significant difference between groups. Due to the heterogeneity of the data, statistical pooling was not performed. The median methodological quality score was 2 out of a possible maximum 5. The effectiveness of herbal medicines for treating chronic obstructive pulmonary disease is not established beyond reasonable doubt. Currently, the evidence from randomised clinical trials is scarce and often methodologically weak. Considering the popularity of herbal medicine among chronic obstructive pulmonary disease patients, rigorously designed studies seem warranted

\subsection{Congestive heart failure (1 systematic review)}

The aim of this meta-analysis was to assess the evidence from rigorous clinical trials of the use of hawthorn extract to treat patients with chronic heart failure. ${ }^{66} \mathrm{We}$ searched the literature using MEDLINE, EMBASE, the Cochrane Library, CINAHL, CISCOM, and AMED. Experts on, and manufacturers of, commercial preparations containing hawthorn extract were asked to contribute published and unpublished studies. There were no restrictions about the language of publication. Two reviewers independently performed the screening of the studies, selection, validation, data extraction, and the assessment of methodological quality. To be included, studies were required to state that they were randomised, double-blind, and placebo-controlled, and used hawthorn extract monopreparations. Thirteen trials met all inclusion criteria. In most of the studies, hawthorn was used as an adjunct to conventional treatment. Eight trials including 632 patients with chronic heart failure (New York Heart Association classes I to III) provided data that were suitable for meta-analysis. For the physiological outcome of maximum workload, treatment with hawthorn extract was more beneficial than placebo (weighted mean difference, 7 Watt; 95\% confidence interval [CI]: 3 to 11 Watt; $P<0.01 ; \mathrm{n}=310$ patients). The pressure-heart rate product also showed a beneficial decrease (weighted mean difference, - 20;95\% CI: -32 to $-8 ; \mathrm{n}=264$ patients) with hawthorn treatment. 
Symptoms such as dyspnoea and fatigue improved significantly with hawthorn treatment as compared with placebo. Reported adverse events were infrequent, mild, and transient; they included nausea, dizziness, and cardiac and gastrointestinal complaints. In conclusion, these results suggest that there is a significant benefit from hawthorn extract as an adjunctive treatment for chronic heart failure.

This was also published as a Cochrane Review. ${ }^{67}$

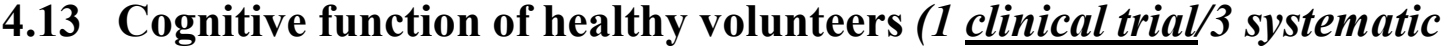 reviews)}

\subsubsection{Ginkgo biloba}

A pilot study was conducted to assess multiple crossover $n=1$ trials with verum/placebo discrimination as the outcome measure as a means of identifying responders and non-responders to the acute nootropic effect of Ginkgo biloba among healthy volunteers. ${ }^{6}$ Multiple double-blind, placebo-controlled $n=1$ trials were performed with 8 treatments in randomised order and separated by minimum washout periods of 7 days. Treatments were acute $120 \mathrm{mg}$ doses of G. biloba extract (GK501) or undistinguishable placebo. The frequency distribution of correct scores for verumplacebo discrimination was compared with the binomial distribution to identify putative responders, who were then tested for consistency of performance over a further 8 treatments. The frequency distribution of scores $(\mathrm{n}=11)$ was bimodal and a discontinuity defined 3 putative responders and 2 putative negative responders for re-test. Two of the putative responders again performed at above chance level and the probability of achieving their scores or better by chance was 0.013 and 0.052 . It was concluded that $\mathrm{n}=1$ trials with verum/placebo discrimination as outcome are a promising method for exploring response heterogeneity to treatments with a subjective effect. Preliminary evidence suggests that there are responders and non-responders to an acute Ginkgo biloba treatment among healthy subjects.

Extracts of Ginkgo biloba are widely used to alleviate or delay the progress of age-related cognitive impairment. Its use as a 'smart' drug by healthy individuals and has also been commercially promoted. The aim of this article was to systematically review and critically evaluate the trial data to test whether Ginkgo biloba enhances cognitive function in healthy subjects. ${ }^{69}$ Literature searches of 6 computerised databases were made for placebo-controlled, double-blind trials of the effect of standardised Ginkgo biloba extracts on cognitive function in healthy subjects. Trials published in any language were included and data were extracted independently by the authors following a standardised protocol. Nine trials were identified, and these were mainly short term. The longest had a treatment period of 30 days. Trials were mostly of good intrinsic methodological quality, but certain aspects of methodology were inadequately reported by all trials. Taken together, these studies indicate no marked or consistent positive effects of Ginkgo biloba on any particular objective measure of cognitive function. A positive subjective effect was reported only in the longest trial. It is concluded that a positive effect of Ginkgo biloba on cognitive function is not proven by data from rigorous clinical trials. The use of Ginkgo biloba as a 'smart' drug cannot be recommended on the basis of the evidence available to date, and there is a particular need for further long-term trials with healthy subjects.

Here, we update our earlier systematic review of 2001, which critically evaluated the data from clinical trials to determine whether Ginkgo biloba enhances cognitive function in healthy subjects. ${ }^{70}$ Literatures searches of six computerised databases, updated to January 2007, were made for randomised, placebo-controlled, double-blind clinical trials of the effects of standardised Ginkgo 
biloba (G. biloba) extracts on cognitive function in healthy subjects under the age of 60 years. Trials published in any language were included, and data were extracted independently by the two authors following a standardised protocol. We include 15 randomised clinical trials of which 7 are singledose studies and 8 are longer term studies with treatment periods ranging from 2 days to 13 weeks. Three single dose studies and 4 longer term studies are newly included. Several of the studies have methodological flaws. A number of the acute studies used multiple outcomes and report positive effects on one or more of these at particular time points with particular doses but these findings are either not replicated, or are directly contradicted by other studies. The evidence from longer term studies is largely negative. Of those studies which measured subjective effects, only one of five acute studies and one of six longer term studies reported any significant positive results. Overall, and in line with our previous conclusions, we have found no convincing evidence from randomised clinical trials for a robust positive effect of G. biloba ingestion upon any aspect of cognitive function in healthy young people, after either acute or longer term administration.

\subsubsection{Transcendental meditation}

It is claimed that regular practice of Transcendental Meditation (TM) improves cognitive function and increases intelligence. This systematic review ${ }^{71}$ assesses the evidence from randomised controlled trials for cumulative effects of TM on cognitive function. Searches were made of electronic databases and the collected papers and official websites of the TM organisation. Only randomised controlled trials with objective outcome measures of the cumulative effects of TM on cognitive function were included. Trials that measured only acute effects of TM, or used only neurophysiological outcome measures were excluded. 107 articles reporting the effects of TM on cognitive function were identified and 10 met the inclusion criteria. Most were excluded because they used no controls or did not randomise subjects between interventions. Of the 10 trials included, 4 reported large positive effects of TM on cognitive function, four were completely negative, and 2 were largely negative in outcome. All 4 positive trials recruited subjects from among people favourably predisposed towards TM, and used passive control procedures. The other 6 trials recruited subjects with no specific interest in TM, and 5 of them used structured control procedures. The association observed between positive outcome, subject selection procedure and control procedure suggests that the large positive effects reported in 4 trials result from an expectation effect. The claim that TM has a specific and cumulative effect on cognitive function is not supported by the evidence from randomised controlled trials.

\subsection{Constipation (2 systematic reviews)}

Abdominal massage therapy was once an accepted treatment for constipation. Subsequently interest faded, but recent years have seen a revival of interest in abdominal massage. It is therefore timely to ask whether or not it is an effective form of treatment for this condition. Computerised literature searches were carried out to retrieve all controlled clinical trials. ${ }^{72}$ Data were extracted in a standardised, pre-defined fashion. Four studies met the inclusion/exclusion criteria. None of the four studies is free of methodological flaws. They are heterogeneous in terms of trial design, patient sample and type of massage used. Nonetheless the results of these trials collectively imply that massage therapy could be a promising treatment for chronic constipation. Future, more rigorous trials should evaluate its true value.

Several studies reported that moxibustion was effective in treating constipation. This systematic review ${ }^{73}$ assesses the clinical evidence for or against moxibustion for treating constipation. Twelve databases were searched from their inception to March 2010. Only randomized clinical trials (RCTs) were included if they compared moxibustion with placebo, sham treatment, drug therapy or no treatment. The methodological quality of these RCTs was assessed with the Cochrane risk of bias 
analysis. All three RCTs included in the study had a high risk of bias. Two included studies found favorable effects of moxibustion. The third RCT showed significant effects in the moxibustion group. Given that the methodological quality of all RCTs was poor, the results from the present review are insufficient to suggest that moxibustion is an effective treatment for constipation. More rigorous studies are warranted.

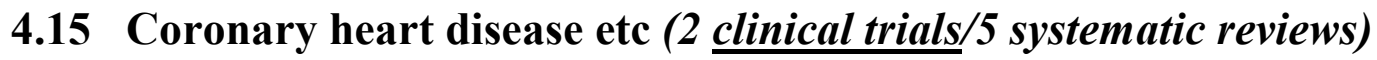

\subsubsection{Acupuncture}

Acupuncture has been reported to affect the autonomic system. Currently, there are no systematic reviews examining the effect of acupuncture on HRV available in the literature. Therefore, the aim of this systematic review ${ }^{74}$ was to summarize and critically assess the effects of acupuncture on heart rate variability. We searched the literature using 14 databases for articles published from the earliest available publications until October 2009 without language restrictions. We included randomized clinical trials (RCTs) comparing acupuncture and sham acupuncture. The risk of bias in each study was assessed using the Cochrane criteria. Twelve RCTs met all of the inclusion criteria. One RCT evaluated the effects of acupuncture in patients with minor depression or anxiety disorders and another RCT examined the effect of acupuncture on migraine patients. Another four RCTs tested the effects of acupuncture in healthy subjects who were exposed to several conditions, including mental stress, fatigue from driving, and caffeine intake. The remaining six RCTs assessed the effects of acupuncture on healthy subjects in a normal state without any stressors. Five RCTs found significant differences in HRV between patients treated with acupuncture versus those treated with sham acupuncture (controls). However, the majority of the other RCTs showed inconsistent results or did not identify significant differences in HRV spectral parameters among individuals treated with acupuncture as compared to those treated with sham acupuncture. In conclusion, sham-controlled RCTs showed variable results and no clear evidence that acupuncture has any specific effects on HRV. Therefore, more rigorous research appears to be warranted.

\subsubsection{Autogenic training}

Autogenic training (AT) is a method of autosuggestion with some potential for reducing anxiety. This study $^{75}$ tests whether AT lowers anxiety levels experienced by patients undergoing coronary angioplasty. Fifty-nine patients were randomly assigned to receive regular AT or no such therapy as an adjunct to standard care for 5 months. The primary outcome measure was State Anxiety at 2 months. Qualitative information was generated by face-to-face interviews. State Anxiety showed a significant intergroup difference both at 2 and 5 months. This finding was corroborated by secondary outcome measures, for example, quality of life, and by qualitative information about patients' experiences. The results do not allow us to determine whether the observed effects are specific to AT or of a non-specific nature. Our results suggest that AT may have a role in reducing anxiety of patients undergoing coronary angioplasty.

The objectives of this study ${ }^{76}$ were to explore autogenic training (AT) as a treatment for psychological morbidity, symptomology, and physiological markers of stress among women with chest pain, a positive exercise test for myocardial ischemia, and normal coronary arteries (cardiac syndrome X). Fifty-three women with cardiac syndrome X (mean T SD age, 57.1 T 8 years) were randomized to an 8-week AT program or symptom diary control. Symptom severity and frequency, Hospital Anxiety and Depression Scale, Spielberger State-Trait Anxiety Inventory, Cardiac Anxiety Questionnaire (CAQ), and Ferrans and Powers Quality of Life Index (QLI), blood pressure, heart rate, electrocardiogram, and plasma catecholamines were measured before and after intervention and 
at the 8-week follow-up. Women who underwent AT had improved symptom frequency $(8.04 \mathrm{~T}$ 10.08 vs $1.66 \mathrm{~T} 2.19$, P G 0.001$)$ compared with control women and reduced symtom severity $(2.08$ $\mathrm{T} 1.03$ vs $1.23 \mathrm{~T} 1.36, \mathrm{P}=0.02)$ and frequency (6.11 T $3.17 \mathrm{vs} 1.66 \mathrm{~T} 2.19, \mathrm{P} \mathrm{G} 0.001)$ post-AT compared with baseline within group. Within-group improvements among women who underwent AT include QLI health functioning (17.80 T 5.74 vs $19.41 \mathrm{~T} 5.19, \mathrm{P}=0.04)$ and CAQ fear $(1.53 \mathrm{~T}$ $0.61 \mathrm{vs} 1.35 \mathrm{~T} 0.56, \mathrm{P}=0.02)$ post-AT and QLI health functioning (17.80 T 5.74 vs $20.09 \mathrm{~T} 5.47, \mathrm{P}$ $=0.01)$, CAQ fear (1.53 T 0.61 vs $1.30 \mathrm{~T} 0.67, \mathrm{P}=0.002)$, CAQ total $(1.42 \mathrm{~T} 0.54$ vs $1.29 \mathrm{~T} 0.475, \mathrm{P}$ $=0.04)$, Spielberger State-Trait Anxiety Inventory trait anxiety (42.95 T 11.19 vs $38.68 \mathrm{~T} 11.47, \mathrm{P}=$ $0.01)$, and QLI quality of life (20.67 T 5.37 vs $21.9 \mathrm{~T} 4.89, \mathrm{P}=0.02)$ at follow-up. In conclusion, an 8 -week AT program improves symptom frequency, with near-significant improvements in symptom severity in women with cardiac syndrome $\mathrm{X}$.

\subsubsection{Chelation therapy}

Chelation therapy is popular in the United States and Europe. The question of whether it does more good than harm remains controversial. The aim of this systematic review ${ }^{77}$ was to summarise all the clinical evidence for or against the effectiveness and efficacy of chelation therapy for coronary heart disease. A thorough search strategy was implemented to retrieve all clinical investigations regardless of whether they were controlled or uncontrolled. The most striking finding is the almost total lack of convincing evidence for its effectiveness. Numerous case reports and case series were found. The majority of these publications seem to indicate that chelation therapy is effective. Only 2 controlled clinical trials were located. They provide no evidence that chelation therapy is efficacious beyond a powerful placebo effect. Given the potential of chelation therapy to cause severe adverse effects, this treatment should now be considered obsolete.

\subsubsection{Herbal medicine}

Many patients use herbal supplements to treat chronic cardiovascular conditions and often combine herbal ingredients with cardiovascular medications. However, physicians do not reliably elicit a history of herbal use from their patients and may overlook herbal supplements' adverse effects. Although often considered harmless by patients, herbal supplements may cause adverse cardiovascular effects from an herbal ingredient, a contaminant, or an herb-drug interaction. Herbal stimulants, including bitter orange, ephedra, caffeine, guarana, maté, kola, areca, lobelia, khat, and others are the most common category of herbal therapies to cause cardiovascular effects. However, dozens of other herbal ingredients have also been linked to adverse cardiovascular events. In addition to listed ingredients, herbal supplements may become contaminated at a number of stages during production. Pesticides, heavy metals, bacteria, and pharmaceutical agents have been detected in herbal supplements. Supposedly "herbal" products that are adulterated with prescription anorectics, antidepressants, diuretics, phosphodiesterase-5 inhibitors along with other medications have been identified throughout Europe, North America, and Asia. All of these adulterants have potential cardiovascular effects. Herbal interactions with a variety of cardiovascular medications may also lead to adverse events. Herbal ingredients may cause pharmacokinetic as well as pharmacodynamic herbdrug interactions. We review clinically relevant patterns of adverse cardiovascular reactions to herbal supplements, and we provide resources and recommendations for practicing cardiologists evaluating patients with suspected herbal adverse effects. ${ }^{78}$

\subsubsection{Music therapy}

It has been suggested that patients recovering from acute myocardial infarction and acute cardiac disease may benefit from music therapy. This review ${ }^{79}$ aims to assess whether adjunctive music therapy is effective in patients suffering from various cardiac conditions. Electronic literature 
searches were performed using Medline, the Cochrane Library, Embase, CISCOM, CINAHL, AMED, the British Nursing Index and PsychINFO. References of identified articles were checked for further potential trials. Randomised clinical trials of adjunctive music therapy that involved patients with cardiac conditions were considered. Music therapy was defined as passively listening to music in addition to standard care. Data on study design, experimental intervention, control intervention, primary outcome parameters, statistics and results were extracted in a standardised manner. To be included, studies had to quantify endpoints relevant for cardiac conditions. Twelve randomised clinical trials were included in the systematic review. Eight of these showed significant benefits in a range of endpoints of music therapy over no such treatment. Music therapy was seen as superior to no treatment in three studies measuring physiological outcomes. In six trials, music therapy was superior to the control condition for psychological outcome measures. In three of the included studies music therapy was not superior to the control condition in any of the outcome measures. Collectively, these data suggest that music therapy shows some promise to lower patient anxiety, heart rate and possibly blood pressure of patients with cardiac conditions. The use of MT for cardiac patients warrants further rigorous investigation.

\subsubsection{Tai chi}

The objective of this systematic review ${ }^{80}$ and meta-analysis was to assess the evidence of tai chi for CVD and its risk factors. Databases searched from their respective inceptions through to March 2007 were: MEDLINE, AMED, British Nursing Index, CINAHL, EMBASE, PsycInfo, the Clinical Trials.gov of the National Institute of Health and National Research Register, Korean Medical Databases, Chinese Academic Journals Databases (CNKI), and the Cochrane Library 2007, issue 1. There were no language restrictions. Prospective, randomized, controlled clinical trials of tai chi for CVD or any of its risk factors were included. Trials comparing tai chi with any type of control group were included. A modified Jadad score was used whereby a point was given for blinding if the outcome assessor was blinded. The mean change in blood pressure compared with baseline was defined as the primary endpoint, and was used to assess the difference between the intervention groups and control groups. The literature searches identified 164 potentially relevant studies of which nine randomized, controlled trials (RCTs) were included (Table 1). One ongoing RCT, which is funded by the National Center for Complementary and Alternative Medicine, tests tai chi for chronic heart failure compared with an education programme.

\subsection{Delayed onset muscle soreness (2 systematic reviews)}

\subsubsection{Homoeopathy}

The question whether or not homoeopathic remedies are more effective than placebos remains unresolved. Delayed-onset muscle soreness (DOMS) is a suitable model to test this hypothesis. Computerised literature searches were conducted to retrieve all placebo-controlled trials on the subject. ${ }^{81}$ Eight such studies were identified. Most trials were burdened with serious methodological flaws. All of the randomised studies showed no significant differences between placebo and verum. It was concluded that the published evidence to date does not support the hypothesis that homoeopathic remedies used in these studies (mainly Rhus toxicodendron and Arnica montana) are more efficacious than placebo in alleviating the symptoms of DOMS.

\subsubsection{Massage therapy}

Massage therapy is often recommended for DOMS but uncertainty exists about its effectiveness. Therefore we wanted to determine whether post-exercise massage alleviates the symptoms of DOMS 
after a bout of strenuous exercise. ${ }^{82}$ Various computerised literature searches located seven controlled trials. Most of the trials were burdened with serious methodological flaws, and their results are far from uniform. However, most suggest that post-exercise massage may alleviate symptoms of DOMS. Thus massage therapy may be a promising treatment for DOMS. Definitive studies are warranted.

\subsection{Dementia (4 systematic reviews)}

\subsubsection{Acupuncture}

Acupuncture is often used as a treatment for dementia and is claimed to be effective in improving intelligence. The objective of this review ${ }^{83}$ is to assess the clinical evidence for or against acupuncture as a treatment for Alzheimer's disease (AD). We searched the literature using 17 databases from their inception to August 2008, without language restrictions. We included all randomised clinical trials (RCTs) of needle acupuncture to treat human patients suffering from AD. Methodological quality was assessed using the Jadad score. Three RCTs met all inclusion criteria. Two RCTs assessed the effectiveness of acupuncture on cognitive function compared with drug therapy. Their results suggested no significant effect in favour of acupuncture $[n=72$, weight mean difference (WMDs), ) 0.55 ; 95\% confidence intervals (CIs) ) 1.31 to $0.21, \mathrm{p}=0.15$, heterogeneity: $\mathrm{s} 2$ $=0, \mathrm{v} 2=0.048, \mathrm{p}=0.49, \mathrm{I} 2=0 \%$ ]. Two RCTs tested acupuncture for activities of daily living (ADL). One RCT reported favourable effects of drug therapy compared with acupuncture for ADL, while the other failed to so. The meta-analysis of these data showed significant effects of drug therapy compared with acupuncture ( $\mathrm{n}=72$, WMD, $) 1.29 ; 95 \%$ CIs: $) 1.77$ to $) 0.80, \mathrm{p}<0.001$, heterogeneity: $\mathrm{s} 2=0, \mathrm{v} 2=0.17, \mathrm{p}=0.68, \mathrm{I} 2=0 \%$ ). Conclusion: Even though the number of studies is small, the existing evidence does not demonstrate the effectiveness of acupuncture for AD.

\subsubsection{Nutrition}

The aim of this systematic review was to determine whether there is cogent evidence in the published literature for a link between dementia and nutrition. ${ }^{84}$ Six case control studies and 5 longitudinal investigations were included. Most of these studies were methodologically flawed. No clear association emerged between nutritional factors and dementia. There were some weak, positive but unconfirmed associations between meat consumption, intake of total fat, saturated fat and cholesterol with dementia incidence. Conversely, regular wine and fish consumption was associated with a decrease of dementia incidence, a finding which was also unconfirmed by independent data. It is concluded that, at present, no firm links have been established between nutritional factors and dementia. The few associations that have been suggested are potentially important and should be investigated further.

\subsubsection{Herbal medicine}

A further article systematically reviewed the clinical evidence of Ginkgo biloba preparations as a symptomatic treatment for dementia. ${ }^{85}$ Computerised literature searches were performed to identify all double-blind, randomised, placebo-controlled trials with clinical endpoints of Ginkgo biloba as a treatment for dementia. Databases included MEDLINE, EMBASE, BIOSIS and the Cochrane Library. There were no restrictions on the language of publication. Data were extracted by both authors in a standardised, pre-defined fashion. Ten such trials were found. They were of varying methodological quality. Although a number of caveats pertain, the studies collectively suggested that Ginkgo biloba is more effective for dementia compared with placebo. Few, generally mild adverse effects have been reported. These findings are encouraging and warrant independent, large-scale confirmatory and comparative trials. 


\subsubsection{Ginseng}

The objective of this review ${ }^{86}$ is to assess the clinical evidence for or against ginseng as a treatment for Alzheimer's disease (AD).We searched 20 databases from their inception to January 2009 and included all randomized clinical trials (RCTs) of any type of Panax ginseng to treat human patients suffering from AD. Methodological quality was assessed using the Jadad score. Two RCTs met all inclusion criteria. They assessed the effectiveness of ginseng as an adjunct to drug therapy on cognitive function compared with conventional drug therapy. Their results suggested significant effect in favor of ginseng on the Mini-Mental Status Examination $(\mathrm{n}=174$, weight mean difference (WMD), 1.85 ; $95 \%$ confidence intervals, CIs 0.88 to $2.82, \mathrm{P}=0.0002$ ) and on the Alzheimer's Disease Assessment Scale (ADAS)-cognitive ( $\mathrm{n}=174$, WMD, 3.09; 95\% CIs 1.08 to 5.09, P $=0.003$ ). Both of these studies are burdened with serious methodological limitations. In conclusion, the evidence for ginseng as a treatment of $\mathrm{AD}$ is scarce and inconclusive. Further rigorous trials seem warranted.

\subsection{Depression (7 systematic reviews)}

\subsubsection{Acupuncture}

The objective of this systematic review ${ }^{87}$ was to summarise the existing evidence on acupuncture as a therapy for depression. RCTs were included, in which either manual acupuncture or electroacupuncture was compared with any control procedure in subjects with depression. Data were extracted independently by two authors. The methodological quality was assessed. Pre and post means and SDs for depression specific measures were extracted, when available, for meta-analysis. Seven randomised comparative trials involving 509 patients were included. The evidence is inconsistent on whether manual acupuncture is superior to sham, and suggests that acupuncture was not superior to waiting list. Evidence suggests that the effect of electroacupuncture may not be significantly different from antidepressant medication, weighted mean difference $-0.43(95 \% \mathrm{CI}-5.61$ to 4.76). There is inconclusive evidence on whether acupuncture has an additive effect when given as an adjunct to antidepressant drugs. We concluded that the evidence from controlled trials is insufficient to conclude whether acupuncture is an effective treatment for depression, but justifies further trials of electroacupuncture.

Acupuncture is often advocated as a treatment for depression, and several trials have tested its effectiveness. Their results are contradictory and even systematic reviews of these data do not arrive at uniform conclusions. The aim of this review ${ }^{88}$ is to critically evaluate all systematic reviews of the subject with a view of assisting clinical decisions. Thirteen electronic databases were searched to identify all relevant articles. Data of these systematic reviews and the primary studies they included were extracted independently by the two authors according to predefined criteria. Eight systematic reviews including seventy-one primary studies were found. Five of the reviews arrived at positive conclusions and three did not. All the positive reviews and most of the positive primary studies originated from China. There are reasons to believe that these reviews are less than reliable. In conclusion, the effectiveness of acupuncture as a treatment of depression remains unproven and the authors' findings are consistent with acupuncture effects in depression being indistinguishable from placebo effects 


\subsubsection{Massage}

This systematic review ${ }^{89}$ evaluates the evidence, from randomised clinical trials (RCTs), for the effectiveness of multiple sessions of classical European (Swedish) massage therapy (MT) for the treatment of depression. Eligible RCTs were identified via eight electronic databases and manual searches of references. Two reviewers independently selected trials, assessed trial quality and extracted data. Four RCTs met our inclusion criteria. Three of these RCTs compared MT with relaxation therapies, but provided insufficient data and analyses to contribute meaningfully to the evaluation of MT for depression. The fourth RCT used MT as a control condition to evaluate a depression-specific acupuncture treatment. This trial provided limited evidence that MT is less effective than acupuncture for treating depression, a treatment which itself is not accepted for this condition. Despite previous research suggesting that MT may be an effective treatment for depression, there is currently a lack of evidence to support this assertion from RCTs that have selected participants for depression.

\subsubsection{Various complementary therapies}

Depression is one of the most common reasons for using complementary and alternative therapies. The aim of this article was to provide an overview of the evidence available on the treatment of depression with complementary therapies. ${ }^{90}$ Systematic literature searches were performed using several databases, reference list searching, and enquiries to colleagues. Data extraction followed a pre-defined protocol. The amount of rigorous scientific data to support the efficacy of complementary therapies in the treatment of depression is extremely limited. The areas with the most evidence for beneficial effects are exercise, herbal therapy (Hypericum perforatum), and, to a lesser extent, acupuncture and relaxation therapies. There is a need for further research involving randomised clinical trials into the efficacy of complementary and alternative therapies in the treatment of depression.

\subsubsection{St John's wort}

The aim of this review was to assess controlled clinical trials of St John's wort (Hypericum perforatum) as a treatment for depression. ${ }^{91}$ A systematic, combined computer and hand search revealed fourteen studies conducted against placebo and four against standard medication. Eight of the former and three of the latter met the predetermined methodological inclusion criteria and were admitted for analysis. The cumulative data show that Hypericum perforatum is superior to placebo in alleviating symptoms of depression as quantified by the Hamilton Depression Scale. It also seems equally effective as standard medication; the frequency of adverse drug reactions shows a clear advantage of the herbal over synthetic anti-depressants. It is concluded that Hypericum perforatum is a safe and effective symptomatic treatment for various forms of depression.

This review ${ }^{92}$ was aimed at providing an updated evaluation of the clinical evidence regarding St John's wort (Hypericum perforatum) as an antidepressant, based on the recently published randomised clinical trials. Computerised literature searches revealed six trials published since an earlier meta-analysis [Linde K et al. Br Med $J$ 1996;313:253-258]. The results of these studies provide further evidence that Hypericum perforatum is superior to placebo in treating mild or moderate depression. However, there is still insufficient evidence to assess the efficacy of Hypericum perforatum in comparison with conventional, particularly modern, antidepressants. Furthermore, there remains a lack of trials assessing long-term effects, other types of depression and different preparations and doses. It was concluded that recent clinical trials strengthen the case for Hypericum perforatum as an antidepressant, but more work is needed to answer the remaining questions. 
A further review ${ }^{93}$ focused on the mechanism of action of St John's wort. Biochemical and animal studies suggest that the phloroglucinol derivative hyperforin is the main active ingredient of St John's wort, and inhibits the synaptosomal uptake of 5-HT, noradrenaline, dopamine, glutamate and GABA. St John's wort has been shown to alleviate symptoms of mild to moderate depression, and seems to offer significant advantages over conventional antidepressants because it is associated with fewer adverse reactions. However, important herb-drug interactions have been described. In view of its efficacy and safety records, St John's wort should be considered for the first-line treatment of mild to moderate depression.

\subsection{Diabetes mellitus (3 systematic reviews)}

\subsubsection{Herbal medicine}

Many diabetics use some form of CAM. Thus it is important to determine which approaches do more good than harm. ${ }^{94}$ This review identified several herbal medicines with a potential for contributing to glycemic control. Even more are recommended in lay books of CAM. Many of these have the potential to do harm, not least through interactions with prescribed antidiabetic drugs. In the interest of diabetic patients, this area should be researched more systematically.

More than 400 plants with suspected glucose-lowering potential are known. In most cases, the evidence for this action is based purely on animal experiments or traditional use. This, however, provides only limited information about its clinical usefulness. Therefore this paper ${ }^{95}$ reviews direct evidence from clinical trials. A systematic literature search identified 22 such papers. The majority reported positive effects. However, much of the data are of poor methodological quality. Only five randomised controlled trials were found, and even these do not convincingly demonstrate the usefulness of the remedies in question. It is concluded that the use of hypoglycemic plant remedies is not supported by results from rigorous clinical trials and requires further investigation.

\subsubsection{Qigong}

The objective of this systematic review ${ }^{96}$ To summarize and critically evaluate the evidence available from clinical trials (CTs) of qigong for patients with type 2 diabetes. We have searched the literature using 15 databases from their respective inceptions through March 2009 without language restrictions. Risk of bias was assessed using Cochrane criteria. Nine CTs, which included three randomized clinical trials (RCTs), one controlled clinical trial (CCT) and five uncontrolled observational studies (UOSs), met our inclusion criteria. Three RCTs compared qigong plus usual care (including drug therapy) with usual care alone. The quality of these RCTs was poor. Their results suggested favorable effects of qigong on glycosylated hemoglobin (HbA1c), $2 \mathrm{~h}$ plasma glucose (2hPG), insulin sensitivity, and blood viscosity. One CCT compared qigong with no treatment and failed to show favorable effects of qigong on fasting plasma glucose (FPG), $2 \mathrm{hPG}$, $\mathrm{HbA1c}$ and insulin sensitivity. All UOSs reported beneficial effects of qigong on FPG or $2 \mathrm{hPG}$. Currently there are few rigorous trials testing the effectiveness of qigong for type 2 diabetes. The studies that are available are of low methodological quality. Collectively this evidence is insufficient to suggest that qigong is an effective treatment for type 2 diabetes. Rigorously designed trials are warranted to answer the many questions that remain open.

\subsubsection{Tai chi}

Databases systematically searched from their respective inceptions through to May 2007 were: MEDLINE, AMED, British Nursing Index, CINAHL, EMBASE, PsycInfo, Korean Medical 
Databases, Chinese Academic Journals Databases, Qigong and Energy Medicine Database and The Cochrane Library 2007, Issue 2. There were no language restrictions. The search terms used were: (tai chi or taiji or shadow boxing) and (diabetes or insulin), or Korean or Chinese language term for tai chi. In addition, the references of all located articles, relevant published book chapters, our departmental files, and proceedings related to tai chi were hand searched for further articles. Several tai chi researchers $(n=6)$ were contacted and asked to contribute any unpublished trials. Prospective controlled clinical studies of tai chi for Type 2 diabetes were included. Dissertations and abstracts were also considered. Case series and case reports and other uncontrolled studies were excluded. A modified Jadad score was used whereby a point was given for blinding if the outcome assessor was blinded. The literature searches identified 471 potentially relevant studies of which five trials, two randomized clinical trials (RCTs) and three non-randomized clinical trials (CCTs) met the inclusion criteria. Four studies reported the change in fasting blood glucose levels (FBG). One RCT found no significant intergroup differences in FBG compared with a sham intervention. One CCT reported superior effects of tai chi on FBG compared with walking or running, while two other CCTs failed to find a difference compared with a self-management programme, or no treatment. Three trials assessed the effectiveness of tai chi on glycated haemoglobin $\left(\mathrm{HbA}_{1 \mathrm{c}}\right)$. One RCT suggested no effectiveness of tai chi on $\mathrm{HbA}_{1 \mathrm{c}}$ compared with sham exercise, and two CCTs also failed to report intergroup differences compared with no treatment, self management, working or running. There was no mention of adverse events in four trials, and one study reported no adverse events in the treatment group. Only a few clinical trials have tested the effectiveness of tai chi in Type 2 diabetes. Our results suggest that there is no convincing evidence that tai chi reduces $\mathrm{FBG}$ and $\mathrm{HbA}_{1 \mathrm{c}}$ in patients with Type 2 diabetes. For other variables, such as insulin resistance and quality of life, there is also no evidence that tai chi may be helpful. In conclusion, evidence supporting the practice of tai chi for treating Type 2 diabetes is scarce and not convincing. The value of tai chi for this indication therefore remains unproven. ${ }^{97}$

\subsection{Dry eye (1 systematic review)}

The objective of this review ${ }^{98}$ was to assess the effectiveness of acupuncture as a treatment option for treating the condition of dry eye. We searched the literature using 14 databases from their inceptions to 3 December 2009, without language restrictions. We included randomized clinical trials (RCTs) comparing acupuncture with conventional treatment. Their risk of bias was assessed using Cochrane criteria. Results: Six RCTs met all the inclusion criteria. Three RCTs compared the effects of acupuncture with artificial tears in patients with xerophthalmia or Sjögren syndrome. A meta-analysis of these data showed that acupuncture improved tear break-up times $(p<0.0001)$, Schirmer test scores $(\mathrm{p}<0.00001)$, response rates $(\mathrm{p}=0.002)$ and the region of cornea fluorescent staining $(\mathrm{p}=$ 0.0001) significantly more than artificial tears did. The other three RCTs compared the effects of acupuncture plus artificial tears with artificial tears alone. Two of these studies failed to show significant effects of acupuncture, while one reported significant effects. For Schirmer test scores and frequency of artificial tear usage, two RCTs reported superior effects of acupuncture plus artificial tears, while one RCT failed to do so. In conclusion, these results provide limited evidence for the effectiveness of acupuncture for treating dry eye. However, the total number of RCTs, the total sample size and the methodological quality were too low to draw firm conclusions.

\subsection{Eczema (2 systematic reviews, 2 clinical trials)}

\subsubsection{Chinese herbal medicine}

Chinese herbal treatments are being promoted as a treatment for eczema. The aim of this article ${ }^{99}$ was to systematically review the evidence for or against this notion. Extensive literature searches were 
carried out to identify all randomised clinical trials on the subject. Data were extracted from these in a pre-defined standardised fashion. Only two randomised clinical trials were located. Both imply that a complex mixture of Chinese herbs is more effective than placebo in treating eczema. Yet several caveats exist, most importantly the lack of independent replication. Adverse effects have also been reported. At present it is therefore unclear whether Chinese herbal treatments of eczema do more good than harm.

\subsubsection{Any complementary therapy}

The objective of this article was to provide a brief but critical overview of the evidence relating to complementary/alternative medicine (CAM) use, and to offer valid and useful information for dermatologists in clinical practice ${ }^{100}$. Systematic literature searches were conducted on MEDLINE, EMBASE, The Cochrane Library, CISCOM and AMED (until October 2000). Where appropriate, the evaluation of the published literature was based on systematic reviews and randomised controlled trials. After scanning the literature it was decided to focus on a selection of two conditions (atopic dermatitis and chronic venous insufficiency) and two treatment modalities (aloe vera gel and tea tree oil). Data for the life-time prevalence of CAM use by patients with dermatological disease ranges between $35 \%$ and $69 \%$. The most popular modalities include herbalism and (other) dietary supplements, while atopic dermatitis is one of the conditions most frequently treated with CAM. For patients with atopic dermatitis, the evidence relates to autogenic training, hypnotherapy, diet, herbal medicine, and dietary supplements. Compelling evidence of effectiveness exists for none of these therapies. However, some promising data have been reported for those with a psychological component: autogenic training, biofeedback and hypnotherapy. For chronic venous insufficiency, there is relatively convincing evidence for the effectiveness of oral horse chestnut seed extract. The data for aloe vera gel and tea tree oil indicate that for neither is there compelling evidence of effectiveness. The use of CAM treatments is not free of direct and indirect risks, which must be considered.

\subsubsection{Healing}

Johrei is a form of spiritual healing comprising "energy channelling" and light massage given either by a trained healer or, after some basic training, by anyone. This pilot trial ${ }^{101}$ aimed to identify any potential benefits of family-based Johrei practice in childhood eczema and for general health and to establish the feasibility of a subsequent randomised controlled trial. Volunteer families of 3-5 individuals, including at least one child with eczema were recruited to an uncontrolled pilot trial lasting 12 months. Parents were trained in Johrei healing and then practised at home with their family. Participants kept diaries and provided questionnaire data at baseline, 3,6 and 12 months. Eczema symptoms were scored at the same intervals. Scepticism about Johrei is presently an obstacle to recruitment and retention of a representative sample in a clinical trial, and to its potential use in general practice. The frequency and quality of practise at home by families may be insufficient to bring about the putative health benefits. Initial improvements in eczema symptoms and diary recorded illness, could not be separated from seasonal factors and other potential confounders. There were no improvements on other outcomes measuring general health and psychological wellbeing of family members.

\subsubsection{Autologous blood therapy}

Autologous blood therapy (ABT) is used for treating atopic dermatitis (AD) in some European countries and is promoted on internet sites for this condition. However, there is little evidence from rigorous clinical trials to suggest that it is effective. This study aimed to test the effectiveness of ABT for the symptomatic treatment of patients with $\mathrm{AD} .{ }^{102}$ Fifty subjects responded to press 
advertisements, and 31 were randomized within strata of severity at recruitment. Patients were included in a double-blind, placebo-controlled trial and received ABT or placebo once weekly for 5 weeks. Assessments were performed at baseline, at weekly intervals and after a 5-week follow up. The Six Area, Six Sign AD (SASSAD) severity index was pre-defined as the primary outcome measure. The Dermatology Life Quality Index and patient ratings of pruritus, quality of sleep and skin appearance on 100-mm visual analogue scales were defined as secondary outcome measures. Success of patient blinding and adverse events were assessed. Data were analysed on an intention-totreat basis. Analysis of covariance suggested a significant differential change of the SASSAD score between baseline and the end of the follow-up period in favour of ABT. The mean reduction in SASSAD score was 13.5 points (95\% confidence interval, CI 6.6-20.4, $P<0.001)$ over and above placebo; the corresponding value at the end of treatment was $9.6(95 \% \mathrm{CI} 4.2-14.9, P=0.001)$. No clear significant intergroup differences in any of the secondary outcome measures were found. Six patients in the ABT group and 7 in the placebo group reported minor and transient adverse events. These data suggest that, according to the SASSAD score, ABT has beneficial effects in the treatment of $\mathrm{AD}$, although this was not confirmed by the patient-rated assessments. The improvement in observer-rated skin condition suggested by this study needs confirmation in larger trials.

\subsection{Endoscopy (1 systematic review)}

Acupuncture is often used to ease the discomfort during gastrointestinal endoscopy. Our systematic review ${ }^{103}$ found 6 RCTs involving a total of 512 patients. The quality of these trials was variable but some were rigorous. The collective evidence suggests that acupuncture is effective as a supportive intervention for endoscopy. It outperformed sham acupuncture and its effects were similar to those of conventional pre-medication.

\subsection{Erectile Dysfunction (3 systematic reviews)}

\subsubsection{Acupuncture}

We evaluated the current evidence for the use of acupuncture to treat erectile dysfunction (ED). Systematic searches were conducted in 15 electronic databases, with no language restrictions. Handsearches included conference proceedings and our files. a treatment for ED were considered for inclusion, and their methodological quality was assessed using the Jadad score. Of the four studies included, one randomized controlled trial (RCT) showed beneficial effects of acupuncture compared with sham acupuncture in terms of response rate, while another RCT found no effects of acupuncture. The remaining two studies were uncontrolled clinical trials. Collectively these data showed that RCTs of acupuncture for ED are feasible but scarce. Most investigations had methodological flaws, e.g. inadequate study design, poor reporting of results, small sample size, and publication without appropriate peer review process. The evidence is insufficient to suggest that acupuncture is an effective intervention for treating ED. Further research is required to investigate whether there are specific benefits of acupuncture for men with ED. ${ }^{104}$

\subsubsection{Maca}

Maca (Lepidium meyenii) is an Andean plant of the brassica (mustard) family. Preparations from maca root have been reported to improve sexual function. The aim of this review ${ }^{105}$ was to assess the clinical evidence for or against the effectiveness of the maca plant as a treatment for sexual dysfunction. We searched 17 databases from their inception to April 2010 and included all randomised clinical trials (RCTs) of any type of maca compared to a placebo for the treatment of healthy people or human patients with sexual dysfunction. The risk of bias for each study was 
assessed using Cochrane criteria, and statistical pooling of data was performed where possible. The selection of studies, data extraction, and validations were performed independently by two authors. Discrepancies were resolved through discussion by the two authors. Four RCTs met all the inclusion criteria. Two RCTs suggested a significant positive effect of maca on sexual dysfunction or sexual desire in healthy menopausal women or healthy adult men, respectively, while the other RCT failed to show any effects in healthy cyclists. The further RCT assessed the effects of maca in patients with erectile dysfunction using the International Index of Erectile Dysfunction-5 and showed significant effects. In conclusion, The results of our systematic review provide limited evidence for the effectiveness of maca in improving sexual function. However, the total number of trials, the total sample size, and the average methodological quality of the primary studies were too limited to draw firm conclusions. More rigorous studies are warranted.

\subsubsection{Red Ginseng}

Korean red ginseng (unskinned Panax ginseng before it is steamed or otherwise heated and subsequently dried) is one of the most widely used herbal remedies. This systematic review ${ }^{106}$ evaluates the current evidence for the effectiveness of red ginseng for treating erectile dysfunction. Systematic searches were conducted on 20 electronic databases without language restrictions. Hand-

searches included conference proceedings and our files. All randomized clinical studies (RCT) of red ginseng as a treatment of erectile dysfunction were considered for inclusion. Methodological quality was assessed using the Jadad score. Seven RCTs met all the inclusion criteria. Their methodological quality was low on average. Six of the included RCTs compared the therapeutic efficacy of red ginseng with placebo. The meta-analysis of these data showed a significant effect $(\mathrm{n}=349$, risk ratio, $2.40 ; 95 \%$ CI of 1.65, 3.51, $\mathrm{p}<0.00001$, heterogeneity: tau2 $=0.05, \mathrm{c} 2=6.42, \mathrm{p}=0.27, \mathrm{I} 2=22 \%$ ). Subgroup analyses also showed beneficial effects of red ginseng in psychogenic erectile dysfunction $(\mathrm{n}=135$, risk ratio, $2.05 ; 95 \% \mathrm{CI}$ of $1.33,3.16, \mathrm{p}=0.001$, heterogeneity: $\mathrm{c} 2=0.08, \mathrm{p}=0.96, \mathrm{I} 2=$ $0 \%$ ). Collectively these RCTs provide suggestive evidence for the effectiveness of red ginseng in the treatment of erectile dysfunction. However, the total number of RCTs included in the analysis, the total sample size and the methodological quality of the primary studies were too low to draw definitive conclusions. Thus more rigorous studies are necessary.

\subsection{Fatigue (1 clinical trial)}

Fatigue is a common reason for consulting a doctor but there is no definitive treatment. St John's wort has been shown to reduce symptoms of fatigue in depressed patients. It therefore may have potential value as a remedy for fatigue of unexplained origin. This pilot study ${ }^{107}$ aimed to investigate the effect of St John's wort on fatigue in a small group of patients in order to formulate a hypothesis upon which a randomised clinical trial could be subsequently based. The study protocol followed an uncontrolled, open design. Twenty patients consulting their doctors and complaining of fatigue were treated with St John's wort extract (3 x 1 tablet daily) for six weeks. Compared with baseline values, perceived fatigue was significantly lower after 2 weeks of treatment and reduced significantly further after 6 weeks. Symptoms of depression and anxiety were also reduced. Baseline scores suggested that nearly half the sample might have been depressed at the start of the trial which was possibly related to fatigue. These results suggest there is scope for conducting a randomised, placebo-controlled trial to investigate the specific effect of St John's wort on fatigue and that the study design must take account of the role of depression in fatigue.

\subsection{Fibromyalgia (4 systematic reviews)}




\subsubsection{Acupuncture}

Acupuncture is often used and frequently advocated for the symptomatic treatment of fibromyalgia. A systematic review ${ }^{108}$ has previously demonstrated encouraging findings. As it is now outdated, we wanted to update it. We searched seven electronic databases for relevant randomized clinical trials (RCTs). The data were extracted and validated independently by both authors. As no meta-analysis seemed possible, the results were evaluated in narrative form. Five RCTs met our inclusion criteria, all of which used acupuncture as an adjunct to conventional treatments. Their methodological quality was mixed and frequently low. Three RCTs suggested positive but mostly short-lived effects and two yielded negative results. There was no significant difference between the quality of the negative and the positive RCTs. All positive RCTs used electro-acupuncture. The notion that acupuncture is an effective symptomatic treatment for fibromyaligia is not supported by the results from rigorous clinical trials. On the basis of this evidence, acupuncture cannot be recommended for fibromyalgia.

\subsubsection{Chiropractic}

Many patients use chiropractic as a treatment of fibromyalgia, and many chiropractors seem to be convinced that it is effective for that condition. The aim of the study ${ }^{109}$ was to conduct a systematic review of randomised clinical trials testing the effectiveness of chiropractic care for fibromyalgia. Six electronic literature searches were conducted. No language restrictions were applied. Data extraction and validation were carried out by two independent reviewers. Three studies met the inclusion criteria. Their methodological quality was poor. They generated no evidence to suggest that chiropractic care is effective for fibromyalgia. Currently, there is insufficient evidence to conclude that chiropractic is an effective treatment for fibromyalgia.

\subsubsection{Herbal medicine}

We critically evaluated the evidence regarding complementary and alternative medicines (CAMs) taken orally or applied topically for the treatment of FM. ${ }^{110}$ Randomized controlled trials of FM using CAMs, in comparison with other treatments or placebo, published in English up to March 2009, were eligible for inclusion. They were identified using systematic searches of bibliographic databases and manual searching of reference lists. Information was extracted on outcomes, and statistical significance, in comparison with alternative treatment or placebo, and side effects were reported. The methodological quality of the primary studies was determined. Single studies on four CAMs, and three on different approaches to homeopathic care were identified. Their methodological quality was moderate. The homeopathy studies were small, but each reported an improvement in pain. The effects of anthocyanidins, capsaicin and S-adenosylmethionine each showed at least one statistically significant improved outcome compared with placebo. However, the studies of anthocyanidins and capsaicin only demonstrated an improvement in a single outcome, sleep disturbance and tenderness, respectively, of several outcomes considered. No evidence of efficacy was found regarding Soy in a single study. Most of these CAMs were free of major adverse effects and usually associated with only minor adverse effects such as dizziness, nausea and stomach upsets. In conclusion, there is insufficient evidence on any CAM, taken orally or applied topically, for FM. The small number of positive studies lack replication. Further high-quality trials are necessary to determine whether these initial findings can be supported by a larger evidence base.

\subsubsection{Homeopathy}

Homoeopathy is often advocated for fibromyalgia (FM) and many FM patients use it. To critically evaluate all randomised clinical trials (RCTs) of homoeopathy as a treatment for FM, six electronic databases were searched to identify all relevant studies. ${ }^{111}$ Data extraction and the assessment of the 
methodological quality of all included studies were done by two independent reviewers. Four RCTs were found, including two feasibility studies. Three studies were placebo-controlled. None of the trials was without serious flaws. Invariably, their results suggested that homoeopathy was better than the control interventions in alleviating the symptoms of FM. Independent replications are missing. Even though all RCTs suggested results that favour homoeopathy, important caveats exist. Therefore, the effectiveness of homoeopathy as a symptomatic treatment for FM remains unproven.

\subsection{Headaches (5 systematic reviews/2 clinical trials)}

\subsection{1 $\underline{\text { Acupuncture }}$}

The objective of a systematic review ${ }^{112}$ was to assess whether there is evidence that acupuncture is effective in the treatment of recurrent headaches. All randomised or quasi-randomised clinical trials comparing acupuncture with any type of control intervention for the treatment of recurrent headaches were included. Information on patients, interventions, methods, and results was extracted by at least two independent reviewers. A pooled estimate of the responder rate ratio (responder rate in treatment group/responder rate in control group) was calculated as a crude indicator of trial results since metaanalysis of more specific outcome measures was impossible due to heterogeneity and insufficient reporting. Twenty-two trials, including a total of 1042 patients (median 36, range 10 to 150), met the inclusion criteria. Fifteen trials were in migraine patients, six in tension-type headache patients, and in one trial patients with various headaches were included. The majority of the 14 trials comparing true and sham acupuncture showed at least a trend in favour of true acupuncture. The pooled responder rate ratio was 1.53 (95\% confidence interval 1.11 to 2.11 ). The eight trials comparing acupuncture with other treatment forms had contradictory results. The existing evidence therefore suggests that acupuncture has a role in the treatment of recurrent headaches. However, the quality and amount of evidence are not fully convincing.

Tension headache is common, and treatment with acupuncture is frequently recommended, although the evidence of its effectiveness is contradictory. This randomised clinical trial ${ }^{113}$ was designed as a pilot to test procedures, including a novel sham, in preparation for a multi-centre trial investigating the effect of acupuncture as a treatment for tension headache. Ten patients suffering from episodic, tension-type headache were recruited through local newspaper articles. Patients were randomised to receive either brief needling to tender areas or selected traditional points (Group A), or pressure from a cocktail stick supported within a guide tube to defined, non-tender and non-acupuncture areas (Group B). The patients' view of treatment sites was obstructed so that no indication could be gained as to which form of treatment was being given, hence the study was patient-blind. Throughout the period of the trial, duration, frequency and intensity of headaches were recorded, from which the mean weekly headache index was calculated. There was no difference between the changes in weekly headache index in the two groups, comparing scores before and after treatment. However, Group A experienced a considerably larger number of headache-free weeks than Group B. The credibility of the two procedures was tested using a standard credibility questionnaire and a "final verdict". One subject in Group B concluded that she had not received genuine acupuncture, but overall there was no statistical difference between the credibility of treatment in the two groups.

The follow-up of this pilot was published recently. ${ }^{114}$ A multicentre, randomised clinical trial tested the hypothesis that acupuncture is more efficacious than sham control procedure in the prevention of episodic tension-type headache. Fifty subjects were randomised to receive a course of treatment with either brief acupuncture or a sham procedure. Subjects were followed up for 3 months. Changes in headache were assessed by daily diary, the primary outcome measure being the number of days with headache. No significant differences were found between the changes in the two groups for any 
measure at any time point. Results also show that patient blinding was successful. In conclusion, this study does not provide evidence that this form of acupuncture is effective in the prevention of episodic tension-type headache.

\subsubsection{Autogenic training}

This systematic review ${ }^{115}$ was aimed at determining from the published evidence whether autogenic training as sole therapy is effective for prevention of tension-type headaches in adults. Literature searches were performed in January 2005 in six major databases, specifically Medline, EMBASE, AMED, CENTRAL, PsychInfo and CINAHL and information was extracted and evaluated in a predefined manner. Seven controlled clinical trials were included in the review. The methodological quality of these studies was low. Patient samples were generally representative of the more severely affected cases. None of the studies show autogenic training to be convincingly superior to other interventions care. Some trials suggested that the effect of autogenic training is no different from hypnosis and inferior to biofeedback. In conclusion, there is no consistent evidence to suggest that autogenic training is superior to other interventions for prevention of tension headaches, or different from other forms of relaxation. Further studies should investigate the use of standard autogenic training in patients with moderate headache.

\subsubsection{Herbal medicine}

Feverfew (Tanacetum parthenium L.) is a popular herbal remedy for migraine. The objectives of this article were to systematically review the evidence for or against the efficacy of feverfew versus placebo for the prevention of migraine. ${ }^{116}$ Electronic literature searches were performed using the CISCOM (Research Council for Complementary Medicine, London, UK), MEDLINE, EMBASE, BIOSIS and the Cochrane Library databases (each from its inception to April 1998). Manufacturers were contacted and the bibliographies of identified articles checked for further trials. All randomised, placebo-controlled, double-blind trials assessing the efficacy of feverfew for preventing migraine were included. No restrictions regarding the language of publication were imposed. Data on patients, interventions, methods, outcomes and results were extracted in a pre-defined, standardised manner. Methodological quality was evaluated using the scoring system developed by Jadad and colleagues. Both data extraction and the assessment of methodological quality were performed independently by two reviewers. Four trials met the inclusion criteria. The majority of these trials suggested beneficial effects of feverfew compared with placebo. However, the trial with the highest methodological quality, which was also among the largest, found no significant difference between feverfew and placebo. It was concluded that the efficacy of feverfew for the prevention of migraine has not been established beyond reasonable doubt.

\subsubsection{Homoeopathy}

Homoeopathy is often advocated as a prophylaxis of migraine and headaches. The aim of this systematic review ${ }^{117}$ was to evaluate the clinical trials, testing the efficacy of homoeopathy for these indications. Independent computerised literature searches were carried out in 4 databases. Only randomised, placebo-controlled trials were included. Four such studies were found. Their methodological quality was variable but, on average, satisfactory. One study suggested that homeopathic remedies were effective. The other, methodologically stronger trials did not support this notion. It was therefore concluded that the trial data available to date do not suggest that homoeopathy is effective in the prophylaxis of migraine or headache beyond a placebo effect. 


\subsubsection{Spinal manipulation}

The aim of this systematic review was to evaluate the literature examining the effectiveness of spinal manipulation for the treatment of headache disorders. ${ }^{118}$ Computerised literature searches were carried out in MEDLINE, EMBASE, AMED and CISCOM. Studies were included only if they were randomised trials of (any type of) spinal manipulation for (any type of) headache in human patients in which spinal manipulation was compared either to no treatment, usual medical care, a 'sham' intervention, or to some other active treatment. Two investigators independently extracted data on study design, sample size and characteristics, type of intervention, type of control/comparison, direction and nature of the outcome(s). Methodological quality of the trials was also assessed using the Jadad scale. Eight trials were identified that met our inclusion criteria. Three examined tensiontype headaches, three migraines, one 'cervicogenic' headache, and one 'spondylogenic' chronic headache. In two studies, patients receiving spinal manipulation showed comparable improvements in migraine and tension headaches compared to drug treatment. In the 4 studies employing some 'sham' interventions (e.g. laser light therapy), results were less conclusive with 2 studies showing a benefit for manipulation and 2 studies failing to find such an effect. Considerable methodological limitations were observed in most trials, the principal one being inadequate control for non-specific (placebo) effects. Despite claims that spinal manipulation is an effective treatment for headache, the data available to date do not support such definitive conclusions. It is unclear to what extent the observed treatment effects can be explained by manipulation or by non-specific factors (e.g. of personal attention, patient expectation). Whether manipulation produces any long-term changes in these conditions is also uncertain. Future studies should address these two crucial questions and overcome the methodological limitations of previous trials.

\subsection{HIV/AIDS (1 systematic review)}

The use of complementary and alternative medicine (CAM) by patients with HIV/AIDS is widespread. Yet, little is known about the evidence supporting its use for this indication. We conducted a systematic review ${ }^{119}$ of randomized clinical trials assessing the effectiveness of complementary therapies for HIV and HIV-related symptoms. Comprehensive literature searches were performed of seven electronic databases. Data were abstracted independently by two reviewers. Thirty trials met our predefined inclusion/exclusion criteria: 18 trials were of stress management; five of Natural Health Products; four of massage/therapeutic touch; one of acupuncture; two of homeopathy. The trials were published between 1989 and 2003. Most trials were small and of limited methodological rigour. The results suggest that stress management may prove to be an effective way to increase the quality of life. For all other treatments, data are insufficient for demonstrating effectiveness. Despite the widespread use of CAM by people living with HIV/AIDS, the effectiveness of these therapies has not been established. Vis à vis CAM's popularity, the paucity of clinical trials and their low methodological quality are concerning.

\subsection{Hypercholesterolaemia (3 systematic reviews)}

\subsubsection{Garlic}

The purpose of this systematic review and meta-analysis ${ }^{120}$ was to investigate the effect of garlic on total cholesterol level in people with elevated levels. Systematic literature searches were conducted on the MEDLINE, EMBASE, BIOSIS, Cochrane Library, AMED, and CISCOM databases.

Manufacturers of commercial garlic preparations and experts in the field were asked about published or unpublished trials. Selected trials were required to state that they were randomised, double-blind, and placebo-controlled; use garlic monopreparations; include people with mean total cholesterol 
levels of at least $5.17 \mathrm{mmol} / \mathrm{L}(200 \mathrm{mg} / \mathrm{dL})$; and report total cholesterol level as an end point. There were no language restrictions. Two reviewers, blinded to key identifiers of each paper, independently extracted data in a standardised manner and assessed methodological quality by using the Jadad scale. Discrepancies were settled through discussion. In the 13 trials included in the meta-analysis, garlic reduced total cholesterol levels from baseline significantly more than placebo $(P<0.01)$; the weighted mean difference was $-0.41 \mathrm{mmol} / \mathrm{L}(95 \% \mathrm{Cl},-0.66$ to $-0.15 \mathrm{mmol} / \mathrm{L})(-15.7 \mathrm{mg} / \mathrm{dL}$ [Cl, 25.6 to $-5.7 \mathrm{mg} / \mathrm{dL}])$. Six diet-controlled trials with the highest scores for methodological quality revealed a non-significant difference between garlic and placebo groups; the weighted mean difference was $-0.11 \mathrm{mmol} / \mathrm{L}(\mathrm{Cl},-0.30$ to $0.08 \mathrm{mmol} / \mathrm{L})(-4.3 \mathrm{mg} / \mathrm{dL}$ [Cl, -11.7 to $3.1 \mathrm{mg} / \mathrm{dL}])$. It was concluded that the available data suggest that garlic is superior to placebo in reducing total cholesterol levels. However, the size of the effect is modest, and the robustness of the effect is debatable. The use of garlic for hypercholesterolaemia is therefore of questionable value. A more recent update confirmed these conclusions. ${ }^{121}$

\subsubsection{Artichoke}

Hypercholesterolaemia is directly associated with an increased risk for coronary heart disease and other sequelae of atherosclerosis. Artichoke leaf extract (ALE) has been implicated in lowering cholesterol levels. Whether ALE is truly effective for this indication, however, is still a matter of debate. The objective of this Cochrane review ${ }^{122}$ was to assess the evidence of ALE versus placebo or reference medication for treating hypercholesterolaemia defined as mean total cholesterol levels of at least $5.17 \mathrm{mmol} / \mathrm{L}(200 \mathrm{mg} / \mathrm{dL})$. We searched the Cochrane Central Register of Controlled Trials 2008 Issue 2,MEDLINE, EMBASE, AMED and CINAHL from their respective inception until June 2008; CISCOMuntil June 2001. Reference lists of articles were checked.Manufacturers of preparations containing artichoke extract and experts on the subject were contacted. Randomised controlled trials (RCTs) of ALE mono-preparations compared with placebo or reference medication for patients with hypercholesterolaemia were included. Trials assessing ALE as one of several active components in a combination preparation or as a part of a combination treatment were excluded. Data were extracted systematically and methodological quality was evaluated using a standard scoring system and the Cochrane risk of bias assessment. The screening of studies, selection, data extraction and assessment of methodological quality were performed independently by two reviewers. Disagreements in the evaluation of individual trials were resolved through discussion. Three RCTs (262 participants) met all inclusion criteria. In one trial the total cholesterol level in participants receiving ALE decreased by $4.2 \%$ from $7.16(0.62) \mathrm{mmol} / \mathrm{L}$ to $6.86(0.68) \mathrm{mmol} / \mathrm{L}$ after 12 weeks and increased from $6.90(0.49) \mathrm{mmol} / \mathrm{L}$ to $7.04(0.61) \mathrm{mmol} / \mathrm{L}$ in patients receiving placebo, the total difference being statistically significant $(\mathrm{P}=0.025)$. In a further trial ALE reduced total cholesterol levels by $18.5 \%$ from $7.74 \mathrm{mmol} / \mathrm{L}$ to $6.31 \mathrm{mmol} / \mathrm{L}$ after $42 \pm 3$ days of treatment whereas the placebo reduced cholesterol by $8.6 \%$ from $7.69 \mathrm{mmol} / \mathrm{L}$ to $7.03 \mathrm{mmol} / \mathrm{L}(\mathrm{P}=0.00001)$. Another trial did state that ALE significantly reduced blood cholesterol compared with placebo in a sub-group of patients with baseline total cholesterol levels of more than $230 \mathrm{mg} / \mathrm{dL}(\mathrm{P}<0.05)$. Trial reports indicate mild, transient and infrequent adverse events. Some data from clinical trials assessing ALE for treating hypercholesterolaemia exist. There is an indication that ALE has potential in lowering cholesterol levels, the evidence is, however, as yet not convincing. The limited data on safety suggest only mild, transient and infrequent adverse events with the short term use of ALE.

\subsubsection{Any herbal medicine}

In this article we wanted to systematically review the clinical evidence for herbal medicinal products in the treatment of hypercholesterolaemia. ${ }^{123}$ Systematic literature searches were conducted in 6 electronic databases. The reference lists of all papers and our files were searched for more relevant publications. Experts in the field and manufacturers of identified herbal medicinal products were 
contacted for published and unpublished data. No language restrictions were imposed. All randomised clinical trials of serum cholesterol reduction, in which mono-preparations of herbal medicinal products were administered as supplements to human subjects, were included. Twenty-five randomised clinical trials involving 11 herbal medicinal products were identified. Guggul (Commiphora mukul), fenugreek (Trigonella foenum-graecum), red yeast rice, and artichoke (Cynara scolymus) have been most extensively studies and have demonstrated reductions in total serum cholesterol levels between $10 \%$ and $33 \%$. The methodological quality as assessed by the Jadad score was less than 3 (maximum, 5) for 13 of the 25 trials. It was concluded that many herbal medicinal products have potential hypercholesterolaemic activity and encouraging safety profiles. However, only a limited amount of clinical research exists to support their efficacy. Further research is warranted to establish the value of these extracts in the treatment of hypercholesterolaemia.

\subsection{Hypertension (9 systematic reviews)}

\subsubsection{Any therapy}

Many hypertensive patients try complementary/alternative medicine for blood pressure control. Based on extensive electronic literature searches, the evidence from clinical trials was summarised. ${ }^{124}$ Numerous herbal remedies, non-herbal remedies and other approaches have been tested and some seem to have antihypertensive effects. The effect size is usually modest, and independent replications are frequently missing. The most encouraging data to pertain to garlic, autogenic training, biofeedback and yoga. More research is required before firm recommendations can be offered.

\subsubsection{Aromatherapy}

The objective of this review ${ }^{125}$ is to systematically review the evidence for the effectiveness of aromatherapy in the treatment of high blood pressure. Twelve databases were searched from their inception through December 2009. Controlled trials testing aromatherapy in patients with hypertension of any origin that assessed blood pressure were considered. The selection of studies, data extraction and validations were performed independently by two reviewers. One randomized clinical trial (RCT) and four non-randomized controlled clinical trials (CCTs) met our inclusion criteria. The one RCT included tested the effects of aromatherapy as compared with placebo and showed significant reduction of systolic blood pressure and diastolic blood pressure. All of the four CCTs showed favourable effects of aromatherapy. However, all of the CCTs also had a high risk of bias. In conclusion, the existing trial evidence does not show convincingly that aromatherapy is effective for hypertension. Future studies should be of high quality with a particular emphasis on designing an adequate control intervention.

\subsubsection{Autogenic training}

The aim of a further systematic review of autogenic training ${ }^{126}$ was to evaluate all controlled trials testing its effects on blood pressure. A search for controlled trials was carried out in four databases. Five such trials reported anti-hypertensive effects of autogenic training. The majority of trials were methodologically flawed. We concluded therefore that the notion that autogenic training lowers blood pressure is not based on the evidence from rigorous clinical trials. 


\subsubsection{Chiropractic}

Chiropractic spinal manipulation is claimed to be an effective treatment for hypertension. This systematic review ${ }^{127}$ is aimed at evaluating the evidence from randomised clinical trials on this subject. Electronic literature searches were conducted in six databases. No language restrictions were applied. The methodological quality of the trials was evaluated with the Jadad score. Four studies met the pre-defined inclusion criteria. Their methodological quality was mostly poor. The results were mixed. Collectively these data fail to convincingly demonstrate a hypertensive effect of chiropractic spinal manipulation in patients. Until evidence to the contrary emerges, chiropractic spinal manipulation cannot be considered an effective treatment for hypertension.

\subsubsection{Ginseng}

Ginseng is one of the most-widely used herbal remedies. This systematic review ${ }^{128}$ evaluates the current evidence for its use in the reducing blood pressure (BP) in patients with hypertension. Systematic searches of 12 electronic databases were conducted without language restrictions. All randomized clinical trials (RCTs) of ginseng as a treatment for hypertension were candidates for inclusion. Methodological quality was assessed using the Cochrane risk of bias. Five RCTs met the inclusion criteria. The risk of bias was low in most of the trials. Four of the included RCTs compared the effectiveness of ginseng to placebo. The meta-analysis of these data failed to show a statistically significant acute effect on systolic BP (SBP) or diastolic BP (DBP). However, subgroup analyses showed beneficial effects of Korean red ginseng (KRG) on both SBP $(n=54$, mean difference [MD], $6.52 ; 95 \%$ confidence interval [CI], -9.99 to $-3.04 ; \mathrm{p}=0.0002)$ and $\mathrm{DBP}(\mathrm{n}=54, \mathrm{MD},-5.21 ; 95 \% \mathrm{CI}$, 7.90 to- 2.51; $\mathrm{p}=0.0001$ ). Two RCTs tested the long-term effects of ginseng for BP for 24 hours. One of these trials failed to show any benefits of KRG compared to no treatment, and the other failed to show superior effects of North American ginseng compared to placebo. Adverse events with ginseng were none in one trial or not assessed. Collectively, these RCTs provide limited evidence for the acute effectiveness of KRG in the treatment of high BP. The total number of RCTs included in the analysis and the total sample size were insufficient to draw definitive conclusions. More rigorous studies are warranted.

\subsubsection{Moxibustion}

Moxibustion is a traditional East Asian medical therapy that uses the heat generated by burning herbal preparations containing Artemisia vulgaris to stimulate acupuncture points. The aim of this review ${ }^{129}$ was to evaluate previously published clinical evidence for the use of moxibustion as a treatment for hypertension. We searched 15 databases without language restrictions from their respective dates of inception until March 2010. We included randomized controlled trials (RCTs) comparing moxibustion to either antihypertensive drugs or no treatment. The risk of bias was assessed for each RCT. During the course of our search, we identified 519 relevant articles. A total of 4 RCTs met all the inclusion criteria, two of which failed to report favorable effects of moxibustion on blood pressure (BP) compared to the control (antihypertensive drug treatment alone). However, a third RCT showed significant effects of moxibustion as an adjunct treatment to antihypertensive drug therapy for lowering BP compared to antihypertensive drug therapy alone. The fourth RCT included in this review addressed the immediate BP-lowering effects of moxibustion compared to no treatment. None of the included RCTs reported the sequence generation, allocation concealment and evaluator blinding. In conclusion, there is insufficient evidence to suggest that moxibustion is an effective treatment for hypertension. Rigorously designed trials are warranted to answer the many remaining questions. 


\subsubsection{Qigong}

Our objective was to assess systematically the clinical evidence of qigong for hypertension. ${ }^{130}$ Databases were searched up to August 2006. All randomized clinical trials (RCTs) testing qigong in patients with hypertension of any origin and assessing clinically relevant outcomes were considered. Trials using any type of control intervention were included. The selection of studies, data extraction and quality assessment were performed independently by at least two reviewers. Methodological quality was evaluated using the Jadad score. A total of 121 potentially relevant articles were identified and 12 RCTs were included. Seven RCTs tested qigong in combination with antihypertensive drugs compared with antihypertensive drugs alone. The meta-analysis of two trials reporting adequate data suggested beneficial effects in favour of qigong [weighted mean difference, systolic blood pressure (SBP) S12.1mmHg, 95\% confidence interval (CI) S17.1 to S7.0; diastolic blood pressure $\mathrm{S} 8.5 \mathrm{mmHg}, 95 \%$ CI S12.6 to S4.4]. Qigong was compared with waiting list control in two RCTs and was found to reduce SBP significantly (weighted mean difference $\mathrm{S} 18.5 \mathrm{mmHg}, 95 \%$ CI S23.1 to S13.9). In three further RCTs the comparisons made were: qigong combined with conventional therapy versus muscle relaxation combined with conventional therapy; qigong as a sole treatment versus exercise. All reported positive results in at least some of the relevant outcome measures. The methodological quality of the studies was low. There is some encouraging evidence of qigong for lowering SBP, but the conclusiveness of these findings is limited. Rigorously designed trials are warranted to confirm these results.

\subsubsection{Tai Chi}

The objective of this systematic review ${ }^{131}$ was to assess the evidence for tai chi in reducing resting blood pressure (BP) in the elderly. Databases were searched up to February 2009. All randomized clinical trials (RCTs) testing the effects of tai chi on resting BP in the elderly were considered. The selection of studies, data extraction and validation were performed independently by two reviewers. Methodological quality was evaluated using the Jadad score. A total of 329 potentially relevant articles were identified and four RCTs met the inclusion criteria. One study suggested a significant BP reduction compared with no treatment or wellness education programme, while the others showed no effects compared with resistance exercise and usual activity. Two RCTs failed to show a reduction of resting BP compared with aerobic exercise, low impact exercise and no exercise control. In conclusion, the evidence for tai chi in reducing BP in the elderly individuals is limited. Whether tai chi has benefits over exercise is still unclear. The number of trials and the total sample size are too small to draw any firm conclusions. Further rigorous RCTs are warranted.

\subsubsection{Transcendental meditation}

An independent, systematic review was performed of randomised clinical trials of Transcendental Meditation TM for cumulative effects on blood pressure. ${ }^{132}$ Searches were made of electronic databases and the collected papers and official websites of the TM organisation. We included only randomised clinical trials, without confounding co-interventions, which measured the cumulative effects of TM on blood pressure. Six trials met the inclusion criteria but one, reported only in abstract form, could not be evaluated. Procedures for establishing baseline blood pressure were adequate in only one trial. Only one of the trials included a follow-on assessment and only one of the evaluable trials tested the effect of TM in hypertensive individuals. Three of the five evaluable trials reported statistically significant differences between intervention groups. None of the five studies was conducted by independent authors without any affiliation to the TM organisation. We concluded that the randomised clinical trials of TM for the control of blood pressure published to date have important methodological weaknesses and are potentially biased by the affiliation of authors to the 
TM organisation. There is at present in sufficient good-quality evidence to conclude whether or not TM has a cumulative positive effect on blood pressure.

\subsection{Infant colic (1 systematic review)}

Some chiropractors claim that spinal manipulation is an effective treatment for infant colic. This systematic review ${ }^{133}$ was aimed at evaluating the evidence for this claim. Four databases were searched and three randomised clinical trials met all the inclusion criteria. The totality of this evidence fails to demonstrate the effectiveness of this treatment. It is concluded that the above claim is not based on convincing data from rigorous clinical trials.

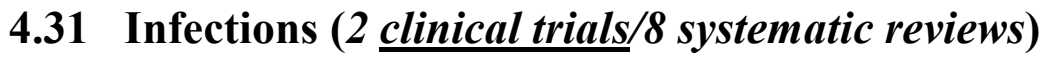

\subsubsection{CAM general}

The objective of this review ${ }^{134}$ was to assess the evidence for the effectiveness of complementary and alternative therapies for preventing or treating influenza or influenza-like illness, including avian influenza. Systematic literature searches were conducted in 5 databases until June 2006; other data sources included bibliographies of located articles, manufacturers of commercially available preparations, and experts in the field. Randomized clinical trials, controlled against placebo or active comparator, were included. Decisions on inclusion, data extraction, and methodological quality assessment were performed independently by 2 reviewers. Fourteen randomized controlled trials testing 7 preparations were included. For Oscillococcinum, $P$. quinquefolium extract, Sambucus nigra, and the herbal combination Kan Jang, 2 or more trials reporting some encouraging data were identified. In conclusion, the effectiveness of any complementary and alternative therapy for treating or preventing seasonal influenza is not established beyond reasonable doubt. Current evidence from randomized controlled trials is sparse and limited by small sample sizes, low methodological quality, or clinically irrelevant effect sizes. For avian influenza, no data are currently available. These results strengthen conventional approaches for seasonal influenza.

\subsubsection{Echinacea}

A systematic review included 12 RCTs of Echinacea products for treating upper respiratory tract infections (URTIs). ${ }^{135}$ With the exception of one study, all suggested Echinacea to be effective. The review also included 6 RCTs of Echinacea for the prevention of URTIs. Again all except one study suggested effectiveness. As the methodological quality of many of these RCTs was low, it was not possible to arrive at a definite conclusion on the value of Echinacea preparation for either indication.

\subsubsection{Herbal mixture}

Herbal expectorants and secretolytic drugs hold a sizeable share of the European market. Therefore it is essential to test their clinical effectiveness and safety. The aim of this study ${ }^{136}$ was to compare the herbal medication Bronchipret ${ }^{\circledR}$ with various other pharmacotherapeutical options for acute bronchitis. The study was designed as a matched-pair comparison of 7783 patients. Clinical outcomes of bronchitis and adverse reactions were documented. The data were evaluated by comparing the treatment success of the test medication and 3 control groups using ordinal regression. The results suggest that clinical effectiveness of Bronchipret ${ }^{\circledR}$ was not less than with synthetic drugs. There was a tendency for better results with Bronchipret ${ }^{\mathbb{B}}$, particularly in the treatment of adults. Similar results were obtained with respect to adverse reactions. Particularly in the adult sub-group, these were markedly less with Bronchipret ${ }^{\circledR}$ compared to synthetic drugs. These findings imply that a 
risk/benefit evaluation would favour Bronchipret ${ }^{\circledR}$ over synthetic drugs for acute bronchitis. Their interpretation is limited through the fact that this study could not be randomised or blinded. The results therefore require confirmation through randomised, double-blind trials.

\subsubsection{Garlic}

In vitro experiments have demonstrated that the growth of Helicobacter pylori can be inhibited by garlic extracts. Epidemiological investigations suggest that regular garlic consumption protects against $H$ pylori infection. Moreover, a large body of evidence implies that a high incidence of garlic usage is associated with a reduced risk of stomach cancer. Using this information, we conducted an uncontrolled pilot study of the use of garlic to treat $H$ pylori infections. ${ }^{137}$ Twenty individuals who previously tested positive for $H$ pylori using the urea breath test received 300-mg tablets of dried garlic powder (LI 114; Lichtwer Pharma, Berlin, Germany) 3 times a day for 8 weeks. Individuals consuming a garlic-rich diet or receiving antibiotic therapy were excluded. All participants were retested for $H$ pylori using the urea breath test after 4 and 8 weeks of treatment. Of the 15 participants who completed the 8-week treatment regimen, the results of the urea breath test were negative for $H$ pylori in only 1 . Subsequently, in several single case studies, we compared crushed tablets with high doses (as much as 1 bulb) of fresh garlic. Ingestion of garlic at doses equivalent to 1 bulb led to acute nausea and vomiting. Our results did not therefore support the hypothesis that garlic is effective in treating $H$ pylori infections.

\subsubsection{Andrographis paniculata}

Acute respiratory infections represent a significant cause of over-prescription of antibiotics and are one of the major reasons for absence from work. The leaves of Andrographis paniculata (Burm. F.) Wall ex Nees (Acanthaceae) are used as a medicinal herb in the treatment of infectious diseases. Systematic literature searches were conducted in six computerised databases and the reference lists of all papers located were checked for further relevant publications. ${ }^{138}$ Information was also requested from manufacturers, the spontaneous reporting schemes of the World Health Organisation and national drug safety bodies. No language restrictions were imposed. Seven double-blind, controlled trials $(n=896)$ met the inclusion criteria for evaluation of efficacy. All trials scored at least three, out of a maximum of five, for methodological quality on the Jadad scale. Collectively, the data suggest that $A$. paniculata is superior to placebo in alleviating the subjective symptoms of uncomplicated upper respiratory tract infection. There is also preliminary evidence of a preventative effect. Adverse events reported following administration of $A$. paniculata were generally mild and infrequent. There were few spontaneous reports of adverse events. A. paniculata may be a safe and efficacious treatment for the relief of symptoms of uncomplicated upper respiratory tract infection; more research is warranted.

\subsubsection{Pelargonium sidoides}

The object of this systematic review ${ }^{139}$ was to critically assess the efficacy of Pelargonium sidoides for treating acute bronchitis. Systematic literature searches were performed in 5 electronic databases: (Medline (1950 - July 2007), Amed (1985 - July 2007), Embase (1974 - July 2007), CINAHL (1982 - July 2007), and The Cochrane Library (Issue 3, 2007) without language restrictions. Reference lists of retrieved articles were searched, and manufacturers contacted for published and unpublished materials. Study selection was done according to predefined criteria. All randomized clinical trials (RCTs) testing P. sidoides extracts (mono preparations) against placebo or standard treatment in patients with acute bronchitis and assessing clinically relevant outcomes were included. Two reviewers independently selected studies, extracted and validated relevant data. Methodological quality was evaluated using the Jadad score. Meta-analysis was performed using a fixed-effect model 
for continuous data, reported as weighted mean difference with $95 \%$ confidence intervals. Six RCTs met the inclusion criteria, of which 4 were suitable for statistical pooling. Methodological quality of most trials was good. One study compared an extract of P. sidoides, EPss7630, against conventional non-antibiotic treatment (acetylcysteine); the other five studies tested EPss7630 against placebo. All RCTs reported findings suggesting the effectiveness of P. sidoides in treating acute bronchitis. Metaanalysis of the four placebo-controlled RCTs suggested that EPss7630 significantly reduced bronchitis symptom scores in patients with acute bronchitis by day 7. No serious adverse events were reported. We concluded that there is encouraging evidence from currently available data that $\mathrm{P}$. sidoides is effective compared to placebo for patients with acute bronchitis.

\subsubsection{Herbal medicines in general}

Many antiviral compounds presently in clinical use have a narrow spectrum of activity, limited therapeutic usefulness and variable toxicity. There is also an emerging problem of resistant viral strains. This systematic review was undertaken to examine the published literature in herbs and plants with antiviral activity, their laboratory evaluation in vitro and in vivo, and evidence of human clinical efficacy. ${ }^{140}$ Independent literature searches were performed on MEDLINE, EMBASE, CISCOM, AMED and Cochrane Library for information on plants and herbs with antiviral activity. There was no restriction on the language of publication. Data from clinical trials of single herb preparations used to treat uncomplicated viral infections were extracted in a standardised, pre-defined manner. Many hundreds of herbal preparations with antiviral activity were identified and the results of one search presented as an example. Yet extracts from only 11 species met the inclusion criteria of this review and have been tested in clinical trials. They have been used in a total of 33 randomised and a further eight non-randomised, clinical trials. Fourteen of these trials described the use of Phyllanthus spp. For the treatment of hepatitis B, seven reported positive and seven reported negative results. The other 10 herbal medicines had each been tested in between one and nine clinical trials. Only four of these 26 trials reported no benefit from the herbal product. We concluded that there remains a need for larger, stringently designed, randomised clinical trials to provide conclusive evidence of their efficacy.

Many hundreds of plant extracts have been tested for in vitro antibacterial activity. This review is a critical evaluation of controlled clinical trials of herbal medicines with antibacterial activity. ${ }^{141}$ Four electronic databases were searched for controlled clinical trials of antibacterial herbal medicines. Data were extracted and validated in a standardised fashion, according to pre-defined criteria, by two independent reviewers. Seven clinical trials met our inclusion criteria. Four of these studies were randomised. Three trials of garlic and cinnamon treatments for Helicobacter pylori infections reported no significant effect. Bacterial infections of skin were treated in four trials. Positive results were reported for an ointment containing tea leaf extract in impetigo contagiosa infections. Two trials of tea tree oil preparations used for acne and methicillin-resistant Staphylococcus aureus, and one trial of Ocimum gratissimum oil for acne, reported results equivalent to conventional treatments. We concluded that few controlled clinical trials have been published and most are methodologically weak. The clinical efficacy of none of the herbal medicines has so far been demonstrated beyond doubt. This area seems to merit further study through rigorous clinical trials.

Traditional medicine has made use of may different plant extracts for treatment of fungal infections and some of these have been tested for in vitro antifungal activity. This systematic review ${ }^{142}$ evaluates antifungal herbal preparations that have been tested in controlled clinical trials. Four electronic databases were searched for controlled clinical trials of antifungal herbal medicines. Data were extracted in a standardized manner by two independent reviewers and are reviewed narratively. Seven clinical trials met our inclusion criteria. Tea tree oil preparations were tested in four randomised clinical trials and some positive outcomes were attributed to the intervention in all trials. 
Solanum species (two trials) and oil of bitter orange preparations (one trial) were compared with conventional treatments. In all cases encouraging results were reported. There are few controlled clinical trials of herbal antifungal medicines. The most thoroughly clinically tested is tea tree oil, which holds some promise. All herbal remedies require further investigation in rigorous clinical trials.

\subsubsection{Other complementary therapies}

Complementary treatments are often used by HIV-infected individuals. Yet little is known about their effectiveness. The aim of this systematic review ${ }^{143}$ was therefore to summarise the published evidence for or against the effectiveness of complementary therapies in HIV-positive people. A comprehensive literature search was conducted to locate all randomised clinical trials of complementary therapies. Data were extracted in a standardised fashion and evaluated critically. Fourteen studies met our pre-defined inclusion/exclusion criteria; 2 of herbal treatments, 5 of vitamins and other supplements, 5 of stress management, one of massage therapy, and one of acupuncture. They fall into 2 broad categories of 'cure' and 'care'. While the former category yields few encouraging results, the latter group of studies is more promising. In particular, stress management may prove to be an effective way to increase the quality of life. It is concluded that few rigorous trials of complementary treatments for HIV exist. Complementary medicine may be applicable in the care for HIV-infected individuals with a view of increasing their quality of life. This notion requires further rigorous investigation.

\subsection{Insomnia (2 systematic reviews)}

\subsection{1 $\underline{\text { Acupuncture }}$}

Auricular acupuncture (AA) is a therapeutic method by which specific points on the auricle are stimulated to treat various conditions. AA is often recommended as treatment for insomnia. The aim of this systematic review ${ }^{144}$ was to evaluate data from randomised, placebo-controlled clinical trials testing the effectiveness of AA for treating insomnia. We searched the literature using 18 databases from their inception to April 2008 without language restrictions. All prospective randomised clinical trials (RCTs) of AA for subjects with insomnia were considered. Methodological quality was assessed using the Jadad score. We identified 433 possible relevant articles, in which include 10 acceptable RCTs. The methodological quality of the trials was generally poor. Magnetic pellets AA was compared with placebo AA in three of the studies. The results suggested beneficial effects on sleep efficiency compared with placebo AA. One RCT tested needle AA compared with placebo AA and failed to show the effectiveness of AA. Four RCTs compared Semen Vaccariae or magnetic pellet AA with conventional drugs (estazolam or diazepam). Favourable effects for AA were found. Two RCTs tested thumbtack needle AA vs. no treatment suggested beneficial effects of AA on a sleep score. We conclude that, because of the paucity and of the poor quality of the data, the evidence for the effectiveness of AA for the symptomatic treatment of insomnia is limited. Further, rigorously designed trials are warranted to confirm these results.

\subsubsection{Valerian}

Our objective was to systematically review the evidence for the effects of the herb valerian (Valeriana officinalis) on insomnia, based on randomised, placebo-controlled, double-blind trials ${ }^{145}$. Literature searches were performed to locate randomised, placebo-controlled, double-blind trials measuring the effect of valerian monopreparations on sleep in human participants. Data were extracted in a standardised manner and methodological quality was assessed by the Jadad score. Nine 
trials were located meeting the selection criteria. The findings of the studies were contradictory and there was great inconsistency between trials in terms of patients, experimental design and procedures and methodological quality. The evidence for valerian as a treatment for insomnia is therefore inconclusive. There is a need for rigorous trials to determine its efficacy.

\subsection{Irritable bowel syndrome (2 systematic reviews)}

\subsubsection{Peppermint}

The objective of this systematic review ${ }^{146}$ was to review the clinical trials of extracts of peppermint (Mentha X piperita L.) as a symptomatic treatment for irritable bowel syndrome (IBS). Peppermint oil is the major constituent of several over-the-counter remedies for symptoms of IBS. As the aetiology of IBS is not known and treatment is symptomatic, there is a ready market for such products. However, evidence to support their use is sparse. Five computerised literature searches were performed to identify all randomised clinical trials of peppermint oil for IBS. There were no restrictions on the language of publication. Data were extracted in a standardised, pre-defined fashion, independently by both authors. Eight randomised, controlled trials were located. Collectively they indicate that peppermint oil could be efficacious for symptom relief in IBS. A meta-analysis of five placebo-controlled, double blind trials seems to support this notion. In view of the methodological flaws associated with most studies, no definitive judgement about efficacy can be given. Thus the role of peppermint oil in the symptomatic treatment of IBS has so far not been established beyond reasonable doubt. Well-designed and carefully executed studies are needed to clarify the issue.

\subsubsection{Herbal medicines}

A systematic review was published with a wider focus on all herbal treatments for this condition. ${ }^{147}$ Encouraging data were found for peppermint (see above), asafoetida, nux vomica, ispaghula and two combination products, Iberogast ${ }^{\circledR}$ and Appital ${ }^{\circledR}$. For the latter remedies, there are too few trials for the evidence to be conclusive.

\subsection{Labour pain (2 systematic reviews)}

Acupuncture is widely used to alleviate symptoms in a variety of painful conditions. In obstetrics and gynaecology, acupuncture has also been applied to a range of conditions including labour pain. This systematic review ${ }^{148}$ aims to critically evaluate the evidence on the analgesic effect of acupuncture during labour. Computerised literature searches of 7 databases were performed for randomised clinical trials (RCTs) of acupuncture involving needle insertion for pain during labour. Three RCTs were identified and their methodological quality was generally good. Two RCTs compared adjunctive acupuncture with usual care only and reported a reduction of meperidine and/or epidural analgesia. One placebo acupuncture controlled trial showed a statistically significant difference in both subjective and objective outcome measures of pain. No adverse events were reported in any of the trials. It is concluded that the evidence for acupuncture as an adjunct to conventional pain control during labour is promising but, because of paucity of trial data, not convincing. Further research is warranted to clearly define its place in labour pain management.

We updated the evidence for or against acupuncture for labour pain management. ${ }^{149}$ Nineteen electronic databases, including English, Korean, Japanese, and Chinese databases, were systematically searched. All randomised controlled trials (RCTs) involving women receiving acupuncture alone, or as an adjunct to conventional analgesia, for pain relief in labour were 
considered. Pain intensity on a 100-mm visual analogue scale (VAS; 0, no pain; 100, worst pain) and use of other analgesic methods were used as primary outcomes, and for statistical pooling. Maternal/fetal outcomes were secondary outcomes, and adverse events were also recorded. Risk of bias was assessed regarding randomisation, allocation concealment, blinding, incomplete outcome data, selective outcome reporting, and other biases. Ten RCTs involving 2038 women were included. VAS for pain intensity data were available in seven studies; the meta-analysis shows that acupuncture was not superior to minimal acupuncture at 1 hour (pooled mean difference $-8.02 ; 95 \%$ CI $-21.88,5.84 ; \mathrm{I}(2)=94 \%)$ and at 2 hours $(-10.15 ; 95 \% \mathrm{CI}-23.18,2.87$; $(2)=92 \%)$. Patients reported significantly reduced pain by 4 and $6 \%$ during electroacupuncture (EA) treatment at 15 (4.09; 95\% CI -8.05, -0.12) and 30 minutes (-5.94; 95\% CI -9.83, -2.06), compared with placebo EA, but the effect was not maintained afterwards. Compared with no intervention, acupuncture reduced pain by only $11 \%$ for the first 30 minutes $(-10.56$; $95 \%$ CI $-16.08,-5.03)$. In trials where acupuncture was compared with conventional analgesia, women receiving acupuncture required less meperidine (pooled risk ratio $0.20 ; 95 \%$ CI $0.12,0.33)$ and other analgesic methods $(0.75 ; 95 \%$ CI $0.66,0.85)$. No acupuncture-related adverse events were reported. Most trials did not blind participants, care providers and/or evaluators. In conclusion, the evidence from RCTs does not support the use of acupuncture for controlling labour pain. The primary studies are diverse and often flawed. Further research seems warranted.

\subsection{Low back pain (1 survey/6 systematic reviews/1 editorial)}

\subsubsection{Expert opinion}

Expert opinions on the use of complementary therapies for low back pain could be helpful until more data from randomised clinical trials become available. A postal questionnaire survey was designed to generate opinion from a systematically identified expert panel on the clinical effectiveness of complementary therapies for low back pain. ${ }^{150}$ Computerised searches were conducted to systematically identify by objective criteria 50 clinical experts on low back pain. Each panel member received a questionnaire to assess the perceived clinical effectiveness of complementary therapies for 4 defined categories of low back pain. For acute uncomplicated low back pain, osteopathy and chiropractic were rated as effective by most experts. For chronic uncomplicated low back pain, most experts perceived acupuncture as effective. Experts perceived homoeopathy generally as ineffective for any type of low back pain. Clinical experience with herbalism as a treatment for low back pain was insufficient to form an opinion.

\subsubsection{CAM general}

Back pain is the most common reason for using complementary therapies. This analysis of the trial evidence $^{151}$ is aimed at determining whether the evidence base for or against complementary therapies for back pain is getting stronger. Two series of systematic reviews conducted with the same methodology 5 years apart were compared. The results suggest that the weight of the evidence has increased between 2000 and 2005 for a number of interventions. The direction of the evidence, however, remained unchanged for all but one therapy. We conclude that the value of complementary therapies in the management of back pain remains encouraging but not fully convincing.

\subsubsection{Acupuncture}

Acupuncture is commonly used to treat back pain. The objective of our systematic review ${ }^{152}$ was to perform a meta-analysis of trials of acupuncture for the treatment of back pain. A systematic 
literature search was conducted to retrieve all randomised clinical trials of any form of acupuncture for any type of back pain. The adequacy of the acupuncture treatment was blindly assessed by 6 experienced acupuncturists. The main outcome measure for the meta-analysis was the proportion of patients whose symptoms were improved at the end of treatment. Twelve studies were included, of which 9 presented data suitable for meta-analysis. There was little agreement between acupuncturists as to the adequacy of the acupuncture techniques employed in these trials. The odds ratio of improvement with acupuncture compared with control intervention was 2.30 (95\% confidence interval, 1.28 to 4.13). For sham-controlled, evaluator-blinded studies, the odds ratio was 1.37 (95\% confidence interval, 0.84 to 2.25 ). Acupuncture was shown to be superior to various control interventions, although there is insufficient evidence to state whether it is superior to placebo.

An update assessed acupuncture's effectiveness for treating low back pain. ${ }^{153}$ Randomized, controlled trials were identified through searches of MEDLINE, Cochrane Central, EMBASE, AMED, CINAHL, CISCOM, and GERA databases through August 2004. Additional data sources included previous reviews and personal contacts with colleagues. Randomized, controlled trials were included comparing needle acupuncture with sham acupuncture, other sham treatments, no additional treatment, or another active treatment for patients with low back pain. Data were dually extracted for the outcomes of pain, functional status, overall improvement, return to work, and analgesic consumption. In addition, study quality was assessed. The 33 randomized, controlled trials that met inclusion criteria were subgrouped according to acute or chronic pain, style of acupuncture, and type of control group used. For the primary outcome of short-term relief of chronic pain, the metaanalyses showed that acupuncture is significantly more effective than sham treatment (standardized mean difference, 0.54 [95\% CI, 0.35 to 0.73$]$; 7 trials) and no additional treatment (standardized mean difference, 0.69 [CI, 0.40 to 0.98 ]; 8 trials). For patients with acute low back pain, data are sparse and inconclusive. Data are also insufficient for drawing conclusions about acupuncture's short-term effectiveness compared with most other therapies. We concluded that acupuncture effectively relieves chronic low back pain. No evidence suggests that acupuncture is more effective than other active therapies.

\subsubsection{Chiropractic}

The aim of this systematic review ${ }^{154}$ was to critically evaluate the evidence for or against the effectiveness of chiropractic spinal manipulation for back pain. Five independent literature searches were carried out and bibliographies were searched. Only randomized clinical trials of chiropractic spinal manipulation with patients suffering from back pain were included. We extracted data on trial design, methodological quality, sample size, patient characteristics, nature of intervention, outcome measures, follow-up and results. Twelve studies could be included. They related to all forms of back pain. Many trials had significant methodological shortcomings. Some degree of superiority of chiropractic spinal manipulation over control interventions was noted in 5 studies. More recent trials and those with adequate follow-up periods tended to be negative. We concluded that the effectiveness of chiropractic spinal manipulation is not supported by compelling evidence from the majority of randomized clinical trials.

\subsubsection{Massage therapy}

Massage therapy is also frequently employed for low back pain. The aim of this systematic review ${ }^{155}$ was to evaluate the evidence for or against its efficacy in this indication. Four randomised clinical trials were located in which massage was tested as a sole therapy for low back pain. All were burdened with major methodological flaws. One of these studies suggests that massage is superior to no treatment. Two trials imply that it is equally effective as spinal manipulation or transcutaneous electrical stimulation (TENS). One study suggests that it is less effective than spinal manipulation. It 
was therefore concluded that too few trials of massage therapy exist for a reliable evaluation of its efficacy, but it seems to have some potential as a therapy for low back pain.

As several more trials have emerged since the publication of the above article, it became necessary to update it. ${ }^{156}$ With two additional positive trials, the conclusion was as follows: "massage therapy appears to be an effective treatment for low back pain". More recently this view was reiterated in a BMJ Editorial which cautiously concluded that "the evidence for massage as a symptomatic relief of back pain is encouraging but not compelling". ${ }^{157}$

\subsubsection{Spa therapy}

Low back pain is a major public health concern and complementary treatments are frequently used for this condition. The objective of this systematic review ${ }^{158}$ and meta-analysis was to assess the evidence for or against the effectiveness of spa therapy and balneotherapy for treating low back pain. Systematic searches were conducted on Medline, Embase, Amed Cochrane Central, the UK National Research Register and ClincalTrials.gov (all until July 2005). Hand searches were performed and experts contacted. Methodological quality was assessed using a standard scale. Five randomized clinical trials met all inclusion criteria. Quantitative data synthesis was performed. The data for spa therapy, assessed on a $100 \mathrm{~mm}$ visual analogue scale (VAS), suggest significant beneficial effects compared with waiting list control groups (weighted mean difference $26.6 \mathrm{~mm}, 95 \%$ confidence interval 20.4-32.8, $\mathrm{n}=442$ ) for patients with chronic low back pain. For balneotherapy the data, assessed on a $100 \mathrm{~mm}$ VAS, also suggest beneficial effects compared with control groups (weighted mean difference $18.8 \mathrm{~mm}, 95 \%$ confidence interval 10.3-27.3, $\mathrm{n}=138$ ). Even though the data are scarce, there is encouraging evidence suggesting that spa therapy and balneotherapy may be effective for treating patients with low back pain. These data are not compelling but warrant rigorous largescale trials.

\subsection{Menopausal symptoms (7 systematic reviews/1 clinical trial)}

\subsubsection{Any therapy}

The use of complementary and alternative medicine (CAM) among menopausal women has increased in the last years. This review ${ }^{159}$ examines the evidence from systematic reviews, RCTs and epidemiological studies of CAM in the treatment of menopausal symptoms. Some evidence exists in favour of phytosterols and phytostanols for diminishing LDL and total cholesterol in postmenopausal women. Similarly, regular fiber intake is effective in reducing serum total cholesterol in hypercholesterolemic postmenopausal women. Clinical evidence also exists on the effectiveness of vitamin $\mathrm{K}$, a combination of calcium and vitamin $\mathrm{D}$ or a combination of walking with other weightbearing exercise in reducing bone mineral density loss and the incidence of fractures in postmenopausal women. Black cohosh appears to be effective therapy for relieving menopausal symptoms, primarily hot flashes, in early menopause. Phytoestrogen extracts, including isoflavones and lignans, appear to have only minimal effect on hot flashes but have other positive health effects, e.g. on plasma lipid levels and bone loss. For other commonly used CAMs, e.g. probiotics, prebiotics, acupuncture, homeopathy and DHEA-S, randomized, placebo-controlled trials are scarce and the evidence is unconvincing. More and better RCTs testing the effectiveness of these treatments are needed. 


\subsubsection{Acupuncture}

The objective of this systematic review ${ }^{160}$ was to assess the effectiveness of acupuncture as a treatment option for menopausal hot flushes. We have searched the literature using 17 databases from inception to October 10, 2008, without language restrictions. We included randomized clinical trials (RCTs) of acupuncture versus sham acupuncture. Their methodological quality was assessed using the modified Jadad score. In total, six RCTs could be included. Four RCTs compared the effects of acupuncture with penetrating sham acupuncture on non-acupuncture points. All of these trials failed to show specific effects on menopausal hot flush frequency, severity or index. One RCT found no effects of acupuncture on hot flush frequency and severity compared with penetrating sham acupuncture on acupuncture points that are not relevant for the treatment of hot flushes. The remaining RCT tested acupuncture against non-penetrating acupuncture on non-acupuncture points. Its results suggested favourable effects of acupuncture on menopausal hot flush severity. However, this study was too small to generate reliable findings. In conclusion, sham-controlled RCTs fail to show specific effects of acupuncture for control of menopausal hot flushes. More rigorous research seems warranted.

\subsubsection{Herbal treatments often recommended for menopausal symptoms}

Many herbal treatments are being recommended for menopausal symptoms. ${ }^{161}$

$\begin{array}{lll}\text { Alfalfa } & \text { Dandelion } & \text { Catstraw } \\ \text { Ayurvedic medicine } & \text { Dong quai } & \text { Parsley } \\ \text { Black cohosh } & \text { False unicorn } & \text { Passionflower } \\ \text { Blackhaw bark } & \text { Fennel } & \text { Peppermint } \\ \text { Blessed thistle } & \text { Flaxseed oil } & \text { Raspberry } \\ \text { Calendula } & \text { Ginger } & \text { Soy } \\ \text { Cayenne pepper } & \text { Ginkgo biloba } & \text { Valerian } \\ \text { Celery } & \text { Ginseng } & \text { Vitex agnus castus } \\ \text { Chamomile } & \text { Gotu kola } & \text { Wild yam } \\ \text { Chinese herbal mixtures } & \text { Liquorice } & \\ \text { Cimicifuga racemosa } & \text { Motherwort } & \end{array}$

The efficacy and safety of those herbal medicinal products for which trial data exists was reviewed. ${ }^{161}$ Some evidence was found for the HMPs listed below. However, this evidence was not fully convincing for any of these treatments.

- black cohosh

- dong quai

- evening primrose

- ginseng

- kava

- phyoestrogens

- several herbal mixtures. 
Many postmenopausal women use herbal remedies and dietary supplements to counteract menopausal symptoms, including the decline in cognitive function. The aim of this systematic review $^{162}$ is to evaluate the evidence regarding the efficacy of herbal and dietary supplements on cognition in menopause. Randomized clinical trials (RCTs) of herbal medicines and dietary supplements were identified using the Medline, EMBASE, AMED, PsycINFO, CINAHL and The Cochrane Library 2010 (Issue 2) electronic databases and by hand searches. Data were independently extracted and evaluated by two reviewers. Risk of bias was assessed by two independent reviewers using the Cochrane Collaboration tool. welve RCTs were included and five of these suggest that isoflavone, soy and Gingko biloba supplementation may improve cognition in postmenopausal women. However, most of the included studies had serious methodological flaws which demand a cautious interpretation of these findings. In conclusion, the evidence that herbal and dietary supplements might positively affect the cognitive decline during the menopause is not compelling

\subsubsection{Cimicifuga racemosa}

Cimicifuga racemosa has long been advocated as an alternative to hormone-replacement therapy. ${ }^{163}$ However, recent experimental studies and a clinical trial have raised some questions about its use. The aim of this systematic review was principally to summarise the trial evidence regarding the efficacy of $C$. racemosa in the treatment of menopausal symptoms. In addition, we also explored the evidence relating to the mechanism of action of this herbal medicine. Searches of seven computerised databases were performed to identify all randomised clinical trials (RCTs) on $C$. racemosa as well as animal and in vitro experiments. No language restrictions were imposed. Data were extracted by both authors according to pre-defined criteria and are summarised in narrative form. Four RCTs of C. racemosa as a treatment of menopausal symptoms were included. They yielded no compelling evidence for the efficacy of $C$. racemosa on menopausal symptoms. The small beneficial effects observed in some studies could be explained by a central activity, and an oestrogenic activity cannot be completely excluded. It was concluded that, despite plausible mechanisms of action of $C$. racemosa, its clinical efficacy for the treatment of menopausal symptoms has not been convincingly demonstrated through rigorous clinical trials. Additional rigorous RCTs and biochemical and chemical investigations are warranted.

The purpose of this update ${ }^{164}$ of our previous systematic review is to evaluate the clinical evidence for or against the efficacy of black cohosh in alleviating menopausal symptoms. Five computerized databases (Medline, Embase, Amed, Phytobase and Cochrane Library) were searched to identify all clinical data that provided evidence on the efficacy of C. racemosa. Bibliographies of the articles thus located were scanned for further relevant publications. Only double blind, randomized, clinical trials (RCTs) were included in the evaluation of efficacy. No language restrictions were imposed.

Trials were excluded if they did not focus on menopausal problems, they included women suffering medically induced menopause, they did not use black cohosh monopreparations, or they did not use placebo or a standard drug treatment for the control group. Six studies with a total of 1112 peri- and post-menopausal women met our inclusion criteria. The evidence from these RCTs does not consistently demonstrate an effect of black cohosh on menopausal symptoms; a beneficial effect of black cohosh on peri-menopausal women cannot be excluded. The efficacy of black cohosh as a treatment for menopausal symptoms is uncertain and further rigorous trials seem warranted.

\subsubsection{Red clover}

This systematic review ${ }^{165}$ critically assesses the evidence of supplements containing Trifolium pratense (red clover) isoflavones in the reduction of hot flush frequency in menopausal women. 
Systematic literature searches were performed in (Medline (1951 - April 2006), Embase (1974 April 2006), CINAHL (1982 - April 2006), Amed (1985 - April 2006) and The Cochrane Library (Issue 2, 2006). Reference lists located were checked for further relevant publications. Experts in the field and manufacturers of identified products were contacted for unpublished material. No language restrictions were imposed. Studies were selected according to predefined inclusion and exclusion criteria. All randomized clinical trials of monopreparations containing T. pratense isoflavones for treating hot flushes were included. Study selection, data extraction and validation were performed by at least two reviewers with disagreements being settled by discussion. Weighted means and $95 \%$ confidence intervals were calculated and sensitivity analyses were performed.

Seventeen potentially relevant articles were retrieved for further evaluation. Five were suitable for inclusion in the meta-analysis. The meta-analysis indicates a reduction in hot flush frequency in the active treatment group (40-82 $\mathrm{mg}$ daily) compared with the placebo group (weighted mean difference -1.5 hot flushes daily; $95 \% \mathrm{CI}-2.94$ to $0.03 ; \mathrm{p}=0.05$ ). There is evidence of a marginally significant effect of $T$. pratense isoflavones for treating hot flushes in menopausal women. Whether the size of this effect can be considered clinically relevant is unclear. Whereas there is no apparent evidence of adverse events during short-term use, there are no available data on the safety of longterm administration.

\subsubsection{Reflexology}

Clinical experience suggests that reflexology may have beneficial effects on the symptoms occurring in menopausal women, particularly psychological symptoms. This study aims to examine that effect rigorously. ${ }^{166}$ A randomised controlled trial with two parallel arms was conducted in the School of Complementary Health in Exeter, Devon, UK. Seventy-six women, aged between 45 and 60 years, reporting menopausal symptoms were included. Women were randomised to receive nine sessions of either reflexology or non-specific foot massage (control) by four qualified reflexologists given over a period of 19 weeks. Main outcome measures were The Women's Health Questionnaire (WHQ), the primary measures being the subscores for anxiety and depression. Severity (visual analogue scale, VAS) and frequency of flushes and night sweats. Mean (SD) scores for anxiety fell from $0.43(0.29)$ to $0.22(0.25)$ in the reflexology group and from $0.37(0.27)$ to $0.27(0.29)$ in the control group over the course of the treatment. Mean (SD) scores for depression fell from 0.37 $(0.25)$ to $0.20(0.24)$ in the reflexology group and from $0.36(0.23)$ to $0.20(0.210$ in the control (foot massage) group over the same period. For both scores there was strong evidence of a time effect (P $<0.001)$ but no evidence of a time-group interaction $(\mathrm{P}>0.2)$. Similar changes were found for severity of hot flushes and night sweats. In the control group, 14/37 believed they had not received true reflexology. It was concluded that foot reflexology was not shown to be more effective than non-specific foot massage in the treatment of psychological symptoms occurring during the menopause.

\subsubsection{Yoga}

The aim of this study was to assess the effectiveness of yoga as a treatment option for menopausal symptoms. We searched the literature using 14 databases from their inception to July 2008 and included all types of clinical studies regardless of their design. The methodological quality of all studies was assessed using a modified Jadad score. Seven studies met our inclusion criteria. Two randomized clinical trials compared the effects of yoga with those of walking or physical exercise. The meta-analysis of these data failed to show specific effects of yoga on menopausal complaints including psychological, somatic, and vasomotor symptoms. Two randomized clinical trials found no effects of yoga on total menopausal symptoms compared with wait-list control or no treatment. The remaining studies were either non-randomized $(n=1)$ or uncontrolled clinical trials $(n=3)$. They reported favorable effects of yoga on menopausal symptoms. These data collectively show that 
the results of rigorous studies of the effects of yoga for menopausal symptoms are unconvincing. In conclusion, the evidence is insufficient to suggest that yoga is an effective intervention for menopause. Further research is required to investigate whether there are specific benefits of yoga for treating menopausal symptoms.

\subsection{Multiple sclerosis (1 systematic review)}

Multiple sclerosis (MS) is a chronic disease of the central nervous system without a known cure. Thus the strength of using complementary and alternative therapies (CATs) for the treatment of MS symptoms lies in its palliative care and this is borne out by the popularity of these treatments amongst MS sufferers. This review ${ }^{167}$ is aimed at determining whether this use is supported by evidence of effectiveness from rigorous clinical trials. Database literature searches were performed and papers were extracted in a pre-defined manner. 12 randomised clinical trials were located that investigated a CAT for MS: nutritional therapy (4), massage (1), Feldenkrais bodywork (1), reflexology (1), magnetic field therapy (2), neural therapy (1) and psychological counselling (2). The evidence is not compelling for any of these therapies with many trials suffering from significant methodological flaws. There is evidence to suggest some benefit of nutritional therapy for the physical symptoms of MS. Magnetic field therapy and neural therapy appear to have a short-term beneficial effect on the physical symptoms of MS. Massage/bodywork and psychological counselling seem to improve depression, anxiety and self-esteem. The effectiveness for other CATs is unproven at this time. Further investigations are needed in the form of rigorous large-scale trials.

\subsection{Myofascial pain (1 systematic review)}

The objective of this systematic review was to establish whether there is evidence for or against the efficacy of needling as a treatment approach for myofascial trigger point pain. ${ }^{168}$ All randomised, controlled trials in which some form of needling therapy was used to treat myofascial pain were included. Two reviewers independently extracted data concerning trial methods, quality and outcomes. Twenty-three papers were located. No trials were of sufficient quality or design to test the efficacy of any needling technique beyond placebo in the treatment of myofascial pain. Eight of the 10 trials comparing injection of different substances and all 7 higher quality trials found that the effect was independent of the injected substance. All 3 trials that compared dry needling with injection found no difference in effect. The conclusions were as follows. Direct needling of myofascial trigger points appears to be an effective treatment, but the hypothesis that needling therapies have efficacy beyond placebo is neither supported nor refuted by the evidence from clinical trials. Any effect of these therapies is likely because of the needle or placebo rather than the injection of either saline or active drug. Controlled trials are needed to investigate whether needling has an effect beyond placebo on myofascial trigger point pain.

\subsection{Nausea and vomiting (3 systematic reviews/1 clinical trial)}

\subsubsection{Ginger}

Conventional antiemetics are burdened with the potential of teratogenic effects during the critical embryogenic period of pregnancy. Thus, a safe and effective medication would be a welcome addition to the therapeutic repertoire. This systematic review ${ }^{169}$ was aimed at assessing the evidence for or against the efficacy and safety of ginger (Zingiber officinale) therapy for nausea and vomiting during pregnancy. Systematic literature searches were conducted in 3 computerized databases 
(MEDLINE, EMBASE, and Cochrane Library), and the reference lists of all papers located were checked for further relevant publications. For the evaluation of efficacy, only double-blind, randomized controlled trials (RCTs) were included. All retrieved clinical data, includinguncontrolled trials, case reports, observational studies, and RCTs, were included in the review of safety. Six double-blind RCTs with a total of 675 participants and a prospective observational cohort study $(\mathrm{n}=187)$ met all inclusion criteria. The methodological quality of 4 of 5 RCTs was high. Four of the 6 RCTs $(n=246)$ showed superiority of ginger over placebo; the other 2 RCTs $(n=429)$ indicated that ginger was as effective as the reference drug (vitamin B6) in relieving the severity of nausea and vomiting episodes. The observational study retrieved and RCTs (including follow-up periods) showed the absence of significant side effects or adverse effects on pregnancy outcomes. There were no spontaneous or case reports of adverse events during ginger treatment in pregnancy. We concluded that ginger may be an effective treatment for nausea and vomiting in pregnancy. However, more observational studies, with a larger sample size, are needed to confirm the encouraging preliminary data on ginger safety

\subsubsection{Herbal medicine}

Ginger (Zingiber officinale) is often advocated as being beneficial for nausea and vomiting. Whether the herb is truly efficacious for this condition is, however, still a matter for debate. We have performed a systematic review of the evidence from randomised clinical trials for or against the efficacy of ginger for nausea and vomiting. ${ }^{170}$ Six studies met all inclusion criteria and were reviewed. Three on postoperative nausea and vomiting were identified and two of these suggested that ginger was superior to placebo and equally as effective as metoclopramide. The pooled absolute risk reduction for the incidence of postoperative nausea, however, indicated a non-significant difference between the ginger and placebo groups for $1 \mathrm{~g}$ ginger taken before operation (absolute risk reduction 0.052 (95\% confidence interval -0.082 to 0.186$)$ ). One study was found for each of the following conditions: seasickness, morning sickness and chemotherapy-induced nausea. These studies collectively favoured ginger over placebo.

\subsubsection{Acupressure/acupuncture}

In contrast to treatment with acupuncture there are no conceivable direct risks associated with acupressure therapy; tissue trauma or infection in particular are excluded. Unlike studies of acupuncture, trials in acupressure do not run into insurmountable difficulties when a double-blind and placebo controlled design is called for. If, for instance, the relevant acupoint is on the wrist, bands with incorporated studs worn by the patients can be used to apply the pressure. In this case, the sham group can be instructed to wear the bands in a way that the wrong point is stimulated; alternatively, bands without studs can be used as a credible placebo which lends itself to doubleblinding. A best evidence analysis ${ }^{171}$ suggested that acupressure is an effective treatment for nausea and vomiting due to various conditions.

The objective of this study was to compare acupuncture with sham (placebo) acupuncture for treatment of nausea of pregnancy. ${ }^{172}$ In a subject- and nausea-masked, randomised, controlled trial, 55 women between 6 and 10 weeks' gestation were given genuine, traditional-style acupuncture or sham treatment with a cocktail stick on three or four occasions over 3 weeks. The main outcome measure was nausea score, as determined by subject report on a visual analogue scale in a daily diary. Anxiety and depression were also assessed. Nausea scores decreased from a median of 85.5 (interquartile range 71.25-89.75) to 47.5 (interquartile range 29.25-69.5) in the acupuncture group and from 87.0 (interquartile range 73.0-93.0) to 48.0 (interquartile range 14.0-80.0) in the sham treatment group. There was strong evidence of a time effect $(P<0.001)$ but no evidence of a group effect $(P=0.9)$ or a group-time interaction $(P=0.8)$. Similarly, there was evidence of time effects in 
scores for anxiety and depression but no group differences. The study had a power score of $95 \%$ to detect significant differences in nausea scores. It was concluded that acupuncture is as effective in treating nausea of pregnancy as a sham procedure.

\subsection{Neck pain (2 systematic reviews/1 clinical trial)}

Minimal acupuncture is easily incorporated into primary care consultations, but there is no rigorous evidence for its effectiveness. In a cohort study, ${ }^{173}$ minimal acupuncture was given to 32 patients with acute neck pain. Neck pain scores, measured by the Northwick Park Neck Pain questionnaire, fell from an average of $12.1( \pm 5.4)$ before treatment to $4.8( \pm 5.6)$ at 3 months $(p<0.001)$. Three months after acupuncture treatment $76 \%$ of patients reported themselves "much better". Out of 18 patients who had had pain lasting less than 2 weeks, 16 required only one treatment. These results suggest that minimal acupuncture may be an effective treatment for neck pain and further definitive studies are recommended.

The objective of another systematic review ${ }^{174}$ was to establish whether there is evidence for or against the efficacy of acupuncture in the treatment of neck pain. Two reviewers independently extracted data concerning study methods, quality and outcome. Overall, the outcomes of 14 randomised clinical trials were equally balanced between positive and negative. Acupuncture was superior to waiting list in one study, and either equal or superior to physiotherapy in three studies. Needle acupuncture was not superior to indistinguishable sham control in four out of five studies. Of the eight high-quality trials, five were negative. Therefore, the hypothesis that acupuncture is efficacious in the treatment of neck pain is not supported by the available evidence from sound clinical trials. Further studies are required.

Chiropractic spinal manipulation (CSM) is often used as a treatment for neck pain. However, its effectiveness is unclear. The aim of this article ${ }^{175}$ was to evaluate systematically and critically the effectiveness of CSM for neck pain. Six electronic databases were searched for all relevant randomised clinical trials. Strict inclusion/exclusion criteria had been predefined. Key data were validated and extracted. Methodological quality was assessed using the Jadad score. Statistical pooling was anticipated but was deemed not feasible. Four studies met the inclusion/exclusion criteria. Two studies were on single interventions, and 2 included series of CSM treatments, both with 1 12-month follow-up. The 2 short-term trials used spinal mobilisation as a control intervention. The 2 long-term studies compared CSM with exercise therapy. None of the 4 trials convincingly demonstrated the superiority of CSM over control interventions. In conclusion, the notion that CSM is more effective than conventional exercise treatment in the treatment of neck pain was not supported by rigorous trial data.

\subsection{Nicotine addiction (2 systematic reviews/1 clinical trial)}

\subsubsection{Acupuncture}

A randomised, sham-controlled trial of acupuncture for smoking cessation was performed with 2 parallel treatment arms; the participant and the evaluator were unaware of which treatment was received. ${ }^{176}$ Seventy-six adults who wanted to stop smoking received either $100-\mathrm{Hz}$ electroacupuncture with needles inserted into the appropriate point in each ear or a sham control procedure over the mastoid bone. Interventions were given on days 1, 3, and 7 of smoking cessation. Nicotine withdrawal symptoms were measured by visual analogue scale scores recorded in a daily diary for 14 days; smoking cessation was confirmed objectively. There was no significant difference 
between the mean reduction of withdrawal symptom scores of the 2 groups from day 1 to day 14 . Fifteen participants (39\%) who received electroacupuncture and 16 participants $(42 \%)$ who received a sham procedure were abstinent on day 14 . This form of electroacupuncture was therefore no more effective than placebo in reducing nicotine withdrawal symptoms.

The objective of a systematic review ${ }^{177}$ was to determine the effectiveness of acupuncture in smoking cessation in comparison with a) sham acupuncture b) other interventions c) no intervention. We searched ten electronic databases. Randomised clinical trials comparing a form of acupuncture with either sham acupuncture, another intervention or no intervention for smoking cessation were selected. We extracted data in duplicate on the type of subjects, the nature of the acupuncture and control procedures, the outcome measures, method of randomisation, and completeness of follow-up. We assessed abstinence from smoking at the earliest time-point (before 6 weeks), at six months and at one-year follow-up in patients smoking at baseline. We used the most rigorous definition of abstinence for each trial, and biochemically validated rates if available. Those lost to follow-up were counted as continuing to smoke. Where appropriate, we performed meta-analyses using a fixed effects model. We identified 22 studies. Acupuncture was not superior to sham acupuncture in smoking cessation at any time point. The odds ratio (OR) for early outcomes was 1.22 (95\% confidence interval 0.99 to 1.49$)$; the OR after 6 months was 1.50 (95\% confidence interval 0.99 to 2.27 ) and after 12 months 1.08 (95\% confidence interval 0.77 to 1.53 ). Similarly, when acupuncture was compared with other anti-smoking interventions, there were no differences in outcome at any time point. Acupuncture appeared to be superior to no intervention in the early results, but this difference was not sustained. The results with different acupuncture techniques do not show any one particular method (i.e. auricular acupuncture or non-auricular acupuncture) to be superior to control intervention.

\subsubsection{Hypnotherapy}

The objective of a further systematic review ${ }^{178}$ was to evaluate the effectiveness of hypnotherapy as a treatment for smoking cessation. Randomised clinical trials of hypnotherapy for smoking cessation, with reports of smoking status at least six months after the beginning of treatment, were included. The main outcome was abstinence from smoking at 6-12 months. Data were extracted independently by three reviewers. Nine studies were found comparing hypnotherapy with 14 different control interventions. These were grouped into waiting list/no treatment controls (three studies), attention/advice interventions (four studies), psychological treatments (two studies) and rapid or focused smoking (two studies). In three studies, hypnotherapy was used in conjunction with counselling and compared with counselling alone. Due to significant heterogeneity in the results, pooled odds ratios were not calculated for waiting list, attention/advice or counselling alone comparisons. The studies varied in the type of hypnotic induction used and the duration of the hypnosis session. The number of sessions also varied greatly (from one to nine) and the total duration of hypnosis administered during treatment varied from 80 minutes to seven hours. Consequently it was not possible to categorise the studies on the basis of type and duration of hypnosis. There was insufficient evidence to determine whether hypnotherapy was better than either no treatment or any of the alternative interventions. Thus hypnotherapy has not been shown to have a greater effect on six month quit rates than other interventions or no treatment. The effects of hypnotherapy on smoking cessation claimed by uncontrolled studies were not confirmed by analysis of randomised clinical trials.

\subsection{Non-ulcer dyspepsia (1 systematic review)}

Our aim was to critically assess the evidence for and against herbal medicinal products for the treatment of non-ulcer dyspepsia. ${ }^{179}$ Systematic searches were performed in six electronic databases 
and the reference lists located were checked for further relevant publications. No language restrictions were imposed. Experts in the field and manufacturers of identified herbal extracts were also contacted. All randomised clinical trials of herbal medicinal products administered as supplements to human subjects were included. Seventeen randomised clinical trials were identified, nine of which involved peppermint and caraway as constituents of combination preparations. Symptoms were reduced by all treatments $(60-95 \%$ of patients reported improvements in symptoms). The mechanism of any anti-dyspeptic action is difficult to define, as the causes of nonulcer dyspepsia are unclear. There appear to be few adverse effects associated with these remedies, although, in many cases, comprehensive safety data were not available. We concluded that there are several herbal medicinal products with anti-dyspeptic activity and encouraging safety profiles. Further research is warranted to establish their therapeutic value in the treatment of non-ulcer dyspepsia.

\subsection{Osteoarthritis (1 clinical trial/7 systematic reviews)}

\subsubsection{CAM general}

Complementary or alternative therapies for osteoarthritis are commonly used and therefore it is important that health-care providers and patients are aware of the evidence for or against these approaches. In this article, ${ }^{180}$ the best available evidence is reviewed. The results suggest that, for several treatments, the risk-benefit profile is encouraging: acupuncture, several herbal medicines and capsaicin cream. For other therapies the evidence is weak or contradictory: homeopathy, magnet therapy, tai chi, leech therapy, music therapy, yoga, imagery and therapeutic touch. Many other treatments have not been scientifically tested. It is concluded that some complementary or alternative therapies have generated sufficiently promising results to warrant further investigation in large-scale, definitive, randomized clinical trials

\subsubsection{Magnets}

The objective of this study ${ }^{181}$ was to determine the effectiveness of commercially available magnetic bracelets for pain control in osteoarthritis of the hip and knee.194 men and women aged 45-80 years with osteoarthritis of the hip or knee were included in this RCT. Three patient groups were treated either with a standard strength static bipolar magnetic bracelet, a weak magnetic bracelet, or a non-magnetic (dummy) bracelet for 12 weeks. The main outcome measures were change in the Western Ontario and McMaster Universities osteoarthritis lower limb pain scale (WOMAC A) after 12 weeks, with the primary comparison between the standard and dummy groups. Secondary outcomes included changes in WOMAC B and C scales and a visual analogue scale for pain. Mean pain scores were reduced more in the standard magnet group than in the dummy group (mean difference 1.3 points, $95 \%$ confidence interval 0.05 to 2.55 ). Self reported blinding status did not affect the results. The scores for secondary outcome measures were consistent with the WOMAC A scores. We concluded that pain from osteoarthritis of the hip and knee decreases when wearing magnetic bracelets. It is uncertain whether this response is due to specific or non-specific (placebo) effects.

\subsubsection{Acupuncture}

Acupuncture is a popular complementary treatment for osteoarthritis. In order to define its effectiveness, a systematic review of the literature was undertaken. ${ }^{182}$ Independent literature searches identified eleven studies of acupuncture for osteoarthritis. Their results are highly contradictory. Most trials suffer from methodological flaws. The most rigorous studies suggest that 
acupuncture is not superior to sham needling in reducing pain of osteoarthritis: both alleviate symptoms to approximately the same degree. This could either mean sham needling has similar specific effects as acupuncture or that both methods are associated with considerable non-specific effects. Future research should clarify which explanation applies

An update of this systematic review evaluates the evidence for the effectiveness of acupuncture in peripheral joint osteoarthritis (OA). ${ }^{183}$ Systematic searches were conducted on Medline, Embase, AMED, Cochrane Library, CINAHL, British Nursing Index, PsychINFO and CAMPAIN until July 2005. Hand-searches included conference proceedings and our own files. There were no restrictions regarding the language of publication. All randomized controlled trials (RCTs) of acupuncture for patients with peripheral joint OA were considered for inclusion. Trials assessing needle acupuncture with or without electrical stimulation were considered if sham- or placebo-controlled or controlled against a comparator intervention. Trials testing other forms of acupuncture were excluded. Methodological quality was assessed and, where possible, meta-analyses were performed. Thirtyone possibly relevant studies were identified and 18 RCTs were included. Ten trials tested manual acupuncture and eight trials tested electro-acupuncture. Overall, ten studies demonstrated greater pain reduction in acupuncture groups compared with controls. The meta-analysis of homogeneous data showed a significant effect of manual acupuncture compared with sham acupuncture (standardized mean difference $0.24,95 \%$ confidence interval $0.01-0.47, \mathrm{P}=0.04, \mathrm{n}=329$ ), which is supported by data for knee OA. The extent of heterogeneity in trials of electro-acupuncture prevented a meaningful meta-analysis. Sham-controlled RCTs suggest specific effects of acupuncture for pain control in patients with peripheral joint OA. Considering its favourable safety profile acupuncture seems an option worthy of consideration particularly for knee OA. Further studies are required particularly for manual or electro-acupuncture in hip OA.

\subsubsection{Homeopathy}

Osteoarthritis is a common rheumatic disease which is often treated with complementary therapies. Limitations of conventional medical management of this condition indicate a real need for safe and effective treatment of osteoarthritic patients. We systematically reviewed the clinical evidence for or against the effectiveness of homeopathic medicines in the treatment of patients with osteoarthritis. ${ }^{184}$ A comprehensive search yielded four randomised clinical trials. We conclude that the small number of randomised clinical trials conducted to date, although favouring homeopathic treatment, do not allow a firm conclusion as to the effectiveness of homeopathic remedies in the treatment of patients with osteoarthritis. The clinical evidence appears promising, however, and more research into this area seems warranted.

\subsection{5 $\underline{\mathrm{ASU}}$}

Another systematic review was aimed at critically evaluating the data from rigorous clinical trials of ASU. ${ }^{185}$ Six databases were searched for randomised, placebo-controlled, double-blind trials of ASU. Four studies could be included. Key data were extracted according to pre-defined criteria and tabulated as well as discussed in narrative form. The methodological quality of the four studies was high. They included patients with OA of the knee and hip. The dose of ASU was $300 \mathrm{mg} /$ day and one trial also included a group treated with $600 \mathrm{mg} /$ day. Three of the four trials suggested efficacy of ASU for improving symptoms of OA. The majority of rigorous trial data available to date suggest that ASU is effective for the symptomatic treatment of OA and more research seems warranted. However, the only real long-term trial yielded a largely negative result. 


\subsubsection{Supplements}

The objectives of this systematic review ${ }^{186}$ were to critically evaluate the evidence regarding complementary and alternative medicine (CAM) taken orally or applied topically (excluding glucosamine and chondroitin) in the treatment of OA. Randomized clinical trials of OA using CAMs, in comparison with other treatments or placebo, published in English up to January 2009, were eligible for inclusion. They were identified using systematic searches of bibliographic databases and manual searching of reference lists. Information was extracted on outcomes, and statistical significance, in comparison with alternative treatment of placebo, and side effects were reported. The methodological quality of the primary studies was determined. The present review found consistent evidence that capsaicin gel and S-adenosyl methionine were effective in the management of OA. There was also some consistency to the evidence that Indian Frankincense, methylsulphonylmethane and rose hip may be effective. For other substances with promising evidence, the evidence base was either insufficiently large or the evidence base was inconsistent. Most of the CAM compounds studied were free of major adverse effects. In conclusion, the major limitation in reviewing the evidence is the paucity of randomized controlled trials in the area: widening the evidence base, particularly for those compounds for which there is promising evidence, should be a priority for both researchers and funders

\subsubsection{Tai chi}

The aim of this systematic review ${ }^{187}$ was to evaluate data from controlled clinical trials testing the effectiveness of tai chi for treating osteoarthritis. Systematic searches were conducted on MEDLINE, AMED, British Nursing Index, CINAHL, EMBASE, PsycInfo, The Cochrane Library 2007, Issue 2, the UK National Research Register and ClinicalTrials.gov, Korean medical databases, the Qigong and Energy database and Chinese medical databases (until June 2007). Hand searches included conference proceedings and our own files. There were no restrictions regarding the language of publication. All controlled trials of tai chi for patients with osteoarthritis were considered for inclusion. Methodological quality was assessed using the Jadad score. Five randomised clinical trials (RCTs) and seven non-randomised controlled clinical trials (CCTs) met all inclusion criteria. Five RCTs assessed the effectiveness of tai chi on pain of osteoarthritis (OA). Two RCTs suggested significant pain reduction on visual analog scale or Western Ontario and McMaster Universities Osteoarthritis Index (WOMAC) compared to routine treatment and an attention control program in knee OA. Three RCTs did not report significant pain reduction on multiple sites pain. Four RCTs tested tai chi for physical functions. Two of these RCTs suggested improvement of physical function on activity of daily living or WOMAC compared to routine treatment or wait-list control, whilst two other RCTs failed to do so. In conclusion, there is some encouraging evidence suggesting that tai chi may be effective for pain control in patients with knee OA. However, the evidence is not convincing for pain reduction or improvement of physical function.

\subsection{Osteoporosis (2 systematic reviews/5 clinical trials)}

\subsection{1 $\underline{\text { Exercise }}$}

The aim of this systematic review ${ }^{188}$ was to determine whether exercise can be useful in the prevention of osteoporosis. Various literature searches identified 21 randomised clinical trials on the subject. These are highly diverse in more than one respect. Collectively, however, they strongly suggest that regular physical exercise can reduce the risk of osteoporosis and delay the physiological 
decrease of bone mineral density. It was concluded that regular exercise for women of practically all ages is well advised.

\subsection{2 $\underline{\text { Tai chi }}$}

Tai chi may have beneficial effects with respect to balance, falls and non-vertebral fractures. The purpose of this systematic review ${ }^{189}$ was to evaluate evidence from controlled clinical trials testing the effectiveness of tai chi for osteoporosis. Systematic searches were conducted on 20 electronic databases. The outcome measures considered for inclusion were changes in bone parameters. Five randomized clinical trials (RCTs) and two controlled clinical trials (CCT) met all inclusion criteria. In postmenopausal women, one RCT found tai chi to be superior for loss of bone mineral density (BMD) compared with sedentary lifestyle, while two other RCTs found no differences between tai chi and exercises or calcium supplementation for BMD. The meta-analysis showed no significant effect of tai chi on BMD change at the spine compared with no treatment in postmenopausal women. One RCT failed to show favorable effects of tai chi compared with resistance training (RT) for total hip BMD in elderly women. A further RCT compared tai chi with RT on bone metabolism and reported favorable effects compared with RT in the elderly.

Five controlled trials with follow-up periods of 1, 2, 3, 4 and 5 years were conducted to evaluate the influence of a therapeutic exercise regimen on forearm bone density in postmenopausal women. ${ }^{190}$ Between 1986 and 1990, all postmenopausal women presenting with predominantly recurrent back pain were randomly assigned to either a control or an exercise group and were followed up for bone density for up to 5 years. Patients who did not show up for follow-up tests, or who started drug treatment for osteoporosis were retrospectively excluded from the analysis. According to the women's compliance, the exercise group was further divided into 2 subgroups: exercise group A (regular exercise), and exercise group B (irregular exercise). There was no significant change in forearm bone density observed in exercise group A regardless of the length of follow-up period, whereas bone density decreased significantly $(p<0.05)$ in the control group after $1,2,3$ and 4 years, and in exercise group B after 4 and 5 years of follow-up. We concluded therefore that regular therapeutic exercises may delay the loss of forearm bone mass in postmenopausal women.

\subsection{Otitis (1 systematic review)}

In an attempt to find all clinical trials on this subject, I conducted electronic literature ${ }^{191}$ searches in the following databases: Medline, Embase, Cinhal and AMED (September 2008). No language or time restrictions were imposed. To get included, an article needed to refer to a controlled clinical trial of chiropractic for ear infection (otitis). Case reports, case series and uncontrolled or feasibility studies were excluded. These searches generated 35 hits. After removing duplicates, 27 articles were read. None of them met the inclusion criteria. Previous research has shown that professional chiropractic organisations 'make claims for the clinical art of chiropractic that are not currently available scientific evidence...'. The claim to effectively treat otitis seems to be one of them. It is time now, I think, that chiropractors either produce the evidence or abandon the claim.

\subsection{Other pain syndromes (13 systematic reviews/2 clinical trials/ 1 case report)}

\subsubsection{General}

The objective of this systematic review ${ }^{192}$ was to assess the evidence from rigorous clinical trials, systematic reviews, and meta-analyses of complementary and alternative therapies for treating 
neuropathic and neuralgic pain. Systematic searches were carried out in the databases Medline, Embase, Amed, Scopus, the Cochrane Database of Systematic Reviews, Natural Standard, and the Natural Medicines Comprehensive Database. Each database was searched from its respective inception until March 2006. To be included, trials were required to state that they were randomized. Systematic reviews and meta-analyses were included if based on the results of randomized trials. No language restrictions were imposed. Five relevant systematic reviews and meta-analyses and 15 additional trials met the inclusion criteria and were reviewed. Data on the following complementary and alternative medicine treatments were identified: acupuncture, electrostimulation, herbal medicine, magnets, dietary supplements, imagery, and spiritual healing. Conclusions: On the basis of our findings, the evidence is not fully convincing for most complementary and alternative medicine modalities in relieving neuropathic or neuralgic pain. However, for topically applied capsaicin there is evidence of effectiveness beyond placebo. The evidence can be classified as encouraging and warrants further study for cannabis extract, magnets, carnitine, and electrostimulation.

\subsubsection{Acupuncture}

Acupuncture is also frequently advocated as an effective treatment for dental pain. The question of whether or not it is effective for this indication remains controversial. The aim of this systematic review ${ }^{193}$ therefore was to assess the effectiveness of acupuncture in dental pain. Only controlled trials were included in this review. Information was extracted from included studies and entered on standard forms independently by both authors. Methodological quality was assessed using the Jadad score. 16 such studies were located. The majority of these trials imply that acupuncture is effective in dental analgesia. However, important questions remain unanswered. It was therefore concluded that acupuncture can alleviate dental pain and that future investigations should define the optimal acupuncture technique and its relative efficacy compared with conventional methods of analgesia.

The aim of this systematic review ${ }^{194}$ is to assess the effectiveness of acupuncture as an adjunctive analgesic method to standard anaesthetic procedures for surgery and to determine whether acupuncture has any analgesic-sparing effect. Electronic literature searches for randomized clinical trials (RCTs) of acupuncture during surgery were performed in seven electronic databases. No language restrictions were imposed. All included studies were rated according to their methodological quality and validity. As the studies were clinically heterogeneous, no metaanalyses were performed. The evidence was classified according to four levels: strong, moderate, limited, or inconclusive. Nineteen RCTs were identified. Seven of them suggested that acupuncture is efficacious. Of nine high-quality RCTs, two studies had positive outcomes. There was no significant association between study quality and direction of outcome. One of eight high-validity trials reported a positive outcome and there was a significant relationship between validity and direction of outcome. The evidence that acupuncture is more effective than no acupuncture as an adjunct to standard anaesthetic procedures is therefore inconclusive. Strong evidence exists that real acupuncture is not significantly different from placebo acupuncture. For an analgesic-sparing effect of acupuncture, evidence remains inconclusive. In conclusion, this review does not support the use of acupuncture as an adjunct to standard anaesthetic procedures during surgery.

\subsubsection{Auricular acupuncture}

The number of publications on the peri-operative use of auricular acupuncture has rapidly increased within the last decade. The aim was to evaluate clinical evidence on the efficacy of auricular acupuncture for postoperative pain control. Electronic databases: Medline, MedPilot, DARE, Clinical Resource, Scopus and Biological Abstracts were searched from their inception to September 2007. All randomised clinical trials on the treatment of postoperative pain with auricular 
acupuncture were considered and their quality was evaluated using the Jadad scale. Pain intensity and analgesic requirements were defined as the primary outcome measures. Of 23 articles, nine fulfilled the inclusion criteria. Meta-analytic approach was not possible because of the heterogeneity of the primary studies. In eight of the trials, auricular acupuncture was superior to control conditions. Seven randomised clinical trials scored three or more points on the Jadad scale but none of them reached the maximum of 5 points. The evidence that auricular acupuncture reduces postoperative pain is promising but not compelling. ${ }^{195}$

\subsubsection{Bee venom acupuncture}

Bee venom (BV) acupuncture (BVA) involves injecting diluted BV into acupoints and is used for arthritis, pain, and rheumatoid diseases. The objective of this systematic review ${ }^{196}$ was to evaluate the evidence for the effectiveness of BVA in the treatment of musculoskeletal pain. Seventeen electronic databases were systematically searched up to September 2007 with no language restrictions. All randomized clinical trials (RCTs) of BVA for patients with musculoskeletal pain were considered for inclusion if they included placebo controls or were controlled against a comparator intervention. Methodology quality was assessed and, where possible, statistical pooling of data was performed. A total of 626 possibly relevant articles were identified, of which 11 RCTs met our inclusion criteria. Four RCTs that tested the effects of BVA plus classic acupuncture compared with saline injection plus classic acupuncture were included in the main meta-analysis. Pain was significantly lower with BVA plus classic acupuncture than with saline injection plus classic acupuncture (weighted mean difference: $100-\mathrm{mm}$ visual analog scale, $14.0 \mathrm{~mm}, 95 \% \mathrm{CI}$ 9.5-18.6, P <.001, $\mathrm{n}_{-} 112$; heterogeneity: _2 _ $\left.0,2_{-}{ }_{-} 1.92, \mathrm{P} \_.59, \mathrm{I} 2 \_0 \%\right)$. Our results provide suggestive evidence for the effectiveness of BVA in treating musculoskeletal pain. However, the total number of RCTs included in the analysis and the total sample size were too small to draw definitive conclusions.

\subsubsection{Chiropractic}

Chiropractic manipulation is mostly used for spinal problems but, in an increasing number of cases, also for non-spinal conditions. This systematic review ${ }^{197}$ is aimed at critically evaluating the evidence for or against the effectiveness of this approach. Five electronic databases were searched for all randomised clinical trials of chiropractic manipulation as a treatment of non-spinal pain. They were evaluated according to standardised criteria. Eight such studies were identified. They related to the following conditions: fibromyalgia, carpal tunnel syndrome, infantile colic, otitis media, dysmenorrhea and chronic pelvic pain. Their methodological quality ranged from mostly poor to excellent. Their findings do not demonstrate that chiropractic manipulation is an effective therapy for any of these conditions. It was concluded that only very few randomised clinical trials exist of chiropractic manipulation as a treatment of non-spinal conditions. The claim that this approach is effective for such conditions is not based on data from rigorous clinical trials.

\subsubsection{Cupping}

The objective of this study ${ }^{198}$ was to assess the evidence for or against the effectiveness of cupping as a treatment option for pain. Fourteen databases were searched. Randomized clinical trials (RCTs) testing cupping in patients with pain of any origin were considered. Trials using cupping with or without drawing blood were included, while trials comparing cupping with other treatments of unproven efficacy were excluded. Trials with cupping as concomitant treatment together with other treatments of unproven efficacy were excluded. Trials were also excluded if pain was not a central symptom of the condition. The selection of studies, data extraction and validation were performed independently by three reviewers. Seven RCTs met all the inclusion criteria. Two RCTs suggested 
significant pain reduction for cupping in low back pain compared with usual care (P50.01) and analgesia (P50.001). Another two RCTs also showed positive effects of cupping in cancer pain (P50.05) and trigeminal neuralgia (P50.01) compared with anticancer drugs and analgesics, respectively. Two RCTs reported favorable effects of cupping on pain in brachialgia compared with usual care ( $\mathrm{P} 1 / 40.03)$ or heat pad (P50.001). The other RCT failed to show superior effects of cupping on pain in herpes zoster compared with antiviral medication $\left(\mathrm{P}^{1} / 40.065\right)$. Currently there are few RCTs testing the effectiveness of cupping in the management of pain. Most of the existing trials are of poor quality. Therefore, more rigorous studies are required before the effectiveness of cupping for the treatment of pain can be determined.

\subsubsection{Homoeopathy}

Homoeopathic arnica is widely believed to control bruising, reduce swelling and promote recovery after local trauma; many patients therefore take it perioperatively. To determine whether this treatment has any effect, we conducted a double-blind, placebo-controlled, randomised trial with three parallel arms. ${ }^{199}$ Sixty-four adults undergoing elective surgery for carpal tunnel syndrome were randomised to take three tablets daily of homoeopathic arnica $30 \mathrm{C}$ or $6 \mathrm{C}$ or placebo for seven days before surgery and fourteen days after surgery. Primary outcome measures were pain (short form McGill Pain Questionnaire) and bruising (colour separation analysis) at four days after surgery. Secondary outcome measures were swelling (wrist circumference) and use of analgesic medication (patient diary). Sixty-two patients could be included in the intention-to-treat analysis. There were no group differences on the primary outcome measures of pain $(P=0.79)$ and bruising $(P=0.45)$ at day four. Swelling and use of analgesic medication also did not differ between arnica and placebo groups. Adverse events were reported by 2 patients in the arnica $6 \mathrm{C}$ group, 3 in the placebo group and 4 in the arnica $30 \mathrm{C}$ group. The results of this trial do not suggest that homoeopathic arnica has an advantage over placebo in reducing postoperative pain, bruising and swelling in patients undergoing elective hand surgery.

Following the publication of this trial, 16 case reports of apparently beneficial effects of Arnica came to our attention. ${ }^{200}$ Many of these apparent responses could have been due to other factors including the use of herbal (non-diluted) Arnica, placebo response and natural course of disease.

\subsubsection{Autogenic training}

The aim of this investigation was to test the benefit of autogenic training in patients with reflex sympathetic dystrophy. ${ }^{201}$ Eighteen patients with reflex sympathetic dystrophy of the upper limb were recruited and randomised in Group A (autogenic training) and C (controls). All patients received home therapy, including elevation of the affected extremity, therapeutic exercise and cryotherapy. In Group A, additional autogenic training was applied once a week for ten weeks.

Before and at completion of this treatment period, patients underwent a clinical examination comprising measurements of limb volume, skin temperature and range of motion. In addition, pain was assessed by means of a visual analogue scale. The results show beneficial changes in both groups related to pain, range of motion and limb volume. Yet cross-sectional comparisons revealed no differences in these parameters. Only the changes in skin temperature showed significant intergroup differences. It is concluded that autogenic training may be helpful in certain aspects of reflex sympathetic dystrophy but its potential value requires further study.

\subsubsection{Magnets}

Static magnets are marketed with claims of effectiveness for reducing pain, although evidence of scientific principles or biological mechanisms to support such claims is limited. We performed a 
systematic review ${ }^{202}$ and meta-analysis to assess the clinical evidence from randomized trials of static magnets for treating pain. Systematic literature searches were conducted from inception to March 2007 for the following data sources: MEDLINE, EMBASE, AMED (Allied and Complementary Medicine Database), CINAHL, Scopus, the Cochrane Library and the UK National Research Register. All randomized clinical trials of static magnets for treating pain from any cause were considered. Trials were included only if they involved a placebo control or a weak magnet as the control, with pain as an outcome measure. The mean change in pain, as measured on a $100-\mathrm{mm}$ visual analogue scale, was defined as the primary outcome and was used to assess the difference between static magnets and placebo. Twenty-nine potentially relevant trials were identified. Nine randomized placebo-controlled trials assessing pain with a visual analogue scale were included in the main meta-analysis; analysis of these trials suggested no significant difference in pain reduction (weighted mean difference [on a 100-mm visual analogue scale] $2.1 \mathrm{~mm}, 95 \%$ confidence interval 1.8 to $5.9 \mathrm{~mm}, \mathrm{p}=0.29)$. This result was corroborated by sensitivity analyses excluding trials of acute effects and conditions other than musculoskeletal conditions. Analysis of trials that assessed pain with different scales suggested significant heterogeneity among the trials, which means that pooling these data is unreliable. The evidence does not support the use of static magnets for pain relief, and therefore magnets cannot be recommended as an effective treatment. For osteoarthritis, the evidence is insufficient to exclude a clinically important benefit, which creates an opportunity for further investigation.

\subsubsection{Moxibustion}

The objective of this study ${ }^{203}$ was to assess the evidence for or against the effectiveness of moxibustion as a treatment option for pain. Fourteen electronic databases were searched. Randomized clinical trials (RCTs) testing moxibustion in human patients with pain of any type were considered. Trials using direct or indirect moxibustion were included. Studies comparing moxibustion with other treatments of unproven effectiveness, studies testing moxibustion together with other treatments of unproven efficacy and trials where pain was not a central symptom of the condition were all excluded. The selection of studies, data extraction, and validation were performed independently by two reviewers. Four RCTs met all the inclusion criteria. Others were of poor methodological quality. Two RCTs suggested significant pain reductions for indirect moxibustion in osteoarthritis as compared with drug therapy $(\mathrm{n}=200, \mathrm{RR}, 1.11 ; 95 \% \mathrm{CI}$ of 1.02 to $1.21, \mathrm{p}=0.02$, heterogeneity: chi $2=1.03, \mathrm{p}=0.31, \mathrm{I} 2=3 \%$ ). The other two RCTs suggested positive effects of indirect or direct moxibustion on pain in scleroma or herpes zoster compared to drug therapy, respectively. We conclude that few RCTs are available that test the effectiveness of moxibustion in the management of pain, and most of the existing trials have a high risk of bias. Therefore, more rigorous studies are required before the effectiveness of moxibustion for the treatment of pain can be determined.

\subsubsection{Osteopathy}

The objective of this systematic review ${ }^{204}$ was to assess the effectiveness of osteopathy as a treatment option for musculoskeletal pain. Six databases were searched from their inception to August 2010. Only randomized clinical trials (RCTs) were considered if they tested osteopathic manipulation/mobilization against any control intervention or no therapy in human with any musculoskeletal pain in any anatomical location, and if they assessed pain as an outcome measure. The selection of studies, data extraction, and validation were performed independently by two reviewers. Studies of chiropractic manipulations were excluded. Sixteen RCTs met the inclusion criteria. Their methodological quality ranged between 1 and 4 on the Jadad scale (max $=5$ ). Five RCTs suggested that osteopathy compared to various control interventions leads to a significantly 
stronger reduction of musculoskeletal pain. Eleven RCTs indicated that osteopathy compared to controls generates no change in musculoskeletal pain. Collectively, these data fail to produce compelling evidence for the effectiveness of osteopathy as a treatment of musculoskeletal pain.

\subsubsection{Qigong}

The aim of this systematic review ${ }^{205}$ was to assess the clinical evidence of external qigong as a treatment option for pain conditions. Databases were searched up to January 2007. Randomized, clinical trials (RCTs) testing external qigong in patients with pain of any origin assessing clinical outcomes were considered. Trials using any type of control group were included. The selection of studies, data extraction, and validation were performed independently by at least 2 reviewers. One hundred forty-one potentially relevant studies were identified and 5 RCTs could be included. All RCTs of external qigong demonstrated greater pain reductions in the qigong groups compared with control groups. Meta-analysis of 2 RCTs showed a significant effect of external qigong compared with general care for treating chronic pain (Pain $100 \mathrm{~mm}$ VAS; weighted main differences, 36.3 $\mathrm{mm} ; 95 \% \mathrm{CI}, 22.8$ to $49.8 ; P<.001$; heterogeneity: $\left.\_{ }_{2}{ }_{1} 1.79, P_{-} .18, \mathrm{I} 2{ }_{-} 44.0 \%, \mathrm{n} \_80\right)$. The evidence from RCTs testing the effectiveness of external qigong for treating pain is encouraging but not convincing.

The objective of this systematic review ${ }^{206}$ was to assess the evidence for the effectiveness of internal qigong as a treatment option for pain conditions. Nineteen databases were searched through to February 2009. Controlled clinical trials testing internal qigong in patients with pain of any origin assessing clinical outcome measures were considered. Trials using any type of internal qigong and control intervention were included. The selection of studies, data extraction, and validation were performed independently by 2 reviewers. Four randomized clinical trials (RCTs) and 3 controlled clinical trials met all inclusion criteria. One RCT suggested no significant difference for low back pain compared with electromyographic biofeedback. Two RCTs failed to show effects of internal qigong in neck pain compared with exercise therapy and waiting list control. One RCT suggested that qigong is inferior to aerobic exercise in patients with fibromyalgia. There are few RCTs testing the effectiveness of internal qigong in the management of pain conditions. Collectively, the existing trial evidence is not convincing enough to suggest that internal qigong is an effective modality for pain management. This review of controlled clinical trials focused on the effects of internal qigong, a self directed energy healing intervention involving movement and meditation. Collectively, the existing trial evidence is not convincing enough to suggest that internal qigong is an effective modality for pain management. Future studies should be of high quality with particular emphasis on designing an adequate control intervention.

\subsubsection{Spiritual healing}

Spiritual healing is a popular complementary and alternative therapy; in the UK almost 13,000 members are registered in nine separate healing organisations. This randomised clinical trial ${ }^{207}$ was designed to investigate the efficacy of healing in the treatment of chronic pain. One hundred and twenty patients suffering from chronic pain, predominantly of neuropathic and nociceptive origin resistant to conventional treatments, were recruited from a Pain Management Clinic. The trial had two parts: face-to-face healing or stimulated face-to-face healing for $30 \mathrm{~min}$ per week for 8 weeks (part I); and distant healing or no healing for 30 min per week for 8 weeks (part II). The McGill Pain Questionnaire was pre-defined as the primary outcome measure, and sample size was calculated to detect a difference of 8 units on the total pain rating index of this instrument after 8 weeks of healing. VASs for pain, SF36, HAD scale, MYMOP and patient subjective experiences at week 8 were employed as secondary outcome measures. Data from all patients who reached the pre-defined mid-point of 4 weeks ( 50 subjects in part I and 55 subjects in part II) were included in the analysis. 
Two baseline measurements of outcome measures were made, 3 weeks apart, and no significant differences were observed between them. After eight sessions there were significant decreases from baseline in McGill Pain Questionnaire total pain rating index score for both groups in part I and for the control group in part II. However, there were no statistically significant differences between the healing and control groups in either part. In part I the primary outcome measure decreased from 32.8 (95\% CI 28.5-37.0) to 23.3 (16.8-29.7) in the healing group and from 33.1 (27.2-38.9) to 26.1 (19.3$32.9)$ in the simulated healing group. In part II it changed from $29.6(24.8-34.4)$ to 24.0 (18.7-29.4) in the distant healing group and from $31.0(25.8-36.2)$ to $21.0(15.7-26.2)$ in the no healing group. Subjects in healing groups in both parts I and II reported significantly more 'unusual experiences' during the sessions, but the clinical relevance of this is unclear. It was concluded that a specific effect of face-to-face or distant healing on chronic pain could not be demonstrated over eight treatment sessions in these patients.

\subsection{Palliative care (1 review)}

Complementary medicine is fast becoming an integral part of palliative cancer care. There is considerable debate as to whether such treatments require proof of effectiveness through randomised clinical trials or whether it may suffice that patients adopt them with apparent appreciation. In this article, ${ }^{208}$ an attempt was made to raise some of the arguments on both sides. The arguments include the logistical problems of conducting research in palliative care, ethical problems of placebo controls, methodological difficulties of standardising treatments or quantifying subtle effects and to the often considerable costs of clinical trials. The authors felt that neither those who argue against nor those who argue in favour of rigorous clinical trials are entirely wrong or entirely right. However, the final verdict was that an absence of rigorous science will critically hinder progress. This, in turn, would be to the detriment of future patients. Through discussing the strength and weaknesses of both sets of arguments, both sides might learn valuable lessons.

\subsection{Parkinson's disease (3 reviews)}

\subsubsection{Acupuncture}

The objective of this review ${ }^{209}$ is to assess the clinical evidence for or against acupuncture as a treatment for Parkinson disease (PD). We searched the literature using 17 databases from their inception to September 2007 (searched again 3rd January 2008), without language restrictions. We included all randomized clinical trials (RCTs) regardless of their design. Methodological quality was assessed using the Jadad score. Eleven RCTs met all inclusion criteria. Three RCTs assessed the effectiveness of acupuncture on Unified Parkinson's Disease Rating Scale (UPDRS) compared with placebo acupuncture. A meta-analysis of these studies showed no significant effect (n 596 , WMD, 5.7; 95\% CI 22.8 to 14.2, P 5 0.19, heterogeneity: tau2 5 0, v2 5 0.97, P 5 0.62, I2 5 0\%). Another six RCTs compared acupuncture plus conventional drugs on improvement of symptoms of PD with drugs only. A meta-analysis of two of these studies suggested a positive effect of scalp acupuncture (n 5 106, RR, 1.46, 95\% CI 51.15 to 1.87, P 5 0.002; heterogeneity: tau2 50.00 , v2 5 1.14, P 5 0.29, I2 5 12\%). Two further RCTs tested acupuncture versus no treatment. The metaanalysis of these studies also suggested beneficial effects of acupuncture. The results of the latter two types of RCTs fail to adequately control for nonspecific effects. In conclusion, the evidence for the effectiveness of acupuncture for treating PD is not convincing. The number and quality of trials

as well as their total sample size are too low to draw any firm conclusion. Further rigorous trials are warranted. 


\subsubsection{General}

Many people with Parkinson's turn to complementary therapies, despite the fact that there is no complementary therapy that can cure Parkinson's. People have their own individual reasons for this: the therapy may fit in better with their life style, they are disappointed with conventional medical science, they find that it helps to alleviate specific complaints, etc. A book chapter addressed the effects of many complementary therapies on Parkinson's: from acupuncture to aromatherapy, and from massage to yoga. ${ }^{210}$ It was concluded that Parkinson patients should bear the following in mind:

- Use therapists who belong to a credible professional association or society.

- Ensure that there is contact between your regular therapist and your complementary therapists.

- Ask for a clear treatment plan and an estimate of costs.

- Be suspicious of therapists who claim that they can cure Parkinson's or who want to dissociate you from other therapists or carers.

\subsubsection{Tai chi}

The objective of this review ${ }^{211}$ is to assess the effectiveness of tai chi as a treatment option for Parkinson's disease (PD). We have searched the literature using 21 databases from their inceptions to January 2008, without language restrictions. We included all types of clinical studies regardless of their design. Their methodological quality was assessed using the modified Jadad score. Of the seven studies included, one randomised clinical trial (RCT) found tai chi to be superior to conventional exercise in terms of the Unified PD Rating Scale (UPDRS) and prevention of falls. Another RCT found no effects of tai chi on locomotor ability compared with qigong. The third RCT failed to show effects of tai chi on the UPDRS and the PD Questionnaires compared with wait list control. The remaining studies were either non-randomised (n $1 / 41)$ or uncontrolled clinical trials (n $1 / 43$ ). Collectively these data show that RCTs of the tai chi for PD are feasible but scarce. Most investigations suffer from methodological flaws such as inadequate study design, poor reporting of results, small sample size, and publication without appropriate peer review process. In conclusion, the evidence is insufficient to suggest tai chi is an effective intervention for PD. Further research is required to investigate whether there are specific benefits of tai chi for people with PD, such as its potential effect on balance and on the frequency of falls.

\subsection{Pediatric conditions (1 systematic review)}

The use of complementary and alternative medicine (CAM) in paediatric populations is common yet, to date, there has been no synthesis of the evidence of its effectiveness in that population. This overview of systematic reviews ${ }^{212}$ evaluates the evidence for or against the effectiveness of CAM for any childhood condition. Medline, AMED and Cochrane were searched from inception until September 2009. Reference lists of retrieved articles were hand-searched. Experts in the field of CAM were contacted. No language restrictions were applied. 17 systematic reviews were included in this overview, covering acupuncture, chiropractic, herbal medicine, homeopathy, hypnotherapy, massage and yoga. Results were unconvincing for most conditions although there is some evidence to suggest that acupuncture may be effective for postoperative nausea and vomiting, and that hypnotherapy may be effective in reducing procedure-related pain. Most of the reviews failed to mention the incidence of adverse effects of CAMs. Although there is some encouraging evidence for hypnosis, herbal medicine and acupuncture, there is insufficient evidence to suggest that other CAMs are effective for the treatment of childhood conditions. Many of the systematic reviews included in this overview were of low quality, as were the randomised clinical trials within those 
reviews, further reducing the weight of that evidence. Future research in CAM for children should conform to the reporting standards outlined in the CONSORT and PRISMA guidelines.

\subsection{Peripheral vascular disease (4 systematic reviews)}

\subsubsection{Any type of CAM}

While peripheral arterial disease (PAD) affects a considerable proportion of patients in the primary care setting, there is a high level of use of complementary treatment options. The aim was to assess the effectiveness of any type of complementary therapy for peripheral arterial disease. A systematic review was performed. ${ }^{213}$ Literature searches were conducted on Medline, Embase, Amed, and the Cochrane Library until December 2004. Hand-searches of medical journals and bibliographies were conducted. There were no restrictions regarding the language of publication. The screening of studies, selection, data extraction, the assessment of methodologic quality and validation were performed independently by the two reviewers. Data from randomized controlled trials, and systematic reviews and meta-analyses, which based their findings on the results of randomized controlled trials were included. Seven systematic reviews and meta-analyses and three additional randomized controlled trials met the inclusion criteria and were reviewed. The evidence relates to acupuncture, biofeedback, chelation therapy, $\mathrm{CO} 2$-applications and the dietary supplements Allium sativum (garlic), Ginkgo biloba (ginkgo), omega-3 fatty acids, padma 28 and Vitamin E. Most studies included only patients with peripheral arterial disease in Fontaine stage II (intermittent claudication). The reviewed RCTs, systematic reviews and meta-analyses which based their findings on the results of RCTs suggest that G. biloba is effective compared with placebo for patients with intermittent claudication. Evidence also suggests that padma 28 is effective for intermittent claudication, although more data are required to confirm these findings. For all other complementary treatment options there is no evidence beyond reasonable doubt to suggest effectiveness for patients with peripheral arterial disease.

\subsubsection{Herbal medicine}

Ginkgo biloba extract has been reported to have beneficial effects for peripheral vascular disease. We therefore performed a meta-analysis of the efficacy of Ginkgo biloba extract for intermittent claudication based on the results of placebo-controlled, double-blind randomised clinical trials. ${ }^{214}$ Literature searches of the MEDLINE, EMBASE, BIOSIS, AMED, CISCOM, and the Cochrane Library databases were performed to identify studies on the topic. Manufacturers of commercial Ginkgo biloba products and authors of original publications and reviews were contacted to provide additional information. No language restrictions were imposed. Eight randomised, placebocontrolled, double-blind trials were included. The meta-analysis found a significant difference in the increase in pain-free walking distance in favour of Ginkgo biloba (weighted mean difference 34 metres, 95\% confidence interval [CI]: 26 to 43 metres). In studies using similar methodological features (ergometer speed: $3 \mathrm{~km} / \mathrm{h}$, inclination: $12 \%$ ) this difference was 33 metres in favour of Ginkgo biloba (95\% CI: 22 to 43 metres). Adverse effects were rare, mild, and transient. These results therefore suggest that Ginkgo biloba extract is superior to placebo in the symptomatic treatment of intermittent claudication. However, the size of the overall treatment effect is modest and of uncertain clinical relevance. 


\subsubsection{Exercise}

The objective of this systematic review ${ }^{215}$ was to determine whether, in patients with intermittent claudication, an exercise programme was effective in alleviating symptoms and increasing walking distance. Secondary objectives were to determine whether exercise was effective in preventing deterioration of underlying disease and reducing cardiovascular events. All publications describing randomised clinical trials of exercise therapy in intermittent claudication were sought using the search strategy described by the Review Group on Peripheral Vascular Diseases. This strategy included hand searching of relevant medical journals and extensive searches in several electronic databases. All randomised clinical trials of an exercise regimen versus control, or versus medical or surgical therapy, in patients with intermittent claudication due to atherosclerotic disease were included. The exercise regimen was not specified, and inclusion of trials was not affected by the duration, frequency or intensity of the exercise programme. Fourteen trials were identified which met the inclusion criteria, but five were subsequently excluded because of their poor quality. The remaining nine trials involved a total of just over 200 patients. Five trials compared exercise with an untreated control group (three used placebo tablets), two compared exercise with intervention (surgery and angioplasty), and two used drug regimens as the control (antiplatelets and pentoxifylline). The exercise regimens varied, but all recommended at least two sessions weekly.

All trials used a treadmill walking test as one of the outcome measures, six included calf blood flow, and four the ankle brachial pressure index (ABPI). Continuous data were analysed by weighted mean differences, using both the fixed effects and random effects models. Exercise therapy significantly improved maximal walking distance (weighted mean difference (WMD) 6.51 $95 \%$ CI $4.36,8.66$ ), with an overall improvement in walking ability of approximately $150 \%$ (range $74 \%$ to $230 \%$ ). Exercise produced significant improvements in walking distance compared with angioplasty at 12 months (WMD 3.30 mins, 95\% CI 2.21, 4.39), and antiplatelet therapy (WMD 1.06 mins, $95 \%$ CI $0.15,1.97$ ) did not differ significantly from surgical treatment, and was less effective than pentoxifylline (WMD - 0.45 mins, $95 \%$ CI $-0.66,-0.24$ ). Exercise did not have a significant effect on the ABPI or calf blood flow, and these outcomes were similar after angioplasty or antiplatelet therapy. The ABPI was significantly improved following surgery, however, (WMD $0.27,95 \% \mathrm{CI}-0.37,-0.17)$, but there was a higher rate of adverse events (18\%) and deaths experienced by those undergoing surgery. It was concluded that exercise is of significant benefit to patients with intermittent claudication, and therefore should be recommended to all suitable patients.

\subsubsection{Chelation therapy}

For this systematic review ${ }^{216}$ computerised literature searches were performed (MEDLINE 1966 to 1996; CISCOM 1996) to identify all studies on the subjects. Publications thus found were screened for further relevant articles in their bibliographies. In addition, leading experts and national societies $(n=6)$ were contacted and asked for further material. Studies were included in the present review if they related to randomised, placebo-controlled, double-blind clinical trials of intravenous chelation therapy for peripheral arterial occlusive disease (PAOD). Four such publications referring to three trials were found. All studies relate to patients suffering from intermittent claudication. Collectively these data do not show that chelation therapy is an effective treatment for this condition.

\subsection{Postoperative ileus}

Homeopathic remedies are advocated for the treatment of postoperative ileus, yet data from clinical trials are inconclusive. We therefore performed meta-analyses ${ }^{217}$ of existing clinical trials to 
determine whether homeopathic treatment has any greater effect than placebo administration on the restoration of intestinal peristalsis in patients after abdominal or gynecologic surgery. We conducted systematic literature searches to identify relevant clinical trials. Meta-analyses were conducted using RevMan software. Separate meta-analyses were conducted for any homeopathic treatment versus placebo; homeopathic remedies of $<12 \mathrm{C}$ potency versus placebo; homeopathic remedies of $>$ or $=$ $12 \mathrm{C}$ potency versus placebo. A "sensitivity analysis" was performed to test the effect of excluding studies of low methodologic quality. Our endpoint was time to first flatus. Meta-analyses indicated a statistically significant $(\mathrm{p}<0.05)$ weighted mean difference (WMD) in favor of homeopathy (compared with placebo) on the time to first flatus. Meta-analyses of the three studies that compared homeopathic remedies $>$ or $=12 \mathrm{C}$ versus placebo showed no significant difference $(\mathrm{p}>0.05)$. Meta-analyses of studies comparing homeopathic remedies $<12 \mathrm{C}$ with placebo indicated a statistically significant $(\mathrm{p}<0.05)$ WMD in favor of homeopathy on the time to first flatus. Excluding methodologically weak trials did not substantially change any of the results. There is evidence that homeopathic treatment can reduce the duration of ileus after abdominal or gynecologic surgery. However, several caveats preclude a definitive judgment. These results should form the basis of a randomized controlled trial to resolve the issue.

\subsection{Premenstrual syndrome (1 systematic review/1 clinical trial)}

\subsubsection{Any complementary therapy}

Complementary/alternative therapies are popular with women who have premenstrual syndrome. A systematic review was therefore designed to determine whether use of such therapies is supported by evidence of effectiveness from rigorous clinical trials. ${ }^{218}$ Trials were located through searching 7 databases and checking the reference lists of articles. Randomised controlled trials investigating a complementary/alternative therapy in women with premenstrual syndrome published in the peerreviewed literature were included in the review. Twenty-seven trials were included investigating herbal medicine (7 trials), homoeopathy (1), dietary supplements (13), relaxation (1), massage (1), reflexology (1), chiropractic (1), and biofeedback (2). Despite some positive findings, the evidence was not compelling for any of these therapies, with most trials suffering from various methodological limitations. On the basis of current evidence, no complementary/alternative therapy can therefore be recommended as a treatment for premenstrual syndrome.

\subsubsection{St John's wort}

The objective of a pilot study ${ }^{219}$ was to investigate whether St John's wort could relieve symptoms of premenstrual syndrome in a small group of women in order to establish a hypothesis and to test methods for conducting a future randomised clinical trial. Nineteen women with premenstrual syndrome who were in otherwise good physical and mental health and not taking other treatments for premenstrual syndrome participated in this prospective, open, uncontrolled observational study. Volunteers underwent a preliminary screening interview, completed Daily Symptom Ratings for one cycle, and attended a medical screening visit before being diagnosed with premenstrual syndrome. Participants took hypericum tablets for two complete menstrual cycles (1 x $300 \mathrm{mg}$ hypericum extract per day standardised to $900 \mu \mathrm{g}$ hypericin). Symptoms were rated daily throughout the trial using a validated measure. The Hospital Anxiety and Depression scale and modified Social Adjustment Scale were administered at baseline and after one and two cycles of treatment. There were significant reductions in all outcome measures. The degree of improvement in overall premenstrual syndrome scores between baseline and the end of the trial was $51 \%$, with over two thirds of the sample demonstrating at least a 50\% decrease in symptom severity. Tolerance and compliance with the treatment were encouraging. The results of this pilot study suggest that there is 
scope for conducting a randomised, placebo-controlled, double-blind trial to investigate the value of hypericum as a treatment for premenstrual syndrome.

\subsection{Pruritis (2 systematic reviews)}

Uremic pruritus (UP) is a common and bothersome symptom in end-stage renal disease (ESRD) that does not always respond to conventional care. Acupuncture is frequently used for the treatment of a wide range of conditions, but its effects on UP in ESRD patients are unclear. The objective of this review $^{220}$ was to evaluate the effectiveness of acupuncture for UP in patients with ESRD. We searched 16 electronic databases from their inception to November 2009. All prospective clinical studies of needle acupuncture for UP in hemodialysis patients with ESRD were included regardless of their design. Risk of bias of the included studies was assessed using the Cochrane criteria. Three randomized controlled trials and three uncontrolled observational studies were included. All of the included trials reported beneficial effects of acupuncture. However, most of the studies showed high risk of bias, which leaves their reports unconvincing. The current evidence is insufficient to show that acupuncture is an effective treatment for UP in patients with ESRD because of suboptimal quality and lack of methodological rigor of included studies. Future trials should overcome the limitations of the currently available evidence.

The objective of this systematic review ${ }^{221}$ was to determine the efficacy of topical capsaicin in treating pruritus in any medical condition. Data sources were Cochrane library, Medline, Embase, Cinahl and Amed, up to April 2008. No language restrictions. Study selection was absed on randomized, controlled trials comparing topically applied capsaicin with placebo or other standard treatment in patients with pruritus, independently selected by two reviewers. Data were independently extracted by two reviewers. Quality assessed using the Jadad scale. Six randomized controlled trials were identified for inclusion. Three were for hemodialysis-related pruritus and provided insufficient data for the efficacy of topical capsaicin to be evaluated. A crossover study of capsaicin for treating idiopathic intractable pruritus ani reported a statistically significant difference in responder rates favoring capsaicin over placebo for itching scores but included insufficient data for the validity of this result to be assessed. A study on notalgia paresthetica reported a statistically significant difference in the first phase of a crossover study favoring capsaicin over placebo in a visual analogue scale for itch intensity but failed to report data for a second outcome measure. The final study on brachioradial pruritus used an inappropriate design and reported no significant reduction in itch between capsaicin and placebo. In conclusion, there is no convincing evidence for the use of capsaicin to treat pruritus in any medical condition. Further research is needed, and should attempt to address methodological issues identified through this review including unblinding and the suitability of crossover designs.

\subsection{Rheumatic pain (4 reviews)}

An overview addressed the subject of CAM for rheumatic conditions in general. ${ }^{222}$ This article is an attempt to provide an introduction to this subject. Its emphasis is on evaluating the efficacy of CAM treatment modalities. This is achieved by referring to systematic reviews of clinical trials of acupuncture for low back pain, osteoarthritis, fibromyalgia, inflammatory rheumatoid disease and neck pain. Further areas addressed in this way are herbal remedies, fish oil and glucosamine. Moreover, massage therapy and spinal manipulation for back pain are discussed. The final sections of this review deal with the safety and cost of CAM. It is concluded that, in view of the popularity of CAM with rheumatological patients, rigorous research into CAM is the best way forward. 
This review summarises the recent literature on complementary and alternative medicine for rheumatic conditions. ${ }^{223}$ Research has emerged in the following areas: acupuncture, herbal remedies, homoeopathy, magnetic fields, massage therapy, spiritual healing, and supplements. Positive evidence was found in relation to glucosamine, chondroitin, some herbal remedies, and acupuncture. Generally speaking, complementary and alternative medicine is grossly underresearched. Because of the popularity of complementary and alternative medicine, adequately defining risk-benefit relationships is an urgent matter.

\subsubsection{Acupuncture}

Several systematic reviews (SRs) have assessed the effectiveness of acupuncture for rheumatic conditions, often with contradictory conclusions. Our aim is to provide a critical evaluation and summary of these data. ${ }^{224}$ Electronic searches were conducted in 15 databases to locate all SRs on acupuncture for rheumatic conditions published since 2000. Data were extracted by the authors according to pre-defined criteria. We found $30 \mathrm{SRs}$ that met our inclusion criteria. They related to the following rheumatic conditions: FM, low back pain, lateral elbow pain, musculoskeletal pain, orthopaedic diseases, OA, RA, shoulder pain, frozen shoulder, neck disorder, AS and sciatica. Their conclusions were in several instances contradictory. Relatively clear evidence emerged to suggest that acupuncture is effective for OA, low back pain and lateral elbow pain and ineffective for FM and RA. In conclusion, many SRs have recently been done. Only for OA, low back pain and lateral elbow pain is the evidence sufficiently sound to warrant positive recommendations of this therapy in routine care of rheumatic patients

\subsubsection{Herbal medicine}

$\underline{\text { Rheumatic pain is often treated by various herbal remedies (see above). For a systematic review, }{ }^{225}}$ 19 randomised clinical trials of herbal medicines for pain were identified. Collectively, these data point toward a considerable potential of phyto-analgesics in rheumatic conditions. All the herbal medicinal drugs have an effect on the eicosanoid metabolism in inhibiting either both or one of the cyclooxygenase and lipoxygenase pathways. Other pathways might be stimulated by GLA and the combination of Populus, Solidago, and Fraxinus to decrease the anti-inflammatory mediators. However, the true mechanism is still unknown for most herbal extracts.

Phytodolor ${ }^{\circledR}$, a standardised extract of Populus tremula marketed for rheumatic pain, was identified through a thorough literature search as the remedy for which more randomised clinical trials have been conducted as a treatment for rheumatic pain than for any other herbal preparation. Another systematic review ${ }^{226}$ was aimed at assessing its efficacy. Six placebo-controlled randomised clinical trials show that Phytodolor ${ }^{\circledR}$ is superior to placebo. Seven randomised clinical trials conducted against active treatments demonstrate that it is as effective as synthetic drugs. Even though several caveats exist, it was concluded that Phytodolor ${ }^{\circledR}$ is an effective treatment for musculoskeletal pain.

\subsection{Rheumatoid arthritis (5 systematic reviews)}

\subsubsection{Acupuncture}

The aim of this systematic review ${ }^{227}$ is to evaluate the available evidence, from randomized clinical trials (RCTs), of acupuncture for treating patients with RA. Systematic searches were conducted on 17 databases up to April 2008 without the language restriction. All RCTs of acupuncture, with or without electrical stimulation or moxibustion, for patients with RA were considered for inclusion. A total of 236 potentially relevant studies were identified and eight RCTs were included. Four RCTs compared the effects of manual or electro-acupuncture with penetrating or non-penetrating sham 
acupuncture and failed to show specific effects of acupuncture on pain $[\mathrm{n} 1 / 488$; weighted mean differences (WMD), 10cm VAS _0.46; 95\% CI_1.70, 0.77; P1/40.46; heterogeneity:_21/40.19; _21/42.38; P1/40.30; I 21/416\%] or other outcome measures. One RCT compared manual acupuncture with indomethacin and suggested favourable effects of acupuncture in terms of total response rate. Three RCTs tested acupuncture combined with moxibustion, vs conventional drugs and failed to show that acupuncture plus moxibustion was superior to conventional drugs in terms of response rate $\left(\mathrm{n}^{1 / 4345}\right.$; RR 1.12; 95\% CI 0.99, 1.28; P1/40.08; heterogeneity:_21/40.00; 221/41.34; $\mathrm{P} 1 / 40.51 ; \mathrm{I}$ $2 \frac{1}{4} 0 \%$ ), pain reduction ( $\mathrm{n}^{1 / 4105}$; WMD, $10 \mathrm{~cm}$ VAS 1.53; 95\% CI_0.57, 3.63; P1/40.15; heterogeneity: 221/41.18; 2 21/41.81; $\mathrm{P}^{1 / 40.18} ; \mathrm{I}_{2}^{1 / 445 \%)}$ or joint swelling index $\left(\mathrm{n}^{1 / 4} 105 ; \mathrm{WMD}, 10 \mathrm{~cm}\right.$ VAS 0.25; 95\% CI_1.31, 1.82; P1/40.75; heterogeneity:_21/40.18;21/41.14; P1/40.28; I 21/413\%). In conclusion, penetrating or non-penetrating sham-controlled RCTs failed to show specific effects of acupuncture for pain control in patients with RA. More rigorous research seems to be warranted.

\subsubsection{Herbal medicine}

With the growing interest in herbal therapies among people with rheumatoid arthritis, there exists a need for investigation into their safety and efficacy. The purpose of this systematic review ${ }^{228}$ was to examine the evidence for the use of herbal medicines for rheumatoid arthritis (RA) based on randomised clinical trials (RCTs). A computerised search of eight electronic databases and the bibliographies of identified articles resulted in 14 studies meeting the inclusion criteria. Two raters independently extracted data and rated the trials for quality. There is moderate support for $\gamma-$ linolenic acid (GLA), which is found in some herbal medicines, for reducing pain, tender joint count and stiffness. For other herbal medicines there was only a single RCT available, resulting in weak evidence. In general, herbal preparations were relatively safe to use.

\subsubsection{Ayurvedic treatments}

The objective of this systematic review ${ }^{229}$ was to systematically review all randomized controlled trials (RCTs) on the effectiveness of Ayurvedic medicine for rheumatoid arthritis (RA). Computerized literature searches were performed for all RCTs of Ayurvedic medicine for RA in the following databases: Medline (March 1969 to March 2003), Embase (February 1985 to February 2003), AMED (March 1980 to March 2003), Cochrane Controlled Trial Register (October 1997 to March 2003), and the abstract service of Central Council for Research in Ayurveda and Siddha (CCRAS; 1976 to March 2003). Hand searches were performed in 1 Sri Lankan and 3 Indian journals and the authors' personal files. Key data of included studies were extracted and reviewed. The methodological quality of all studies was evaluated with the Jadad scale. Seven studies met our inclusion criteria. Trials tested either Ayurvedic medicine against placebo or other Ayurvedic medicines. In general, patient and physician global assessments on the severity of pain, and morning stiffness were used as endpoints. Of 3 placebo-controlled RCTs, 1 high-quality trial did not show benefit of the active treatment against placebo, while another incompletely reported study indicated beneficial effects of an Ayurvedic medicine. A further incompletely reported study showed no significant difference. The remaining 4 trials were difficult to interpret because they tested an Ayurvedic medicine against other Ayurvedic medicines whose effects were not proven. We concluded that there is a paucity of RCTs of Ayurvedic medicines for RA. The existing RCTs fail to show convincingly that such treatments are effective therapeutic options for RA.

\subsubsection{Tai chi}

The objective of this systematic review ${ }^{230}$ is to evaluate data from controlled clinical trials testing the effectiveness of tai chi for treating rheumatoid arthritis (RA). Systematic searches were conducted on Medline, Pubmed, AMED, British Nursing Index, CINAHL, EMBASE, PsycInfo, 
The Cochrane Library 2007, Issue 1, the UK National Research Register and ClinicalTrials.gov, Korean medical databases, Qigong and Energy Medicine Database and Chinese databases up to January 2007. Hand-searches included conference proceedings and our own files. There were no restrictions regarding the language of publication. All controlled trials of tai chi for patients with RA were considered for inclusion. Methodological quality was assessed using the Jadad score. The searches identified 45 potentially relevant studies. Two randomized clinical trials (RCTs) and three non-randomized controlled clinical trials (CCTs) met all inclusion criteria. The included RCTs reported some positive findings for tai chi on disability index, quality of life, depression and mood for RA patients. Two RCTs assessed pain outcomes and did not demonstrate effectiveness on pain reduction compared with education plus stretching exercise and usual activity control. The extent of heterogeneity in these RCTs prevented a meaningful meta-analysis. Currently there are few trials testing the effectiveness of tai chi in the management of RA. The studies that are available are of low methodological quality. Collectively this evidence is not convincing enough to suggest that tai chi is an effective treatment for RA. The value of tai chi for this indication therefore remains unproven.

\subsubsection{Thunder god vine}

Tripterygium wilfordii is a Chinese herb with immunosuppressive effects and an established history of use in the treatment of rheumatoid arthritis (RA). We have carried out a systematic review ${ }^{231}$ of randomised clinical trials (RCTs) which assess the effectiveness of $T$. wilfordii in this indication. We included only randomised and controlled studies which tested the effectiveness of $T$. wilfordii monopreparations in the treatment of RA. Studies in any language were included. A search of five electronic databases from inception to February 2005 identified 18 articles which could potentially meet our inclusion criteria. Only 16 of these could be retrieved from the scientific literature and after reading these in full, only two unique RCTs meeting our inclusion criteria were identified. Both indicated that $T$. wilfordii has beneficial effects on the symptoms of RA. However, the literature indicates that $T$. wilfordii is associated with serious adverse events which make the riskbenefit analysis for this herb unfavourable. Therefore, we cannot recommend its use.

\subsection{Rhinosinusitis (1 systematic review)}

This systematic review ${ }^{232}$ assesses the efficacy of herbal medicines for treating rhinosinusitis. Five electronic databases, bibliographies of located papers, manufacturers, and experts in the field. Inclusion of randomized clinical trials (RCT) testing any herbal medicine in rhinosinusitis, as sole or adjunctive treatment. Data were extracted independently by two reviewers following a predetermined protocol. Ten RCTs, testing six different herbal products against placebo (8 RCTs) or "no additional treatment" (2 RCTs) were included. Four RCTs tested Sinupret as adjunctive treatment for either acute ( 3 RCTs) or chronic (1 RCT) rhinosinusitis. The quality of these studies varied, but two in acute sinusitis, including the largest and best quality study, and one in chronic sinusitis reported significant positive findings. Three RCTs tested bromelain in either acute sinusitis (2 RCTs) or patients of mixed diagnosis (chronic and acute sinusitis), and all reported some positive findings. Metanalysis of the two RCTs in acute sinusitis suggested that adjunctive use of bromelain significantly improves some symptoms of acute rhinosinusitis. Single RCTs were identified for 4 other herbal products (Esberitox, Myrtol, Cineole, and Bi Yuan Shu) as treatments for sinusitis, all reported some positive results. The median methodological quality score was 3 of 5 . Evidence that any herbal medicines are beneficial in the treatment of rhinosinusitis is limited, particularly in chronic rhinosinusitis. There is encouraging evidence that Sinupret and bromelain may be effective adjunctive treatments in acute rhinosinusitis. Positive results from isolated RCTs of four other herbal products require independent replication. 


\subsection{Schizophrenia (1 systematic review)}

Acupuncture is sometimes used as a treatment for schizophrenia. The objective of this review ${ }^{233}$ is to assess systematically the clinical evidence for or against acupuncture as a treatment for schizophrenia. Methods: We searched 20 databases from their inception to May 2009 without language restrictions. All randomised clinical trials (RCTs) of acupuncture, with or without electrical stimulation or moxibustion for patients with schizophrenia were considered for inclusion. Results: Thirteen RCTs, all originating from China, met the inclusion criteria. One RCT reported significant effects of electroacupuncture (EA) plus drug therapy for improving auditory hallucunations and positive symptom compared with sham EA plus drug therapy. Four RCTs showed significant effects of acupuncture for response rate compared with antipsychotic drugs $[\mathrm{n}=$ 360 , relative risk $(\mathrm{RR}): 1.18,95 \%$ confidence interval $(\mathrm{CI}): 1.03-1.34, \mathrm{p}=0.01$; heterogeneity: $\mathrm{s} 2=$ $0.00, \mathrm{v} 2=2.98, \mathrm{p}=0.39, \mathrm{I} 2=0 \%]$. Seven RCTs showed significant effects of acupuncture plus antipsychotic drug therapy for response rate compared with antipsychotic drug therapy $(\mathrm{n}=457$, RR: $1.15,95 \%$ CI: $1.04-1.28, \mathrm{p}=0.008$, heterogeneity: $\mathrm{s} 2=0.00, \mathrm{v} 2=6.56, \mathrm{p}=0.36, \mathrm{I} 2=9 \%$ ). Two RCTs tested laser acupuncture against sham laser acupuncture. One RCT found beneficial effects of laser acupuncture on hallucination and the other RCT showed significant effects of laser acupuncture on response rate, Brief Psychiatric Rating Scale and clinical global index compared with sham laser. The methodological quality was generally poor and there was not a single high quality trial. Conclusion: These results provide limited evidence for the effectiveness of acupuncture in treating the symptoms of schizophrenia. However, the total number of RCTs, the total sample size and the methodological quality were too low to draw firm conclusions. As all studies originated from China, international studies are needed to test whether there is any effect.

\subsection{Snoring (1 clinical trial)}

Snoring is not merely a common nuisance but has been identified as a risk factor for poor health. Last resort treatments for palate-based snoring are surgical - reducing the amount of soft palate and/or stiffening it by causing scarring. They carry a burden of risk and expense and have a high recurrence rate. This pilot study ${ }^{234}$ was a first step in determining whether singing exercises could be used as a non-invasive treatment to increase muscle tone in the tissues of the throat and thereby reduce snoring. The duration of snoring of 20 chronic snorers was recorded by voice-activated tape recorder for 7 nights both before and after treatment. The therapeutic intervention consisted of instruction in singing technique and singing exercises which subjects were directed to practice for 20 minutes a day for 3 months. Compliance was encouraged by a further visit and regular telephone follow-ups. Snoring was on average reduced, especially in subjects who performed the exercises accurately and consistently and who were not overweight. Those who did best, in addition, had no nasal problems and began snoring only in middle age. It was concluded that a further randomised controlled study focusing on this group would appear justified and is being planned.

\subsection{Stress/anxiety (2 clinical trials/2 systematic reviews)}

\subsubsection{Flower remedies}

The aim of a double-blind, placebo-controlled randomised clinical trial ${ }^{235}$ was to investigate the efficacy of 'Five Flower Remedy ${ }^{\circledR}$ also known as Rescue Remedy ${ }^{\circledR}$, for examination anxiety in healthy university students. One hundred university students were recruited. They were registered at the University of Exeter, aged between 18 to 65 years, and enrolled to undertake university examinations between May to July 1998. Participants were randomised to take one to four doses of either 'Five Flower Remedy' or indistinguishable placebo during day 1 to 7 of the experiment. Self- 
reported anxiety was quantified using the 40-item Spielberger State-Trait Anxiety Inventory (STAI) as the primary outcome measure. Visual analogue scales (VAS) were used as the secondary outcome measure to allow the determination of daily anxiety scores. $45 \%$ of volunteers completed the study (21 subjects in the experimental and 24 in the placebo group). Reporting of anxiety, as measured by the state component of the STAI at enrolment, was not found to differ between verum and placebo and no gender differences were noted. However, self-reports of trait anxiety were found to be lower in men compared to women at enrolment. Eight days before examination, men reported significantly lower levels of state anxiety but no significant differences were subsequently identified the evening before the examination. No significant differences were identified between or within groups for mean values of the daily VAS scores or indeed the linear contrast variable constructed to determine any trends associated with the weekly VAS reports. It was concluded that 'Five Flower Remedy' had no specific effects in treating anxiety under these trial conditions.

\subsubsection{Herbal medicine}

A systematic review and meta-analysis ${ }^{236}$ was aimed at assessing the evidence for or against the efficacy of kava extract as a symptomatic treatment for anxiety. Six independent literature searches were performed. Experts on the subject were contacted to provide further information. There were no restrictions regarding the language of publication. Double-blind, randomised, placebo-controlled trials of oral kava extract for the treatment of anxiety were included. All publications were blinded before assessment by a person not involved in the study. Data were extracted in a standardised, predefined fashion independently by the two reviewers. The methodological quality of all trials was assessed. Superiority of kava extract over placebo was suggested by all seven reviewed trials. The meta-analysis of three trials suggests a significant difference in the reduction of the total score on the Hamilton Rating Scale for Anxiety in favour of kava extract (weighted mean difference, 9.69; 95\% confidence interval, 3.54-15.83). These data therefore imply that kava extract is superior to placebo as a symptomatic treatment for anxiety.

\subsubsection{Autogenic training}

The aim of a systematic review ${ }^{237}$ was to evaluate all controlled trials of autogenic training (AT) as a means of reducing stress and anxiety levels in human subjects. A search for all published and unpublished controlled trials was carried out in four major databases. Eight such trials were located, all of which we included. The majority of trials were methodologically flawed. A range of outcome measures was used, with Spielberger's State-Trait Anxiety Inventory being the most popular. Deviations from the accepted technique of AT were conspicuous and trials using classical AT were in the minority. Seven trials reported positive effects of AT in reducing stress. One study showed no such benefit. Since one trial had used AT in combination with another technique, visual imagery, no conclusion can be drawn about the effect of AT in this case. Our conclusion therefore is that no firm conclusions could be drawn from this systematic review. AT, properly applied, remains to be tested in controlled trials that are appropriately planned and executed.

\subsubsection{Floatation therapy}

The objective of a pilot study ${ }^{238}$ was to conduct a preliminary investigation of the effects of floatation spa therapy on quality of life in patients with osteoarthritis to see if controlled trials are warranted. Fourteen patients with chronic osteoarthritis of the weight-bearing joints were included in this uncontrolled trial. The intervention consisted of six weekly sessions of floatation spa therapy. SF36, AIMS2 and MYMOP quality-of-life questionnaires were used as outcome measures. All patients improved. Differences between baseline and discharge scores showed statistically 
significant improvement for MYMOP, but not AIMS2 or SF36. Controlled trials of floatation spa therapy for patients with osteoarthritis are therefore warranted.

\subsection{Stroke (1 clinical trial, 6 systematic reviews)}

\subsubsection{Acupuncture}

Several recent reports claim that acupuncture may be a useful addition to conventional stroke rehabilitation. The aim of this systematic review ${ }^{239}$ was to critically review these data. All controlled trials published on this subject were identified by systematic literature searches. Without exception, these trials suggest positive effects of acupuncture on functional recovery. None of them, however, attempted to account for a possible placebo effect. Several other methodological flaws must be considered as well. It is therefore concluded that, according to the data published to date, the evidence that acupuncture is a useful adjunct for stroke rehabilitation is encouraging but not compelling.

As more data have emerged since the publication of this article, we felt it necessary to update it with a further systematic review. ${ }^{240}$ Four independent computerised literature searches (in MEDLINE, Cochrane Controlled Trials register, EMBASE, and CISCOM databases) were conducted in June 1999. All randomised controlled trials that compared any form of needle insertion acupuncture to any form of non-acupuncture control intervention in the treatment of human stroke patients were included. Data were independently extracted by two authors and arbitrated by a third. The methodological quality of the included studies was assessed using the Jadad score. Nine randomised controlled trials with a total sample size of 538 patients were included. Two studies were assessor blind, one was subject blind, and one was assessor and subject blind. Two studies exclusively used manual acupuncture, five only electro-acupuncture, and two used both. Outcome measures used were Scandinavian Stroke Scale, Chinese Stroke Scale or Recovery Scale, Barthel index, Nottingham Health Profile, motor function, balance and days in hospital. Of the nine studies, six yielded a positive result suggesting that acupuncture is effective, and three produced a negative finding implying that acupuncture is not superior to control treatment. Only two studies obtained a Jadad score of more than 3. These methodologically best trials showed no significant effect of acupuncture. It was concluded that, based on the evidence of rigorous randomised controlled trials, there is no compelling evidence to show that acupuncture is effective in stroke rehabilitation. Further, better-designed studies are warranted.

Any adjunctive therapy that may reduce persistent disability after stroke should be considered. Acupuncture is used for this purpose, but there is conflicting evidence on its effectiveness. Patients with a recent $(<4$ weeks) episode of stroke were randomized to receive 12 sessions of either real or sham acupuncture during 2 weeks. ${ }^{241}$ The primary outcome was the change in Barthel activities of daily living score at the end of treatment. Secondary outcome measures included National Institutes of Health Stroke Scale score, motoricity index, and quality of life (EQ-5D [EuroQoL-5

Dimensional form] and EQ-VAS [EuroQoL- Visual Analog Scale]). Assessments were carried out by blinded physicians. A total of 116 patients (56 in the real acupuncture group and 60 in the sham group) were randomized, and 98 (real, 48; sham, 50) completed treatment and the 2-week assessment. Patient blinding by means of the sham acupuncture device was successful. Acupuncture was well tolerated except for 1 seizure during a real acupuncture session. The improvements in the Barthel scores were 4 points (interquartile range [IQR], 0-8) vs 3 points (IQR, 0-7) in the real and sham acupuncture groups, respectively $(P=.38)$. The secondary outcome measures also essentially showed no significant effect of acupuncture. Post hoc analysis by baseline severity showed a greater improvement in leg function in the subgroup with baseline Barthel score less than the median 
(median score, 6): 22 points (IQR, 0-37) vs 4 points (IQR, 0-4) in the acupuncture and sham control groups, respectively $(P=.02)$. We concluded that acupuncture is not superior to sham treatment for recovery in activities of daily living and health-related quality of life after stroke, although there may be a limited effect on leg function in more severely affected patients.

Acupuncture is frequently advocated as an adjunct treatment during stroke rehabilitation. The aim of this review ${ }^{242}$ was to assess its effectiveness in this setting. We searched 25 databases and 12 major Korean traditional medicine journals from their inception to October 2009. We included randomized controlled trials, with no language restrictions, that compared the effects of acupuncture (with or without electrical stimulation) with sham acupuncture. We assessed the methodologic quality of the trials using the Cochrane risk-of-bias criteria and the PEDro (Physiotherapy Evidence Database) scale. Ten of 664 potentially relevant studies met our inclusion criteria. For acute and subacute stages after stroke, we included seven trials. A meta-analysis of the five studies that assessed functionality did not show a significant difference in favour of acupuncture, with high heterogeneity. A post-hoc sensitivity analysis of three trials with low risk of bias did not show beneficial effects of acupuncture on activities of daily living at the end of the intervention period ( $\mathrm{n}$ $=244$; standard mean difference $0.07,95 \%$ confidence interval $[\mathrm{CI}]-0.18$ to $0.32 ; \mathrm{I}(2)=0 \%$ ) or after follow-up ( $\mathrm{n}=244$; standard mean difference $0.10,95 \% \mathrm{CI}-0.15$ to 0.35 ; $\mathrm{I}(2)=0 \%)$. For the chronic stage after stroke, three trials tested effects of acupuncture on function according to the Modified Ashworth Scale; all failed to show favourable effects. Our meta-analyses of data from rigorous randomized sham-controlled trials did not show a positive effect of acupuncture as a treatment for functional recovery after stroke

Systematic reviews of acupuncture for stroke rehabilitation have been contradictory. This overview $^{243}$ is an attempt to summarize and critically evaluate this evidence. Our comprehensive search strategy located 17 systematic reviews covering different aspects of stroke rehabilitation. Six reviews drew positive conclusions. However, important caveats apply, and the most reliable reviews were negative. Therefore it is concluded that acupuncture is not an evidence-based treatment during stroke rehabilitation.

\subsubsection{Cupping}

Cupping is often used for stroke rehabilitation in Asian countries. Currently, no systematic review of this topic is available. The aim of this systematic review ${ }^{244}$ is to summarize and critically evaluate the evidence for and against the effectiveness of cupping for stroke rehabilitation. Thirteen databases were searched from their inception through March of 2010 without language restrictions. Prospective clinical trials were included if cupping was tested as the sole treatment or as an adjunct to other conventional treatments for stroke rehabilitation. We found 43 potentially relevant articles, of which 5 studies including 3 randomized clinical trials (RCTs) and 2 uncontrolled observational studies (UOSs) met our inclusion criteria. Cupping was compared with acupuncture, electroacupuncture and warm needling. Some superior effects of cupping were found in two of the RCTs when compared to acupuncture in hemiplegic shoulder pain and high upper-limb myodynamia after stroke. The other RCT failed to show favorable effects of cupping when compared to acupuncture and warm needling in patients with hemiplegic hand edema. The two UOSs reported favorable effects of cupping on aphasia and intractable hiccup after stroke. There are not enough trials to provide evidence for the effectiveness of cupping for stroke rehabilitation because most of the included trials compared the effects with unproven evidence and were not informative. Future RCTs seem warranted but must overcome the methodological shortcomings of the existing evidence 


\subsubsection{Moxibustion}

Positive effects of moxibustion for stroke rehabilitation may be seen in real clinical practice. Currently, no systematic reviews are available. The aim of this systematic review ${ }^{245}$ was to analyze the trial data on the effectiveness of moxibustion for stroke rehabilitation. Fourteen databases were searched without language restriction. Randomized clinical trials were included if moxibustion was tested as the sole treatment or as an adjunct to other treatments for stroke rehabilitation. Nine randomized clinical trials met our inclusion criteria. Three randomized clinical trials reported favorable effects of moxibustion plus standard care on motor function versus standard care alone $(\mathrm{N}=142$; standardized mean difference $=0.72 ; 95 \%$ confidence interval, 0.37 to $1.08 ; \mathrm{P}<0.0001)$. Three randomized clinical trials compared the effects of moxibustion on activities of daily living alone but failed to show favorable effects of moxibustion. In conclusion, this systematic review found limited effectiveness of moxibustion as an adjunct to standard care in stroke rehabilitation

\subsection{Syndrome X (1 clinical trial)}

The objectives of this study ${ }^{76}$ were to explore autogenic training (AT) as a treatment for psychological morbidity, symptomology, and physiological markers of stress among women with chest pain, a positive exercise test for myocardial ischemia, and normal coronary arteries (cardiac syndrome X). Fifty-three women with cardiac syndrome X (mean T SD age, $57.1 \mathrm{~T} 8$ years) were randomized to an 8-week AT program or symptom diary control. Symptom severity and frequency, Hospital Anxiety and Depression Scale, Spielberger State-Trait Anxiety Inventory, Cardiac Anxiety Questionnaire (CAQ), and Ferrans and Powers Quality of Life Index (QLI), blood pressure, heart rate, electrocardiogram, and plasma catecholamines were measured before and after intervention and at the 8-week follow-up. Women who underwent AT had improved symptom frequency $(8.04 \mathrm{~T}$ 10.08 vs $1.66 \mathrm{~T} 2.19, \mathrm{P}$ G 0.001$)$ compared with control women and reduced symtom severity $(2.08$ $\mathrm{T} 1.03$ vs $1.23 \mathrm{~T} 1.36, \mathrm{P}=0.02)$ and frequency $(6.11 \mathrm{~T} 3.17 \mathrm{vs} 1.66 \mathrm{~T} 2.19, \mathrm{P} \mathrm{G} 0.001)$ post-AT compared with baseline within group. Within-group improvements among women who underwent AT include QLI health functioning (17.80 T 5.74 vs $19.41 \mathrm{~T} 5.19, \mathrm{P}=0.04)$ and CAQ fear $(1.53 \mathrm{~T}$ $0.61 \mathrm{vs} 1.35 \mathrm{~T} 0.56, \mathrm{P}=0.02)$ post-AT and QLI health functioning (17.80 T 5.74 vs $20.09 \mathrm{~T} 5.47, \mathrm{P}$ $=0.01)$, CAQ fear (1.53 T 0.61 vs $1.30 \mathrm{~T} 0.67, \mathrm{P}=0.002)$, CAQ total $(1.42 \mathrm{~T} 0.54 \mathrm{vs} 1.29 \mathrm{~T} 0.475$, $\mathrm{P}=0.04)$, Spielberger State-Trait Anxiety Inventory trait anxiety (42.95 T 11.19 vs $38.68 \mathrm{~T} 11.47, \mathrm{P}$ $=0.01)$, and QLI quality of life (20.67 T 5.37 vs $21.9 \mathrm{~T} 4.89, \mathrm{P}=0.02)$ at follow-up. We concluded that an 8-week AT program improves symptom frequency, with near-significant improvements in symptom severity in women with cardiac syndrome X.

\subsection{Temporomandibular joint dysfunction (1 systematic review)}

The objective of this systematic review ${ }^{246}$ was to summarise the data from randomised clinical trials of acupuncture for temporomandibular joint dysfunction. Four independent computerised literature searches were performed. Only randomised trials were admitted in which acupuncture was tested versus sham acupuncture, standard therapy, or no treatment at all. Data were extracted in a predefined, standardised fashion. Six reports met the inclusion and exclusion criteria, representing 3 distinct trials. Overall, their results suggest that acupuncture might be an effective therapy for temporomandibular joint dysfunction. However, none of the studies was designed to control for a placebo effect. Even though all studies are in accordance with the notion that acupuncture is effective for temporomandibular joint dysfunction, this hypothesis requires confirmation through more rigorous investigations. 


\subsection{Tinnitus (2 systematic reviews)}

\subsubsection{Acupuncture}

Tinnitus is a prevalent condition for which patients may seek treatment with acupuncture since no conventional treatment has been shown to be effective. Our objective therefore was to summarise and critically review all randomised clinical trials on the efficacy of acupuncture as a treatment for tinnitus. ${ }^{247}$ Four independent computerised literature searches (MEDLINE, Cochrane Controlled Trials Register, EMBASE, and CISCOM) were conducted in December 1998 using the keywords acupuncture and tinnitus. All randomised clinical trials that compared any form of acupuncture with any control intervention in the treatment of tinnitus were included. Data were extracted by 2 authors independently. The methodological quality of the included randomised clinical trials was assessed using the Jadad score. Six randomised clinical trials were included in the review, 4 of which used crossover design. Four studies used manual acupuncture and 2 used electroacupuncture. Five of six studies used inconsistent acupoints. Three studies scored 3 points or more on the Jadad scale. Main outcome measurements were visual analogue scale scores for loudness, annoyance, and awareness of tinnitus; subjective severity scale scores for tinnitus; or Nottingham Health Profile scores. Two non-blinded studies showed a positive result, whereas 4 blinded studies showed no significant effect of acupuncture. Acupuncture, it was concluded, has therefore not been demonstrated to be efficacious as a treatment for tinnitus on the evidence of rigorous randomised clinical trials.

\subsubsection{Herbal medicine}

A further systematic review ${ }^{248}$ was performed to summarise all randomised clinical trials of Ginkgo biloba for tinnitus. Five such studies were identified. The results suggest that extracts of Ginkgo biloba are effective in treating tinnitus. Only one study produced a negative result and that may have been related to the dose being suboptimal ( 2 x $14.6 \mathrm{mg}$ extract per day). All other studies used much higher doses (120 mg - $160 \mathrm{mg}$ extract per day). The average methodological quality of these studies was, however, poor.

\subsection{Ulcerative colitis (1 systematic review)}

Complementary and alternative medicine (CAM) is increasingly used for treatment of inflammatory bowel disease (IBD). Acupuncture-type treatments are among the most popular options. Several studies have reported that moxibustion is effective in ulcerative colitis (UC). The objective of this review $^{249}$ was to assess the clinical evidence for or against moxibustion as a treatment for UC. We searched the literature using 18 databases from their inception to February 10, 2010, without language restrictions. We included randomized clinical trials (RCTs), in which human patients with UC were treated with moxibustion. Studies were included if they were placebo-controlled or controlled against a drug therapy or no treatment group. The methodological quality of all RCTs was assessed using the Cochrane risk of bias. In total, five RCTs were included. All were of low methodological quality. They compared the effects of moxibustion with conventional drug therapy. Three tested moxibustion against sulfasalazine and two against sulfasalazine plus other drugs. A meta-analysis of five RCTs showed favorable effects of moxibustion on the response rate compared to conventional drug therapy $(\mathrm{n}=407$; risk ratio $=1.24,95 \% \mathrm{CI}=1.11$ to $1.38 ; \mathrm{P}<0.0001$; heterogeneity: $\mathrm{I} 2=16 \%$ ). In conclusion, current evidence is insufficient to show that moxibustion is an effective treatment of UC. Most of included trials had high risk of bias. More rigorous studies seem warranted 


\subsection{Varicose veins (1 systematic review)}

The objective of this systematic review ${ }^{250}$ was to assess the evidence for or against horse-chestnut seed extract (HCSE) as a symptomatic treatment of chronic venous insufficiency (CVI). Five computerised literature searches were performed. There were no restrictions on the language of publication. Double-blind, randomised clinical trials of oral HCSE for patients with CVI were included. Identifiers were removed from all publications before assessment. Data were extracted in a standardised, pre-defined manner. Trial outcomes and the methodological quality of each trial were independently assessed by the 2 reviewers. The superiority of HCSE is suggested by all placebo-controlled studies. The use of HCSE is associated with a decrease of the lower-leg volume and a reduction in leg circumference at the calf and ankle. Symptoms such as leg pain, pruritus, and a feeling of fatigue and tenseness are reduced. Five comparative trials against the reference medication indicate that HCSE and $O$-( $\beta$-hydroxyethyl)-rutosides are equally effective. One trial suggests a therapeutic equivalence of HCSE and compression therapy. Adverse effects are usually mild and infrequent. These data therefore imply that HCSE is superior to placebo and as effective as reference medications in alleviating the objective signs and subjective symptoms of CVI. Thus, HCSE represents a treatment option for CVI that is worth considering.

This review has recently been updated and published on the Cochrane Database. ${ }^{251}$

\subsection{Weight reduction (2 clinical trials/7 systematic reviews)}

\subsubsection{Acupuncture}

Acupuncture and acupressure are often advocated and used as a means of controlling appetite and reducing body weight, supported by case-series and uncontrolled studies that show encouraging results. This systematic review ${ }^{252}$ evaluated placebo/sham-controlled clinical trials published on this topic. Two independent literature searches identified four such studies. None of these is without significant methodological flaws and their results are contradictory. The two relatively rigorous trials show no effect on body weight. Therefore, no clear picture emerges to show that acupuncture/ acupressure is effective in reducing appetite or body weight. Claims that these forms of treatment have specific effects on these conditions are therefore not based on well-performed clinical trials.

The purpose of this study ${ }^{253}$ was to compare the effects of diet and exercise vs acupuncture, diet and exercise on the body weight and related parameters of adult women. Twenty-seven obese women with a body fat percentage of more than $30 \%$ were randomized into three groups. The first experimental group had diet and exercise, whereas the second experimental group had diet, exercise and acupuncture. The control group received no intervention at all. The study period lasted for 8 weeks. Body weight, skin fold thickness, body mass index and fat mass were measured before and after 8 weeks. Body mass index and fat mass, decreased significantly (Po0.05) in both experimental groups when compared with the control group. However, there was no significant difference between the two experimental groups. Changes in lean body mass after 8 weeks were not significantly different from those in the control group. It is concluded that acupuncture combined with diet and exercise does not generate larger reductions in body weight, fat mass or body mass index than diet and exercise alone.

\subsubsection{Any type of CAM}

The prevalence of obesity is increasing at an alarming rate and a plethora of complementary therapies are on offer claiming effectiveness for reducing body weight. The aim of this systematic 
review ${ }^{254}$ is to critically assess the evidence from randomized controlled trials (RCTs) and systematic reviews of complementary therapies for reducing body weight. Literature searches were conducted on Medline, Embase, Amed, and the Cochrane Library until January 2004. Handsearches of relevant medical journals and bibliographies of identified articles were conducted. There were no restrictions regarding the language of publication. Trial selection, quality assessment and data abstraction were performed systematically and independently by two authors. Data from RCTs and systematic reviews, which based their findings on the results of RCTs, were included. Six systematic reviews and 25 additional RCTs met our inclusion criteria and were reviewed. The evidence related to acupuncture, acupressure, dietary supplements, homeopathy and hypnotherapy. Except for hypnotherapy, Ephedra sinica and other ephedrine-containing dietary supplements the weight of the evidence is not convincing enough to suggest effectiveness. For these interventions, small effects compared with placebo were identified. In conclusion, our findings suggest that for most complementary therapies, the weight of the evidence for reducing body is not convincing. Hypnotherapy, E. sinica and other ephedrine-containing dietary supplements may lead to small reductions in body weight. However, the intake of $E$. sinica and ephedrine is associated with an increased risk of adverse events. Interventions suggesting positive effects in single RCTs require independent replication.

\subsubsection{Food supplements}

An overview of systematic reviews included 9 such articles. ${ }^{255}$ No clear evidence emerged for any food supplement as a viable option for weight reduction.

\subsubsection{Chitosan}

Chitosan is promoted in the US and other countries as an oral remedy to reduce fat absorption and has now been incorporated as a major constituent into several over-the-counter remedies. The primary aim of this study is to investigate the clinical effectiveness of oral chitosan for body weight reduction. ${ }^{256}$ Thirty-four overweight volunteers were included in a randomised placebo-controlled double-blind trial. Subjects were assigned to receive either four capsules of chitosan or indistinguishable placebo twice daily for 28 consecutive days. Measurements were taken at baseline, after 14 and 28 days of treatment. Subjects maintained their normal diet and documented the type and amount of food consumed. Adverse events were assessed and compliance monitored. Data from 30 subjects were entered into an intention-to-treat analysis. After four weeks of treatment, body mass index, serum cholesterol, triglycerides, vitamin $\mathrm{A}, \mathrm{D}, \mathrm{E}$ and $\beta$-carotene were not significantly different in subjects receiving chitosan compared to those receiving placebo. Vitamin $\mathrm{K}$ was significantly increased after four weeks in the chitosan group compared with placebo $(\mathrm{P}<0.05)$. Compliance was $91.5 \%$ and $96.0 \%$ for chitosan and placebo groups respectively. The above data therefore suggest that chitosan in the administered dosage, without dietary alterations, does not reduce body weight in overweight subjects. No serious adverse events were reported.

A systematic review and meta-analysis of chitosan summarised the published evidence for or against the effectiveness for the treatment of obesity. ${ }^{257}$ A comprehensive search strategy was designed to identify all randomised, placebo-controlled, double-blind trials on the subject. Five trials met all inclusion criteria. All studies originate from Italy and were published in the same Italian journal, over a 2-year time period. These studies administered chitosan or placebo in addition to a hypocaloric diet and suggest that, in such circumstances, the medication with chitosan results in a significantly greater weight loss compared with placebo. The meta-analysis implied that the mean difference in terms of weight reduction between chitosan and placebo is $3.28 \mathrm{~kg}$. However, several concerns were raised and the results have to be viewed with caution. It was therefore concluded that 
the clinical effectiveness of chitosan as a means of weight reduction needs to be confirmed by independent rigorous trials.

\subsubsection{Chromium}

The aim of this meta-analysis was to assess the evidence of chromium picolinate for reducing body weight. ${ }^{258}$ Literature searches were conducted on MEDLINE, EMBASE, The Cochrane Library, AMED and CISCOM. Nine experts and four manufacturers of commercial preparations containing chromium picolinate were asked to contribute published and unpublished studies. There were no restrictions regarding the language of publication. The screening of studies, selection, data extraction, validation and the assessment of methodological quality were performed independently by two reviewers. To be included, studies were required to state that they were randomised, doubleblind and placebo-controlled, and report on body weight. Ten trials met all inclusion criteria and provided data which were suitable for statistical pooling. For body weight a significant differential effect was found in favour of chromium picolinate (weighted mean difference: $-1.1 \mathrm{~kg} ; 95 \%$ confidence interval $(\mathrm{CI}):-1.8$ to $-0.4 \mathrm{~kg}, n=489$ ). Sensitivity analysis suggests that this effect is largely dependent on the results of a single trial (weighted mean difference: $-0.9 \mathrm{~kg} ; 95 \% \mathrm{CI}:-2.0$ to $0.2 \mathrm{~kg}, n=335$ ). Three of the reviewed trials reported on adverse events, indicating their absence in the treatment groups. In conclusion, our meta-analysis suggests a relatively small effect of chromium picolinate compared with placebo for reducing body weight. The clinical relevance of the effect is debatable and the lack of robustness means that the result has to be interpreted with caution.

\subsubsection{Garcinia}

The aim of this systematic review ${ }^{259}$ is to examine the efficacy of Garcinia extract, hydroxycitric acid (HCA) as a weight reduction agent, using data from randomised clinical trials (RCTs). Electronic and nonelectronic searches were conducted to identify relevant articles, with no restrictions in language or time. Two independent reviewers extracted the data and assessed the methodological quality of included studies. Twenty-three eligible trials were identified and twelve were included. Nine trials provided data suitable for statistical pooling. The meta-analysis revealed a small, statistically significant difference in weight loss favouring HCA over placebo (MD: - 0.88 $\mathrm{kg} ; 95 \%$ CI: $-1.75,-0.00)$. Gastrointestinal adverse events were twice as common in the HCA group compared with placebo in one included study. It is concluded that the RCTs suggest that Garcinia extracts/HCA can cause short-term weight loss. The magnitude of the effect is small, and the clinical relevance is uncertain. Future trials should be more rigorous and better reported

\subsubsection{Guar gum}

The purpose of this meta-analysis was to determine the efficacy of the dietary fibre guar gum as a therapeutic option for reducing body weight by conducting a meta-analysis of randomised controlled trials. ${ }^{260}$ Literature searches were performed on the electronic databases MEDLINE, EMBASE, BIOSIS, AMED, and the Cochrane Library. Manufacturers of commercial guar gum preparations and experts on the subject were contacted to provide any published or unpublished trials. For inclusion, trials had to state that they were randomised, double-blinded and placebo-controlled, used guar gum monopreparations, and reported body weight as an endpoint. No language restrictions were imposed. Two reviewers independently extracted data in a standardised manner according to pre-defined criteria and evaluated methodological quality using the scoring system developed by Jadad. Discrepancies were settled through discussion. Thirty-four trials were identified and 20 could be included. Eleven trials provided data that were suitable for statistical pooling. The meta-analysis indicated a non-significant difference in patients receiving guar gum compared with patients 
receiving placebo (mean weighted difference $-0.04 \mathrm{~kg}$; $95 \%$ confidence interval (CI): -2.2 to 2.1 ). Analysis of six trials with similar methodological features corroborates these findings (mean weighted difference $-0.3 \mathrm{~kg} ; 95 \% \mathrm{CI}:-4.0$ to 3.5$)$. Adverse events most frequently reported were abdominal pain, flatulence, diarrhoea, and cramps. Overall, 11 patients $(3 \%)$ dropped out owing to adverse events. It was concluded that this meta-analysis suggests that guar gum is not efficacious for reducing body weight. Considering the adverse events associated with its use, the risks of taking guar gum outweigh its benefits for this indication. Therefore, guar gum cannot be recommended as a treatment for lowering body weight.

\section{Effectiveness/effects/validity of CM modalities (unrelated to specific conditions)}

\subsection{Diagnostic methods}

\subsubsection{Any technique}

The prevalence of complementary medicine in most industrialised countries is impressive and increasing. Discussions of the topic often focus on therapeutic approaches and neglect diagnostic methods specific for complementary medicine. This article ${ }^{261}$ summarises the data available on such "alternative" diagnostics. Scientific evaluations of these are scant, and most techniques have never been properly validated. The ones that have, can be demonstrated to be not reproducible, sensitive, or specific. The ones that have not, should be regarded as such until shown otherwise by rigorous testing. Therefore it seems that "alternative" diagnostic methods may seriously threaten the safety and health of patients submitted to them. Orthodox doctors should be aware of the problem and inform their patients accordingly. An update of this review found more evidence but arrived at essentially the same conclusion. ${ }^{262}$

\subsubsection{Iridology}

Iridologists claim to be able to diagnose medical conditions through abnormalities of pigmentation in the iris. This technique is popular in many countries. Therefore it is relevant to ask whether it is valid. The aim of this article ${ }^{263}$ was to systematically review all interpretable tests of the validity of iridology as a diagnostic tool. Three independent literature searches were performed to identify all blinded tests. Data were extracted in a pre-defined, standardised fashion. Four case control studies were found. The majority of these investigations suggest that iridology is not a valid diagnostic method. The validity of iridology as a diagnostic tool is not supported by scientific evaluations. Patients and therapists should be discouraged from using this method.

\subsection{3 $\underline{\text { Reflexology }}$}

The aim of this study was to investigate whether manual techniques used by reflexologists can be used as a valid method of diagnosis. ${ }^{264}$ Three experienced reflexologists took part in this study. Eighteen adults with one or more of six specified conditions were identified from primary care records. Two reflexologists, who were blinded to the patients' conditions and monitored, then examined each patient's feet and rated the probability that each of the six conditions was present. There is little evidence that the distribution of ratings varies with the status of the condition. Receiver operating curves suggest that this diagnostic method is very poor at distinguishing between 
the presence and absence of conditions. Inter-rater reliability (kappa) scores were very low, providing no evidence of agreement between the examiners. It was therefore concluded that despite certain limitations to the data provided by this study, the results do not suggest that reflexology techniques are a valid method of diagnosis.

\subsection{Therapeutic methods}

\subsection{1 $\quad$ Acupressure}

An overview of systematic review found 9 such articles on acupressure. ${ }^{265}$ Most were of such low quality and suggested positive effects on a range of conditions. We concluded that the effectiveness of acupressure has not been demonstrated for any condition.

\subsubsection{Acupuncture general}

The aim of this systematic review ${ }^{266}$ was to summarize randomized clinical trials (RCTs) assessing the effectiveness of acupuncture as published in Korean literature. Systematic searches were conducted on eight Korean medical databases. Manual searches were also conducted through eight major Korean medical journals. The methodological quality was assessed using a Jadad score. Studies evaluating needle acupuncture or auricular acupuncture (AA) with or without electrical stimulation were considered if they were sham or placebo-controlled or controlled against a comparative intervention. We also excluded acupuncture as an adjuvant to other treatments and other forms of acupuncture were excluded. Seven hundred and nine possibly relevant studies were identified and 10 RCTs were included. The methodological quality of the trials was generally poor. Manual acupuncture was compared to placebo acupuncture in four studies of patients with chronic low back pain, shoulder pain, premenstrual syndrome and allergic rhinitis. Three studies tested AA (two trials) and electroacupuncture (one trial) against no treatment, while three trials compared acupuncture with other active therapeutic controls. The methodological limitations of the included trials make their contribution to the current clinical evidence of acupuncture somewhat limited. The trial for premenstrual syndrome, shoulder pain and chronic low back pain added a limited contribution among those included RCTs. However, well-designed RCTs of acupuncture with a rigorous methodology are in progress or have been completed in Korea and will contribute to establish or contribute to the current progress of research in this field.

Many trials of acupuncture and numerous systematic reviews have recently become available. Their conclusions are far from uniform. In an attempt to find the most reliable type of evidence, this $\operatorname{article}^{267}$ provides an overview of Cochrane reviews of acupuncture. Such reviews were studied, their details extracted, and they were categorized as: reviews with a negative conclusion (no evidence that acupuncture is effective); reviews that were inconclusive; and reviews with a positive or tentatively positive conclusion. Thirty-two reviews were found, covering a wide range of conditions. Twenty-five of them failed to demonstrate the effectiveness of acupuncture. Five reviews arrived at positive or tentatively positive conclusions and two were inconclusive. The conditions that are most solidly backed up by evidence are chemotherapy-induced nausea/vomiting, postoperative nausea/vomiting, and idiopathic headache. It is concluded that Cochrane reviews of acupuncture do not suggest that this treatment is effective for a wide range of conditions.

The aim of this systematic review ${ }^{266}$ was to summarize randomized clinical trials (RCTs) assessing the effectiveness of acupuncture as published in Korean literature. Systematic searches were conducted on eight Korean medical databases. Manual searches were also conducted through eight major Korean medical journals. The methodological quality was assessed using a Jadad score. 
Studies evaluating needle acupuncture or auricular acupuncture (AA) with or without electrical stimulation were considered if they were sham or placebo-controlled or controlled against a comparative intervention. We also excluded acupuncture as an adjuvant to other treatments and other forms of acupuncture were excluded. Seven hundred and nine possibly relevant studies were identified and 10 RCTs were included. The methodological quality of the trials was generally poor. Manual acupuncture was compared to placebo acupuncture in four studies of patients with chronic low back pain, shoulder pain, premenstrual syndrome and allergic rhinitis. Three studies tested AA (two trials) and electroacupuncture (one trial) against no treatment, while three trials compared acupuncture with other active therapeutic controls. The methodological limitations of the included trials make their contribution to the current clinical evidence of acupuncture somewhat limited. The trial for premenstrual syndrome, shoulder pain and chronic low back pain added a limited contribution among those included RCTs. However, well-designed RCTs of acupuncture with a rigorous methodology are in progress or have been completed in Korea and will contribute to establish or contribute to the current progress of research in this field.

The aim of this article ${ }^{268}$ is to evaluate trends over time in the development of the evidence-base of acupuncture. A comparison of two series of systematic reviews was conducted. The first related to the evidence-base in 2000, the second related to 2005. Both employed virtually the same methodology and criteria for evaluation. The results indicate that the evidence base has increased for 13 of the 26 conditions included in this comparison. For 7 indications it has become more positive (i.e. favoring acupuncture) and for 6 it had changed in the opposite direction. It is concluded, that acupuncture research is active. The emerging clinical evidence seems to imply that acupuncture is effective for some but not all conditions.

\subsubsection{Acupuncture in veterinary medicine}

Acupuncture is a popular complementary treatment option in human medicine. Increasingly, owners also seek acupuncture for their animals. The aim of the systematic review reported here ${ }^{269}$ was to summarize and assess the clinical evidence for or against the effectiveness of acupuncture in veterinary medicine. Systematic searches were conducted on Medline, Embase, Amed, Cinahl, Japana Centra Revuo Medicina and Chikusan Bunken Kensaku. Hand-searches included conference proceedings, bibliographies, and contact with experts and veterinary acupuncture associations.

There were no restrictions regarding the language of publication. All controlled clinical trials testing acupuncture in any condition of domestic animals were included. Studies using laboratory animals were excluded. Titles and abstracts of identified articles were read, and hard copies were obtained. Inclusion and exclusion of studies, data extraction, and validation were performed independently by two reviewers. Methodologic quality was evaluated by means of the Jadad score. Fourteen randomized controlled trials and 17 nonrandomized controlled trials met our criteria and were, therefore, included. The methodologic quality of these trials was variable but, on average, was low. For cutaneous pain and diarrhea, encouraging evidence exists that warrants further investigation in rigorous trials. Single studies reported some positive intergroup differences for spinal cord injury, Cushing's syndrome, lung function, hepatitis, and rumen acidosis. These trials require independent replication. On the basis of the findings of this systematic review, there is no compelling evidence to recommend or reject acupuncture for any condition in domestic animals. Some encouraging data do exist that warrant further investigation in independent rigorous trials.

\subsubsection{Acupuncture in human medicine}

Even though widely used in today's clinical practice, acupuncture has remained a controversial subject. Many reviews are currently available but most lack a critical stance and some are overtly promotional. The aim of this overview ${ }^{270}$ is to provide a balanced, critical analysis of the existing 
evidence. Some of the original concepts of traditional acupuncture are not supported by good scientific evidence. Several plausible theories attempt to explain how acupuncture works but none are proved beyond doubt. The clinical effectiveness of acupuncture continues to attract controversy. Many controlled clinical trials and numerous systematic reviews of these studies have been published. Considerable problems are encountered when interpreting these data. Heterogeneity is a significant drawback of both clinical trials and systematic reviews. Some of the controversies may be resolved through the use of the new 'placebo needles' which enable researchers to adequately control for placebo effects of acupuncture. The majority of studies using such devices fails to show effects beyond a placebo response. Acupuncture has been associated with serious adverse events but most large-scale studies suggest that these are probably rare. Nonserious adverse effects occur in 7$11 \%$ of all patients. In conclusion, acupuncture remains steeped in controversy. Some findings are encouraging but others suggest that its clinical effects mainly depend on a placebo response.

\subsubsection{Alexander technique}

Alexander technique (AT), a process of psychophysical re-education, is being promoted for a range of medical conditions. This systematic review ${ }^{271}$ was aimed at critically evaluating the evidence for or against the effectiveness of AT. Computerised literature searches were performed in five databases to locate all controlled clinical trials of AT in any human condition. Data were extracted independently by two reviewers according to pre-defined criteria. The information was summarised in tabular and narrative form. The methodological quality of the primary studies was assessed using the Jadad score. Four clinical trials met our inclusion exclusion criteria. Only two of these trials were methodologically sound and clinically relevant. Their results were promising and implied that AT is effective in reducing the disability of patients suffering from Parkinson's disease and in improving pain behaviour and disability in patients with back pain.

\subsubsection{Aloe vera}

Aloe vera is a popular herbal remedy in the UK. It is marketed for a range of conditions. This systematic review ${ }^{272}$ was aimed at defining its clinical effectiveness. Four independent literature searches were conducted in MEDLINE, EMBASE, BIOSIS and Cochrane Library. Only controlled clinical trials (on any indication) were included. There were no restrictions on the language of publication. All trials were read by both authors and data were extracted in a standardised, predefined manner. Ten studies were located. They suggest that oral administration of aloe vera might be a useful adjunct for lowering blood glucose in diabetic patients as well as for reducing blood lipid levels in patients with hyperlipidaemia. Topical application of aloe vera is not an effective preventative for radiation-induced injuries. It might be effective for genital herpes and psoriasis. Whether it promotes wound healing is unclear. There are major caveats associated with all these statements. Even though there are some promising results, clinical effectiveness of oral or topical aloe vera is not sufficiently defined at present.

\subsubsection{Anthroposophical medicine}

Anthroposophical medicine (AM) was developed by Steiner and Wegman and wants to be seen as an adjunct to medicine. This article ${ }^{273}$ represents the attempt of a critical analysis of AM. It demonstrates the attempt of a critical analysis of AM. It shows that AM is based on irrational concepts. The effectiveness of either the anthroposophic medicines or the entire system is unproven. Contrary to numerous assertions, AM, is not risk-free. A risk-benefit evaluation therefore fails to be positive. 


\subsubsection{Aromatherapy}

Aromatherapy is becoming increasingly popular; however there are few clear indications for its use. Our aim was to systematically review the literature on aromatherapy in order to discover whether any clinical indication may be recommended for its use. ${ }^{274}$ Five computerised literature searches were performed to retrieve all randomised clinical trials of aromatherapy. The methodological quality of the trials was assessed using the Jadad score. All trials were evaluated independently by both authors and data were extracted in a pre-defined, standardised fashion. Twelve trials were located: six of them had no independent replication; six related to the relaxing effects of aromatherapy combined with massage. These studies suggest that aromatherapy massage has a mild, transient anxiolytic effect. Based on a critical assessment of the six studies relating to relaxation, the effects of aromatherapy are probably not strong enough for it to be considered for the treatment of anxiety. The hypothesis that it is effective for any other indication is therefore not supported by the findings of rigorous clinical trials.

\subsection{9 $\underline{\text { Ayurveda }}$}

The aim of this article ${ }^{275}$ was to provide an overview of systematic reviews evaluating the effectiveness of Ayurvedic treatments. We searched five electronic databases and our departmental files without restrictions of time or language. All systematic reviews and their methodological quality were evaluated independently by both authors. Results: Of 34 potentially relevant publications, five met our inclusion criteria. All systematic reviews related to Ayurvedic herbal medicines. All but one were of good methodological quality. The subject areas were hyperlipidemia, schizophrenia, rheumatoid arthritis, diabetes and anti-inflammatory effects. One review arrived at clearly negative, three at equivocal and one at positive overall conclusions. We concluded that due to several important caveats, our evaluation fails to provide compelling evidence that Ayurvedic treatments are effective for any conditions.

\subsubsection{Bach flower remedies}

Bach flower remedies continue to be popular and its proponents make a range of medicinal claims for them. The aim of this systematic review ${ }^{276}$ was to critically evaluate the evidence for these claims. Five electronic databases were searched without restrictions on time or language. All randomised clinical trials of flower remedies were included. Seven such studies were located. All but one were placebo-controlled. All placebo-controlled trials failed to demonstrate efficacy. It is concluded that the most reliable clinical trials do not show any differences between flower remedies and placebos

\subsubsection{Biofeedback}

The aim of this systematic review ${ }^{277}$ was to provide a general overview of the evidence on the therapeutic effectiveness of biofeedback. Literature searches were carried out to locate all systematic reviews on this subject. These were evaluated according to predefined criteria. Twenty reviews met the inclusion criteria. Their results suggest that various forms of biofeedback are effective as adjunctive treatments of anismus, faecal incontinence, paediatric migraine, rheumatoid arthritis, stroke rehabilitation and temporomandibular disorders. The evidence is inconclusive for asthma, chronic pain, erectile dysfunction, gastrointestinal disorders, hypertension, insomnia, obstructive pulmonary disease, stress management, and tinnitus. The evidence is clearly negative for atopic eczema, back pain, tension-type headache and cervicogenic headache. It was concluded that biofeedback is an effective therapy for a range of conditions and that clinicians should consider using it more frequently. 


\subsubsection{Chiropractic}

Chiropractic was defined by D.D. Palmer as "a science of healing without drugs". About 60,000 chiropractors currently practice in North America, and, worldwide, billions are spent each year for their services. This article ${ }^{278}$ attempts to critically evaluate chiropractic. The specific topics include the history of chiropractic; the internal conflicts within the profession; the concepts of chiropractic, particularly those of subluxation and spinal manipulation; chiropractic practice and research; and the efficacy, safety, and cost of chiropractic. A narrative review of selected articles from the published chiropractic literature was performed. For the assessment of efficacy, safety, and cost, the evaluation relied on previously published systematic reviews. Chiropractic is rooted in mystical concepts. This led to an internal conflict within the chiropractic profession, which continues today. Currently, there are two types of chiropractors: those religiously adhering to the gospel of its founding fathers and those open to change. The core concepts of chiropractic, subluxation, and spinal manipulation, are not based on sound science. Back and neck pain are the domains of chiropractic but many chiropractors treat conditions other than musculoskeletal problems. With the possible exception of back pain, chiropractic spinal manipulation has not been shown to be effective for any medical condition. Manipulation is associated with frequent mild adverse effects and with serious complications of unknown incidence. Its cost-effectiveness has not been demonstrated beyond reasonable doubt. The concepts of chiropractic are not based on solid science and its therapeutic value has not been demonstrated beyond reasonable doubt.

The "Activator": is an instrument used by many chiropractors for spinal manipulation. The aim of this systematic review ${ }^{279}$ is to critically evaluate all randomised clinical trials of this therapeutic approach. Six electronic databases were searched to identify all relevant studies. They were validated according to predefined criteria and their methodology quality was assessed by two independent reviewers. Six trials met the inclusion criteria. Two studies reach related to neck pain and back pain while two related to hypertension and phobias. Their methodological quality was poor (average Jadad score $=1.5$ of 5 possible points). The results were ambiguous for neck pain and back pain and suggested positive effects for hypertension and phobias. Due to the paucity and poor quality of the primary data, it is concluded that he effectiveness of spinal manipulation with the "Activator" is not supported by good evidence.

Most chiropractors advise patients to have regular maintenance treatments with spinal manipulation, even in the absence of any symptoms or diseases. This article ${ }^{280}$ evaluates the evidence for or against this approach. No compelling evidence was found to indicate that chiropractic maintenance therapy effectively prevents symptoms or diseases. As spinal manipulation has repeatedly been associated with considerable harm, the risk benefit balance of chiropractic maintenance care is not demonstrably positive. Therefore there are no good reasons to recommend it.

The aim of this systematic review ${ }^{281}$ was to promote an independent and critical evaluation of 11 randomised clinical trials (RCTs) of chiropractic funded by the National Centre for Complementary and Alternative Medicine (NCCAM). Electronic searches were conducted to identify all relevant RCTs. Key data were extracted and the risk of bias of each study was determined. Ten RCTs were included, mostly related to chiropractic spinal manipulation for musculoskeletal problems. Their quality was frequently questionable. Several RCTs failed to report adverse effects and the majority was not described in sufficient detail to allow replication. The criticism repeatedly aimed at NCCAM seems justified, as far as their RCTs of chiropractic is concerned. It seems questionable whether such research is worthwhile 


\subsubsection{Colonic Irrigation}

A review ${ }^{282}$ disclosed numerous therapeutic claims for this treatment. None of them was supported by good evidence.

\subsubsection{Distant healing}

The aim of this article ${ }^{283}$ was to conduct a systematic review of the available data on the efficacy of any form of 'distant healing' (prayer, mental healing, therapeutic touch, or spiritual healing) as treatment for any medical condition. Studies were identified by five electronic searches and by contact with researchers in the field. Studies with the following features were included: random assignment, placebo or other adequate control, publication in peer-reviewed journals, clinical investigations, and use of human participants. Two investigators independently extracted data on study design, sample size, type of intervention, type of control, direction of effect (supporting or refuting the hypothesis), and nature of the outcomes. A total of 23 trials involving 2774 patients met the inclusion criteria and were analysed. Heterogeneity of the studies precluded a formal metaanalysis. Of the trials, 5 examined prayer as the distant healing intervention, 11 assessed noncontact therapeutic touch, and 7 examined other forms of distant healing. Of the 23 studies, 13 (57\%) yielded statistically significant treatment effects, 9 showed no effect over control interventions, and 1 showed a negative effect. The methodological limitations of several studies make it difficult to draw definitive conclusions about the efficacy of distant healing. However, given that approximately $57 \%$ of trials showed a positive treatment effect, the evidence thus far suggests that healing merits further study.

Because new trial data have since become available, this evidence was recently updated. ${ }^{284}$ Eight non-randomised and 9 randomised clinical trials were located. The majority of the rigorous trials do not to support the hypothesis that distant healing has specific therapeutic effects. The results of two studies furthermore suggest that distant healing can be associated with adverse effects. Since the publication of our previous systematic review in 2000, several rigorous new studies have emerged. Collectively they shift the weight of the evidence against the notion that distant healing is more than a placebo.

Distant healing, a treatment that is transmitted by a healer to a patient at another location, is widely used, although good scientific evidence of its efficacy is sparse. This trial was aimed at assessing the efficacy of one form of distant healing on common skin warts as a simple clinical model. ${ }^{285} \mathrm{~A}$ total of 84 patients with warts were randomly assigned either to a group that received 6 weeks of distant healing by one of 10 experienced healers or to a control group that received a similar preliminary assessment but no distant healing. The primary outcomes were the number of warts and their mean size at the end of the treatment period. Secondary outcomes were the change in Hospital Anxiety and Depression Scale and the patients' subjective experiences. Both the patients and the evaluator were blinded to group assessment. The baseline characteristics of the patients were similar in the distant healing $(n=41)$ and control groups $(n=43)$. The mean number and size of warts per person did not change significantly during the study. The number of warts increased by 0.2 in the healing group and decreased by 1.1 in the control group (difference [healing to control] $=-1.3 ; 95 \%$ confidence interval $=-1.0$ to $3.6, P=0.25$ ). Six patients in the distant healing group and 8 in the control group reported a subjective improvement $(P=0.63)$. There were no significant betweengroup differences in the depression and anxiety scores. Distant healing from experienced healers had no effect on the number or size of patients' warts. 


\subsubsection{Feldenkrais Method}

The Feldenkrais Method (FM) is being promoted for a range of medical conditions. This systematic review $^{286}$ is aimed at summarising and critically evaluating the results of randomised controlled trials of FM. Literature searches were carried out in 7 electronic databases. All randomised controlled trials of FM were included regardless of indication. No language restrictions were applied. The data extracted and valuated by two independent reviewers. The methodological quality of the primary studies was assessed with the Jadad score. Six studies met our inclusion criteria. They were all burdened with significant methodological weaknesses. The indications included multiple sclerosis, neck/shoulder problems and chronic back pain. All but one trial re[ported positive results. We concluded that evidence of the FM is encouraging but, due to the paucity and low quality of studies, by no means compelling.

\subsubsection{Flower remedies}

Flower remedies (also called 'Bach' flower remedies) are used by an increasing number of individuals, and many health claims have been made for them. No systematic review of flower remedies has so far been published. The aim of this systematic review is to summarise and critically analyse the data from all available controlled clinical trials of flower remedies. ${ }^{287}$ Six databases were searched to identify all controlled clinical trials of flower remedies in humans for any medical condition. No language restrictions were applied. Key data were validated and extracted into table format according to pre-defined criteria. Statistical pooling was not possible, and results were evaluated in narrative form. Four studies met the inclusion/exclusion criteria. Two trials suggested a positive outcome. Those studies that controlled for placebo-effects and minimised selection bias through randomisation failed to demonstrate effects beyond a placebo response. The hypothesis that flower remedies are associated with effects beyond a placebo response is not supported by data from rigorous clinical trials.

\subsubsection{Frankincense}

The objective of this systematic review ${ }^{288}$ was to assess evidence from randomised clinical trials about the effectiveness of extracts of Boswellia serrata (frankincense). Electronic searches on Medline, Embase, Cinahl, Amed, and Cochrane Library. Hand searches of conference proceedings, bibliographies, and departmental files. All randomised clinical trials of $B$ serrata extract as a treatment for any human medical condition were included and studies of $B$ serrata preparations combined with other ingredients were excluded. Titles and abstracts of all retrieved articles were read and hard copies of all relevant articles were obtained. Selection of studies, data extraction and validation were done by the author. The Jadad score was used to evaluate the methodological quality of all included trials. Of 47 potentially relevant studies, seven met all inclusion criteria (five placebo controlled, two with active controls). The included trials related to asthma, rheumatoid arthritis, Crohn's disease, osteoarthritis, and collagenous colitis. Results of all trials indicated that $B$ serrata extracts were clinically effective. Three studies were of good methodological quality. No serious safety issues were noted. It was concluded that the evidence for the effectiveness of $B$ serrata extracts is encouraging but not compelling.

\subsubsection{Cimicifuga racemosa}

Cimicifuga racemosa is widely employed to relieve menopausal symptoms for its hormonal-like action. However, recent experimental studies have not found an estrogenic action by this plant. The purpose of this systematic review ${ }^{289}$ was to analyse all experimental studies (in vivo and in vitro) performed on C. racemosa to elucidate its mechanism of action. Animal in vitro experiments on $C$. 
racemosa were identified through computerised literature searches performed on Medline (PubMed), Embase, Amed, CISCOM and Cochrane Library databases. In addition, bibliographies of the articles thus located were scanned for further relevant publications and manufacturers of commercial C. racemosa preparations were asked to contribute published and unpublished material. No language restrictions were imposed. A total of 15 animal and 15 in vitro studies on C. racemosa have been found. Their results suggest that $C$. racemosa possesses a central activity instead of a hormonal effect. Further biological and chemical investigations are required to define its mechanism of action and to identify the compounds responsible for its actions.

\subsubsection{Garlic}

The objective of this review ${ }^{290}$ is to update and assess the clinical evidence based on rigorous trials of the effectiveness of garlic (A. sativum). Systematic searches were carried out in Medline, Embase, Amed, the Cochrane Database of Systematic Reviews, Natural Standard, and the Natural Medicines Comprehensive Database (search date December 2006). Our own files, the bibliographies of relevant papers and the contents pages of all issues of the review journal FACTwere searched for further studies. No language restrictions were imposed. To be included, trials were required to state that they were randomized and double blind. Systematic reviews and meta-analyses of garlic were included if based on the results of randomized, double-blind trials. The literature searches identified six relevant systematic reviews and meta-analysis and double-blind randomized trials (RCT) that were published subsequently. These relate to cancer, common cold, hypercholesterolemia, hypertension, peripheral arterial disease and pre-eclampsia. The evidence based on rigorous clinical trials of garlic is not convincing. For hypercholesterolemia, the reported effects are small and may therefore not be of clinical relevance. For reducing blood pressure, few studies are available and the reported effects are too small to be clinically meaningful. For all other conditions not enough data are available for clinical recommendations.

\subsubsection{Ginger}

Zingiberis rhizoma is used as a broadspectrum antiemetic. We, therefore, conducted a comprehensive review ${ }^{291}$ of the literature to summarize the pharmacological and clinical effects of this popular plant material. Although clinical and experimental studies suggest that ginger has some antiemetic properties, clinical evidence beyond doubt is only available for pregnancy-related nausea and vomiting. Meta-analyses could not demonstrate the postoperative antiemetic effectiveness, and effect in motion sickness or nausea/vomiting of other ethiology. It also remains to be confirmed that proprietary ginger preparations are clinically useful to alleviate osteoarthritic or other pain, although there is no doubt that ginger constituents interfere with the inflammatory cascade and the vanilloid nociceptor. Ginger exerts in vitro antioxidative, antitumorigenic and immunomodulatory effects and is an effective antimicrobial and antiviral agent. Animal studies demonstrate effects on the gastrointestinal tract, the cardiovascular system, on experimental pain and fever, antioxidative, antilipidemic and antitumor effects, as well as central and other effects. The most relevant human pharmacological studies require a confirmatory study to exclude interaction of ginger preparations with platelet aggregation. Pharmacokinetic data are only available for [6]-gingerol and zingiberene. Preclinical safety data do not rule out potential toxicity, which should be monitored especially following ginger consumption over longer periods.

\subsubsection{Ginseng}

Ginseng is one of the most popular herbal remedies, and a number of health claims are made for it. This systematic review ${ }^{292}$ provides an evaluation of the current evidence for or against the efficacy of ginseng root extract. Searches of the computerised literature databases MEDLINE, EMBASE, 
BIOSIS, CISCOM and the Cochrane Library were performed to retrieve double-blind, randomised, placebo-controlled trials of ginseng root extract for any indication. Manufacturers and experts were contacted to provide additional information. There were no restrictions regarding the language of publication. The outcome and methodological quality of all trials were independently assessed by two reviewers. Sixteen trials met the inclusion criteria and were reviewed. These trials related to physical performance, psychomotor performance and cognitive function, immunomodulation, diabetes mellitus and herpes simplex type-II infections. The evidence found for ginseng root extract is compelling for none of these indications. Based on these data, it is concluded that the efficacy of ginseng root extract is not established beyond reasonable doubt for any of these indications. The widespread use of ginseng as a herbal remedy warrants more rigorous investigations to assess its efficacy and safety.

This overview ${ }^{293}$ is focused on Panax ginseng, one of the most commonly used herbal remedies. The currently available data regarding its clinical effi cacy is somewhat confusing, despite the fact that many clinical trials and systematic reviews have been published. The risks of Panax ginseng seem to be only minor. Numerous mechanisms of action have been described. Future studies of this fascinating herbal remedy seem warranted but they should overcome the methodological limitations of the previous research.

\subsubsection{Herbal medicine}

Because use of herbal remedies is increasing, a risk-benefit profile of commonly used herbs is needed. A review ${ }^{294}$ provided a clinically oriented overview of the efficacy and safety of ginkgo, St John's wort, ginseng, echinacea, saw palmetto, and kava. Wherever possible, assessments were based on systematic reviews of randomised clinical trials. Encouraging data support the efficacy of some of these popular herbal medicinal products, and the potential for doing good seems greater than that for doing harm. The published evidence suggests that ginkgo is of questionable use for memory loss and tinnitus but has some effect on dementia and intermittent claudication. St John's wort is efficacious for mild to moderate depression, but serious concerns exist about its interactions with several conventional drugs. Well-conducted clinical trials do not support the efficacy of ginseng to treat any condition. Echinacea may be helpful in the treatment or prevention of upper respiratory tract infections, but trial data are not fully convincing. Saw palmetto has been shown in short-term trials to be efficacious in reducing symptoms of benign prostatic hyperplasia. Kava is an efficacious short-term treatment for anxiety. None of these herbal medicines is free from adverse effects. Because the evidence is incomplete, risk-benefit assessments are not completely reliable, and much knowledge is still lacking.

Our aim was to summarise and critically evaluate the evidence from randomised clinical trials for the effectiveness of individualised herbal medicine in any indication. ${ }^{295}$ Searches were carried out of electronic databases and approaches were made to experts in the field to identify randomised, controlled clinical trials of individualised herbal medicine in any indication. Independent data extraction and assessment of methodological quality were performed by two authors and best evidence synthesis. Three randomised clinical trials of individualised herbal medicine were identified. Statistically nonsignificant trends favouring active over placebo treatment in osteoarthritis of the knee probably result from large baseline differences and regression to the mean. Individualised treatment was superior to placebo in four of five outcome measures in the treatment of irritable bowel syndrome, but was inferior to standardised herbal treatment in all outcomes. Individualised herbal treatment was no better than placebo in the prevention of chemotherapyinduced toxicity. We concluded that there is a sparsity of evidence regarding the effectiveness of individualised herbal medicine and no convincing evidence to support the use of individualised herbal medicine in any indication. 


\subsubsection{Homoeopathy (7 systematic reviews)}

An important meta-analysis by Linde et al has suggested that the clinical effects of homoeopathic remedies cannot be fully explained by placebo effects. Thus it is relevant to ask whether such remedies are equally effective as conventional drug treatments irrespective of indication. Various literature searches located six comparative trials. ${ }^{296}$ All were burdened with serious methodological flaws. The results of these trials are non-uniform. A clear trend is not visible. It is concluded that at present the relative efficacy of homoeopathic remedies is not known.

The homoeopathic remedy most frequently studied in placebo-controlled clinical trials (regardless of indication) is Arnica montana. Our aim was to systematically review the clinical efficacy of homoeopathic arnica. ${ }^{297}$ Four computerised literature searches were performed to retrieve all placebo-controlled studies on the subject. Data were extracted in a pre-defined, standardised fashion independently by both authors. There were no restrictions on the language of publication. Eight trials fulfilled all inclusion criteria. Most related to conditions associated with tissue trauma. Most of these studies were burdened with severe methodological flaws. On balance, they do not suggest that homoeopathic arnica is more efficacious than placebo. The claim that homoeopathic arnica is efficacious beyond a placebo effect is not supported by rigorous clinical trials.

The meta-analysis by Linde et al of trials of homoeopathic remedies concluded that such medications have clinical effects beyond placebo. As this raises important principal issues, a reanalysis of the same data was performed. ${ }^{298}$ Only trials of the highest methodological standard were included. Studies on material dilutions or remedies not following the 'like cures like' principle of homoeopathy were excluded. Five studies fulfilled the inclusion criteria. The results suggest that homoeopathic remedies are associated with the same clinical effects as placebo.

A further article was an attempt to clarify its effectiveness based on recent systematic reviews. ${ }^{299}$ Electronic databases were searched for systematic reviews/meta-analyses on the subject. Seventeen articles fulfilled the inclusion/exclusion criteria. Six of them related to re-analyses of one landmark meta-analysis. Collectively they implied that the overall positive result of this meta-analysis is not supported by a critical analysis of the data. Eleven independent systematic reviews were located. Collectively they failed to provide strong evidence in favour of homoeopathy. In particular, there was no condition which responds convincingly better to homoeopathic treatment than to placebo or other control interventions. Similarly, there was no homoeopathic remedy that was demonstrated to yield clinical effects that are convincingly different from placebo. It was concluded that the best clinical evidence for homoeopathy available to date does not warrant positive recommendations for its use in clinical practice.

This systematic review ${ }^{300}$ evaluated the evidence of any type of therapeutic or preventive intervention testing homeopathy for childhood and adolescence ailments. Systematic literature searches were conducted through January 2006 in MEDLINE, EMBASE, AMED, CINAHL, Cochrane Central, British Homeopathic Library, ClinicalTrials.gov, and the UK National Research Register. Bibliographies were checked for further relevant publications. Studies were selected according to predefined inclusion and exclusion criteria. All double-blind, placebo-controlled randomized clinical trials of any homeopathic intervention for preventing or treating childhood and adolescence ailments were included. According to the classification of the World Health Organization, the age range defined for inclusion was 0 to 19 years. Study selection, data extraction, and assessment of methodological quality were performed independently by 2 reviewers. A total of 326 articles were identified, 91 of which were retrieved for detailed evaluation. Sixteen trials that assessed 9 different conditions were included in the study. With the exception of attention- 
deficit/hyperactivity disorder and acute childhood diarrhea (each tested in 3 trials), no condition was assessed in more than 2 double-blind randomized clinical trials. The evidence for attentiondeficit/hyperactivity disorder and acute childhood diarrhea is mixed, showing both positive and negative results for their respective main outcome measures. For adenoid vegetation, asthma, and upper respiratory tract infection each, 2 trials are available that suggest no difference compared with placebo. For 4 conditions, only single trials are available. The evidence from rigorous clinical trials of any type of therapeutic or preventive intervention testing homeopathy for childhood and adolescence ailments is not convincing enough for recommendations in any condition.

The objective of this review ${ }^{301}$ was to evaluate the evidence for and against the effectiveness of homeopathy. The Cochrane Database of Systematic Reviews (generally considered to be the most reliable source of evidence) was searched in January 2010. Cochrane reviews with the term "homeopathy" in the title, abstract or keywords were considered. Protocols of reviews were excluded. Six articles met the inclusion criteria. Each of the six reviews was examined for specific subject matter; number of clinical trials reviewed; total number of patients involved; and authors' conclusions. The reviews covered the following conditions: cancer, attention-deficit hyperactivity disorder, asthma, dementia, influenza and induction of labour. The findings of the reviews were discussed narratively (the reviews' clinical and statistical heterogeneity precluded meta-analysis). In conclusion, the findings of currently available Cochrane reviews of studies of homeopathy do not show that homeopathic medicines have effects beyond placebo

In September 2006, the UK licensing authorities have agreed that, within EU regulation, homeopathic medicines will be allowed to make claims in the absence of proof of effectiveness. Other member states have taken or will take similar action. Yet the effectiveness of homeopathy remains a controversial issue. The aim of this article ${ }^{302}$ is to define how the evidence-base for or against the effectiveness of homeopathic remedies has changed over time and discuss possible emerging trends. For this purpose, a comparison of two series of systematic reviews related to 18 conditions was conducted. The first described the evidence-base in 2000, the second in 2005. Both employed the same methodology and criteria for evaluation. The results indicate that the evidence base has changed a little. The data that did emerge failed to alter he overall evidence in a positive sense and, in several areas, changed it in a negative direction. We concluded that homeopathy is not based on solid evidence and, over time, this evidence seems to get more negative.

\subsubsection{Homotoxicology}

Homotoxicology is a form of therapy that uses homoeopathically diluted remedies with a view of eliminating toxins from the body. Numerous clinical studies have claimed efficacy. The aim of this systematic review ${ }^{303}$ was to summarise and critically evaluate the evidence from rigorous clinical trials of this form of therapy. Seven electronic databases were searched for all studies of homotoxicological medicines for any human condition. To be included, trials had to be randomised and placebo-controlled. Data from such studies were validated and extracted according to predefined criteria. Their methodological quality was formally assessed using the Jadad score. Key data of all included trials were tabulated and summarised in narrative form. Seven trials met our inclusion criteria. Their Jadad scores indicated mostly a high methodological standard. The trials tested the efficacy of seven different medicines for seven different indications. The results were positive in all but one study. Important flaws were found in all trials. These render the results of the primary studies less reliable than their high Jadad scores might suggest. We concluded that, despite mostly positive findings and high ratings on the Jadad score, the placebo-controlled, randomised clinical trials of homotoxicology fail to demonstrate the efficacy of this therapeutic approach. 


\subsubsection{Kombucha}

Kombucha has become a popular complementary remedy. The aim of this systematic review was to critically evaluate the evidence related to its efficacy and safety. ${ }^{304}$ Computerised literature searches were carried out to locate all human medical investigations of kombucha regardless of study design. Data were extracted and validated by the current author and are reported in narrative form. No clinical studies were found relating to the efficacy of this remedy. Several case reports and case series raise doubts about the safety of kombucha. They include suspected liver damage, metabolic acidosis and cutaneous anthrax infections. One fatality is on record. On the basis of these data it was concluded that the largely undetermined benefits do not outweigh the documented risks of kombucha. It can therefore not be recommended for therapeutic use.

\subsubsection{Massage therapy}

Massage therapies belong to the oldest treatments of mankind. They are popular in many countries, yet no recent analysis of the clinical effectiveness has been published. Our aim was to provide a critical review of the world literature related to the clinical effectiveness of classic massage therapy. ${ }^{305}$ A computerised literature search $(1970$ - 1993) was performed, for which the MEDLINE database system was used. Additional references were also found by other means, i.e. searching our personal files and the reference lists of articles and books. Our initial intention to include only controlled trials had to be abandoned because of the extreme rarity of such reports. The lack of scientific rigour of the studies retrieved was the most outstanding finding of the search. Thus only very few scientifically proven indications for massage emerged through this review, and even these were less well established than today's accepted standards require.

The aim of this review ${ }^{306}$ is to evaluate trends in the development of the evidence-base for the effectiveness of massage therapy. For this purpose, a comparison of two systematic reviews was conducted. The first related to the evidence-base in 2000, the second to 2005. Both employed the same methodology and criteria for evaluation. The results indicate that, in several areas, the evidence has become more solid and, for anxiety and back pain, it has become more positive. For a host of other indications, the evidence seems encouraging, but more studies are required to test the effectiveness of massage therapy as well as its use for specific conditions.

\subsubsection{Mind body therapies}

The aim of this article ${ }^{307}$ is to evaluate trends in the development of the evidence base for autogenic training, hypnotherapy, and relaxation therapy. For this purpose, a comparison of 2 series of systematic reviews was conducted. The first is related to the evidence base in 2000, the second to that in 2005. Both employed virtually the same methodology and criteria for evaluation. The results of our comparisons show considerable changes during the observation period. The weight of the evidence has become stronger for several indications, and the direction of the evidence has been altered in a positive sense in several conditions. Applying the rules of evidence-based medicine, the following mind-body therapies are now supported by strong evidence: hypnotherapy for labor pain and relaxation therapy for anxiety and insomnia, as well as for nausea and vomiting induced by chemotherapy. It is concluded that an evidence-based approach for mind-body therapies is constructive and can generate positive results.

\subsubsection{Moxibustion}

Several systematic reviews (SRs) have assessed the effectiveness of moxibustion for a range of conditions, often with contradictory conclusions. ${ }^{308}$ Our aim was to provide a critical evaluation and 
summary of these data. Electronic searches were conducted to locate all SRs of moxibustion for any condition. Data were extracted by two authors according to predefined criteria. Ten SRs met our inclusion criteria, which related to the following conditions: cancer, ulcerative colitis, stroke rehabilitation, constipation, hypertension, pain conditions and breech presentation. Their conclusions were contradictory in several instances. Relatively clear evidence emerged to suggest that moxibustion is effective for breech presentation. Based on evidence from the currently available SRs, the effectiveness of moxibustion has been demonstrated for several conditions; however, due to the poor quality of the primary studies, there remains considerable uncertainty

\subsubsection{Neural therapy}

In German-speaking countries, local injection of anaesthetic is a popular form of treatment, either as 'neural therapy', a complementary method intended to stimulate adaptive body functions, or as pure analgesia. This review ${ }^{309}$ was aimed at summarising the published evidence for or against neural therapy. Its results suggested that neither the rationale nor the individual mechanisms, nor its therapeutic efficacy have been investigated scientifically. Recent data show that the analgesic effect does not depend on the injection of local anaesthetic. It is conceivable that this form of treatment acts via a mechanism known as diffuse noxious inhibition control.

\subsubsection{Reflexology}

Reflexology has become one of the most frequently used treatment modalities within complementary medicine. This level of popularity makes it desirable for healthcare professionals to understand the essential facts about it. The aim of this article ${ }^{310}$ was to review the existing literature on reflexology and compile the facts. Particular emphasis is put on clinical trials. Two independent, computerised literature searches were performed. All articles with factual information were included. Various other sources of information were used as well. Particular emphasis was on data relating to prevalence, effectiveness, safety and costs. For reviewing the effectiveness of reflexology, only controlled clinical trials were considered for a systematic review. The prevalence of reflexology is variable. Only few controlled clinical trials on the subject have been published. Collectively they do not support the notion that reflexology is associated with specific therapeutic effects. The data on safety and costs are similarly insufficient. Reflexology is popular, yet little factual information exists about it. On the basis of the existing data, it seems possible, even probable that its perceived benefit is brought about by non-specific effects.

The objective of this systematic review ${ }^{311}$ was to evaluate the evidence for and against the effectiveness of reflexology for treating any medical condition. Six electronic databases were searched from their inception to February 2009 to identify all relevant randomised controlled trials (RCTs). No language restrictions were applied. RCTs of reflexology delivered by trained reflexologists to patients with specific medical conditions were included. Condition studied, study design and controls, primary outcome measures, follow-up, and main results were extracted. 18 RCTs met all the inclusion criteria. The studies examined a range of conditions: anovulation, asthma, back pain, dementia, diabetes, cancer, foot oedema in pregnancy, headache, irritable bowel syndrome, menopause, multiple sclerosis, the postoperative state and premenstrual syndrome. There were $>1$ studies for asthma, the postoperative state, cancer palliation and multiple sclerosis. Five RCTs yielded positive results. Methodological quality was evaluated using the Jadad scale. The methodological quality was often poor, and sample sizes were generally low. Most higher-quality trials did not generate positive findings. It was concluded that the best evidence available to date does not demonstrate convincingly that reflexology is an effective treatment for any medical condition. 
The aim of this update ${ }^{312}$ is to critically evaluate the evidence for or against the effectiveness of reflexology in patients with any type of medical condition. Six electronic databases were searched to identify all relevant randomised clinical trials (RCTs). Their methodological quality was assessed independently by the two reviewers using the Jadad score. Overall, 23 studies met all inclusion criteria. They related to a wide range of medical conditions. The methodological quality of the RCTs was often poor. Nine high quality RCTs generated negative findings; and five generated positive findings. Eight RCTs suggested that reflexology is effective for the following conditions: diabetes, premenstrual syndrome, cancer patients, multiple sclerosis, symptomatic idiopathic detrusor over-activity and dementia yet important caveats remain. It is concluded that the best clinical evidence does not demonstrate convincingly reflexology to be an effective treatment for any medical condition

\subsubsection{Reiki}

The aim of this systematic review ${ }^{313}$ is to summarise and critically evaluate the evidence for the effectiveness of reiki. We searched the literature using 23 databases from their respective inceptions through to November 2007 (search again 23 January 2008) without language restrictions. Methodological quality was assessed using the Jadad score. The searches identified 205 potentially relevant studies. Nine randomised clinical trials (RCTs) met our inclusion criteria. Two RCTs suggested beneficial effects of reiki compared with sham control on depression, while one RCT did not report intergroup differences. For pain and anxiety, one RCT showed intergroup differences compared with sham control. For stress and hopelessness a further RCT reported effects of reiki and distant reiki compared with distant sham control. For functional recovery after ischaemic stroke there were no intergroup differences compared with sham. There was also no difference for anxiety between groups of pregnant women undergoing amniocentesis. For diabetic neuropathy there were no effects of reiki on pain. A further RCT failed to show the effects of reiki for anxiety and depression in women undergoing breast biopsy compared with conventional care. Discussion: In total, the trial data for any one condition are scarce and independent replications are not available for each condition. Most trials suffered from methodological flaws such as small sample size, inadequate study design and poor reporting. In conclusion, the evidence is insufficient to suggest that reiki is an effective treatment for any condition. Therefore the value of reiki remains unproven.

\subsubsection{Rhodiola rosea}

The objective of this systematic review ${ }^{314}$ was to critically assess the current evidence from randomized clinical trials (RCTs) for or against the effectiveness or efficacy of Rhodiola rosea. Systematic literature searches were performed in six electronic databases: AMED (1985-July 2009), CINAHL (1982-July 2009), The Cochrane Library (search in July 2009), EMBASE (1974-July 2009), MEDLINE (1950-July 2009) and Web of Science (searched in July 2009). No language restrictions were imposed. Reference lists of all retrieved articles were searched, and experts and manufacturers were contacted for unpublished RCT. RCTs testing the efficacy or effectiveness of mono-preparations of $\mathrm{R}$. rosea as sole treatment administered orally against a control intervention in any human individual suffering from any condition or healthy human volunteers were included. Studies were selected, data extracted, and quality assessed by two independent reviewers. Eleven RCTs met the inclusion criteria; all were placebo-controlled. Six trials investigated the effects of R. rosea on physical performance, four on mental performance, and two in patients diagnosed with mental health condition. The methodological quality of most trials was moderate or good. Only few mild adverse events were reported. In conclusion, R. rosea may have beneficial effects on physical performance, mental performance, and certain mental health conditions. There is, however, a lack of independent replications of the single different studies. Five of the 10 RCTs reached more than three points on the Jadad score (i.e., good quality). More research seems warranted 


\subsubsection{Spinal manipulation}

To investigate the question whether or not spinal manipulation (for any indication) is associated with specific treatments, a systematic review of the published literature was performed. ${ }^{315}$ Literature searches were carried out in MEDLINE, EMBASE and The Cochrane Library. All sham-controlled trials of spinal manipulation were considered. Seven such studies were located. Their methodological quality was variable but three trials adhered to the highest standards of scientific rigour. Collectively these data do not show therapeutic effects beyond placebo. In particular, the three most rigorous studies were negative. The few sham-controlled trials that do exist show that this methodology is, in principle, applicable also to spinal manipulation. The results available to date suggest that the therapeutic success of spinal manipulation is largely due to a placebo effect.

The results of this systematic review were also published in full. ${ }^{316}$ It was concluded that "the most rigorous...studies suggest that spinal manipulation is not associated with clinically relevant specific therapeutic effects".

We also wanted to systematically collate and evaluate the evidence from recent systematic reviews of clinical trials of spinal manipulation. ${ }^{317}$ Literature searches were carried out in four electronic databases for all systematic reviews of the effectiveness of spinal manipulation in any indication, published between 2000 and May 2005. Reviews were defined as systematic if they included an explicit and repeatable inclusion and exclusion criteria for studies. Sixteen papers were included relating to the following conditions: back pain $(n=3)$, neck pain $(n=2)$, lower back pain and neck pain $(n=1)$, headache $(n=3)$, non-spinal pain $(n=1)$, primary and secondary dysmenorrhoea $(n=1)$, infantile colic $(n=1)$, asthma $(n=1)$, allergy $(n=1)$, cervicogenic dizziness $(n=1)$, and any medical problem $(n=1)$. The conclusions of these reviews were largely negative, except for back pain where spinal manipulation was considered superior to sham manipulation but not better than conventional treatments. Collectively these data do not demonstrate that spinal manipulation is an effective intervention for any condition. Given the possibility of adverse effects, this review does not suggest that spinal manipulation is a recommendable treatment.

\subsubsection{Spinal manipulation for the elderly}

Chiropractic spinal manipulation is popular and many elderly individuals use this therapeutic approach mostly (but not exclusively) for musculoskeletal complaints, e.g. back and neck pain. Even though numerous studies of SM have been carried out, few of them have been specifically performed with elderly patients. The evidence from rigorous clinical trials of SM for any condition is far from convincing at present. ${ }^{318}$ The data for acute back and neck pain are promising but unfortunately not fully convincing. The evidence for all other indications is even less compelling. Mild adverse effects after SM are common. Serious adverse effects are on record but their frequency is currently not known. Contra-indications for SM include osteoporosis which would seem to render SM contra-indicated for the majority of elderly individuals. Given this state of affairs, it would seem unwise to recommend SM to elderly individuals. Those who try SM despite the lack of encouraging evidence should be informed about the lack of proven benefit and about the risk which may be higher in the elderly compared to younger patients.

\subsubsection{Spinal manipulation - sources of bias}

The effectiveness of spinal manipulation as a treatment for back pain remains uncertain and controversial. This is because of methodological weakness in many of the published clinical trials and also because of markedly opposing interpretations of the primary data by different reviewers. 
We have systematically assessed a representative sample of recent reviews on this topic. ${ }^{319}$ Reviews were included in the analysis if they were published between 1993 and March 2004, were listed in PubMed with an abstract and categorised as a review or meta-analysis, and were written in English. They were also required to present the evidence from at least two referenced clinical trials of spinal manipulation for back pain and to reach a conclusion about the effectiveness of the intervention. Each review was evaluated for methodological quality. Twenty-nine reviews met the inclusion criteria. Sixteen reached an overall positive conclusion, 7 a negative conclusion and 6 a neutral conclusion regarding therapeutic effectiveness. There were statistically significant pairwise correlations between each of the three factors: direction of conclusion, methodological quality and authorship by osteopaths or chiropractors. This indicates an association between authorship by osteopaths or chiropractors and low methodological quality and positive conclusion. We conclude that the outcomes of reviews of this subject are strongly influenced by both scientific rigour and profession of authors. The effectiveness of spinal manipulation for back pain is less certain than many reviews suggest; most high quality reviews reach negative conclusions.

\subsubsection{Tai chi}

Tai chi has been claimed to generate beneficial effects with respect to a wide range of diseases. The purpose of this systematic review was to evaluate evidence from randomised clinical trials (RCTs) testing the effectiveness of tai chi for increasing aerobic capacity. Systematic searches were conducted on 14 electronic databases without restrictions on population characteristics or the language of publication. The outcome measures considered for inclusion were changes in maximal oxygen consumption as a test for aerobic capacity. Five RCTs met all inclusion criteria. Three RCTs compared the effects of tai chi with no treatment. The meta-analysis failed to show an effect of tai chi on aerobic capacity compared with sedentary controls $(n=151$, weight mean difference, $\mathrm{ml} / \mathrm{kg} / \mathrm{min}, 0.50,95 \%$ CI 21.14 to $2.15, \mathrm{p}=0.55$ ). Two RCTs compared tai chi with conventional physical exercise including brisk, low intensity and moderate intensity walking, and aerobic exercise. The results show that tai chi was not statistically significantly superior to physical exercise. In conclusion, the existing evidence does not suggest that regular tai chi is an effective way of increasing aerobic capacity.

\subsubsection{Tea tree oil}

Tea tree oil (TTO) is immensely popular for various topical applications. In vitro studies have repeatedly demonstrated that it has antibiotic activity. This study is an attempt to systematically review the evidence from randomised clinical trials for or against effectiveness of external TTO in dermatological conditions. ${ }^{320}$ Six electronic databases were searched. Methodological quantity was assessed by Jadad score. Data were extracted and validated in a standardised fashion by two independent reviewers. Only 4 trials were located. They suggest that TTO may be effective as a treatment of acne and fungal infections. The evidence is promising but by no means compelling. The adverse effects of TTO are usually mild and transient. They mainly consist of allergic reactions. It is concluded that, so far, there is no compelling evidence to show that TTO is efficacious in any dermatological condition. However, in view of promising findings, TTO deserves to be investigated more closely.

\subsubsection{Therapeutic relationship}

The therapeutic relationship is, of course, not a CAM modality but it is undoubtedly important for CAM. Throughout history, doctor-patient relationships have been acknowledged as having an important therapeutic effect, irrespective of any prescribed drug or treatment. We did a systematic review to determine whether there was any empirical evidence to support this theory. ${ }^{321} \mathrm{~A}$ 
comprehensive search strategy was developed to include 11 medical, psychological, and sociological electronic databases. The quality of eligible trials was objectively assessed by two reviewers, and the type of non-treatment care given in each trial was categorised as cognitive or emotional. Cognitive care aims to influence patients' expectations about the illness or the treatment, whereas emotional care refers to the style of the consultation (e.g. warm, empathic), and aims to reduce negative feelings such as anxiety and fear. We identified 25 eligible randomised controlled trials. 19 examined the effects of influencing patients' expectations about treatment, half of which found significant effects. None of the studies examined the effects of emotional care alone, but four trials assessed a combination of both cognitive and emotional care. Three of these studies showed that enhancing patients' expectations through positive information about the treatment or the illness, while providing support or reassurance, significantly influenced health outcomes. There is much inconsistency regarding emotional and cognitive care, although one relatively consistent finding is that physicians who adopt a warm, friendly, and reassuring manner are more effective than those who keep consultations formal and do not offer reassurance.

\subsubsection{Yoga}

Yoga has become a popular form of CAM and is often advocated for a wide range of conditions. The aim of this overview ${ }^{322}$ was to evaluate critically all systematic reviews of yoga for the symptomatic treatment of any condition. Twelve electronic databases were searched and 21 systematic reviews relating to a wide range of conditions were located. Nine systematic reviews arrived at positive conclusions, but many systematic reviews were associated with a high risk of bias. Unanimously positive evidence emerged for depression and cardiovascular risk reduction. Despite an impressive number of systematic reviews, evidence of effectiveness is positive only for two indications. 


\section{Safety of complementary therapies}

\subsection{Quality of case reports (1 systematic review)}

Case reports provide essential information on adverse effects. Yet there is little consistency in the quality and format of reporting them. In this study, ${ }^{323}$ we aimed to assess the quality of case reports of adverse effect of herbal medicinal products (HMPs) published during three time periods, 19861988, 1996-1998, and 2006-2008. We conducted literature searches in four major databases: Medline, EMBASE, AMED, and CINALH. Each case report was subject to specific inclusion criteria related to the intervention (i.e. herbal medicine) and outcome measurement (i.e. adverse effect). A 21-item scale was used to assess the quality of all included reports. Each report was categorised into low quality (score between 0 and 14), lower medium quality (score between 15 and 21), upper medium quality (score between 22 and 28), and high quality (score between 29 and 42). In total, 137 case reports were included. The percentage of high quality case reports rose from $0 \%$ in $1986-1988$ to $27.9 \%$ in $1996-1998$ and $34.2 \%$ in $2006-2008$; conversely, the percentages of low quality case reports dropped from $13.3 \%$ in 1986-1988 to $0 \%$ in 1996-1998 and 2.5\% in 2006-2008. These findings are consistent with the notion that the quality of case reports is improving. However, due to several caveats, our data should be interpreted with caution

\subsection{Toxicity of herbal medicinal products (HMPs) in general (4 systematic reviews)}

Herbal medicines have become a popular form of therapy. They are often perceived as being natural and therefore harmless. Several reviews of the recent literature on the adverse effects of herbal remedies have been conducted. ${ }^{324-327}$ Examples of allergic reactions, toxic reactions, adverse effects related to a herb's desired pharmacological actions, possible mutagenic effects, drug interactions, drug contamination, and mistaken plant identities are provided. Because of under-reporting, our present knowledge may well be just the "tip of the iceberg". Little is known about the relative safety of herbal remedies compared to synthetic drug treatments, although for some herbal remedies, the risks may be less than for conventional drugs.

\subsection{Ginkgo biloba/bleeding (1 systematic review)}

Ginkgo biloba, currently one of the most popular herbal remedies for self-medication, has repeatedly been associated with bleeding. This systematic review ${ }^{328}$ of case reports is aimed at determining whether the risk is real. Five electronic literature searches were carried out to locate all reports of bleeding associated with Ginkgo biloba mono-preparations. Twelve articles met the inclusion criteria and these were assessed by two independent reviewers according to pre-defined criteria. The likelihood of causality was also assessed. The results show that the clinical evidence for Ginkgo biloba causing bleeding is far from compelling. Pre-clinical studies yield inconsistent effects on platelet aggregation and the majority of rigorous trials refute a risk of bleeding. It is concluded that the causality between Ginkgo biloba intake and bleeding is unlikely. However, continuous vigilance seems well advised. 


\subsection{Hepatotoxicity of herbal medicinal products (1 systematic review)}

The aim of this systematic review was to evaluate the recent evidence on the association of herbal medicinal products. ${ }^{329}$ Systematic literature searches were conducted on Medline, Embase, The Cochrane Library, Amed and Ciscom. To identify additional data, searches were conducted by hand in relevant medical journals and in our own files. There were no restrictions regarding the language of publication. The screening and selection of articles and the extraction of data were performed independently by the two authors. There were no restrictions regarding the language of publication. To be included articles were required to report data on hepatotoxic events associated with the therapeutic intake of herbal medicinal products. Single medicinal herbs and combination preparations are associated with hepatotoxic events. Clinically, the spectrum ranges from transient elevations of liver enzyme levels to fulminant liver failure and death. In most instances, hepatotoxic herbal constituents are believed to be the cause while others may be due to herb-drug interactions and/or contamination. We concluded that a number of herbal medicinal products are associated with serious hepatotoxic events. The incidence of these events is largely unknown and in most cases a causal attribution is not established. The challenge for the future is to systematically research this area, educate all parties involved, and minimize patient risk.

\subsection{Herbal supplements for body weight reduction (1 systematic review)}

Herbal weight-loss supplements are marketed with claims of effectiveness. Our earlier systematic review identified data from double-blind, randomized controlled trials for a number of herbal supplements. The aim of this systematic review ${ }^{330}$ was to assess all clinical evidence of adverse events of herbal food supplements for body weight reduction for which effectiveness data from rigorous clinical trials exist. We assessed Ephedra sinica, Garcinia cambogia, Paullinia cupana, guar gum, Plantago psyllium, Ilex paraguariensis and Pausinystalia yohimbe. Literature searches were conducted on Medline, Embase, Amed and The Cochrane Library. Data were also requested from the spontaneous reporting scheme of the World Health Organization. We hand-searched relevant medical journals and our own files. There were no restrictions regarding the language of publication. The results show that adverse events including hepatic injury and death have been reported with the use of some herbal food supplements. For herbal ephedra and ephedrinecontaining food supplements an increased risk of psychiatric, autonomic or gastrointestinal adverse events and heart palpitations has been reported. In conclusion, adverse events are reported for a number of herbal food supplements, which are used for reducing body weight. Although the quality of the data does not justify definitive attribution of causality in most cases, the reported risks are sufficient to shift the risk-benefit balance against the use of most of the reviewed herbal weight-loss supplements. Exceptions are Garcinia cambogia and yerba maté, which merit further investigation.

\subsection{Cardiovascular adverse effects of HMPs (1 systematic review)}

This article summarises recent evidence regarding the serious or potentially serious cardiovascular adverse effects of herbal medicines. ${ }^{331}$ Five electronic literature databases were searched. The evidence found was mostly anecdotal. Case reports and case series indicate that life-threatening adverse effects of herbal medicines occur. Potentially serious adverse effects are arrhythmias, arteritis, cardiac glycosides overdose, chest pain, congestive heart failure, hypertension, hypotension, myocardial infarction, over-anti-coagulation, pericarditis and death. The problems relate to toxic herbal ingredients, adulteration and contamination of herbal medicinal products, and herb-drug interactions. Herbal medicines that have been implicated repeatedly include aconite, ephedra and liquorice. Because of the anecdotal nature of the evidence, it is impossible to estimate the incidence of adverse effects. In conclusion, herbal medicinal products are regularly associated 
with serious cardiovascular adverse events but the size of this problem cannot be estimated at present. Vigilance and research seem to the best way forward.

\subsection{Psychiatric \& neurological adverse effects of herbal medicines ( 1 systematic review)}

Psychiatric and neurological patients frequently try herbal medicines often under the assumption that they are safe. The aim of this systematic review ${ }^{332}$ was to provide a summary of recent data on severe psychiatric and neurological adverse effects of herbal remedies. Computerized literature searches were carried out to identify all reports of psychiatric and neurological adverse effects associated with herbal medicines. These data were subsequently extracted, validated and summarised in narrative and tabular form. Numerous case reports comprise a diverse array of adverse events including cerebral arteritis, cerebral oedema, delirium, coma, confusion, encephalopathy, hallucinations, intracerebral haemorrhage, and other types of cerebrovascular accidents, movement disorders, mood disturbances, muscle weakness, paresthesiae and seizures. Several fatalities are on record. They are due to improper use, toxicity of herbal ingredients, contamination and adulteration of preparations as well as herb/drug interactions. It was concluded that herbal medicines can cause serious psychiatric and neurological adverse effects.

\subsection{Toxicity of chaste tree (1 systematic review)}

Vitex agnus castus L. (VAC) [Verbenaceae] is a deciduous shrub that is native to Mediterranean Europe and Central Asia. Traditionally, VAC fruit extract has been used in the treatment of many female conditions, including menstrual disorders (amenorrhoea, dysmenorrhoea), premenstrual syndrome (PMS), corpus luteum insufficiency, hyperprolactinaemia, infertility, acne, menopause and disrupted lactation. The German Commission E has approved the use of VAC for irregularities of the menstrual cycle, premenstrual disturbances and mastodynia. Clinical reviews are available for the efficacy of VAC in PMS, cycle disorders, hyperprolactinaemia and mastalgia, but so far no systematic review has been published on adverse events or drug interactions associated with VAC. Therefore, this review was conducted to evaluate all the available human safety data of VAC monopreparations. Literature searches were conducted in six electronic databases, in references lists

of all identified papers and in departmental files. ${ }^{333}$ Data from spontaneous reporting schemes of the WHO and national drug safety bodies were also included. Twelve manufacturers of VACcontaining preparations and five herbalist organisations were contacted for additional information. No language restrictions were imposed. Combination preparations including VAC or homeopathic preparations of VAC were excluded. Data extraction of key data from all articles reporting adverse events or interactions was performed independently by at least two reviewers, regardless of study design. Data from clinical trials, postmarketing surveillance studies, surveys, spontaneous reporting schemes, manufacturers and herbalist organisations indicate that the adverse events following VAC treatment are mild and reversible. The most frequent adverse events are nausea, headache, gastrointestinal disturbances, menstrual disorders, acne, pruritus and erythematous rash. No drug interactions were reported. Use of VAC should be avoided during pregnancy or lactation. Theoretically, VAC might also interfere with dopaminergic antagonists. Although further rigorous studies are needed to assess the safety of VAC, the data available seem to indicate that VAC is a safe herbal medicine. 


\subsection{Toxicity of Crataegus (1 systematic review)}

Crataegus spp. (hawthorn) monopreparations are predominantly used for treating congestive heart failure. The effectiveness of hawthorn preparations (flowers with leaves; berries) is documented in a number of clinical studies, reviews and meta-analyses. The aim of this article ${ }^{334}$ is to assess the safety data of all available human studies on hawthorn monopreparations. Systematic searches were conducted on MEDLINE, EMBASE, AMED, The Cochrane Library, the UK National Research Register and the US ClinicalTrials.gov (up to January 2005). Data were requested from the spontaneous reporting scheme of the WHO. Hand searches were also conducted in a sample of relevant medical journals, conference proceedings, reference lists of identified articles and our own files. Eight manufacturers of hawthorn-containing preparations were contacted and asked to supply any information on adverse events or drug interactions. Data from all clinical studies and reports were assessed. Only human studies on monopreparations were included. Data from hawthorncontaining combination preparations and homeopathic preparations were excluded. All studies were read and evaluated by one reviewer and independently verified by at least one additional reviewer.Twenty-nine clinical studies were identified, of which 24 met our inclusion criteria. A total of 7311 patients were enrolled, and data from 5,577 patients were available for analysis. The daily dose and duration of treatment with hawthorn monopreparations ranged from 160 to $1,800 \mathrm{mg}$ and from 3 to 24 weeks, respectively. The extracts most used in the clinical trials were WS 1,442 (extract of hawthorn standardised to $18.75 \%$ oligomeric procyanidins) and LI 132 (extract of hawthorn standardised to $2.25 \%$ flavonoids). Overall, 166 adverse events were reported. Most of these adverse events were, in general, mild to moderate; eight severe adverse events have been reported with the LI 132 extract. The most frequent adverse events were dizziness/vertigo $(\mathrm{n}=15)$, gastrointestinal complaints $(\mathrm{n}=24)$, headache $(\mathrm{n}=9)$, migraine $(\mathrm{n}=8)$ and palpitation $(\mathrm{n}=11)$. The WHO spontaneous reporting scheme received 18 case reports. In the identified trials, the most frequent adverse events were dizziness $(n=6)$, nausea $(n=5)$, fall $(n=2)$, gastrointestinal haemorrhage $(n=2)$, circulation failure $(n=2)$ and erythematous rash $(n=2)$. There were no reports of drug interactions. In conclusion, all data reviewed in this article seem to indicate that hawthorn is well tolerated even if some severe adverse events were reported; this suggests that further studies are needed to better assess the safety of hawthorn-containing preparations. Moreover, the unsupervised use of this drug can be associated with problems, especially if given with concomitant medications

\subsection{Toxicity of echinacea (1 systematic review)}

Echinacea spp. are native to North America and were traditionally used by the Indian tribes for a variety of ailments, including mouth sores, colds and snakebites. The three most commonly used Echinacea spp. are E. angustifolia, E. pallida and E. purpurea. Systematic literature searches were conducted in six electronic databases and the reference lists of all of the papers located were checked for further relevant publications. ${ }^{335}$ Information was also sought from the spontaneous reporting programmes of the WHO and national drug safety bodies. Twenty-three manufacturers of echinacea were contacted and asked for data held on file. Finally our own departmental files were searched. No language restrictions were imposed. Combination products and homeopathic preparations were excluded. Data from clinical studies and spontaneous reporting programmes suggest that adverse events with echinacea are not commonly reported. Gastrointestinal upsets and rashes occur most frequently. However, in rare cases, echinacea can be associated with allergic reactions that may be severe. Although there is a large amount of data that investigates the efficacy of echinacea, safety issues and the monitoring of adverse events have not been focused on. Shortterm use of echinacea is associated with a relatively good safety profile, with a slight risk of transient, reversible, adverse events. The association of echinacea with allergic reactions is supported by the present evaluation. While these reactions are likely to be rare, patients with allergy 
or asthma should carefully consider their use of echinacea. The use of echinacea products during pregnancy and lactation would appear to be ill-advised in light of the paucity of data in this area.

\subsection{Toxicity of panax ginseng (1 systematic review)}

Panax ginseng C. A. Meyer, a perennial herb native to Korea and China, has been used as an herbal remedy in eastern Asia for thousands of years. Modern therapeutic claims refer to vitality, immune function, cancer, cardiovascular diseases, improvement of cognitive and physical performance and sexual function. A recent systematic review of randomised controlled trials found that the efficacy of ginseng root could not be established beyond doubt for any of these indications. To obtain a balanced assessment of the therapeutic value of $P$. ginseng it is also necessary to consider the safety profile. In view of the extremely widespread use of $P$. ginseng it seems important to ask whether this herbal medicine involves health risks for the consumer. This review was conducted as a systematic attempt to document and evaluate all the available safety data on $P$. ginseng root extracts. Systematic searches were performed in five electronic databases and the reference lists of all papers located were checked for further relevant publications. ${ }^{336}$ All articles containing original data on adverse events and drug interactions with $P$. ginseng were included. Information was also requested from 12 manufacturers of ginseng preparations, the spontaneous reporting schemes of the WHO and national drug safety bodies. No language restrictions were imposed. Data from clinical trials suggest that the incidence of adverse events with ginseng monopreparations is similar to that with placebo. The most commonly experienced adverse events are headache, sleep and gastrointestinal disorders. The possibility of more serious adverse events is indicated in isolated case reports and data from spontaneous reporting schemes; however, causality is often difficult to determine from the evidence provided. Combination products containing ginseng as one of several constituents have been associated with serious adverse events and even fatalities. Interpretation of these cases is difficult as ingredients other than $P$. ginseng may have caused the problems. Possible drug interactions have been reported between $P$. ginseng and warfarin, phenelzine and alcohol.

Collectively, these data suggest that $P$. ginseng monopreparations are rarely associated with adverse events or drug interactions. Those that are documented are usually mild and transient. Combined preparations are more often associated with such events but causal attribution is usually not possible.

\subsection{Toxicity of kava (3 systematic reviews)}

We systematically reviewed the clinical evidence relating to the safety of extracts of the herbal anxiolytic kava (Piper methysticum). ${ }^{337}$ Literature searches were conducted in four electronic databases and the reference lists of all papers located were checked for further relevant publications. Information was also sought from the spontaneous reporting schemes of the World Health Organisation (WHO) and national drug safety bodies, and ten manufacturers of kava preparations were contacted. Data from short-term post-marketing surveillance studies and clinical trials suggest that adverse events are, in general, rare, mild and reversible. However, published case reports indicate that serious adverse events are possible including dermatological reactions, neurological complications and, of greatest concern, liver damage. Spontaneous reporting schemes also suggest that the most common adverse events are mild, but that serious ones occur. Controlled trials suggest that kava extracts do not impair cognitive performance and vigilance or potentiate the effects of central nervous system depressants. However, a possible interaction with benzodiazepines has been reported. It was concluded that when taken as a short-term monotherapy at recommended doses, kava extracts appear to be well tolerated by most users. Serious adverse events have been reported and further research is required to determine the nature and frequency of such events. 
Recently kava has been taken off several national markets because of suspected liver toxic effects. The decision was based on about 70 case reports worldwide, few of which were conclusive. It was argued that the total ban was premature as synthetic anxiolytics are associated with a higher incidence of adverse effects. ${ }^{338}$

Our current knowledge regarding the case reports suggesting hepatotoxic effects of kava was summarised in a brief 'letter to the Editor' in the Lancet. ${ }^{339}$

A further systematic review ${ }^{340}$ discusses the proposed uses, dosing parameters, adverse effects, toxicology, interactions and mechanism of action of kava. The widespread concern regarding the potential hepatoxicity of kava is discussed. A recommendation is made to consolidate and analyses available reports and to continue postmarket surveillance in an international repository to prevent duplicates and promote collection of thorough details at the time of each report so that any association with kava is clearly defined.

\subsection{Toxicity of black cohosh (1 systematic review)}

The aim of this paper was to systematically review the available data relating to the safety of medicinal extracts of black cohosh (Actaea racemosa) ${ }^{341}$ Systematic literature searches were conducted in seven electronic databases, and the reference lists of all papers located were checked for further relevant publications. Information was also sought from the spontaneous reporting programs of the World Health Organisation and national drug safety bodies. Sixteen manufacturers of black cohosh preparations were contacted and asked for data held on file. Finally, our own departmental files were searched. No language restrictions were imposed. Combination products and homoeopathic preparations were excluded. Data from clinical studies and spontaneous reporting programmes suggest that adverse events (AEs) with black cohosh are rare, mild and reversible. Gastrointestinal upsets and rashes are the most common AEs. The spontaneous reporting programmes do contain a few serious AEs, including hepatic and circulatory conditions, but causality cannot be determined. Although there is a large amount of data investigating the efficacy of black cohosh, in particular the product Remifemin, safety issues and the monitoring of AEs have not been the focus. We concluded that if black cohosh products are taken for a limited length of time, there seems to be a slight risk of mild, transient AEs. More serious AEs seem to be rare, and it is impossible to ascertain causality with black cohosh using the limited data available. Thus, although definitive evidence is not available, it would seem that black cohosh is a safe herbal medicine.

\subsection{Toxicity of saw palmetto (1 systematic review)}

Serenoa repens (W. Bartram) Small, also known as saw palmetto, is one of the most widely used herbal preparations for the treatment of lower urinary tract symptoms (LUTS) and benign prostatic hyperplasia (BPH). Although a number of randomized controlled trials (RCTs) and systematic reviews of the efficacy of S. repens for the treatment of LUTS and BPH have been published, no systematic review on its drug interactions or adverse events currently exists. This review ${ }^{342}$ assesses all available human safety data of S. repens monopreparations. Systematic literature searches were conducted from date of inception to February 2008 in five electronic databases; reference lists and our departmental files were checked for further relevant publications. Information was requested from spontaneous reporting schemes of the WHO and national safety bodies. Twenty-four manufacturers/distributors of S. repens preparations and four herbalist organizations were contacted for additional information. No language restrictions were imposed. Only reports of adverse events in humans from monopreparations of S. repens were included. Data from all articles, regardless of study design, reporting adverse events or interactions were independently extracted by the first 
author and validated by the second. Forty articles ( 26 randomized controlled trials, 4 nonrandomized controlled trials, 6 uncontrolled trials and 4 case reports/series) were included. They suggest that adverse events associated with the use of S. repens are mild and similar to those with placebo. The most frequently reported adverse events are abdominal pain, diarrhoea, nausea, fatigue, headache, decreased libido and rhinitis. More serious adverse events such as death and cerebral haemorrhage are reported in isolated case reports and data from spontaneous reporting schemes, but causality is questionable. No drug interactions were reported. Currently available data suggest that $\mathrm{S}$. repens is well tolerated by most users and is not associated with serious adverse events. The majority of adverse events are mild, infrequent and reversible, and include abdominal pain, diarrhoea, nausea and fatigue, headache, decreased libido and rhinitis. We found no evidence for drug interactions with S. repens. However, higher quality reporting of adverse events is essential if safety assessments are to be improved in future.

\subsection{Adverse effect of HMPs in pregnancy (1 systematic review)}

A systematic review ${ }^{343}$ was aimed at summarising the data relating to adverse effects of HMPs during pregnancy. Patients' surveys indicate that many pregnant women try HMPs. Therapists' surveys suggest that this often occurs on the recommendation of healthcare professionals. Case reports and case series show that HMP-use is associated with serious adverse effects. The implicated herbs include:

- blue cohosh

- dong quai

- ginseng

- polygonum multiflorum

- plants containing pyrrolizidine alkaloids

- St John's wort

- tripterygium wilfordii

- montanoa tomentosa

- raspberry leaf

- evening primrose oil.

The frequency of these adverse effects is currently not known.

\subsection{Adverse effects of unconventional therapies in children (1 systematic review)}

The aim of this systematic review was to summarise the recent evidence of adverse effects of CM in children. ${ }^{344}$ Computerised literature searches were carried out in five databases to identify all recent reports of adverse events associated with unconventional therapies in children. The reports were summarised in narrative and tabular form. The results show that numerous case reports and several case series have been published since 1990. However, investigations of a more systematic nature are rare. Most of the adverse events were associated with herbal medications. Inadequately regulated herbal medicines may contain toxic plant material, be contaminated with heavy metals, or be adulterated with synthetic drugs. The adverse events included bradycardia, brain damage, cardiogenic shock, diabetic coma, encephalopathy, heart rupture, intravascular haemolysis, liver failure, respiratory failure, toxic hepatitis and death. A high degree of uncertainty regarding a causal relationship between therapy and adverse event was frequently noted. The size of the problem and its importance relative to the well-documented risks of conventional treatments is currently unknown. Several unconventional therapies may constitute a risk to the health of children and 
adolescents. At present, it is impossible to provide reliable incidence figures. It seems important to be vigilant and investigate this area more systematically.

\subsection{Adverse effects of HMPs in dermatology (1 systematic review)}

Herbal treatments are often used for dermatological conditions. Thus dermatologists should know about their potential to cause adverse events. This review was aimed at addressing this area in a semi-systematic fashion. ${ }^{345}$ Some agents, particularly Chinese herbal creams, have been repeatedly shown to be adulterated with corticosteroids. Virtually all herbal remedies can cause allergic reactions and several can be responsible for photosensitization. Some herbal medicines, in particular Ayurvedic remedies, contain arsenic or mercury that can produce typical skin lesions. Other popular remedies that can cause dermatological side-effects include St John's wort, kava, aloe vera, eucalyptus, camphor, henna and yohimbine. Finally, there are some herbal treatments used specifically for dermatological conditions, e.g. Chinese oral herbal remedies for eczema, which have the potential to cause systemic adverse effects. It was concluded that adverse effects of herbal medicines are an important albeit neglected subject in dermatology, which deserves further systematic investigation.

\subsection{HMPs' influence on blood coagulation (1 systematic review/1 survey)}

This article aimed to summarise our knowledge about the effects of herbal medicinal products (HMPs) on blood coagulation. ${ }^{346}$ Both direct and indirect evidence was found for HMPs to affect the plasmatic coagulation cascade as well as the function of platelets. The HMPs most frequently implicated were danshen (Salvia miltiorrhiza), St John's wort (Hypericum perforatum), dong quai (Angelica sinensis, et al) and ginkgo (Ginkgo biloba). It was concluded that our current knowledge in this area is woefully incomplete. Vis-a-vis the popularity of HMPs, more systematic research is urgently needed.

The ageing population is taking an increasing number of both prescribed and non-prescribed medication. Little is known of the potential for adverse drug reactions between these. Warfarin is a commonly prescribed medication, well known for its potential to cause serious adverse reactions in combination with many prescription medicines. It has been suggested that herbal medicines such as garlic, either as a dietary supplement or in cooking, may also interact with warfarin, resulting in poor international normalised ratio (INR) control. The aim of this retrospective study ${ }^{347}$ was to determine whether, for patients who take garlic as well as warfarin, the proportion of the INR tests in range is lower than in comparable patients who do not take garlic. Three controls (not taking garlic) matched for age, sex, and general practice were compared with each patient self-reporting taking garlic as a supplement. INR results were assessed for the preceding 12 months. Potentially confounding factors were considered, for example diabetes mellitus; all prescribed medication; any bleeding episodes. No evidence was found to suggest that garlic consumption either as a supplement or in cooking is associated with more frequent haemorrhagic complications or less control of INR. Poor INR control may, however, be associated with taking larger numbers of prescription medicines, particularly during prescription changes. Further research would be warranted into whether increased INR monitoring is needed when drug changes are made. These data render clinically significant interactions between warfarin and garlic intake unlikely.

\subsection{Herb-drug interactions ( 8 systematic reviews/1 editorial/2 surveys)}

Herbal medicines are becoming more and more popular. The safety aspects of this widespread use are under-researched. This applies in particular to the possibility of herb/drug interactions. This 
systematic review summarises ${ }^{348}$ the indirect published evidence on this topic. Its results show that numerous suggestions about such interactions exist. Serious doubts, however, remain as to the reliability of this information. The implications of herb/drug interactions are potentially serious. Therefore, more rigorous research is urgently required.

A further article ${ }^{349}$ represents an attempt to summarise anecdotal and experimental evidence regarding such interactions. Four independent literature searches were carried out to identify case reports and systematic clinical investigations of herb/drug interactions. Numerous case reports of herb/drug interactions were found. Several of these relate to interactions of herbal remedies with synthetic anticoagulants. Such anecdotal evidence, however, has its obvious limitations. Systematic investigations, which might overcome these limitations, are still extremely sparse. It is concluded that interactions between herbal medicines and synthetic drugs undoubtedly exist. Yet, at present, our knowledge of the subject is incomplete. In the interest of consumer safety, this area deserves more rigorous research. This review has recently been updated. ${ }^{350}$

A systematic review summarised all relevant clinical cases on the subject of St John's wort interactions with cyclosporine. ${ }^{351}$ Five independent computerised literature searches were conducted to identify all reports of such interactions. Data were extracted and are summarised in narrative form. Eleven case reports and 2 case series were located. In most instances, causality between St John's wort and the clinical or biochemical result is well established. The mechanism of interaction between St John's wort and cyclosporine has recently been elucidated and involves both Pglycoprotein and cytochrome P4503A4 expression. Collectively these data leave little doubt that St john's wort interacts with cyclosporine, causing a decrease of cyclosporine blood levels and leading in several cases to transplant rejection. It was concluded that St John's wort can endanger the success of organ transplantations. Adequate information may be the best way to avoid future incidences.

An Editorial also addressed the subject of herb-drug interactions. ${ }^{352}$ It reported interactions related to Ginkgo biloba, St John's wort, Panax ginseng, Garlic, Echinacea, Saw palmetto and Kava. It also asked why have only so few cases of suspected herb-drug interactions been reported in the medical literature? One answer might be that they are truly rare events. Another answer lies in underreporting. The declaration of known adverse effects is often disappointingly low. The reporting of clinical events that have no reasonable explanation (because we quite simply are not aware of a plausible mechanism) must be close to zero. Who would, for instance, have thought of an interaction between St John's wort and warfarin 1 year ago if an international normalised ratio reading had yielded an unexpected result? Which healthcare professional would even be aware that their patient has decided to self-medicate St Johns' wort for a spell of low mood? And who today would know of the emerging evidence that St John's wort interferes with the metabolism or elimination of oral anti-coagulants and other drugs?

The aim of this survey ${ }^{353}$ was to estimate the prevalence of the use of herbal medicines by patients taking warfarin (co-ingestion). Thirty-five general practices in Devon and Somerset identified 2600 patients taking warfarin and sent postal questionnaires to them. One thousand, three hundred and sixty usable responses were received (response rate $=54.2 \%$ ). One or more of the specified herbal remedies thought to interact with warfarin were taken by $8.8 \%$ of all patients. Complementary or homoeopathic treatments not specified in the survey questionnaire were taken by $14.3 \%$ of responders. Overall, $19.2 \%$ of responders were taking one or more such medicines. The use of herbal medicines had not been discussed with a conventional healthcare professional by $92.2 \%$ of patients. Twenty-eight point three per cent of responders thought that herbal medicines might or definitely could interfere with other drugs prescribed by their doctor, however, patients taking any 
non-prescribed medication were less likely to believe this $\left(\chi^{2}=20\right.$, degrees of freedom $=1$, $\mathrm{P}<0.001)$.

We also published a systematic review ${ }^{354}$ of the literature to determine the possible interactions between the seven top-selling herbal medicines (ginkgo, St John's wort, ginseng, garlic, echinacea, saw palmetto and kava) and prescribed drugs. Literature searches were performed using the following databases: MEDLINE (via Pubmed), Cochrane Library, EMBASE and Phytobase (all from their inception to July 2000). All data relating to herb-drug interactions were included regardless of whether they were based on case reports, case series, clinical trials or other types of investigation in humans. In vitro experiments were excluded. Data were extracted by the first author and validated by the second author. 41 case reports or case series and 17 clinical trials were identified. The results indicate that St John's wort (Hypericum perforatum) lowers blood concentrations of cyclosporin, amitriptyline, digoxin, indinavir, warfarin, phenprocoumon and theophylline; furthermore, it causes intermenstrual bleeding, delirium or mild serotonin syndrome, respectively, when used concomitantly with oral contraceptives (ethinylestradiol/desogestrel), loperamide or selective serotonin-reuptake inhibitors (sertraline, paroxetine, nefazodone). Ginkgo (Ginkgo biloba) interactions include bleeding when combined with warfarin, raised blood pressure when combined with a thiazide diuretic and coma when combined with trazodone. Ginseng (Panax ginseng) lowers blood concentrations of alcohol and warfarin, and induces mania if used concomitantly with phenelzine. Garlic (Allium sativum) changes pharmacokinetic variables of paracetamol, decreases blood concentrations of warfarin and produces hypoglycaemia when taken with chlorpropamide. Kava (Piper methysticum) increases 'off' periods in Parkinson's disease patients taking levodopa and can cause a semicomatose state when given concomitantly with alprazolam. No interactions were found for echinacea (Echinacea augustifolia, E. purpurea, E. pallida) and saw palmetto (Serenoa repens).

In spite of the paucity of reliable information available to date, the message for healthcare professionals seems clear: at the very least we should routinely include questions relating to the usage of herbal medicines in our history taking. The message for herbal manufacturers is trickier but equally obvious: interactions have to be systematically investigated and the results of this research must be made known widely and swiftly. If handled irresponsibly, the subject of herb-drug interaction might turn the 'herbal boom' into a 'herbal nightmare' for those responsible. The message for licensing authorities relates to the safety of herbal medicinal products in more general terms: lack of evidence of risks is clearly not the same as evidence of lack of risks. It may be time to reconsider the regulatory status of herbal food supplements with powerful pharmacological actions that have the potential for benefit and risk.

The leading 44 pharmaceutical/herbal companies worldwide received questionnaires: 15 (34\%) questionnaires were completed and retuned; ${ }^{355} 5$ companies $(11 \%)$ replied but did not complete the questionnaire; and 24 companies (54\%) failed to respond. Of the 15 responders, $10(67 \%)$ considered herb-drug interactions to be an important issue, but only $3(20 \%)$ conducted studies, and only $2(13 \%)$ regularly allocated funds to such research. Two herbal companies indicated that $5 \%$ and $6 \%$ respectively of their annual research and development budget was allocated to research herb-drug interactions in 2000. They were the only companies to address this question. These findings suggest that there is a widespread lack of interest in herb-drug interactions within the pharmaceutical and herbal industries and highlight the lack of research currently being undertaken. Knowing that millions of patients take herbal and conventional medicine concomitantly, often without the knowledge of their physicians, and considering our current lack of understanding of herb-drug interactions, more systematic research into this area seems a matter of urgency. 
Use of herbal medicines among patients under cardiovascular pharmacotherapy is widespread. In this systematic review, ${ }^{356}$ we have reviewed the literature to determine the possible interactions between herbal medicines and cardiovascular drugs. The Medline database was searched for clinical articles published between January 1996 and February 2003. Forty-three case reports and eight clinical trials were identified. Warfarin was the most common cardiovascular drug involved. It was found to interact with boldo, curbicin, fenugreek, garlic, danshen, devil's claw, don quai, ginkgo, papaya, lycium, mango, PC-SPES (resulting in over-anticoagulation) and with ginseng, green tea, soy and St. John's wort (causing decreased anticoagulant effect). Gum guar, St. John's wort, Siberian ginseng and wheat bran were found to decrease plasma digoxin concentration; aspirin interactions include spontaneous hyphema when associated with ginkgo and increased bioavailability if combined with tamarind. Decreased plasma concentration of simvastatin or lovastatin was observed after co-administration with St. John's wort and wheat bran, respectively. Other adverse events include hypertension after co-administration of ginkgo and a diuretic thiazide, hypokalemia after liquorice and antihypertensives and anticoagulation after phenprocoumon and St. John's wort. Interaction between herbal medicine and cardiovascular drugs is a potentially important safety issue. Patients taking anticoagulants are at the highest risk.

Herbal medicine is one of the most popular choices of complementary therapies for women, particularly as an alternative treatment for menopausal symptoms. ${ }^{357}$ The most commonly used herbal medicines for the menopause is probably black cohosh (Actaea/Cimcifuga racemosa); other preparations used include red clover (Trifolium pretense), dong quai (Angelica sinesis) and evening primrose (Oemothera biennis). Some of these herbal medicines have a very good safety profile with little or no suggestion of interaction with conventional drugs. For others, there are many and significant drug-herb interactions of herbal medicines thought to be of benefit for menopausal symptoms, as well as discussing the implications for the medical profession.

For an update of this article ${ }^{358}$, we have reviewed the literature to determine the possible interactions between seven popular herbal medicines (ginkgo, St John's wort, ginseng, garlic, echinacea, saw palmetto and kava) and conventional drugs. Literature searches were performed using MEDLINE, Cochrane Library and EMBASE and we identified 128 case reports or case series, and 80 clinical trials. Clinical trials indicate that St John's wort (Hypericum perforatum), via cytochrome P450 (CYP) and/or P-glycoprotein induction, reduces the plasma concentrations (and/or increases the clearance) of alprazolam, amitriptyline, atorvastatin, chlorzoxazone, ciclosporin, debrisoquine, digoxin, erythromycin, fexofenadine, gliclazide, imatinib, indinavir, irinotecan, ivabradine, mephenytoin, methadone, midazolam, nifedipine, omeprazole, oral contraceptives, quazepam, simvastatin, tacrolimus, talinolol, verapamil, voriconazole and warfarin. Case reports or case series suggest interactions of St John's wort with adrenergic vasopressors, anaesthetics, bupropion, buspirone, ciclosporin, eletriptan, loperamide, nefazodone, nevirapine, oral contraceptives, paroxetine, phenprocoumon, prednisone, sertraline, tacrolimus, theophylline, tibolone, tryptophan, venlafaxine and warfarin. Ginkgo (Ginkgo biloba) decreases the plasma concentrations of omeprazole, ritonavir and tolbutamide. Clinical cases indicate interactions of ginkgo with antiepileptics, aspirin (acetylsalicylic acid), diuretics, ibuprofen, risperidone, rofecoxib, trazodone and warfarin. Ginseng (Panax ginseng) may interact with phenelzine and warfarin. Kava (Piper methysticum) increases the clearance of chlorzoxazone (a CYP2E1 substrate) and may interact with alprazolam, levodopa and paroxetine. Garlic (Allium sativum) interacts with chlorpropamide, fluindione, ritonavir and warfarin; it also reduces plasma concentrations of chlorzoxazone (a CYP2E1 probe). Echinacea might affect the clearance of caffeine (a CYP1A2 probe) and midazolam (a CYP3A4 probe). No interactions have been reported for saw palmetto (Serenoa repens). Numerous interactions between herbal medicines and conventional drugs have been documented. While the significance of many interactions is uncertain, several interactions, particularly those with St John's wort, may have serious clinical consequences. 
A systematic literature review ${ }^{359}$ was conducted to investigate the effects of Ginkgo biloba preparations on blood coagulation parameters. Systematic literature searches were carried out using MEDLINE (via PubMed), EMBASE, AMED, CINAHL $\square$, the Cochrane Library and PsycINFO to identify randomised, double-blind, clinical trials of $\mathrm{G}$. biloba mono-preparations assessing at least one blood coagulation parameter as either a primary or a supplementary outcome measure. Five manufacturers of G. biloba preparations were asked to contribute any unpublished material. Data were validated and extracted independently by two reviewers according to pre-specified criteria. Eight trials were included, of which two included healthy subjects, four included patients with various conditions and two included participants taking concomitant anticoagulant medication, one with acetylsalicylic acid (ASA; aspirin) and one with warfarin. Overall results do not provide evidence that $\mathrm{G}$. biloba does affect blood coagulation parameters in a clinically relevant manner. Out of many parameters assessed, four coagulation parameters from three studies were significantly different in the treatment group compared with the control. One study comparing the treatments of ASA and G. biloba with ASA alone confirmed that the two treatments were equivalent with regards to all but three coagulation parameters. Available evidence does not demonstrate that extract of G. biloba causes significant changes in blood coagulation parameters. A limited number of studies on concomitant use of G. biloba with ASA or warfarin also do not suggest that G. biloba has an additive effect to the clinical effects of these two drugs. Currently available evidence is limited and, therefore, further studies are needed to assess effects of G. biloba on a variety of blood coagulation parameters.

\subsection{Contamination of HMPs (2 systematic reviews)}

The growing popularity of traditional Indian remedies necessitates a critical evaluation of risks associated with their use. This systematic review ${ }^{360}$ summarised all available data relating to the heavy metal content in such remedies. Computerised literature searches were carried out to identify all articles with original data on this subject. Fifteen case reports and six case series were found. Their collective results suggest that heavy metals, particularly lead, have been a regular constituent of traditional Indian remedies. This has repeatedly caused serious harm to patients taking such remedies. The incidence of heavy metal contamination is not known, but one study shows $64 \%$ of samples collected in India contained significant amounts of lead (64\% mercury, $41 \%$ arsenic and $9 \%$ cadmium). These findings should alert us to the possibility of heavy metal content in traditional Indian remedies and motivate us to consider means of protecting consumers from such risks.

A similar approach was used to review the evidence of heavy metal contamination in Chinese herbal medicines. ${ }^{361}$ Twenty-two publications were located. The most frequently found contaminant was lead. In several cases serious health consequences were the result of administration of such remedies.

\subsection{Adulteration of HMPs (1 systematic review)}

The popularity of Chinese herbal medicines (CHMs) demands a critical analysis of safety issues. The aim of this systematic review was to summarise data regarding adulterations of CHMs with conventional drugs. ${ }^{362}$ Literature searches were carried out in six databases. Articles containing original data on adulterations were considered without language restrictions. Eighteen case reports, two case series and four analytical investigations were identified. The list of adulterants contains drugs associated with serious adverse effects like corticosteroids. In several instances, patients were seriously harmed. One report from Taiwan suggests that $24 \%$ of all samples were contaminated with at least one conventional pharmacological compound. It is concluded that adulteration of CHMs 
with synthetic drugs is a potentially serious problem which needs to be addressed by adequate regulatory measures.

\subsection{Herbal mixtures (1 post-marketing surveillance study)}

Our knowledge relating to adverse drug reactions (ADRs) of phytomedicines is highly fragmentary. The aim of this study ${ }^{363}$ was to define the prevalence of ADRs following medication with herbal or synthetic expectorants. In a multicentre, comparative post-marketing surveillance study of more than 3000 patients with acute bronchitis, about half were treated with a herbal remedy Sinupret ${ }^{\circledR}$, and the other half with various other expectorants. In ascending order of incidence, ADRs were noted during mono-medication of Sinupret ${ }^{\circledR}(0.8 \%)$, Ambroxol $(1.0 \%)$ and acetylcysteine (4.3\%). When concomitant drugs were used, this rank order was unchanged but incidence rates were markedly increased (3.4, 6.5 and 8.2\% respectively). The most frequent ADRs were gastrointestinal problems. It is concluded that expectorants are associated with ADRs in roughly $1-5 \%$ of cases undergoing single drug treatment and in 3-10\% when more than one medication is being used. Amongst the expectorants used in this study, the herbal preparation Sinupret ${ }^{\circledR}$ is associated with the lowest incidence of ADRs.

\subsection{St John's wort (3 systematic reviews)}

A further aim was to provide a systematic review ${ }^{364}$ of ADRs associated with the use of extracts of the herb St. John's wort (Hypericum perforatum L.) for the treatment of mild to moderate depression. Searches of four computerised databases were performed for records of ADRs. Manufacturers of hypericum products, the international drug monitoring centre of the World Health Organisation (WHO) and the national drug safety monitoring bodies of Germany and the United Kingdom were also contacted for information. Information on ADRs originates from case reports, clinical trials, post-marketing surveillance and drug monitoring studies. Collectively, the data suggest that hypericum is well tolerated, with an incidence of adverse reactions similar to that of placebo. The most common adverse effects are gastrointestinal symptoms, dizziness/confusion and tiredness/sedation. A potential serious adverse effect is photosensitivity, but this appears to occur extremely rarely. Hypericum has an encouraging safety profile. However, as most of the current data originate from short-term investigations, more long-term studies are desirable.

Conventional antidepressant drugs are associated with a range of ADRs. The herb $\underline{\text { St. John's wort }}$ has been shown to be useful for treating mild to moderate depression and has been associated with few ADRs. In an attempt to systematically compare safety data for hypericum with those of several conventional antidepressants, data were obtained from reports of the clinical trial programmes of the following drugs: dothiepin, fluoxetine, moclobemide and mirtazapine. ${ }^{365}$ Although hypericum was associated with fewer and milder ADRs in clinical trials than any of the other drugs, comparisons were difficult because of the different forms in which information was presented. Data from spontaneous reporting schemes and drug monitoring studies have also suggested that ADRs due to hypericum are rare and mild, although there has been one recent report of a possible serious ADR with hypericum. Data on the safety of hypericum in overdose and on interactions with other drugs are as yet scarce.

St John's wort (SJW) has recently been associated with psychotic events. We therefore systematically reviewed the clinical evidence associating SJW with psychotic events. ${ }^{366}$ Five electronic databases were searched for all relevant reports. Data were extracted according to predefined criteria. Seventeen case reports associated the use of SJW with psychotic events. In 12 instances, the diagnosis was mania or hypomania. Causality is in most cases possible. In no case 
was a positive rechallenge reported. These case reports raise the possibility that SJW may trigger episodes of mania in vulnerable patients. Clinicians should be aware of this possibility and researchers should attempt to ascertain whether the association is causal.

\subsection{Acupuncture (1 clinical trial/11 systematic reviews)}

Acupuncture is amongst the oldest forms of medical treatment. Many proponents feel that this fact sufficiently demonstrates its safety. While traditional use and acceptance renders frequent adverse reactions unlikely, we must insist on scientific rather than historical proof, particularly for rare yet serious events. In particular, the present popularity of acupuncture and its general acceptance both with patients and doctors necessitates a systematic investigation of safety aspects. A review ${ }^{367}$ summarised the published evidence on serious, potentially life-threatening complications allegedly caused by acupuncture. Its results suggested that serious complications of acupuncture exist. However, they seem to be rarities. Further study of this subject is warranted.

Many Japanese cases of adverse events after acupuncture are not listed in medical databases such as MEDLINE. ${ }^{368}$ Therefore, they are not easily accessible to researchers outside Japan. We therefore wanted to complement existing reviews of adverse events after acupuncture in the West and to provide more detailed discussion and analysis. Literature searches were carried out using Igaku Chuo Zasshi (Japana Centra Revuo Medicina) CD-ROM version covering the period 1987-1999. Case reports of adverse events, which were suspected to be due to acupuncture treatment, were included. Experimental studies, surveys, and news articles were excluded. Two independent reviewers extracted data from located articles in a pre-defined structured way, and assessed the likelihood of causality in each case. Eighty-nine articles which reported 124 cases were located.

The most frequent adverse events were: pneumothorax (25 cases), spinal cord injury (18 cases), acute hepatitis B (11 cases), and localised argyria (10 cases). There were two fatalities from infections. Forty-eight events were caused by needle breakage including 26 cases of intentionally embedded needle and 16 cases of accidental breakage. There were also 10 cases of injury from selftreatment. These results suggest that the training system for acupuncturists (including medical doctors) should be improved and that unsupervised self-treatment should be discouraged.

The aim of another paper was to review the traumatic injuries that have been associated with acupuncture and to discuss how these adverse effects may be reduced by increased awareness of normal anatomy and anatomical variations. ${ }^{369}$ A literature search was carried out accompanied by post-mortem anatomical studies. Traumatic lesions after acupuncture have been described in thoracic and abdominal viscera, in the peripheral and central nervous systems, and in blood vessels. Deaths have been recorded from pneumothorax and cardiac tamponade. The anatomical structure of the body at several acupuncture points is such that needles can reach vulnerable structures. While the frequency of adverse effects of acupuncture is unknown and they may be rare, knowledge of normal anatomy and anatomical variations is essential for safe practice and should be reviewed by regulatory bodies and those responsible for training courses.

As retrospective data has obvious limitations, a systematic review ${ }^{370}$ was conducted of all prospective studies of this area. A search for prospective surveys of the safety of acupuncture was conducted using computerised databases (MEDLINE, EMBASE, the Cochrane Library, and CISCOM), enquiries to acupuncture organisations and our own files. Data on sample, size, types of patients, duration of study, types of acupuncture, definition of adverse events, method of evaluation, and findings were systematically extracted from the retrieved reports. Nine surveys were located and included in the review. Their results were not uniform. The most common adverse events were needle pain ( $1 \%$ to $45 \%)$ from treatments, tiredness ( $2 \%$ to $41 \%)$, and bleeding $(0.03 \%$ to $38 \%)$. 
Feelings of faintness and syncope were uncommon, with an incidence of $0 \%$ to $0.3 \%$. Feelings of relaxation were reported by as many as $86 \%$ of patients. Pneumothorax was rare, occurring only twice in nearly a quarter of a million treatments. It was concluded that, although the incidence of minor adverse events associated with acupuncture may be considerable, serious adverse events are rare. Those responsible for establishing competence in acupuncture should consider how to reduce these risks.

A prospective study was undertaken using forms for intensive event monitoring that had been previously piloted. ${ }^{371}$ Minor adverse events were defined as "any ill-effect, no matter how small, that is unintended and non-therapeutic, even if not unexpected". These events were reported every month, along with the total number of consultations. Minor or serious events were reported on separate forms when they occurred. A sample size of 30,000 consultations was necessary to identify with $95 \%$ confidence any adverse event with a frequency of 1 in 10,000 consultations. Estimates of incidences per 10,000 population were calculated with the acupuncturist (not the consultation) as the primary sampling unit. Data were collected from June 1998 to February 2000 from 78 acupuncturists, 13 of whom chose to remain anonymous. The average age of the acupuncturists was 47 (range $27-71$ ) years, $61 \%$ were doctors and 39\% physiotherapists, and $71 \%$ had practised for five years or more. In all, 31,822 (median 318, range 5-1911) consultations were included. Altogether, 43 "significant" events were reported, giving a rate of 14 per 10,000 (95\% confidence interval 8/10,000 to 20/10,000). In addition, 48 apparently similar events were reported on the monthly forms, presumably due to different interpretations of "significant". All adverse events had cleared within one week, except for one incident of pain that lasted two weeks and one of sensory symptoms that lasted several weeks. According to accepted criteria, none $(0 / 10,000$ to $1.2 / 10,000)$ of these events was serious. A total of 2,135 minor events was reported, giving an incidence of 671 per $10,000(42 / 10,000$ to $1013 / 10,000)$ consultations. The most common events were bleeding (160 to 590) per 10,000 consultations) and needling pain (110 (49-247) per 10,000 consultations). Aggravation of symptoms occurred in $96(43-178)$ per 10,000 consultations; in $70 \%$ of these cases, there was a subsequent improvement in the presenting complaint. The highest rates reported by individual acupuncturists, expressed as a percentage of consultations, were $53 \%$ for bleeding, $24 \%$ for pain, and $11 \%$ for aggravation of symptoms. Because these data were published as a short report, important methodological details had to be omitted. We therefore decided to publish a full account elsewhere. ${ }^{372}$

Even though acupuncture is safe as used in the West, it might still be associated with risks as employed in developing countries. In particular, the risk of hepatitis infection through poor hygiene is of concern. The aim of this systematic review ${ }^{373}$ was therefore to critically evaluate such data from epidemiological investigations. Four independent literature searches were carried out to identify all epidemiological evidence linking acupuncture with hepatitis. All studies were validated by the authors and data extracted according to predefined criteria. Fifteen investigations fulfilled our inclusion criteria. Most studies originated from Asia. One study included markers to hepatitis A virus, three to hepatitis $\mathrm{B}$ virus, and 13 to hepatitis $\mathrm{C}$ virus. Five investigations reported associations between acupuncture and seropositivity to hepatitis $\mathrm{C}$ virus. In those studies, acupuncture increased the risk only modestly. It was therefore concluded that a modest association between hepatitis $\mathrm{C}$ and acupuncture exists in some countries. This emphasises the importance of exclusively using disposable acupuncture needles.

Another systematic review ${ }^{374}$ aimed to summarize all reported cases of cardiac tamponade after acupuncture. Five electronic databases and our own files were searched for reports of cardiac tamponade after acupuncture. No restrictions in time or language were imposed. Data were extracted by two independent reviewers according to predefined criteria. We found a total of 26 cases. In 14 patients, the complications were fatal. In most instances, there is little doubt about 
causality. We conclude that cardiac tamponade is a serious, often fatal complication after acupuncture. As it is theoretically avoidable, acupuncturists should be trained to minimize the risk

The objective of this systematic review ${ }^{375}$ was to systematically review the Chinese-language literature on acupuncture-related adverse events. We searched three Chinese databases (the Chinese Biomedical Literature Database, 1980-2009; the Chinese Journal Full-Text Database, 1980-2009; and the Weipu Journal Database, 1989-2009) to identify Chinese-language articles about the safety of traditional needle acupuncture. Case reports, case series, surveys and other observational studies were included if they reported factual data, but review articles, translations and clinical trials were excluded. The inclusion criteria were met by 115 articles (98 case reports and 17 case series) that in total reported on 479 cases of adverse events after acupuncture. Fourteen patients died.

Acupuncture-related adverse events were classified into three categories: traumatic, infectious and "other". The most frequent adverse events were pneumothorax, fainting, subarachnoid haemorrhage and infection, while the most serious ones were cardiovascular injuries, subarachnoid haemorrhage, pneumothorax and recurrent cerebral haemorrhage. Many acupuncture-related adverse events, most of them owing to improper technique, have been described in the published Chinese literature. Efforts should be made to find effective ways of monitoring and minimizing the risks related to acupuncture

Another systematic review ${ }^{376}$ is aimed at summarizing these reports and putting them into a broader context. Four electronic databases and my own files were searched for such reports. No restrictions in terms of language or time were imposed. Case reports, case series and other types of articles reporting fatalities were considered. Key data were extracted according to pre-defined criteria. Reports of 86 deaths after acupuncture were found. Many are incomplete and causality may therefore be occasionally uncertain. Due to under-reporting, these reports are likely to merely describe the tip of a larger iceberg. It was concluded that acupuncture has been associated with numerous deaths. These fatalities are avoidable and a reminder of the need to insist on adequate training for all acupuncturists.

Acupuncture is usually deemed to be as a safe intervention. Yet serious complications continue to be reported. The aim of this article ${ }^{377}$ is to systematically review all serious adverse effects recently reported in the medical literature. Electronic literature searches were conducted to identify case reports, case series, epidemiological studies and other investigations reporting adverse effects published between 2000 and 2009. Data were extracted and categorized according to pre-defined criteria. Results: Numerous reports of severe adverse effects, including those involving the cardiovascular system were found. Many lacked important detail. The adverse effects fall into two major categories: infections and trauma. In most cases, patients made a full recovery but at least 9 patients died and many more suffered permanent problems. Most adverse effects were preventable. Serious adverse effects of acupuncture continue to be reported with some regularity. The majority would seem to be avoidable, and acupuncturists across the world should receive adequate training to minimize the risk.

\subsection{Chelation (1 systematic review)}

Chelation therapy with EDTA us used by alternative practitioners against arteriosclerotic diseases. This systematic review ${ }^{378}$ summarizes the deaths that have been associated with it since 1990 . Extensive literature searches located 1105 articles of which 6 met the inclusion criteria. They reported 9 fatalities. The quality of these reports was too poor to establish causality beyond doubt. It is concluded that EDTA chelation continues to be associated with fatal outcomes. 


\subsection{Spinal manipulation (1 survey/8 systematic reviews)}

To obtain preliminary data on neurological complications of spinal manipulation in the UK all members of the Association of British Neurologists were asked to report cases referred to them of neurological complications occurring within 24 hours of cervical spine manipulation over a 12month period. ${ }^{379}$ The response rate was $74 \%$. Twenty-four respondents reported at least one case each, contributing to a total of about 35 cases. These included 7 cases of stroke in brain stem territory (4 with confirmation of vertebral artery dissection), 2 cases of stroke in carotid territory and 1 case of acute subdural haematoma. There were 3 cases of myelopathy and 3 of cervical radiculopathy. Concern about neurological complications following cervical spine manipulation appears to be justified. A large long-term prospective study is required to determine the scale of the hazard.

The safety of chiropractic is an important, albeit neglected issue. Recent case reports of adverse events were summarised in a recent article. ${ }^{380}$ Spinal manipulation has recently been associated with dissection of the vertebral and internal carotid artery, resulting in strokes and at least one death. Other recent instances relate to epidermal hematoma, intracranial aneurysm, cauda equina syndrome, contusion of the spinal cord, myelopathy and radiculopathy, as well as palsy of the long thoracic nerve. Case series from the Ukraine, Sweden, and Germany have contributed 49, 21, and 10 further patients, respectively, who suffered life-threatening events after spinal manipulation. Retrospective surveys carried out with doctors in California, Ireland and Great Britain brought a total of 145 further case series to light that would otherwise not have been documented in the medical literature.

The aim of a further systematic review ${ }^{381}$ was to summarise the data of all prospective investigations into the safety of spinal manipulation. Five independent literature searches were carried out to identify all such studies. Data were extracted and validated according to pre-defined criteria. Five investigations met the inclusion criteria. The most valid studies suggested that about half of all patients experienced adverse events after chiropractic spinal manipulation. These events were usually mild and transient. No reliable data existed about the incidence of serious adverse events. These data indicated that mild and transient adverse events seem to be frequent. Serious adverse events are probably rare but their incidence can only be estimated at present. Further prospective investigations are needed to define their incidence more closely.

Another systematic review ${ }^{382}$ summarised the evidence about the risks of spinal manipulation. Articles were located through searching three electronic databases (MEDLINE, EMBASE, Cochrane Library), contacting experts $(\mathrm{n}=9)$, scanning reference lists of relevant articles, and searching departmental files. Reports in any language containing data relating to risks associated with spinal manipulation were included, irrespective of the profession of the therapist. Where available, systematic reviews were used as the basis of this article. All papers were evaluated independently by the authors. Data from prospective studies suggest that minor, transient adverse events occur in approximately half of all patients receiving spinal manipulation. The most common serious adverse events are vertebrobasilar accidents, disk herniation, and cauda equina syndrome. Estimates of the incidence of serious complications range from 1 per 2 million manipulations to 1 per 400,000. Given the popularity of spinal manipulation, its safety requires rigorous investigation.

The aim of this systematic review ${ }^{383}$ was to summarise the recent $(1995$ - 2003) evidence on cerebrovascular complications associated with spinal manipulation. Computerized literature searches were carried out without language restrictions. Only articles with original data pertaining to the cerebrovascular risks of spinal manipulation were included. Numerous case reports, case series, retrospective investigations and prospective studies were located. In total more than 300 patients 
were affected. The most frequently reported complication was stroke due to arterial dissection after cervical spinal manipulation. Considering the popularity of spinal manipulative therapies, the overall incidence of such complications is probably low, however, no reliable figures can be generated through this or any other data available to date. It is concluded that serious cerebrovascular complications of spinal manipulation continue to be reported. Their incidence is unknown. Large and rigorous prospective studies are necessary in order to accurately define the risks of spinal manipulation.

The objective of a further systematic review ${ }^{384}$ was to summarise recent evidence from case reports (published January 1995 - September 2001) of adverse events after cervical spine manipulation. Five computerised literature searches (MEDLINE - Pubmed; EMBASE, the Cochrane Library, AMED, and CISCOM) were performed. No language restrictions were applied. All case reports containing original data of adverse events after cervical spine manipulation were included. All articles were evaluated and key data extracted according to pre-defined criteria: patient's age, sex and diagnosis; type of therapist; type of treatment; nature of adverse event; method of diagnosis; and clinical outcome. Thirty-one case reports (42 individual cases) were found. The patients were equally distributed between the sexes ( 21 male, 20 female, one unknown) and mostly middle-aged (range, 3 months to 87 years). Most were treated by chiropractors. Arterial dissection causing stroke was reported in at least 18 cases. It was concluded that serious adverse events after cervical spine manipulation continue to be reported. As the incidence of these events is unknown, large and rigorous prospective studies of cervical spine manipulation are needed to accurately define the risks.

In addition to direct risks associated with the intervention, such as bone fracture due to spinal manipulation, there may be indirect risks associated with chiropractic. Indirect risks are related to the 'philosophy' and clinical practice of a given 'school'. This article ${ }^{385}$ focused on one potential indirect risk of chiropractic, namely chiropractors' use of X-rays. Computerised literature searches were used to access studies of the use of X-rays within the chiropractic profession. The bibliographies of all studies and reviews located were scanned for further relevant reports. Publications were included in this review if they contained factual information on the usage of Xrays by chiropractors. The results suggest that significant parts of the chiropractic profession indulge in an overt over-use of $\mathrm{x}$-ray diagnosis. This has considerable potential for harming the patient.

A review addressed the attitudes of some providers of CM towards immunisation. ${ }^{386}$ Its results indicate that in particular significant proportions of non-medically qualified homoeopaths and some chiropractors warn their patients against any immunisation. This habit has the potential of doing considerable harm both on an individual as well as on a population basis.

An attempt was made to conduct a risk-benefit analysis of chiropractic care. ${ }^{387}$ This showed that benefits are less certain than most like to believe. On the other hand, there are frequent non-serious risks and serious risks of unknown frequency. The conclusion therefore was that chiropractic does not demonstrably do more good than harm.

Upper spinal manipulation (USM) is frequently used by chiropractors and other health care professionals to treat minor complaints. This systematic review ${ }^{388}$ aimed to summarize ophthalmological adverse effects of USM recently reported in the medical literature. Five electronic databases were searched for all case reports of ophthalmological adverse effects after USM published between January 1995 and April 2003. No language restrictions were applied. Key data from the primary publications thus located were extracted and critically evaluated. Fourteen case reports were found. Clinical symptoms and signs were diverse and included loss of vision, ophthalmoplegia, diplopia and Horner's syndrome. The underlying mechanism was arterial wall dissection in most cases. The eventual outcome varied and often included permanent deficits. 
Causality was frequently deemed likely or certain. It was concluded that upper spinal manipulation is associated with ophthalmological adverse effects of unknown frequency. Ophthalmologists should be aware of its risks. Rigorous investigations must be conducted to establish reliable incidence figures.

The objective of this systematic review was to identify adverse effects of spinal manipulation. ${ }^{389}$ All reports of adverse effects published between January 2001 and June 2006 were included. There were no restrictions according to language of publication or research design of the reports. The searches identified 32 case reports, four case series, two prospective series, three case-control studies and three surveys. In case reports or case series, more than 200 patients were suspected to have been seriously harmed. The most common serious adverse effects were due to vertebral artery dissections. The two prospective reports suggested that relatively mild adverse effects occur in $30 \%$ to $61 \%$ of all patients. The case-control studies suggested a causal relationship between spinal manipulation and the adverse effect. The survey data indicated that even serious adverse effects are rarely reported in the medical literature. Spinal manipulation, particularly when performed on the upper spine, is frequently associated with mild to moderate adverse effects. It can also result in serious complications such as vertebral artery dissection followed by stroke. Currently, the incidence of such events is not known. In the interest of patient safety we should reconsider our policy towards the routine use of spinal manipulation.

The aim of this systematic review ${ }^{390}$ was to summarise adverse effects after spinal manipulation reported in the South Korean literature. The Korean literature was searched for relevant articles. Twelve articles met the inclusion criteria. They reported a total of 18 cases. In most instances, the patient made a full recovery. The implicated treatment was frequently a spinal manipulation technique that is an integral part of traditional Korean medicine. It is concluded that adverse effects after spinal manipulation are not specific to chiropractic but occur with most types of spinal manipulation. Incidence figures can, however, not be derived from the data available to date.

The aim of this study ${ }^{391}$ was to summarise all cases in which chiropractic spinal manipulation was followed by death. This study is a systematic review of case reports. Literature searches in four electronic databases with no restrictions of time or language. Twenty six fatalities were published in the medical literature and many more might have remained unpublished. The alleged pathology usually was a vascular accident involving the dissection of a vertebral artery. In conclusion, numerous deaths have occurred after chiropractic manipulations. The risks of this treatment by far outweigh its benefit

\subsection{Massage (1 systematic review)}

After many years out of the limelight, massage therapy is now experiencing a revival. The aim of this systematic review is to evaluate its potential for harm. ${ }^{392}$ Computerised literature searches were carried out in four databases. All articles reporting adverse effects of any type of massage therapy were retrieved. Adverse effects relating to massage oil or ice were excluded. No language restrictions were applied. Data were extracted and evaluated according to predefined criteria. Sixteen case reports of adverse effects and four case series were found. The majority of adverse effects were associated with exotic types of manual massage or massage delivered by laymen, while massage therapists were rarely implicated. The reported adverse events include cerebrovascular accidents, displacement of a ureteral stent, embolisation of a kidney, haematoma, leg ulcers, nerve damage, posterior interosseous syndrome, pseudoaneurism, pulmonary embolism, ruptured uterus, strangulation of neck, thyrotoxicosis and various pain syndromes. In the majority of these instances, there can be little doubt about a cause-effect relationship. Serious adverse effects were associated 
mostly with massage techniques other than 'Swedish' massage. It was concluded that massage is not entirely risk free. However, serious adverse events are probably true rarities.

\subsection{Moxibustion}

The aim of this review ${ }^{393}$ was to identify adverse events of moxibustion as reported in the medical literature. Computerised literature searches were carried out in 14 databases. All articles reporting adverse effects of any type from moxibustion in humans were included, regardless of study design and publication language. The related journals and references in all located articles were manually searched for further relevant articles. Data were extracted and evaluated according to predefined criteria by three independent reviewers. Adverse events related to moxibustion treatment were reported in 4 randomised clinical trials, 1 controlled clinical trial, 2 uncontrolled observational studies, 13 case reports, and 1 prospective study. The most common effects identified in this review were allergic reactions, burns, and infections such as cellulitis and hepatitis C. Allergic reactions were reported in six case reports (four case reports related to infections and two related to burns). The other articles were case reports of xerophthalmia, xeroderma, hyperpigmented macules, ptosis and eversion of the eyelids. In clinical trials, various adverse events such as rubefaction, blistering, itching sensations, discomfort due to smoke, general fatigue, stomach upsets, flare-ups, headaches, and burns were reported. Tenderness and pressure in the epigastric region or in one of the hypochondriac regions, unpleasant odour with or without nausea and throat problems, abdominal pain, premature birth, premature rupture of the membrane and bleeding due to excess pressure on the anterior placenta were reported in pregnant women. Moxibustion is not entirely risk free, as it has several kinds of potential adverse events such as allergy, burn and infection. Currently, the incidence of such events is not known. In the interest of patient safety, sufficiently large prospective studies should be considered to clarify this issue

\subsection{Safety of therapists (6 surveys/6 systematic reviews/1 editorial)}

\subsubsection{Medical herbalists/pregnancy}

The aim of this study was to investigate Internet advice offered by 'medical herbalists' to a pregnant woman regarding herbal treatment of morning sickness. ${ }^{394}$ Search engines were used to find relevant Web sites and all potential e-mail addresses were contacted. Herbalists were asked for advice regarding three specific medicinal herbs: ginger, raspberry and juniper. Eighty-three e-mail addresses were found and contacted. The response rate was $51 \%$. Nineteen (45\%) of all respondents recommended ginger, $9(21 \%)$ of them without mentioning adverse effects. Seven $(17 \%)$ respondents recommended taking raspberry; five (12\%) without mentioning adverse effects. No respondent recommended taking juniper during pregnancy and 12 herbalists (29\%) warned about using this herbal remedy during pregnancy. We concluded that advice about herbal medicine is readily available over the Internet. The advice offered is misleading at best and dangerous at worst. Potential Internet users should be made aware of these problems and ways of minimising the risk should be found.

\subsubsection{Acupuncturists/pacemaker}

Acupuncture is often used for smoking cessation yet it has been shown to be no more effective than sham and to be not entirely free of risk. With more and more patients turning to the Internet for medical advice it seemed appropriate to determine the reliability of the advice given by acupuncturists over the Internet. E-mail addresses were located through a pre-defined strategy and 
randomly divided into three groups. ${ }^{395}$ A letter was sent to groups A and B from a fictitious pacemaker patient asking about the safety and efficacy of electro-acupuncture treatment for smoking cessation. The difference between group A and B was that the patient in group B claimed to live in the acupuncturist's area. Group $\mathrm{C}$ was asked for their advice on electro-acupuncture for smoking cessation as part of a research project. The response rates in group A and B were similar $(55 \%$ group A, 53\% group B) whereas in group C it was significantly lower (30\%). Concerns with electroacupuncture administered to a patient with a pacemaker were issued by $33 \%$ of all acupuncturists. There are, however, significant differences between sub-groups. When the question was asked in the context of a research project, the response was markedly more cautious. Recommendations issued by acupuncturists regarding smoking cessation are not based on the current evidence as to the efficacy of this intervention and concerns about the potential harm of acupuncturists' advice emerge.

\subsubsection{Chiropractors, homoeopaths/vaccination}

Some providers of CAM lend support to the 'anti-vaccination movement' ${ }^{396}$ In particular, sections of the chiropractors, the (non-medically trained) homoeopaths and naturopaths tend to advise their clients against immunisation. The reasons for this attitude are complex and lie, at least in part, in the early philosophies which form the basis of these professions. The negative attitude of some CAM providers towards immunisation constitutes an important example of indirect risks associated with this form of healthcare. The best way forward, it seems, would be a campaign to clarify the riskbenefit profile of immunisations for both users and providers of CAM. An update of the evidence regarding vaccination was recently published. ${ }^{397}$

A survey shows that some homoeopaths and chiropractors advise against MMR. ${ }^{398} \mathrm{We}$ obtained the email addresses of three health professions from these websites: www.homeopath.co.uk/directory, www.chiro-online.com/interadcom, www.internetgp.com/gpsites/alphabet.htm. We also visited the private homepages of homoeopaths and chiropractors on the internet. We sent a letter in which a mother asked for advice about the MMR vaccination for her 1 year old child to all the addresses. We explained to all those who responded that the query was, in fact, part of a research project, giving them opportunity to withdraw their answers. The study was approved by the local ethics committee. We contacted 168 homoeopaths, of whom $104(72 \%)$ responded, 27 (26\%) withdrawing their answers. We contacted 63 chiropractors, of whom 22 (44\%) responded, six (27\%) withdrawing their responses. No general practitioners responded. The table shows that only a few professional homoeopaths and a quarter of the chiropractors advised in favour of the MMR vaccination. Almost half of the homoeopaths and nearly a fifth of the chiropractors advised against it. These data suggest that some providers of complementary medicine are advising people against government policy. General practitioners, on the other hand, seem not to respond at all to patients' emails on this delicate matter.

\subsubsection{Chiropractors}

Chiropractors often use spinal manipulation (SM) to correct such malalignments and treat asthma. Several clinical trials of chiropractic SM exist, but the most rigorous ones are clearly negative. Chronic medication with corticosteroids can lead to osteoporosis, a condition which is a contraindication to chiropractic SM. Given this background, we aimed to determine whether chiropractors would advise an asthma patient on long-term corticosteroids ( 5 years) to try chiropractic as a treatment for this condition. ${ }^{399}$ All 350 e-mail addresses listed at www.interadcom.-com/chiro/html were randomised into two groups. A (deceptive) letter from a (fictitious) patient was sent to group A while group B was asked for advice on chiropractic treatment for asthma as part of a research project. Thus, groups A and B were asked the same question in different contexts: is chiropractic 
safe and effective for an asthma patient on long-term steroids. After data collection, respondents from group A were informed that the e-mail had been part of a research project. Of 97 e-mails in group A, we received 31 responses (response rate $=32 \%$ (95\% CI, 0.23-0.42)). Seventy-four percent (23 respondents) recommended visiting a chiropractor $(95 \% \mathrm{CI}, 0.59-0.89)$. Thirty-five percent (11 respondents) mentioned minimal or no adverse effects of SM (95\% CI, 0.18-0.52). Three chiropractors responded that some adverse effects exist, e.g. risk of bone fracture, stroke. Two respondents noted that other investigations (X-rays, spinal and neurological examination) were required before chiropractic treatment. Three respondents suggested additional treatments and one warned about a possible connection between asthma and measles vaccine. Of 77 e-mails sent to group $\mathrm{B}$, we received 16 responses) response rate $=21 \%(95 \% \mathrm{CI}, 0.17-0.25))$. Eleven respondents $(69 \%)$ recommended visiting a chiropractor $(95 \% \mathrm{CI}, 0.46-0.91)$. Ten respondents mentioned minimal or no adverse effects of SM (95\% CI, 0.39-0.87). Five chiropractors responded that adverse effects of SM exist (e.g. bone fracture). Five respondents suggested pre-testing of the patient to check bone density, allergy, diet, exercise level, hydration and blood. Additional treatments were recommended by three respondents. The pooled results of groups A and B suggested that the majority of chiropractors recommend chiropractic treatment for asthma and the minority mention any adverse effects.

Some chiropractors and their associations claim that chiropractic is effective for conditions that lack sound supporting evidence or scientific rationale. This study ${ }^{400}$ therefore sought to determine the frequency of World Wide Web claims of chiropractors and their associations to treat, asthma, headache/migraine, infant colic, colic, ear infection/earache/otitis media, neck pain, whiplash (not supported by sound evidence), and lower back pain (supported by some evidence). A review of 200 chiropractor websites and 9 chiropractic associations' World Wide Web claims in Australia, Canada, New Zealand, the United Kingdom, and the United States was conducted between 1 October 2008 and 26 November 2008. The outcome measure was claims (either direct or indirect) regarding the eight reviewed conditions, made in the context of chiropractic treatment. We found evidence that 190 (95\%) chiropractor websites made unsubstantiated claims regarding at least one of the conditions. When colic and infant colic data were collapsed into one heading, there was evidence that $76(38 \%)$ chiropractor websites made unsubstantiated claims about all the conditions not supported by sound evidence. Fifty-six (28\%) websites and 4 of the $9(44 \%)$ associations made claims about lower back pain, whereas 179 (90\%) websites and all 9 associations made unsubstantiated claims about headache/migraine. Unsubstantiated claims were made about asthma, ear infection/earache/otitis media, neck pain, The majority of chiropractors and their associations in the English-speaking world seem to make therapeutic claims that are not supported by sound evidence, whilst only $28 \%$ of chiropractor websites promote lower back pain, which is supported by some evidence. We suggest the ubiquity of the unsubstantiated claims constitutes an ethical and public health issue

\subsubsection{Homeopaths}

The websites of all professional homeopathic associations were visited (date 5 August 2008). ${ }^{401}$ Those using languages other than English, French, or German were excluded. All sites were searched for statements on the effectiveness of homeopathy for specific medical conditions. Subsequently, this information was extracted into a table Thirty associations' websites were located of which eight were excluded because of the language restrictions mentioned above. Of the remaining 22 sites, 12 published statements about the effectiveness of homeopathy for specific medical conditions. These data show that about half of all homeopathic associations recommend homeopathic treatments for a wide range of conditions some of which are serious, even lifethreatening. There seems to be little agreement between these recommendations. 
A review of the website of the UK 'Society of Homeopaths' disclosed severe violations of this organisation's own ethical code. ${ }^{402}$

\subsubsection{Reflexologists}

The objective of this survey was to generate preliminary data on how individual reflexologists deal with patients seeking medical advice on the Internet. ${ }^{403}$ An e-mail survey was conducted involving reflexologists who were partly blinded for their advice on the Internet. Two hundred and seventyseven members of the Association of Reflexologists participated. Of 842 e-mails sent out we received 323 responses (38\% response rate) of which 46 participants later withdrew their responses (14\% withdrawal rate). Participants were asked to advise a fictitious patient via e-mail who presented various health problems. Eighty-five percent of all respondents advised the fictitious patient to present the health problems to a medical professional. Fifty-eight percent expressed urgency to see a primary care physician or other health care professional and $95 \%$ pointed out that a diagnosis cannot and should not be made by a reflexologist. Twenty-nine percent of responders suggested a differential diagnosis or underlying causes for the patient's condition. In this survey reflexologists from the UK Association of Reflexologists have responded in an encouraging manner to a fictitious patient's request for health advice via electronic mail as only 5\% (or possibly $15 \%$ ) of reflexologists from this survey need to be more cautious about the advice they give their patients. We hope that our study will further encourage therapists to be more cautious about giving Internet health advice in the future.

\subsection{Websites (4 surveys)}

\subsubsection{Diabetes}

Using a similar approach, we wanted to investigate how good the advice is offered on CM via websites. ${ }^{404}$ For identifying websites most likely to be visited by diabetic patients, the following strategy was adopted. After reviewing the literature about popular search engines, eight were visited on the Internet (on 5 December 2001) to search for the following terms: 'complementary medicine', or 'alternative medicine' or 'complementary therapy' or 'alternative therapy' and 'diabetes'. The first 30 hits through each search engine were recorded. To be included in our study, a website had to be listed on at least three search engines. The sites thus identified were evaluated according to the following criteria: advice regarding conventional therapies, number and nature of complementary/alternative treatments recommended, advice on diabetes prevention, and a judgement whether following advice provided might harm a diabetic patient. We also rated each site according to the scoring system adopted by Sandvik. Our search strategy identified 13 websites. One overtly discouraged diabetic patients from employing conventional anti-diabetic therapies. A large number (total $=372$ ) of CAM treatments were recommended on most websites. Most recommended treatments were of botanical origin. There was an impressive lack of consensus: most treatments are recommended by just one website. The highest degree of consensus (10 recommendations) existed for chromium. Five websites did not mention prevention of diabetes. Our overall rating of the websites ranged from 5 to 12, indicating a wide range of quality. In our judgement, the advice of one website could harm diabetic patients, while a further five were deemed to be potentially harmful.

\subsubsection{Cancer}

A similar study showed equivalent results for cancer. ${ }^{405}$ Most sites recommend therapies for which efficacy has not been demonstrated and some discourage patients from using conventional therapies. 
Many cancer patients search the World Wide Web for information on complementary and alternative medicine. The aim of this study ${ }^{406}$ was to evaluate the quality of such information and identify the treatments most frequently discussed. We used a pre-tested search strategy to identify the websites that are most likely to be used by cancer patients and assessed them according to predefined criteria. Thirty-two websites were included in this analysis. The quality of these sites was scored on a point system and varied between 8 and 14 points with a maximum of 14 . Most sites issued recommendations for a plethora of treatments, which are typically not supported by sound scientific evidence. Three sites had the potential for harming patients through the advice issued. The most frequently discussed therapies were herbal medicines, diets and mind-body therapies. The most popular websites on complementary and alternative medicine for cancer offer information of extremely variable quality. Many endorse unproven therapies and some are outright dangerous.

\subsubsection{Glaucoma}

Our objective was to identify the most popular forms of complementary and alternative medicine (CAM) for glaucoma on the Internet and to assess the quality of the most popular Web sites addressing CAM for glaucoma. ${ }^{407}$ Using a predefined search algorithm, Web sites most likely to receive hits from people searching the Internet for information on CAM therapies for glaucoma were identified. Web sites were evaluated for quality based on the Sandvik score. Each Web site was also assessed for external validation based on listing on the Health on the Net (HON) Foundation Web site in accordance with their code of approval. Finally, the risk each Web site potentially posed to patients was assessed using a previously described scoring method. Twenty-one Web sites were included in our study. Forty-four different CAM therapies were encountered; vitamin $\mathrm{C}$ supplements, forskolin, and diet and lifestyle modulation were the most common CAMs discussed. The mean Sandvik score for all Web sites was 9.4 (standard deviation, 2.6). Only one Web site was listed in the HON Foundation code of approval. Two of the 21 Web sites received the highest risk scores of 3 of a potential 4 points. None of the Web sites discouraged adhering to a clinician's advice. Fourteen of the 21 Web sites assessed provided commercial details promoting CAM, with the possibility of purchasing remedies or arranging consultations. Thirteen Web sites provided opinions and experiences without factual details. Medical information conveyed over the Internet is of variable quality and is not subject to regulation. Many sources of information contain advice on therapies that have no evidence base and may put patients at risk. It is important that researchers and health care providers be aware of the content and quality of this information so as to offer advice to patients about the evidence and potential risks of CAM therapies. This can only be achieved by increasing awareness among ophthalmologists of the range of products and quality of information available for CAM therapies for glaucoma.

\section{Costs of complementary therapies (5 systematic reviews)}

The above-mentioned survey ${ }^{1}$ extrapolated that the integration of CM would amount to very considerable cost to the NHS. However, reliable data are sparse.

The aim of this analysis was to systematically review all reports of economic analysis of complementary and alternative medicine. ${ }^{408}$ Three searches were performed for reports of cost description, cost comparison, cost effectiveness, or cost benefit studies. Prospective studies that investigated comparative groups were considered to be of higher quality. A total of 34 reports were included. Retrospective studies in which a range of therapies are provided in primary care suggest that these may reduce referral and treatment costs, but prospective studies suggest that 
complementary medicine is an additional expense and does not substitute for orthodox care. For individual therapies, one thorough but retrospective study suggests that carefully targeted acupuncture may reduce referral costs for musculoskeletal problems. One large pragmatic study of spinal manipulative therapy suggests that this treatment may reduce the societal costs of back pain, but four controlled trials found that manipulative therapy does not reduce the costs incurred by the back pain patients themselves or by the health insurance provider. Spinal manipulative therapy for back pain may offer cost saving to society, but it does not save money for the purchaser. There is a paucity of rigorous studies that could provide conclusive evidence of differences in costs and outcomes between other complementary therapies and orthodox medicine. The evidence from methodologically flawed studies is contradicted by more rigorous studies, and there is a need for high quality investigations of the costs and benefits of complementary medicine.

A further review of this area made the following key points:

- complementary and alternative therapies reflect a diverse range of interventions

- the emphasis is on illness - what the patient experiences, rather than disease - and what the health professional recognises

- evidence of effectiveness is scarce and there is no valid evidence of cost effectiveness

- there are a number of difficulties undertaking research in this area to inform purchasing decisions

- complementary and alternative medicine cannot be excluded from rigorous evaluation, but may open a broader agenda about the meaning of health. ${ }^{409}$

Another systematic review focussed on all rigorous cost-effectiveness studies conducted in the context of the UK healthcare system. ${ }^{410}$ Only 5 studies met our inclusion criteria. One was on acupuncture, the rest on spinal manipulation. These treatments represented additional healthcare costs in 4 of the 5 studies. No compelling evidence was found to support the notion that CAM would save money. This review was later updated but the conclusions remained essentially the same. $^{411}$

The aim of a further review is to systematically summarise and assess all globally available economic evaluations of complementary and alternative therapies. ${ }^{412}$ Systematic literature searches were performed in seven electronic databases and the reference lists located were checked for further relevant publications. All articles in which original, prospectively collected data from any form of standard economic analysis of complementary and alternative therapies administered for any medical condition to groups of patients was presented were included. No language restrictions were imposed. Study selection, data extraction and quality assessment were performed independently with any disagreements being settled with discussion. Twenty-eight articles were identified relating to the economic evaluation of manipulative therapy, relaxation, acupuncture, hypnosis, plant-based preparations, traditional Chinese medicine, homeopathy and neuroreflexotherapy. Cost effectiveness analyses were included in 27 of the articles; two of which also incorporated a cost-utility analysis. One paper described a cost minimisation analysis. Studies were of medium to good quality with scores ranging from 0.63 to 1.0. Preliminary evidence exists to suggest that some therapies may be superior to the comparative treatment, in terms of costeffectiveness, in the setting of the clinical trials discussed. The addition of some complementary therapies to standard care may also be associated with additional cost with no further clinical benefit. We concluded that there is a paucity of data from rigorous economic evaluations of complementary and alternative therapies. Rigorous economic evaluation of complementary 
therapies with demonstrated effectiveness is needed before conclusions as to the relative cost effectiveness can be drawn.

\section{Summary}

$\mathrm{CM}$ is an immensely diverse and colourful area. ${ }^{413}$ Our dual research strategy is, on the one hand designed to take account of this diversity, and on the other it focuses on the most popular modalities in the UK. This is perhaps nowhere expressed more clearly than in our book ${ }^{414}$ which recently went into its second edition ${ }^{415}$ and was translated into German, French and Korean. We believe it is the most rigorously evidence-based account of CAM to date.

Our work during the last 12 years has been successful and rewarding in several ways:

- we have published around 1000 papers in the peer reviewed medical literature. Our unit's contribution to the CM literature is larger than that of any other unit worldwide.

- we have established active collaboration with more than 20 research groups both in the UK and abroad

- we have identified several areas where CM is of proven effectiveness

- we have identified other areas where the evidence is incomplete and more research needs doing

- we have demonstrated the relative safety of some complementary therapies (e.g. acupuncture, specific herbal medicines)

- we have highlighted some areas where safety concerns exist and more research is required (e.g. herb-drug interactions).

Our hope and strategy for the future is to help complete the evidence-base for CM with a view of maximising the benefit and minimising the risk of CM for the patient.

Professor E Ernst

Chair of Complementary Medicine

Peninsula Medical School

Universities of Exeter \& Plymouth

January 2010

Anyone requiring full reprints of selected articles should write to Complementary Medicine, Peninsula Medical School, Universities of Exeter \& Plymouth, 25 Victoria Park Road, Exeter, EX2 4NT, UK. E-mail: Edzard.Ernst@pms.ac.uk. 


\section{References}

(1) Ernst E, White AR. The BBC survey of complementary medicine use in the UK. Complement Ther Med 2000; 8:32-36.

(2) White AR, Resch KL, Ernst E. Complementary medicine: use and attitudes among GPs. Fam Pract 1997; 14:302-306.

(3) White AR, Resch KL, Ernst E. A survey of complementary practitioners' fees, practice, and attitudes to working within the National Health Service. Comp Ther Med 1997; 5:210214.

(4) Hunt KJ, Coelho HF, Wider B, Perry R, Hung SK, Terry R et al. Complementary and alternative medicine use in England: results from a national survey. Int J Clin Pract 2010; 64(11):1496-1502.

(5) Canter PH, Ernst E. Herbal supplement use by persons aged over 50 years in Britain. Drugs Aging 2004; 21:597-605.

(6) Ernst E. Complementary therapies for asthma: what patients use. J Asthma 1998; 35:667671.

(7) Ernst E. Use of complementary therapies in childhood asthma. Pediatr Allergy Immunol 1998; 12:29-32.

(8) Resch K, Hill S, Ernst E. Use of complementary therapies by individuals with 'arthritis'. Clin Rheumatol 1997; 16:391-395.

(9) Ernst E. The usage of complementary therapies by dermatological patients: a systematic review. Br J Dermatol 2000; 142:857-861.

(10) Ernst E. Complementary AIDS therapies: the good, the bad and the ugly. Int J STD \& AIDS 1997; 8:281-285.

(11) Ernst E. Prevalence of complementary/alternative medicine for children: a systematic review. Eur J Pediatr 1999; 158:7-11.

(12) Ernst E, Cassileth BR. The prevalence of complementary/alternative medicine in cancer: a systematic review. Cancer 1998; 83:777-782.

(13) Ernst E. Usage of complementary therapies in rheumatology. A systematic review. Clin Rheumatol 1998; 17:301-305.

(14) Hunt KJ, Ernst E. Patient's use of CAM: results from the Health Survey for Englnd 2005. FACT 2010; 15(2):101-103.

(15) Wider B, Ernst E. CAM research funding in the UK: surveys of medical charities in 1999 and 2002. Complement Ther Med 2003; 11:165-167.

(16) Ernst E. How much of CAM is based on research evidence? eCAM 2009; May 21. [Epub ahead of print]. 
(17) Lee MS, Lee E-N, Ernst E. Is tai chi beneficial for improving aerobic capacity? a systematic review. Br J Sports Med 2008; 43:569-573.

(18) Ernst E. Complementary therapies for addictions: not an alternative. Addiction 2003; 97:1491-1492.

(19) Ernst E, Lee MS, Choi TY. Acupuncture for addictions: a systematic review of systematic reviews. FACT 2010; 15(2):97-100.

(20) Pittler MH, Verster JC, Ernst E. Interventions for preventing or treating alcohol hangover: systematic review of randomised controlled trials. BMJ 2005; 331:1515-1518.

(21) Pittler MH, Ernst E. Kava for treating anxiety - a meta-analysis of randomized trials. Perfusion 2002; 15:474-481.

(22) Kanji N, White A, Ernst E. Autogenic training to reduce anxiety in nursing students: randomized controlled trial. J Adv Nurs 2006; 53:729-735.

(23) Ernst E. Herbal remedies for anxiety - a systematic review of controlled clinical trials. Phytomedicine 2006; 13:205-208.

(24) Coelho HF, Canter PH, Ernst E. The effectiveness of hypnosis for the treatment of anxiety: a systematic review. Primary Care Community Psychiatry 2007; 12(2):49-63.

(25) Cobb CS, Ernst E. Systematic review of a marine nutriceutical supplement in clinical trials for arthritis: the effectiveness of the New Zealand green-lipped mussel Perna canaliculus. Clin Rheumatol 2006; 25:275-284.

(26) Canter PH, Wider B, Ernst E. The antioxidant vitamins A, C, E and selenium in the treatment of arthritis: a systematic review of randomised clinical trials. Rheumatology 2007; 46(8):1223-1233.

(27) Martin J, Donaldson AN, Villarroel R, Parmar MK, Ernst E, Higginson IJ. Efficacy of acupuncture in asthma: systematic review and meta-analysis of published data from 11 randomised controlled trials. Eur Respir J 2002; 20:846-852.

(28) Ernst E. Spinal manipulation for asthma: a systematic review of randomised clinical trials. Respiratory Med 2009; 103(12):1791-1795.

(29) Huntley A, Ernst E. Herbal medicines for asthma: a systematic review. Thorax 2000; 55:925-929.

(30) Ernst E. Breathing techniques - adjunctive treatment modalities for asthma? A systematic review. Eur Respir J 2000; 15:969-972.

(31) Cooper S, Oborne J, Newton S, Harrison V, Thompson Coon J, Lewis S et al. Effect of two breathing exercises (Buteyko and pranayama) in asthma: a randomised controlled trial. Thorax 2003; 58:674-679.

(32) Huntley A, White AR, Ernst E. Relaxation therapies for asthma: a systematic review. Thorax 2002; 57:127-131. 
(33) White A, Slade P, Hunt C, Hart A, Ernst E. Individualised homeopathy as an adjunct in the treatment of childhood asthma: a randomised placebo controlled trial. Thorax 2003; 58:317-321.

(34) Lee MS, Pittler MH, Shin BC, Kim JI, Ernst E. Acupuncture for allergic rhinitis: a systematic review. Annals of Allergy, Asthma, \& Immunology 2009; 102(4):269-279.

(35) Lee MS, Kim JI, Ernst E. Massage therapy for children with autism spectrum disorders: a systematic review. J Clin Psychiatry 2010;Dec 28. [Epub ahead of print].

(36) Lee H, Schmidt K, Ernst E. Acupuncture for the relief of cancer-related pain - a systematic review. Eur J Pain 2005; 9(4):437-444.

(37) Lee MS, Kim KH, Choi S-M, Ernst E. Acupuncture for hot flashes in breast cancer patients: a systematic review. Breast Cancer Res Treat 2008; Nov 4. [Epub ahead of print].

(38) Lee MS, Kim KH, Shin BC, Choi SM, Ernst E. Acupuncture for treating hot flushes in men with prostate cancer: a systematic review. Supportive Care in Cancer 2009; 17(7):763-770.

(39) Ernst E, Lee MS. Acupuncture for palliative and supportive cancer care: a systematic review of systematic reviews. J Pain Symptom Manage 2010; 40(1):e3-e5.

(40) Wilcock A, Manderson C, Weller R, Walker G, Carr D, Carey A et al. Does aromatherapy massage benefit patients with cancer attending a specialist palliative care day centre? Palliative Med 2004; 18:287-290.

(41) Ernst E. Carctol: Profit before Patients? Breast Care 2009; 4:31-33.

(42) Roffe L, Schmidt K, Ernst E. Efficacy of coenzyme Q10 for improved tolerability of cancer treatments: a systematic review. J Clin Oncol 2004; 22:4418-4424.

(43) Roffe L, Schmidt K, Ernst E. A systematic review of guided imagery as an adjuvant cancer therapy. Psychooncol 2005; 14:607-617.

(44) Boehm K, Borrelli F, Ernst E, Habacher G, Hung SK, Milazzo S et al. Green tea (Camellia sinensis) for the prevention of cancer. Cochrane Database of Syst Rev 2009; Issue 3. Arti No: CD005004.

(45) Milazzo S, Russell N, Ernst E. Efficacy of homeopathic therapy in cancer treatment. Eur J Cancer 2006; 42:282-289.

(46) Ernst E. Massage therapy for cancer palliation and supportive care: a systematic review of randomised clinical trials. Support Care Cancer 2009; 17(4):333-337.

(47) Lee MS, Choi TY, Park JE, Lee SS, Ernst E. Moxibustion for cancer care: a systematic review and meta-analysis. BMC Cancer 2010; 10:130.

(48) Ernst E. The role of complementary and alternative medicine in cancer. Lancet Oncology 2000; 1:176-180.

(49) Ernst E, Cassileth BR. How useful are unconventional cancer treatments? Eur J Cancer 1999; 35:1608-1613. 
(50) Ernst E. A primer of complementary and alternative medicine commonly used by cancer patients. Med J Austr 2001; 174:88-92.

(51) Ernst E. Complementary therapies in palliative cancer care. Cancer 2001; 91:2181-2185.

(52) Ernst E. Can allium vegetables prevent cancer? Phytomed 1997; 4:79-83.

(53) Lee MS, Pittler MH, Ernst E. Is Tai Chi an effective adjunct in cancer care? A systematic review of controlled clinical trials. Support Care Cancer 2007; 15:597-601.

(54) Lee MS, Choi TY, Ernst E. Tai chi for breast cancer patients: a systematic review. Breast Cancer Res Treat 2010; 120(2):309-316.

(55) Ernst E. Thymus therapy for cancer? A criteria-based, systematic review. Eur J Cancer 1997; 33:531-534.

(56) Lee MS, Chen KW, Sancier KM, Ernst E. Qigong for cancer treatment: A systematic review of controlled clinical trials. Acta Oncol 2007; 46:717-722.

(57) Ernst E. Mistletoe for cancer? Eur J Cancer 2001; 37:9-11.

(58) Ernst E, Schmidt K, Steuer-Vogt MK. Mistletoe for cancer? A systematic review of randomised controlled trials. Int J Cancer 2003; 107:262-267.

(59) Milazzo S, Ernst E, Lejeune S, Schmidt K. Laetrile treatment for cancer. The Cochrane Database of Systematic Reviews 2006; Issue 2. Art. No.: CD005476.

(60) Milazzo S, Lejeune S, Ernst E. Laetrile for cancer: a systematic review of the clinical evidence. 15 ed. 2007.

(61) Baum M, Cassileth BR, Daniel R, Ernst E, Filshie J, Nagel GA et al. The role of complementary and alternative medicine in the management of early breast cancer: Recommendations of the European Society of Mastology (EUSOMA). Eur J Cancer 2006; 42(12):1711-1714.

(62) Kim JI, Lee MS, Kang JW, Choi do Y, Ernst E. Reflexology for the symptomatic treatment of breast cancer: a systematic review. Integr Cancer Ther 2010; 9(4):326-330.

(63) Ernst E, Schmidt K, Baum M. Complementary/Alternative therapies for the treatment of breast Cancer. A systematic review of randomized clinical trials and a critique of current terminology. 12 ed. 2006.

(64) Sim H, Shin BC, Lee MS, Jung A, Lee H, Ernst E. Acupuncture for Carpal Tunnel Syndrome: A Systematic Review of Randomized Controlled Trials. J Pain 2010; Nov 17. [Epub ahead of print].

(65) Guo R, Pittler MH, Ernst E. Herbal medicines for the treatment of COPD: a systematic review. Eur Respir J 2006; 28(5):330-338.

(66) Pittler MH, Schmidt K, Ernst E. Hawthorn extract for treating chronic heart failure: metaanalysis of randomized trials. Am J Med 2003; 114:665-674.

(67) Pittler MH, Guo R, Ernst E. Hawthorn extract for treating chronic heart failure. Cochrane Database Syst Rev 2008; 1 CD005312. 
(68) Canter PH, Ernst E. Multiple $\mathrm{n}=1$ trials in the identification of responders and nonresponders to the cognitive effects of Ginkgo biloba. Int J Clin PharmacolTher 2003; 41:354-357.

(69) Canter PH, Ernst E. Ginkgo biloba: a smart drug? A systematic review of controlled trials of the cognitive effects of Ginkgo biloba extracts in healthy people. Psychopharmacol Bull 2002; 36:108-123.

(70) Canter PH, Ernst E. Ginkgo biloba is not a smart drug: an updated systematic review of randomised clinical trials testing the nootropic effects of $G$. biloba extracts in healthy people. Hum Psychopharmacol 2007; 22:265-278.

(71) Canter PH, Ernst E. The cumulative effects of Transcendental Meditation on cognitive function - a systematic review of randomised controlled trials. Wien Klin Wochenschr $2003 ; 115: 758-766$.

(72) Ernst E. Abdominal massage therapy for chronic constipation: a systematic review of controlled clinical trials. Forsch Komplementärmed 1999; 6:149-151.

(73) Lee MS, Choi TY, Park JE, Ernst E. Effects of moxibustion for constipation treatment: a systematic review of randomized controlled trials. Chin Med 2010; 5:28.

(74) Lee S, Lee MS, Choi JY, Lee SW, Jeong SY, Ernst E. Acupuncture and heart rate variability: a systematic review. Auton Neurosci 2010; 155(1-2):5-13.

(75) Kanji N, White AR, Ernst E. Autogenic training reduces anxiety after coronary angioplasty: a randomised clinical trial. Am Heart J 2004; 147:e10.

(76) Asbury E, Kanji N, Ernst E, Barbir M, Collins P. Autogenic training to manage symptomology in women with chest pain and normal coronary arteries. Menopause 2008; 16(1):60-65.

(77) Ernst E. Chelation therapy for coronary heart disease: An overview of all clinical investigations. Am Heart J 2000; 140:139-141.

(78) Cohen PA, Ernst E. Safety of herbal supplements: a guide for cardiologists. Cardiovasc Ther 2010; 28(4):246-253.

(79) Schmidt K, Ernst E. Music therapy for patients with cardiovascular diseases - a systematic review. Perfusion 2004; 17:136-144.

(80) Lee MS, Pittler MH, Taylor-Piliae RE, Ernst E. Tai chi for cardiovascular disease and its risk factors: a systematic review. J Hypertension 2007; 25:1974-1977.

(81) Ernst E, Barnes J. Are homoeopathic remedies effective for delayed-onset muscle soreness? A systematic review of placebo-controlled trials. Perfusion 1998; 11:4-8.

(82) Ernst E. Does post-exercise massage treatment reduce delayed onset muscle soreness? A systematic review. Br J Sports Med 1998; 32:212-214.

(83) Lee MS, Shin B-C, Ernst E. Acupuncture for Alzheimer's disease: a systematic review. Int J Clin Pract 2009; 63(6):874-879. 
(84) Ernst E. Diet and dementia, is there a link? A systematic review. Nutr Neuroscience 1999; 2:1-6.

(85) Ernst E, Pittler MH. Ginkgo biloba for dementia: a systematic review of double-blind placebo-controlled trials. Clin Drug Invest 1999; 17:301-308.

(86) Lee MS, Yang EJ, Kim JI, Ernst E. Ginseng for cognitive function in Alzheimer's Disease: A systematic review. J Alzheimer's Dis 2009; 18(2):339-344.

(87) Mukaino Y, Park J, White A, Ernst E. The effectiveness of acupuncture for depression: a systematic review of randomised controlled trials. Acupunc Med 2005; 23:70-76.

(88) Ernst E, Lee MS, Choi TY. Acupuncture for Depression? A Systematic Review of Systematic Reviews. Eval Health Prof 2010.

(89) Coelho HF, Boddy K, Ernst E. Massage therapy for the treatment of depression: a systematic review. Int J Clin Pract 2008; 62(2):325-333.

(90) Ernst E, Rand JI, Stevinson C. Complementary therapies for depression. Arch Gen Psychiatry 1998; 55:1026-1032.

(91) Ernst E. St John's wort, an anti-depressant? A systematic, criteria-based review. Phytomed $1995 ; 2: 67-71$.

(92) Stevinson C, Ernst E. Hypericum for depression. An update of the clinical evidence. Eur Neuropsychopharmacol 1999; 9:501-505.

(93) Di Carlo G, Borrelli F, Ernst E, Izzo AA. St John's wort: Prozac from the plant kingdom. Trends Pharmacol Sci 2001; 22:292-297.

(94) Ernst E. Complementary/alternative medicine for diabetic patients? Curr Med Lit Diabetes 2002; 19:53-57.

(95) Ernst E. Plants with hypoglycemic activity in humans. Phytomed 1997; 4:73-78.

(96) Lee MS, Chen KW, Choi Y-Y, Ernst E. Qigong for type 2 diabetes care: A systematic review. Complement Ther Med 2009; 17:236-242.

(97) Lee MS, Pittler MH, Kim MS, Ernst E. Tai chi for Type 2 diabetes: a systematic review. Diabet Med 2008; 25(2):240-241.

(98) Lee MS, Shin BC, Choi TY, Ernst E. Acupuncture for treating dry eye: a systematic review. Acta Ophthalmol 2010;Mar 16. [Epub ahead of print].

(99) Armstrong NC, Ernst E. The treatment of eczema with Chinese herbs. A systematic review of randomized clinical trials. Br J Clin Pharmacol 1999; 48:262-264.

(100) Ernst E, Pittler MH, Stevinson C. Complementary/alternative medicine in dermatology. Am J Clin Dermatol 2002; 3:341-348.

(101) Canter P.H., Brown L, Greaves C, Ernst E. Joheri Family Healing: A pilot study. Evid Based Complement Alternat Med 2006; 3:533-540. 
(102) Pittler MH, Armstrong NC, Cox A, Collier PM, Hart A, Ernst E. Randomized, doubleblind, placebo-controlled trial of autologous blood therapy for atopic dermatitis. $\mathrm{Br} \mathrm{J}$ Dermatol 2003; 148:307-313.

(103) Lee H, Ernst E. Acupuncture for GI endoscopy: a systematic review. Gastrointest Endosc 2005; 60(15):784-789.

(104) Lee MS, Shin BC, Ernst E. Acupuncture for treating erectile dysfunction: a systematic review. BJU International 2009; 104(3):366-370.

(105) Shin B-C, Lee MS, Yang EJ, Lim HS, Ernst E. Maca (L. meyenii) for improving sexual function: a systematic review. BMC Complement Altern Med 2010; 10:44.

(106) Jang D-J, Lee MS, Shin B-C, Lee Y-C, Ernst E. Red ginseng for treating erectile dysfunction: a systematic review. Br J Clin Pharm 2008; 66(4):444-450.

(107) Stevinson C, Dixon M, Ernst E. Hypericum for fatigue - a pilot study. Phytomed 1998; 5:443-447.

(108) Mayhew E, Ernst E. Acupuncture for fibromyalgia - a systematic review of randomized clinical trials. Rheumatology 2006 Dec 19; [Epub ahead of print]. Rheumatology (Oxford) 2008; 46(5):801-804.

(109) Ernst E. Chiropractic treatment for fibromyalgia: a systematic review. Clin Rheumatol 2009; 28(10):1175-1178.

(110) De Silva V, El-Metwally A, Ernst E, Lewith G, Macfarlane GJ. Evidence for the efficacy of complementary and alternative medicine in the management of fibromyalgia: a systematic review. Rheumatol 2010; 49(6):1063-1068.

(111) Perry R, Terry R, Ernst E. A systematic review of homoeopathy for the treatment of fibromyalgia. Clin Rheumatol 2010; 29(5):457-464.

(112) Melchart D, Linde K, Fischer P, White A, Allais G, Vickers A et al. Acupuncture for recurrent headaches: a systematic review of randomized controlled trials. Cephalalgia 1999; 19:779-786.

(113) White AR, Eddleston C, Hardie R, Resch KL, Ernst E. A pilot study of acupuncture for tension headache, using a novel placebo. Acupunct Med 1996; 14:11-15.

(114) White AR, Resch K-L, Chan JCK, Norris CD, Modi SK, Patel JN et al. Acupuncture for episodic tension-type headache: a multicentre randomized controlled trial. Cephalalgia 2000; 20:632-637.

(115) Kanji N, White AR, Ernst E. Autogenic training for tension type headaches: A systematic review of controlled trials. Comp Ther Med 2006; 14:144-150.

(116) Pittler MH, Vogler BK, Ernst E. Feverfew for preventing migraine. The Cochrane Library 2000; 3:1-10.

(117) Ernst E. Homoeopathic prophylaxis of headaches and migraine? A systematic review. J Pain Sympt Managem 1999; 18:353-357. 
(118) Astin JA, Ernst E. The effectiveness of spinal manipulation for the treatment of headache disorders: A systematic review of randomized clinical trials. Cephalalgia 2002; 22:617623.

(119) Mills E, Wu P, Ernst E. Complementary therapies for the treatment of HIV: in search of the evidence. Int J STD AIDS 2005; 16:395-402.

(120) Stevinson C, Pittler MH, Ernst E. Garlic for treating hypercholesterolemia. A metaanalysis of randomized clinical trials. Ann Intern Med 2000; 133:420-429.

(121) Ernst E. Garlic (Allium sativum) a cholesterol-lowering agent? Perfusion 2005; 18:321323.

(122) Wider B, Pittler MH, Thompson-Coon J, Ernst E. Artichoke leaf extract for treating hypercholesterolaemia. Cochrane Database of Syst Rev 2009; Issue 4:Art. No.:

CD003335. DOI: 10.1002/14651858.CD003335.pub2.

(123) Thompson Coon J., Ernst E. Herbs for serum cholesterol reduction: a systematic review. J Fam Pract 2003; 52:468-478.

(124) Ernst E. Complementary/alternative medicine for hypertension: a mini-review. Wien Med Wochenschr 2005; 155:386-391.

(125) Hur MH, Lee MS, Kim C, Ernst E. Aromatherapy for treatment of hypertension: a systematic review. J Eval Clin Pract 2010;Jul 29. [Epub ahead of print].

(126) Kanji N, White AR, Ernst E. Anti-hypertensive effects of autogenic training: A systematic review. Perfusion 1999; 12:279-282.

(127) Ernst E. Chiropractic spinal manipulation as a treatment of hypertension? A systematic review of randomised clinical trials. Perfusion 2008; 21:188-190.

(128) Hur MH, Lee MS, Yang HJ, Kim C, Bae I-L, Ernst E. Ginseng for Reducing the Blood Pressure in Patients with Hypertension: A Systematic Review and Meta-Analysis. J Ginseng Res 2010; 34(4):342-347.

(129) Kim JI, Choi JY, Lee H, Lee MS, Ernst E. Moxibustion for hypertension: a systematic review. BMC Cardiovasc Disord 2010; 10:33.

(130) Lee MS, Pittler MH, Guo R, Ernst E. Qigong for hypertension: a systematic review of randomized clinical trials. J Hypertension 2007; 25:1525-1532.

(131) Lee MS, Lee EN, Kim JI, Ernst E. Tai chi for lowering resting blood pressure in the elderly: a systematic review. J Eval Clin Pract 2010; 16(4):818-824.

(132) Canter PH, Ernst E. Insufficient evidence to conclude whether or not Transcendental Meditation decreases blood pressure: results of a systematic review of randomized clinical trials. J Hypertens 2004; 22:2049-2054.

(133) Ernst E. Chiropractic spinal manipulation for infant colic: a systematic review of randomised clinical trials. Int J Clin Pract 2009; 63(9):1351-1353. 
(134) Guo R, Pittler MH, Ernst E. Complementary medicine for treating or preventing influenza or influenza-like illness. Am J Med 2007; 120:923-929.

(135) Basch E, Ulbricht C, Basch S, Dalton S, Ernst E, Foppa I et al. An evidence-based systemic review Echinacea E. angustifolia DC, E. pallida, E. purpurea by the Natural Standard Research Collaboration. J Herb Pharmacother 2005; 5(2):57-88.

(136) Ernst E, März R, Sieder C. A controlled multi-centre study of herbal versus synthetic secretolytic drugs for acute bronchitis. Phytomed 1997; 4:287-293.

(137) Ernst E. Is garlic an effective treatment for helicobacter pylori infection? Arch Intern Med 1999; 159:2484-2485.

(138) Thompson Coon J., Ernst E. Andrographis paniculata in the treatment of upper respiratory tract infections: a systematic review of safety and efficacy. Planta Med 2004; 70:293-298.

(139) Agbabiaka TB, Guo R, Ernst E. Pelargonium sidoides for acute bronchitis: A systematic review and meta-analysis. Phytomedicine 2008; 15(5):378-385.

(140) Martin KW, Ernst E. Antiviral agents from plants and herbs: a systematic review. Antiviral Therapy 2003; 8:77-90.

(141) Martin KW, Ernst E. Herbal medicines for treatment of bacterial infections: a review of controlled clinical trials. J Antimicrobial Chemother 2003; 51:241-246.

(142) Martin KW, Ernst E. Herbal medicines for treatment of fungal infections: a systematic review of controlled clinical trials. Mycoses 2004; 47:87-92.

(143) Özsoy M, Ernst E. How effective are complementary therapies for HIV and AIDS? - a systematic review. Int J STD \& AIDS 1999; 10:629-635.

(144) Lee MS, Shin B-C, Suen LKP, Park T-Y, Ernst E. Auricular acupuncture for insomnia: a systematic review. Int J Clin Pract 2008; 62(11):1744-1752.

(145) Stevinson C, Ernst E. Valerian for insomnia: a systematic review of randomized clinical trials. Sleep Med 2000; 1:91-99.

(146) Pittler MH, Ernst E. Peppermint oil for irritable bowel syndrome: a critical review and meta-analysis. Am J Gastroenterol 1998; 93:1131-1135.

(147) Ernst E, Pittler MH. Phytotherapie des Colon irritabile: ein systematischer Review. In: Rietbrock N.Phyto-Pharmaka VI, forschung und klinische Anwendung. Darmstadt; Steinkopff Verlag. 2000;229-234.

(148) Lee H, Ernst E. Acupuncture for labor pain management: a systematic review. Am J Obstet Gynecol 2004; 191:1573-1579.

(149) Cho SH, Lee H, Ernst E. Acupuncture for pain relief in labour: a systematic review and meta-analysis. BJOG 2010; 117(8):907-920.

(150) Ernst E, Pittler MH. Experts' opinions on complementary/alternative therapies for low back pain. J Manip Phys Ther 1999; 22:87-90. 
(151) Ernst E, Pittler MH, Wider B, Boddy K. Complementary therapies for back pain: is the evidence getting stronger? Clin Rheumatol 2007; 26:736-738.

(152) Ernst E, White AR. Acupuncture for back pain. A meta-analysis of randomized controlled trials. Arch Intern Med 1998; 158:2235-2241.

(153) Manheimer E, White A, Berman B, Ernst E. Meta-analysis: acupuncture for low back pain. Ann Intern Med 2005; 142:651-663.

(154) Ernst E, Canter PH. Chiropractic spinal manipulation treatment for back pain? A systematic review of randomised clinical trials. Phys Ther Rev 2003; 8:85-91.

(155) Ernst E. Massage therapy for low back pain. A systematic review. J Pain Symptom Manage 1999; 17:65-69.

(156) Ernst E. Massage therapy for low back pain: an update. Alt Ther Women's Health 2001; Sept:69-71.

(157) Ernst E. Massage treatment for back pain. BMJ 2003; 326:562-563.

(158) Pittler MH, Karagulle MZ, Karagulle M, Ernst E. Spa therapy and balneotherapy for treating low back pain: meta-analysis of randomized trials. Rheumatology (Oxford) 2006; 45:880-884.

(159) Borrelli F, Ernst E. Alternative and complementary therapies for the menopause. Maturitas 2010; 66(4):333-343.

(160) Lee MS, Shin BC, Ernst E. Acupuncture for treating menopausal hot flushes: a systematic review. Climacteric 2009; 12(1):16-25.

(161) Ernst E, Stevinson C. Herbal medicines and phyto-oestrogens as treatments for menopausal symptoms. Medicine 2001; 29(10):19-22.

(162) Clement YN, Onakpoya I, Hung SK, Ernst E. Effects of herbal and dietary supplements on cognition in menopause: A systematic review. Maturitas 2011.

(163) Borrelli F, Ernst E. Cimifuga racemosa: a systematic review of its clinical efficacy. Eur J Clin 2002; 58:235-241.

(164) Borrelli F, Ernst E. Black cohosh (Cimicifuga racemosa) for menopausal symptoms: a systematic review of its efficacy. Pharmacological Res 2008; 58:8-14.

(165) Thompson Coon J, Pittler MH, Ernst E. Trifolium pratense isoflavones in the treatment of menopausal hot flushes: a systematic review and meta-analysis. Phytomedicine 2007; 14:153-159.

(166) Williamson J, White A, Hart A, Ernst E. Randomised controlled trial of reflexology for menopausal symptoms. Br J Obstet Gynaecol 2002; 109:1050-1055.

(167) Huntley A, Ernst E. Complementary and alternative therapies for treating multiple sclerosis symptoms: a systematic review. Complement Ther Med 2000; 8:97-105.

(168) Cummings TM, White AR. Needling therapies in the management of myofascial trigger point pain: a systematic review. Arch Phys Med Rehabil 2001; 82:986-992. 
(169) Borrelli F, Capasso R, Aviello G, Pittler MH, Izzo AA. Effectiveness and safety of ginger in the treatment of pregnancy-induced nausea and vomiting. Obstet Gynecol 2005; 105:849-856.

(170) Ernst E, Pittler MH. Efficacy of ginger for nausea and vomiting: a systematic review of randomized clinical trials. Br J Anaesth 2000; 84:367-371.

(171) Ernst E. Acupressure for nausea: A best evidence analysis. Eur J Phys Med Rehab 1996; 6:28-29.

(172) Knight B, Mudge C, Openshaw S, White AR, Hart A. Effect of acupuncture on nausea of pregnancy: A randomized controlled trial. Obstet Gynecol 2001; 97:184-188.

(173) Ross J, White A, Ernst E. Western minimal acupuncture for neck pain: a cohort study. Acupunct Med 1999; 17:5-8.

(174) White AR, Ernst E. A systematic review of randomized controlled trials of acupuncture for neck pain. Rheumatol 1999; 38:143-147.

(175) Ernst E. Chiropractic spinal manipulation for neck pain - a systematic review. J Pain 2003; 4:417-442.

(176) White AR, Resch KL, Ernst E. Randomized trial of acupuncture for nicotine withdrawal symptoms. Arch Intern Med 1998; 158:2251-2255.

(177) White AR, Rampes H, Ernst E. Acupuncture for smoking cessation. The Cochrane Library 1999; 4:1-10.

(178) Abbot NC, Stead LF, White AR, Barnes J, Ernst E. Hypnotherapy for smoking cessation. In: The Cochrane Library; Issue 2 1998; Oxford: Update Software.

(179) Thompson Coon J, Ernst E. Systematic review: herbal medicinal products for non-ulcer dyspepsia. Aliment Pharmacol Ther 2002; 16:1689-1699.

(180) Ernst E. Complementary or alternative therapies for osteoarthritis. Nature Clinical Practice Rheumatology 2006; 2:74-80.

(181) Harlow T, Greaves C, White A, Brown L, Hart A, Ernst E. Randomised controlled trial of magnetic bracelets for relieving pain in osteoarthritis of the hip and knee. BMJ 2004; 329:1450-1454.

(182) Ernst E. Acupuncture as a symptomatic treatment of osteoarthritis. Scand J Rheumatol 1997; 26:444-447.

(183) Kwon YD, Pittler MH, Ernst E. Acupuncture for peripheral joint osteoarthritis: a systematic review and meta-analysis. Rheumatology (Oxford) 2006; 45(11):1331-1337.

(184) Long L, Ernst E. Homeopathic remedies for the treatment of osteoarthritis a systematic review. Br Homeopathic J 2001; 90:37-43.

(185) Ernst E. Avocado-soybean unsaponifiables (ASU) for osteoarthritis - a systematic review. Clin Rheumatol 2003; 22:285-288. 
(186) De Silva V, El-Metwally A, Ernst E, Lewith G, Macfarlane GJ. Evidence for the efficacy of complementary and alternative medicines in the management of osteoarthritis: a systematic review. Rheumatology 2010;10.1093/rheumatology/keq379.

(187) Lee MS, Pittler MH, Ernst E. Tai chi for osteoarthritis: a systematic review. Clin Rheumatol 2007; 27(2):211-218.

(188) Ernst E. Exercise for female osteoporosis. A systematic review of randomised clinical trials. Sports Med 1998; 25:359-368.

(189) Lee MS, Pittler MH, Shin B-C, Ernst E. Tai chi for osteoporosis: a systematic review. Osteoporosis Int 2008; 19:139-146.

(190) Preisinger E, Alacamlioglu Y, Pils K, Metka M, Schneider B, Ernst E. Regular Physical Exercises Delay Forearm Bone Loss - Results of Five Controlled Trials. Eur J Phys Med Rehab 1995; 5:8-12.

(191) Ernst E. Re: Chiropractic for otitis? Int J Clin Pract 2009; 63(9):1393.

(192) Pittler MH, Ernst E. Complementary therapies for neuropathic and neuralgic pain. Clin J Pain 2008; 24(8):731-733.

(193) Ernst E, Pittler MH. The effectiveness of acupuncture in treating acute dental pain: a systematic review. Br Dent J 1998; 184:443-447.

(194) Lee H, Ernst E. Acupuncture analgesia during surgery: a systematic review. Pain 2005; 114(3):511-517.

(195) Usichenko TI, Lehmann C, Ernst E. Auricular acupuncture for postoperative pain control: a systematic review of clinical trials. Anaesthesia 2008; 63:1343-1348.

(196) Lee MS, Pittler MH, Shin B-C, Kong JC, Ernst E. Bee venom acupuncture for musculoskeletal pain: a systematic review. J Pain 2008; 9(4):289-297.

(197) Ernst E. Chiropractic manipulation for non-spinal pain - a systematic review. NZ Med J 2003; 116:1-9.

(198) Kim J-I, Lee MS, Lee D-H, Boddy K, Ernst E. Cupping for treating pain: a systmatic review. eCAM 2009; In press.

(199) Stevinson C, Devaraj VS, Fountain-Barber A, Hawkins S, Ernst E. Homeopathic arnica for prevention of pain and bruising: a randomized placebo-controlled trial in hand surgery. $\mathrm{J}$ Roy Soc Med 2003; 96:60-65.

(200) Ernst E. The benefits of Arnica: 16 case reports. Homeopathy 2003; 92:217-219.

(201) Fialka V, Korpan M, Saradeth T, Paternostro-Slugo T, Hexel O, Frischenschlager O et al. Autogenic training for reflex sympathetic dystrophy: a pilot study. Complement Ther Med 1996; 4:103-105.

(202) Pittler MH, Brown EM, Ernst E. Static magnets for reducing pain: systematic review and meta-analysis of randomised trials. CMAJ 2007; 177(7):736-742. 
(203) Lee MS, Choi TY, Kang JW, Lee BJ, Ernst E. Moxibustion for treating pain: a systematic review. Am J Chin Med 2010; 38(5):829-839.

(204) Posadzki P, Ernst E. Osteopathy for musculoskeletal pain patients: a systematic review of randomized controlled trials. Clin Rheumatol 2011; 30(2):285-291.

(205) Lee MS, Pittler MH, Ernst E. External Qigong for pain conditions: a systematic review of randomized clinical trials. J Pain 2007; 8(11):827-831.

(206) Lee MS, Pittler MH, Ernst E. Internal qigong for pain conditions: A systematic Review. J Pain 2009; 10(11):1121-1127.

(207) Abbot NC, Harkness EF, Stevinson C, Marshall FP, Conn DA, Ernst E. Spiritual healing as a therapy for chronic pain: a randomized, clinical trial. Pain 2001; 91:79-89.

(208) Ernst E, Filshie J, Hardy J. Evidence-based complementary medicine for palliative cancer care: does it make sense? Palliative Med 2003; 17:704-707.

(209) Lee MS, Shin B-C, Kong JC, Ernst E. Effectiveness of acupuncture for Parkinson's disease: A systematic review. Mov Disord 2008; 23(11):1505-1515.

(210) Ernst E, Brown R. Complementary therapy for Parkinson's? In: Coene EH, Griffiths RK (eds).Parkinson's Disease A self-care manual.Amsterdam; September Foundation. 2000;174-181.

(211) Lee MS, Lam P, Ernst E. Effectiveness of tai chi for Parkinson's disease: A critical review. Parkinsonism Relat Disord 2008; 14(8):589-594.

(212) Hunt K, Ernst E. The evidence-base for complementary medicine in children: a critical overview of systematic reviews. Arch Dis Child 2010;Jul 6. [Epub ahead of print].

(213) Pittler MH, Ernst E. Complementary therapies for peripheral arterial disease: systematic review. Atherosclerosis 2005; 181:1-7.

(214) Pittler MH, Ernst E. Ginkgo biloba extract for the treatment of intermittent claudication. A meta-analysis. Am J Med 2000; 108:276-281.

(215) Leng GC, Fowler B, Ernst E. Exercise for intermittent claudication. In: Fowkes FGR; Janzon L; Kleijnen J; Leng GC (eds). Peripheral Vascular Diseases Module of the Cochrane Database of Systematic Reviews. The Cochrane Collaboration; Issue 1 Oxford: Update Software; 1998; 1:1-13.

(216) Ernst E. Chelation therapy for peripheral arterial occlusive disease. Circulation 1997; 96:1031-1033.

(217) Barnes J, Resch KL, Ernst E. Homeopathy for Postoperative Ileus. J Clin Gastroenterol 1997; 25:628-633.

(218) Stevinson C, Ernst E. Complementary/alternative therapies for premenstrual syndrome: A systematic review of randomized controlled trials. Am J Obstet Gynecol 2001; 185:227235. 
(219) Stevinson C, Ernst E. A pilot study of Hypericum perforatum for the treatment of premenstrual syndrome. Br J Obstet Gynaecol 2000; 107:870-876.

(220) Kim KH, Lee MS, Choi S-M, Ernst E. Acupuncture for treating uremic pruritus in patients with end-stage renal disease: a systematic review. J Pain Symptom Manag 2010; 40(1):117-125.

(221) Gooding SM, Canter PH, Coelho HF, Boddy K, Ernst E. Systematic review of topical capsaicin in the treatment of pruritus. Int J Dermatol 2010; 49(8):858-865.

(222) Ernst E. Complementary and alternative medicine in rheumatology. Baillieres Clin Rheumatol 2000; 14(4):731-749.

(223) Ernst E. Complementary and alternative medicine for pain management in rheumatic disease. Curr Opin Rheumatol 2002; 14:58-62.

(224) Ernst E, Lee MS. Acupuncture for rheumatic conditions: an overview of systematic reviews. Rheumatol 2010; 49(10):1957-1961.

(225) Ernst E, Chrubasik S. Phyto-anti-inflammatories: A systematic review of randomized, placebo-controlled, double-blind trials. Rheum Dis Clin North Am 2000; 26(1):13-27.

(226) Ernst E. The efficacy of Phytodolor ${ }^{\circledR}$ for the treatment of musculoskeletal pain - a systematic review of randomized clinical trials. Natural Med J 1999; 2:14-17.

(227) Lee MS, Shin B-C, Ernst E. Acupuncture for rheumatoid arthritis: a systematic review. Rheumatology 2008; 47(12):1747-1753.

(228) Soeken KL, Miller SA, Ernst E. Herbal medicines for the treatment of rheumatoid arthritis: a systematic review. Rheumatology 2003; 42:652-659.

(229) Park J, Ernst E. Ayurvedic medicine for rheumatoid arthritis: a systematic review. Semin Arthritis Rheum 2005; 34:705-713.

(230) Lee MS, Pittler MH, Ernst E. Tai chi for rheumatoid arthritis: systematic review. Rheumatology 2007; 46(11):1648-1651.

(231) Canter PH, Lee HS, Ernst E. A systematic review of randomised clinical trials of Tripterygium wilfordii for rheumatoid arthritis. Phytomedicine 2006; 13:371-377.

(232) Guo R, Canter PH, Ernst E. Herbal medicines for the treatment of rhinosinusitis: a systematic review. Otolaryngol Head Neck Surg 2006; 135:496-506.

(233) Lee MS, Shin B-C, Ronan P, Ernst E. Acupuncture for schizophrenia: a systematic review and meta-analysis. Int J Clin Pract 2009; 63(11):1622-1633.

(234) Ojay A, Ernst E. Can singing exercises reduce snoring? A pilot study. Complement Ther Med 2000; 8:151-156.

(235) Armstrong NC, Ernst E. A randomised; double-blind; placebo-controlled trial of Bach Flower Remedy. Perfusion 1999; 11:440-446.

(236) Pittler MH, Ernst E. Efficacy of kava extract for treating anxiety: systematic review and meta-analysis. J Clin Psychopharmacol 2000; 20:84-89. 
(237) Kanji N, Ernst E. Autogenic training for stress and anxiety: a systematic review. Complement Ther Med 2000; 8:106-110.

(238) Hills S, Eckett MJH, Paterson C, Harkness EF. A pilot study to evaluate effects of floatation spa treatment on patients with osteoarthritis. Complement Ther Med 1999; $7: 235-238$.

(239) Ernst E, White AR. Acupuncture as an adjuvant therapy in stroke rehabilitation? Wien Med Wochenschr 1996; 146(21/22):556-558.

(240) Park J, Hopwood V, White AR, Ernst E. Effectiveness of acupuncture for stroke: A systematic review. J Neurol 2001; 248:558-563.

(241) Park J, White A, James M, Hemsley A, Johnson P, Chambers P et al. Acupuncture for acute stroke rehabilitation: a sham-controlled, subject-and assessor-blind randomised trial. Arch Intern Med 2005; 165:2026-2031.

(242) Kong JC, Lee MS, Shin BC, Song YS, Ernst E. Acupuncture for functional recovery after stroke: a systematic review of sham-controlled randomized clinical trials. CMAJ 2010; 182(16):1723-1729.

(243) Ernst E, Lee MS, Choi TY. Acupuncture for stroke rehabilitation? An overview of systematic reviews. Perfusion 2010; 23:141-145.

(244) Lee MS, Choi TY, Shin BC, Han CH, Ernst E. Cupping for stroke rehabilitation: a systematic review. J Neurol Sci 2010; 294(1-2):70-73.

(245) Lee MS, Shin BC, Kim JI, Han CH, Ernst E. Moxibustion for stroke rehabilitation: systematic review. Stroke 2010; 41(4):817-820.

(246) Ernst E, White A. Acupuncture as a treatment for temporomandibular joint dysfunction. Arch Otolaryngol Head Neck Surg 1999; 125:269-272.

(247) Park J, White AR, Ernst E. Efficacy of acupuncture as a treatment for tinnitus. Arch Otolaryngol Head Neck Surg 2000; 126:489-492.

(248) Ernst E, Stevinson C. Ginkgo biloba for tinnitus: a review. Clin Otolaryngol 1999; 24:164167.

(249) Lee DH, Kim JI, Lee MS, Choi TY, Choi SM, Ernst E. Moxibustion for ulcerative colitis: a systematic review and meta-analysis. BMC Gastroenterol 2010; 10:36.

(250) Pittler MH, Ernst E. Horse-chestnut seed extract for chronic venous insufficiency: A criteria-based systematic review. Arch Dermatol 1998; 134:1356-1360.

(251) Pittler MH, Ernst E. Horse chestnut seed extract for chronic venous insufficiency. The Cochrane Library 2002; Issue 1:Oxford.

(252) Ernst E. Acupuncture/acupressure for weight reduction? A systematic review. Wien Klin Wochenschr 1997; 109/2:60-62. 
(253) Nourshahi M, Ahmadizad S, Nikbakht H, Heidarnia MA, Ernst E. The effects of triple therapy (acupuncture, diet and exercise) on body weight: a randomized, clinical trial. Int $\mathbf{J}$ Obesity 2009;1-5.

(254) Pittler MH, Ernst E. Complementary therapies for reducing body weight: a systematic review. Int J Obes (Lond) 2005; 29:1030-1038.

(255) Onakpoya IJ, Wider B, Pittler MH, Ernst E. Food supplements for body weight reduction: a systematic review of systematic reviews. Obesity $2011 ; 19(2): 239-244$.

(256) Ernst E, Pittler MH. Chitosan as a treatment for body weight reduction? A meta-analysis. Perfusion 1998; 11:461-465.

(257) Pittler MH, Abbot NC, Harkness EF, Ernst E. Randomized, double-blind trial of chitosan for body weight reduction. Eur J Clin Nutrition 1999; 53:379-381.

(258) Pittler MH, Stevinson C, Ernst E. Chromium picolinate for reducing body weight: metaanalysis of randomized trials. Int J Obesity 2003; 27:522-529.

(259) Onakpoya I, Hung SK, Perry R, Wider B, Ernst E. The Use of Garcinia Extract (Hydroxycitric Acid) as a Weight loss Supplement: A Systematic Review and MetaAnalysis of Randomised Clinical Trials. J Obes 2011; 2011:509038.

(260) Pittler MH, Ernst E. Guar gum for body weight reduction: meta-analysis of randomized trials. Am J Med 2001; 110:724-730.

(261) Ernst E, Hentschel C. Diagnostic methods in complementary medicine. Which craft is witchcraft? Int J Risk Safety Med 1995; 7:55-63.

(262) Ernst E. Komplementärmedizinische Diagnoseverfahren. Deutsches Ärzteblatt 2005; 44:A3034-A3037.

(263) Ernst E. Iridology: a systematic review. Forsch Komplementärmed 1999; 6:7-9.

(264) White AR, Williamson J, Hart A, Ernst E. A blinded investigation into the accuracy of reflexology. Complement Ther Med 2000; 8:166-172.

(265) Ernst E, Lee MS. Acupressure: an overview of systematic reviews. J Pain Symptom Manage 2010; 40(4):e3-e7.

(266) Kong JC, Lee MS, Shin B-C. Randomized clinical trials on acupuncture in Korean literature: a systematic review. eCAM 2007;doi:10.1093/ecam/nem130.

(267) Ernst E. Acupuncture: what does the most reliable evidence tell us? J Pain Sympt Managem 2009; 37(4):709-714.

(268) Ernst E, Pittler MH, Wider B, Boddy K. Acupuncture: its evidence-base is changing. Am J Chin Med 2007; 35(1):21-25.

(269) Habacher G, Pittler MH, Ernst E. Effectiveness of Acupuncture in Veterinary Medicine: Systematic Review. J Vet Intern Med 2006; 20:480-488.

(270) Ernst E. Acupuncture - a critical analysis. J Intern Med 2006; 259:125-137. 
(271) Ernst E, Canter PH. The Alexander Technique: a systematic review of controlled clinical trials. Forsch Komplementarmed Klass Naturheilkd 2003; 10:325-329.

(272) Vogler BK, Ernst E. Aloe vera: a systematic review of its clinical effectiveness. Br J Gen Pract 1999; 49:823-828.

(273) Ernst E. Anthroposophische Medizin: Geheimwissenschaft oder Heilmethode? Perfusion 2006; 19:344-348.

(274) Cooke B, Ernst E. Aromatherapy: a systematic review. Br J Gen Pract 2000; 50:493-496.

(275) Ernst E, Lee MS. Ayurvedic medicine : an overview of systematic reviews. Perfusion 2010; 23:168-170.

(276) Ernst E. Bach flower remedies: a systematic review of randomised clinical trials. Swiss Med Wkly 2010; 140:w13079.

(277) Ernst E. Systematic reviews of biofeedback. Phys Med Rehab Kuror 2003; 13:321-324.

(278) Ernst E. Chiropractic: a critical evaluation. J Pain Sympt Man 2008; 35(5):544-562.

(279) Ernst E. The "Activator": a systematic review of randomised clinical trials. Perfusion 2009; 22:51-53.

(280) Ernst E. Chiropractic maintenance treatment, a useful preventative approach? Perfusion 2009; 22(5):164-166.

(281) Ernst E, Posadzki P. An independent review of NCCAM-funded studies of chiropractic. Clin Rheumatol 2011.

(282) Ernst E. Colonic irrigation: therapeutic claims by professional organisations, a review. Int J Clin Pract 2010; 64(4):429-431.

(283) Astin JA, Harkness EF, Ernst E. The efficacy of "Distant Healing": a systematic review of randomized trials. Ann Intern Med 2000; 132:903-910.

(284) Ernst E. Distant healing - an "update" of a systematic review. Wien Klin Wochenschr $2003 ; 115: 241-245$.

(285) Harkness EF, Abbot NC, Ernst E. A randomized trial of distant healing for skin warts. Am J Med 2000; 108:448-452.

(286) Ernst E, Canter PH. The Feldenkrais method - a systematic review of randomised clinical trials. Phys Med Rehab Kuror 2005; 15:151-156.

(287) Ernst E. "Flower remedies": a systematic review of the clinical evidence. Wien Klin Wochenschr 2002; 114:963-966.

(288) Ernst E. Frankincense: systematic review. BMJ 2008; 337:a2813.

(289) Borrelli F, Izzo AA, Ernst E. Pharmacological effects of Cimicifuga racemosa. Life Sci 2003; 73:1215-1229. 
(290) Pittler MH, Ernst E. Clinical effectiveness of garlic (Allium sativum). Mol Nutr Food Res 2007; 51:1382-1385.

(291) Chrubasik S, Pittler MH, Roufogalis BD. Zingiberis rhizoma: A comprehensive review on the ginger effect and efficacy profiles. Phytomedicine 2005; 12:684-701.

(292) Vogler BK, Pittler MH, Ernst E. The efficacy of ginseng. A systematic review of randomized clinical trials. Eur J Clin Pharmacol 1999; 55:567-575.

(293) Ernst E. Panax ginseng: an overview of the clinical evidence. J Ginseng Res 2010; 34(4):259-263.

(294) Ernst E. The risk-benefit profile of commonly used herbal therapies: ginkgo, St. John's wort, ginseng, echinacea, saw palmetto and kava. Ann Intern Med 2002; 136:42-53.

(295) Guo R, Canter PH, Ernst E. A systematic review of randomised clinical trials of individualised herbal medicine in any indication. Postgrad Med J 2007; 83:633-637.

(296) Ernst E. Classical homoeopathy versus conventional treatments: a systematic review. Perfusion 1999; 12:13-15.

(297) Ernst E, Pittler MH. Efficacy of homeopathic arnica. A systematic review of placebocontrolled clinical trials. Arch Surg 1998; 133:1187-1190.

(298) Ernst E. Are highly dilute homoeopathic remedies placebos? Perfusion 1998; 11:291-292.

(299) Ernst E. A systematic review of systematic reviews of homeopathy. Br J Clin Pharmacol $2002 ; 54: 577-582$.

(300) Altnuc U, Pittler MH, Ernst E. Homeopathy for Childhood and Adolescence Ailments: Systematic Review of Randomized Clinical Trials. Mayo Clin Proc 2007; 82(1):69-75.

(301) Ernst E. Homeopathy: what does the "best" evidence tell us? Med J Aust 2010; 192(8):458-460.

(302) Ernst E, Pittler MH, Boddy K. Homeopathy: is the evidence-base changing? Perfusion 2006; 19(12):380-382.

(303) Ernst E, Schmidt K. Homotoxicology - a review of randomised clinical trials. Eur J Clin Pharmacol 2004; 60:299-306.

(304) Ernst E. Kombucha: a systematic review of the clinical evidence. Forsch Komplementär Klass Naturheilkd 2003; 10:85-87.

(305) Ernst E, Fialka V. The clinical effectiveness of massage therapy - a critical review. Forsch Komplementarmedizin 1994; 1:226-231.

(306) Ernst E, Pittler MH, Wider B, Boddy K. Massage therapy: Is its evidence-base getting stronger. Comp Health Pract Rev 2007; 12(3):179-183.

(307) Ernst E, Pittler MH, Wider B. Mind-body therapies: are the trial data getting stronger? Altern Ther Health Med 2007; 13(5):62-64. 
(308) Lee MS, Kang JW, Ernst E. Does moxibustion work? An overview of systematic reviews. BMC Res Notes 2010; 3:284.

(309) Ernst E, Fialka V. Die Neuraltherapie im Licht neurer Daten. Fortschr Med 1994; 112:433434.

(310) Ernst E, Köder K. An overview of reflexology. Eur J Gen Pract 1997; 3:52-57.

(311) Ernst E. Is reflexology an effective intervention? A systematic review of randomised controlled trials. Med J Aust 2009; 191(5):263-266.

(312) Ernst E, Posadzki P, Lee MS. Reflexology: an update of a systematic review of randomised clinical trials. Maturitas 2011; 68(2):116-120.

(313) Lee MS, Pittler MH, Ernst E. Effects of reiki in clinical practice: a systematic review of randomised clinical trials. Int J Clin Pract 2008; 62(6):947-954.

(314) Hung SK, Perry R, Ernst E. The effectiveness and efficacy of Rhodiola rosea L.: A systematic review of randomized clinical trials. Phytomedicine 2010.

(315) Ernst E. Does spinal manipulation have specific treatment effects? Fam Pract 2000; 17(6):554-556.

(316) Ernst E, Harkness EF. Spinal manipulation: a systematic review of sham-controlled, double-blind, randomized clinical trials. J Pain Sympt Man 2001; 24:879-889.

(317) Ernst E, Canter PH. A systematic review of systematic reviews of spinal manipulation. J R Soc Med 2006; 99:192-196.

(318) Ernst E. Chiropractic spinal manipulation for the elderly? In: Cherniack P, Cherniack N (eds). Alternative medicine for the elderly. Berlin, Heidelberg; Springer-Verlag. 2003;145152.

(319) Canter PH, Ernst E. Sources of bias in reviews of spinal manipulation for back pain. Wien Klin Wochenschr 2005; 117:333-341.

(320) Ernst E, Huntley A. Tea tree oil: a systematic review of randomised clinical trials. Forsch Komplementärmed 2000; 114:27-29.

(321) Blasi ZD, Harkness E, Ernst E, Georgiou A, Kleijnen J. Influence of context effects on health outcomes: a systematic review. Lancet 2001; 357:757-762.

(322) Ernst E, Lee MS. How effective is yoga? A concise overview of systematic reviews. FACT 2010; 15(4):274-279.

(323) Hung SK, Hillier S, Ernst E. Case reports of adverse effects of herbal medicinal products (HMPs): A quality assessment. Phytomedicine 2010.

(324) Ernst E. Harmless herbs? A review of the recent literature. Am J Med 1998; 104:170-178.

(325) Ernst E. Risks associated with complementary therapies. In: Dukes MNG, Aronson JK (eds). Meyler's Side Effects of Drugs. 14th ed. Amsterdam: Elsevier. 2000;1649-1681. 
(326) Ernst E, Pittler MH. Risks associated with herbal medicinal products. Wien Med Wschr 2002; 152:183-189.

(327) Ernst E. Treatments used in complementary and alternative medicine. Ch48. In Aronson, J.K. (ed). Side Effects of Drugs, Annual 25. Amsterdam; Elsevier science B.V.. 2002;567578.

(328) Ernst E, Canter P.H., Thompson Coon J. Does Ginkgo biloba increase the risk of bleeding? A systematic review of case reports. Perfusion 2005; 18:52-56.

(329) Pittler MH, Ernst E. Systematic review: hepatotoxic events associated with herbal medicinal products. Ailment Pharmacol Ther 2003; 18:451-471.

(330) Pittler MH, Schmidt K, Ernst E. Adverse events of herbal food supplements for body weight reduction: systematic review. Obesity Rev 2005; 6:93-111.

(331) Ernst E. Cardiovascular adverse effects of herbal medicines: a systematic review of the recent literature. Can J Cardiol 2003; 19:818-827.

(332) Ernst E. Serious psychiatric and neurological adverse effects of herbal medicines - a systematic review. Acta Psychiatr Scand 2003; 108:83-91.

(333) Daniele C, Thompson Coon J, Pittler MH, Ernst E. Vitex agnus castus. A systematic review of adverse events. Drug Saf 2005; 28:319-332.

(334) Daniele C, Mazzanti G, Pittler MH, Ernst E. Adverse-event profile of Crataegus spp.: a systematic review. Drug Saf 2006; 29:523-535.

(335) Huntley AL, Thompson Coon J, Ernst E. The safety of herbal medicinal products derived from Echinacea species. A systematic review. Drug Saf 2005; 28:387-400.

(336) Thompson Coon J, Ernst E. Panax ginseng. A systematic review of adverse effects and drug interactions. Drug Saf 2002; 25:323-344.

(337) Stevinson C, Huntley A, Ernst E. A systematic review of the safety of kava extract in the treatment of anxiety. Drug Saf 2002; 25(4):251-261.

(338) Ernst E. Nutzen unter-, Risiken überschä. MMW Fortschr Med 2003; 41:40.

(339) Ernst E. Safety concerns about kava. Lancet 2002; 359:1865.

(340) UK BEAM Trial Team. United Kingdom back pain exercise and manipulation (UK BEAM) randomised trial: effectiveness of physical treatments for back pain in primary care. BMJ, doi:10.1136/bmj.38282.669225.AE. BMJ 2004; 19 November:1-8.

(341) Huntley A, Ernst E. A systematic review of the safety of black cohosh. Menopause 2003; 10:58-64.

(342) Agbabiaka TB, Pittler MH, Wider B, Ernst.E. Serenoa repens (saw palmetto): a systematic review of adverse events. Drug Saf 2009; 32(8):637-647.

(343) Ernst E. Herbal medicinal products during pregnancy: are they safe? BJOG: an Int J Obstet Gynae 2002; 109:227-235. 
(344) Ernst E. Serious adverse effects of unconventional therapies for children and adolescents: a systematic review of recent evidence. Eur J Pediatr 2003; 162:72-80.

(345) Ernst E. Adverse effects of herbal drugs in dermatology. Br J Dermatol 2000; 143:923929.

(346) Gundling K, Ernst E. Herbal medicines: influences on blood coagulation. Perfusion 2001; 14:336-342.

(347) Smith L, Ernst E, Ewings P, Allen J, Smith C, Quinlan C. What affects anticoagulation control in patients taking warfarin? Br J Gen Pract 2009; 59:590-594.

(348) Ernst E. Possible interactions between synthetic and herbal medicinal products. Part 1: a systematic review of the indirect evidence. Perfusion 2000; 13:4-6,8.

(349) Ernst E. Interactions between synthetic and herbal medicinal products. Part 2: a systematic review of the direct evidence. Perfusion 2000; 13:60-70.

(350) Ernst E. Herb-drug interactions - an update. Perfusion 2003; 16:175-194.

(351) Ernst E. St John's wort supplements endanger the success of organ transplantation. Arch Surg 2002; 137:316-319.

(352) Ernst E. Herb-drug interactions: potentially important but woefully under-researched. Eur J Clin Pharmacol 2000; 56:523-524.

(353) Smith L, Ernst E, Ewings P, Myers P, Smith C. Co-ingestion of herbal medicines a warfarin. Br J Gen Pract 2004; 54:439-441.

(354) Izzo AA, Ernst E. Interactions between herbal medicines and prescribed drugs: a systematic review. Drugs 2001; 15:2163-2175.

(355) Thompson Coon J, Pittler M, Ernst E. Herb-drug interactions: survey of leading pharmaceutical/herbal companies. Arch InternMed 2003; 163:1371.

(356) Izzo AA, Di Carlo G, Borrelli F, Ernst E. Cardiovascular pharmacotherapy and herbal medicines: the risk of drug interaction. Int J Cardiology 2005; 98:1-14.

(357) Huntley A. Drug-herb interactions with herbal medicines for menopause. J Br Menopause Soc 2004; Dec:162-165.

(358) Izzo AA, Ernst E. Interactions Between Herbal Medicines and Prescribed Drugs: An Updated Systematic Review. Drugs 2009; 69(13):1777-1798.

(359) Savovic J, Wider B, Ernst E. Effects of Ginkgo biloba on blood coagulation parameters: a systematic review of randomised controlled trials. Evidence-Based Integrative 2005; $2: 167-176$

(360) Ernst E. Heavy metals in traditional Indian remedies. Eur J Clin Pharmacol 2001; 57:891896.

(361) Ernst E, Thompson Coon J. Heavy metals in traditional Chinese medicines: a systematic review. Clin Pharmacol Ther 2001; 70:497-504. 
(362) Ernst E. Adulteration of Chinese herbal medicines with synthetic drugs: a systematic review. J Internal Med 2002; 252:107-113.

(363) Ernst E, Sieder C, März R. Adverse drug reactions to herbal and synthetic expectorants. Int J Risk Safety Med 1995; 7:219-225.

(364) Ernst E, Rand JI, Barnes J, Stevinson C. Adverse effects profile of the herbal antidepressant St. John's wort (Hypericum perforatum L.). Eur J Clin Pharmacol 1998; 54:589-594.

(365) Stevinson C, Ernst E. Safety of Hypericum in patients with depression: a comparison with conventional antidepressants. CNS Drugs 1999; 11:125-132.

(366) Stevinson C, Ernst E. Can St. John's wort trigger psychoses? Int J Clin Pharmacol and Ther 2004; 42:473-480.

(367) Ernst E, White A. Life-threatening adverse reactions after acupuncture? A systematic review. Pain 1997; 71:123-126.

(368) Yamashita H, Tsukayama H, White AR, Tanno Y, Sugishita C, Ernst E. Systematic review of adverse events following acupuncture: the Japanese literature. Complementar Ther Med 2001; 9:98-104.

(369) Peuker ET, White A, Ernst E, Pera F, Filler TJ. Traumatic Complications of Acupuncture. Arch Fam Med 1999; 8:553-557.

(370) Ernst E, White AR. Prospective studies of the safety of acupuncture: a systematic review. Am J Med 2001; 110:481-485.

(371) White A, Hayhoe S, Hart A, Ernst E. Adverse events following acupuncture: prospective survey of 32000 consultations with doctors and physiotherapists. BMJ 2001; 323:485-486.

(372) White AR, Hayhoe S, Ernst E. Survey of adverse events following acupuncture. Acupunct Med 1997; 15:67-70.

(373) Ernst E, Sherman KJ. Is acupuncture a risk factor for hepatitis? Systematic review of epidemiological studies. J Gastroenterol Hepatol 2003; 18:1231-1236.

(374) Ernst E, Zhang J. Cardiac tamponade caused by acupuncture: A review of the literature. Int J Cardiol 2010.

(375) Zhang J, Shang H, Gao X, Ernst E. Acupuncture-related adverse events: a systematic review of the Chinese literature. Bull World Health Organ 2010; 88(12):915-921C.

(376) Ernst E. Deaths after acupuncture: a systematic review. Int J Risk \& Safety 2010; 22:1-6.

(377) Ernst E. Serious adverse effects after acupuncture - a systematic review (2000-2009). Perfusion 2010; 23(6):214-225.

(378) Ernst E. Deaths associated with EDTA chelation therapy - a systematic review. Perfusion 2009; 22(1):9-11.

(379) Stevinson C, Honan W, Cooke B, Ernst E. Neurological complications of cervical spine manipulation. J Roy Soc Med 2001; 94:107-110. 
(380) Ernst E. Life-threatening complications of spinal manipulation. Stroke 2001; 32:809-810.

(381) Ernst E. Prospective investigations into the safety of spinal manipulation. J Pain Sympt Managem 2001; 21:238-242.

(382) Stevinson C, Ernst E. Risks associated with spinal manipulation. Am J Med 2002; 112:566-570.

(383) Ernst E. Cerebrovascular complications associated with spinal manipulation. Phys Ther Rev 2004; 9:5-15.

(384) Ernst E. Manipulation of the cervical spine: a systematic review of case reports of serious adverse events, 1995-2001. MJA 2002; 176:376-380.

(385) Ernst E. Chiropractors' use of X-rays. Br J Radiol 1998; 71:249-251.

(386) Ernst E. Attitude against immunisation within some branches of complementary medicine. Eur J Pediatr 1997; 156:513-515.

(387) Ernst E. Chiropractic care: attempting a risk-benefit analysis. Am J Public Health 2002; 92:1603-1604.

(388) Ernst E. Ophthalmological adverse effects of (chiropractic) upper spinal manipulation: evidence from recent case reports. Acta Ophthalmol Scand 2005; 83:581-585.

(389) Ernst E. Adverse effects of spinal manipulation: a systematic review. J R Soc Med 2007; 100(7):330-338.

(390) Shin BC, Lee MS, Park T-Y, Ernst E. Serious adverse events after spinal manipulation: a systematic review of the Korean literature. FACT 2010; 15(3):198-201.

(391) Ernst E. Deaths after chiropractic: a review of published cases. Int J Clin Pract 2010; 64(8):1162-1165.

(392) Ernst E. The safety of massage therapy. Rheumatology (Oxford) 2003; 42:1101-1106.

(393) Park JE, Lee SS, Lee MS, Choi SM, Ernst E. Adverse events of moxibustion: a systematic review. Complement Ther Med 2010; 18(5):215-223.

(394) Ernst E, Schmidt K. Health risks over the internet: advice offered by "medical herbalists" to a pregnant woman. Wien Med Wschr 2002; 152:190-192.

(395) Schmidt K, Ernst E. Internet advice by acupuncturists - a risk factor for cardiovascular patients? Perfusion 2002; 15:44-50.

(396) Ernst E. Rise in popularity of complementary and alternative medicine: reasons and consequences for vaccination. Vaccine 2002; 20:S90-S93.

(397) Ernst E. Complementary medicine: its impact on vaccination. Vaccines 2002; 5:46-48.

(398) Schmidt K, Ernst E. Aspects of MMR. BMJ 2002; 325:597.

(399) Schmidt K, Ernst E. Letter to the Editor: Are asthma sufferers at risk when consulting chiropractors over the Internet? Respiratory Med 2003; 97:104-105. 
(400) Ernst E, Gilbey A. Chiropractic claims in the English-speaking world. New Zealand Med J 2010; 123(1312):36-44.

(401) Ernst E. Advice issued by professional homeopathic associations: a survey of websites. $\mathrm{Br}$ J Gen Pract 2009; 59(559):142-143.

(402) Ernst E. The ethics of British professional homoeopaths. Int J Clin Pract 2010; 64(2):147148.

(403) Schmidt K, White A, Ernst E. Reflexologists' responses to a patient with abdominal pain a survey on Internet advice. Complementar Ther Med 2003; 11:98-102.

(404) Schmidt K, Ernst E. Complementary/alternative medicine for diabetes. How good is advice offered on websites? Diabetic Med 2003; 20:247-251.

(405) Ernst E, Schmidt K. 'Alternative' cancer cures via the Internet? Br J Cancer 2002; 87:479480.

(406) Schmidt K, Ernst E. Assessing websites on complementary and alternative medicine for cancer. Ann Oncol 2004; 15:733-742.

(407) Gunasekera V, Ernst E, Ezra DG. Systematic internet-based review of complementary and alternative medicine for glaucoma. Ophthalmology 2008; 115(3):435-439.

(408) White AR, Ernst E. Economic analysis of complementary medicine: a systematic review. Complement Ther Med 2000; 8:111-118.

(409) Kernick D, White AR. Applying economic evaluation to complementary and alternative medicine. In: Getting Health Economics into Practice. Kernick, D.E. (ed). Radcliffe Medical Press, Oxford. 2002;173-179.

(410) Canter PH, Thompson Coon J, Ernst E. Cost effectiveness of complementary treatments in the United Kingdom: systematic review. BMJ 2005; 331(880):881.

(411) Canter P.H., Thompson Coon J, Ernst E. Cost-Effectiveness of Complementary Therapies in the United Kingdom-A Systematic Review. eCAM Advance Access 2006; doi:10.1093/ecam/nel044.

(412) Thompson Coon J, Ernst E. A systematic review of the economic evaluation of complementary and alternative medicine. Perfusion 2005; 18:202-214.

(413) Ernst E. The role of complementary and alternative medicine. BMJ 2000; 321:1133-1135.

(414) Ernst E, Pittler MH, Stevinson C, White AR. The Desktop Guide to Complementary and Alternative Medicine. 1st Edition. Edinburgh: Mosby. 2001.

(415) Ernst E, Pittler MH, Wider B, Boddy K. The Desktop Guide to Complementary and Alternative Medicine. 2nd edition. Edinburgh: Elsevier Mosby. 2006. 